\title{
Synthesis of unsymmetrical 1,4-dicarbonyl compounds by photocatalytic oxidative radical additions
}

\author{
Ya Dong, Ruining Li, Junliang Zhou and Zhankui Sun* \\ Shanghai Key Laboratory for Molecular Engineering of Chiral Drugs, Pharm-X Center, \\ School of Pharmacy, Shanghai Jiao Tong University, No. 800 Dongchuan Rd., Shanghai \\ 200240, China. \\ $\square_{\text {email: zksun@sjtu.edu.cn }}$
}




\section{Table of Contents}

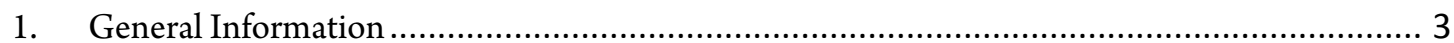

2. Reaction Optimization and Control Experiments........................................................... 4

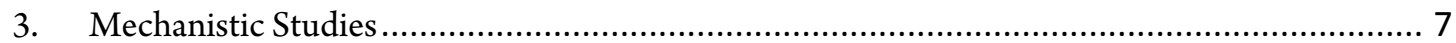

4. General Procedures and Experimental Data .................................................................... 16

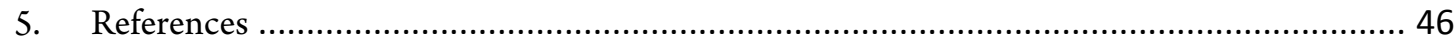

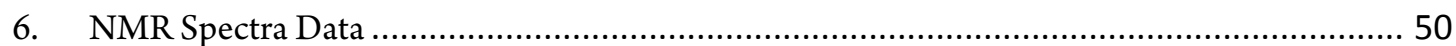




\section{General Information}

Commercial chemicals were used as supplied without further purification. The solvents were used directly from commercial reagents. The iridium photocatalysts $\operatorname{Ir}\left(\mathrm{dF}(\mathrm{CF} 3)\right.$ ppy2(dtbbpy)PF6 (Ir-1), $\left[\operatorname{Ir}\left(\mathrm{dF}\left(\mathrm{CF}_{3}\right) \text { ppy }\right)_{2}(\mathrm{dOMebpy})\right]--\mathrm{PF}_{6}$ (Ir-2), and (fac)-Ir(ppy $)_{3}(\mathbf{I r}-3)$ were synthesized following the published route ${ }^{1}$. Organic solutions were concentrated under reduced pressure on an IKA rotary evaporator using a water bath. Chromatographic purification of products was accomplished using force-flow chromatography on silica gel (200-300 mesh) according to the method of Still ${ }^{2}$. Thin-layer chromatography (TLC) was performed on $250 \mu \mathrm{m}$ silica gel plates. TLC visualization was performed by UV lamp and $\mathrm{KMnO}_{4}$ stain $\left(1 \mathrm{~g} \mathrm{KMnO}, 1 \mathrm{~g} \mathrm{NaHCO}\right.$ and $\left.100 \mathrm{~mL} \mathrm{H}_{2} \mathrm{O}\right)$. All key compounds were characterized by ${ }^{1} \mathrm{H}$ NMR, ${ }^{13} \mathrm{C}$ NMR and HRMS. ${ }^{1} \mathrm{H}$ NMR spectra were recorded on an Agilent NMR Systems $400 \mathrm{MHz}$ Spectrometer $\left({ }^{1} \mathrm{H}\right.$ NMR at $\left.400 \mathrm{MHz}\right)$ and are internally referenced to residual protic $\mathrm{CDCl}_{3}(\delta 7.26 \mathrm{ppm})$ unless otherwise noted. Data for ${ }^{1} \mathrm{H}$ NMR are reported as follows: chemical shift $(\delta \mathrm{ppm})$, multiplicity $(\mathrm{s}=$ singlet, $\mathrm{d}$ $=$ doublet, $\mathrm{t}=$ triplet, $\mathrm{q}=$ quartet, $\mathrm{m}=$ multiplet, $\mathrm{dd}=$ doublet of doublets, $\mathrm{dt}=$ doublet of triplets, brs = broad singlet), and coupling constant $(\mathrm{Hz}) \cdot{ }^{13} \mathrm{C}$ NMR spectra were recorded on an Agilent NMR Systems $400 \mathrm{MHz}$ Spectrometer $\left({ }^{13} \mathrm{C} \mathrm{NMR}\right.$ at $\left.101 \mathrm{MHz}\right)$ and data are reported in terms of chemical shift relative to $\mathrm{CDCl}_{3}$ (77.16 ppm). For ${ }^{1} \mathrm{H}$ NMR yield analysis of crude reaction mixtures, mesitylene was used as internal standard, and added as pure liquid. HRMS: electrospray ionization mass (ESI-MS) was performed on Agilent 6210 Series TOF MS. The blue LEDs were purchased from supermarket and directly used without any filters. The brand name of LEDs is Jin Dian Yuan and the model number is JDY-TG01. The shell material Seiko aluminum + PC. 


\section{Reaction Optimization and Control Experiments}

General procedure: To a $25 \mathrm{~mL}$ round bottomed flask equipped with a magnetic stirrer bar were added (1-bromovinyl) benzene ( $91 \mathrm{mg}, 0.5 \mathrm{mmol}, 1.0$ equiv.), ethyl 2-mercaptoacetate (90 mg, $0.75 \mathrm{mmol}, 1.5$ equiv.), photocatalyst, phosphoric reagent, and solvent $(7 \mathrm{~mL}$ ). The flask was quickly degassed three times and flushed with oxygen balloon, and then the mixture was irradiated with three $45 \mathrm{~W}$ blue LEDs ( $5 \mathrm{~cm}$ away) at room temperature for 20 hours. The reaction mixture was filtered and concentrated. The residue was purified by column chromatography on silica with petroleum ether/ ethyl acetate as eluent. 
Table S1 Optimization of reaction conditions

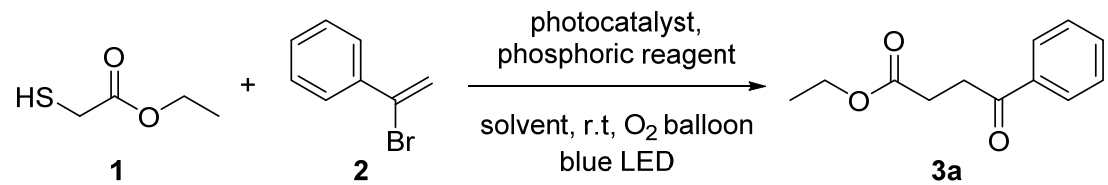

\begin{tabular}{|c|c|c|c|c|c|}
\hline Entry & $\mathrm{PR}_{3}$ & photocatalyst & solvent & Time & yields $^{c}$ \\
\hline $1^{a}$ & $\mathrm{PPh}_{3} 5$ eq & DTBP 4 eq & DCM & $20 \mathrm{~h}$ & $38 \%$ \\
\hline 2 & $\mathrm{PPh}_{3} 5$ eq & 4CzIPN 5 mol\% & DCM & $20 \mathrm{~h}$ & $10 \%$ \\
\hline $3^{a}$ & $\mathrm{PPh}_{3} 5 \mathrm{eq}$ & $\mathrm{Ru}(\mathrm{bpy})_{3} \mathrm{Cl}_{2} 5 \mathrm{~mol} \%$ & $\mathrm{DCM}$ & $20 \mathrm{~h}$ & $1 \%$ \\
\hline 4 & $\mathrm{PPh}_{3} 5$ eq & Mes-Acr-Me 5 mol\% & $\mathrm{DCM}$ & $20 \mathrm{~h}$ & $17 \%$ \\
\hline 5 & $\mathrm{PPh}_{3} 5$ eq & Ir-1 2 mol\% & $\mathrm{DCM}$ & $20 \mathrm{~h}$ & $50 \%$ \\
\hline 6 & $\mathrm{PPh}_{3} 5$ eq & Ir-2 2 mol\% & DCM & $48 \mathrm{~h}$ & $34 \%$ \\
\hline 7 & $\mathrm{PPh}_{3} 5$ eq & Ir-3 2 mol\% & DCM & $48 \mathrm{~h}$ & $64 \%$ \\
\hline 8 & $\mathrm{PPh}_{3} 5$ eq & Ir-1 2 mol\% & EA & $20 \mathrm{~h}$ & $<1 \%$ \\
\hline 9 & $\mathrm{PPh}_{3} 5$ eq & Ir-1 2 mol\% & $\mathrm{MeCN}$ & $20 \mathrm{~h}$ & $<1 \%$ \\
\hline 10 & $\mathrm{PPh}_{3} 5$ eq & Ir-1 2 mol\% & $\mathrm{MeOH}$ & $20 \mathrm{~h}$ & $<1 \%$ \\
\hline 11 & $\mathrm{PPh}_{3} 5$ eq & Ir-1 2 mol\% & $\mathrm{CHCl}_{3}$ & $20 \mathrm{~h}$ & $<1 \%$ \\
\hline 12 & $\mathrm{PPh}_{3} 5$ eq & Ir-1 2 mol\% & DMF & $20 \mathrm{~h}$ & $<1 \%$ \\
\hline 13 & $\mathrm{PPh}_{3} 5$ eq & Ir-1 2 mol\% & DMSO & $20 \mathrm{~h}$ & $<1 \%$ \\
\hline 14 & $\mathrm{PPh}_{3} 5$ eq & Ir-1 2 mol\% & DCE & $20 \mathrm{~h}$ & $33 \%$ \\
\hline 15 & $\mathrm{P}(\mathrm{OEt})_{3} 5 \mathrm{eq}$ & Ir-1 2 mol\% & $\mathrm{DCM}$ & $20 \mathrm{~h}$ & $13 \%$ \\
\hline 16 & $\mathrm{PPh}_{2} \mathrm{OEt} 5$ eq & Ir-1 2 mol\% & $\mathrm{DCM}$ & $20 \mathrm{~h}$ & $7 \%$ \\
\hline $17^{b}$ & $\mathrm{PPh}_{3} 3.5 \mathrm{eq}$ & Ir-1 2 mol\% & $\mathrm{DCM}$ & $7 \mathrm{~h}$ & $94 \%$ \\
\hline 18 & $\mathrm{PPh}_{3} 1.5 \mathrm{eq}$ & Ir-1 2 mol\% & DCM & $7 \mathrm{~h}$ & $25 \%$ \\
\hline
\end{tabular}

${ }^{a} \mathrm{hv}$ as the light resource, ${ }^{b} \mathrm{TEA}(1 \mathrm{eq})$ was addictive 
Table S2 Control experiments

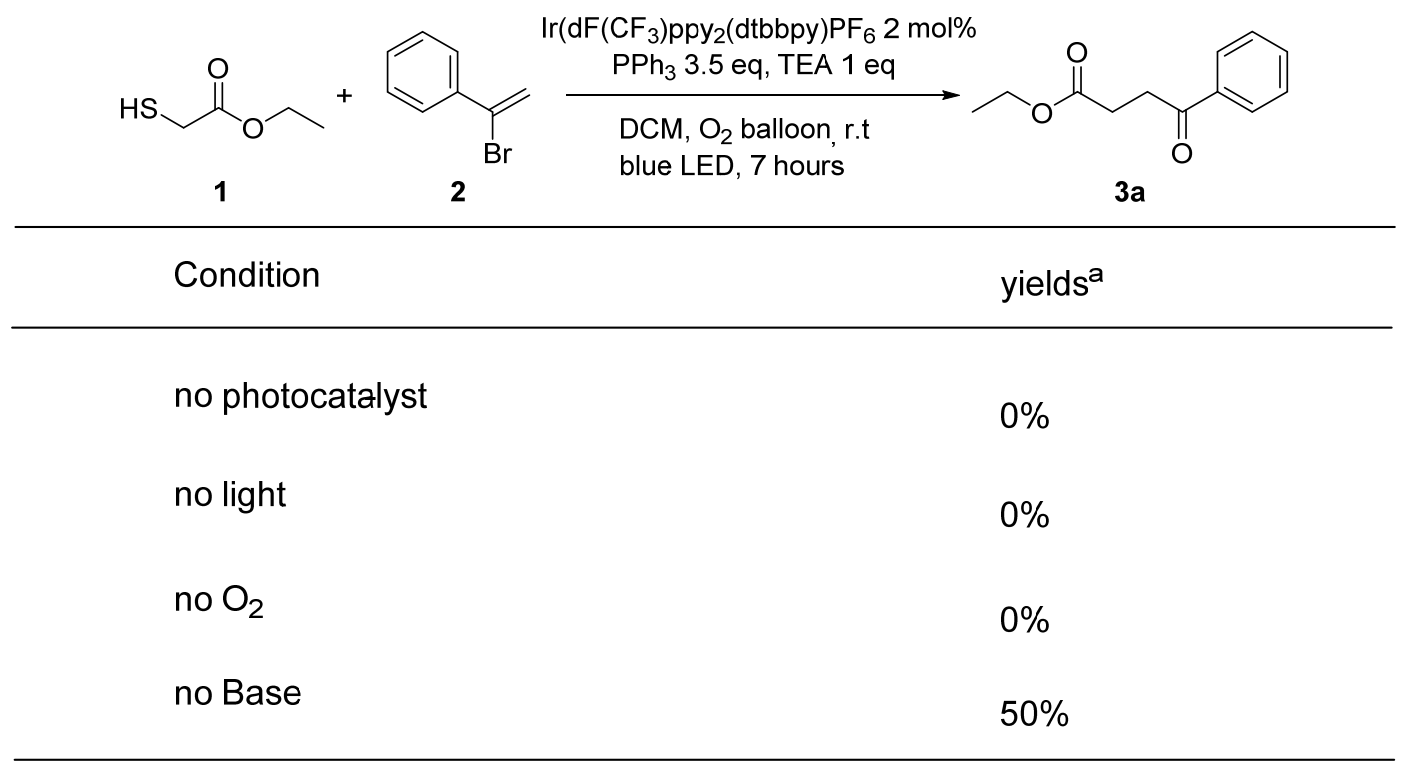

ayields determined by ${ }^{1} \mathrm{H}$ NMR analysis 


\section{Mechanistic Studies}

(a)

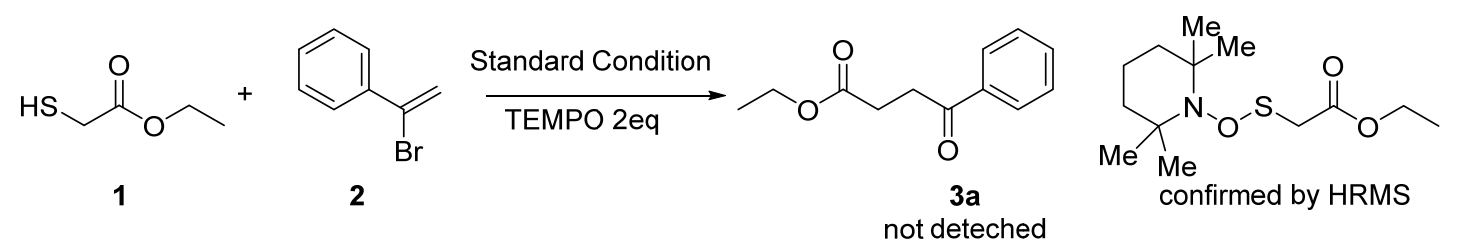

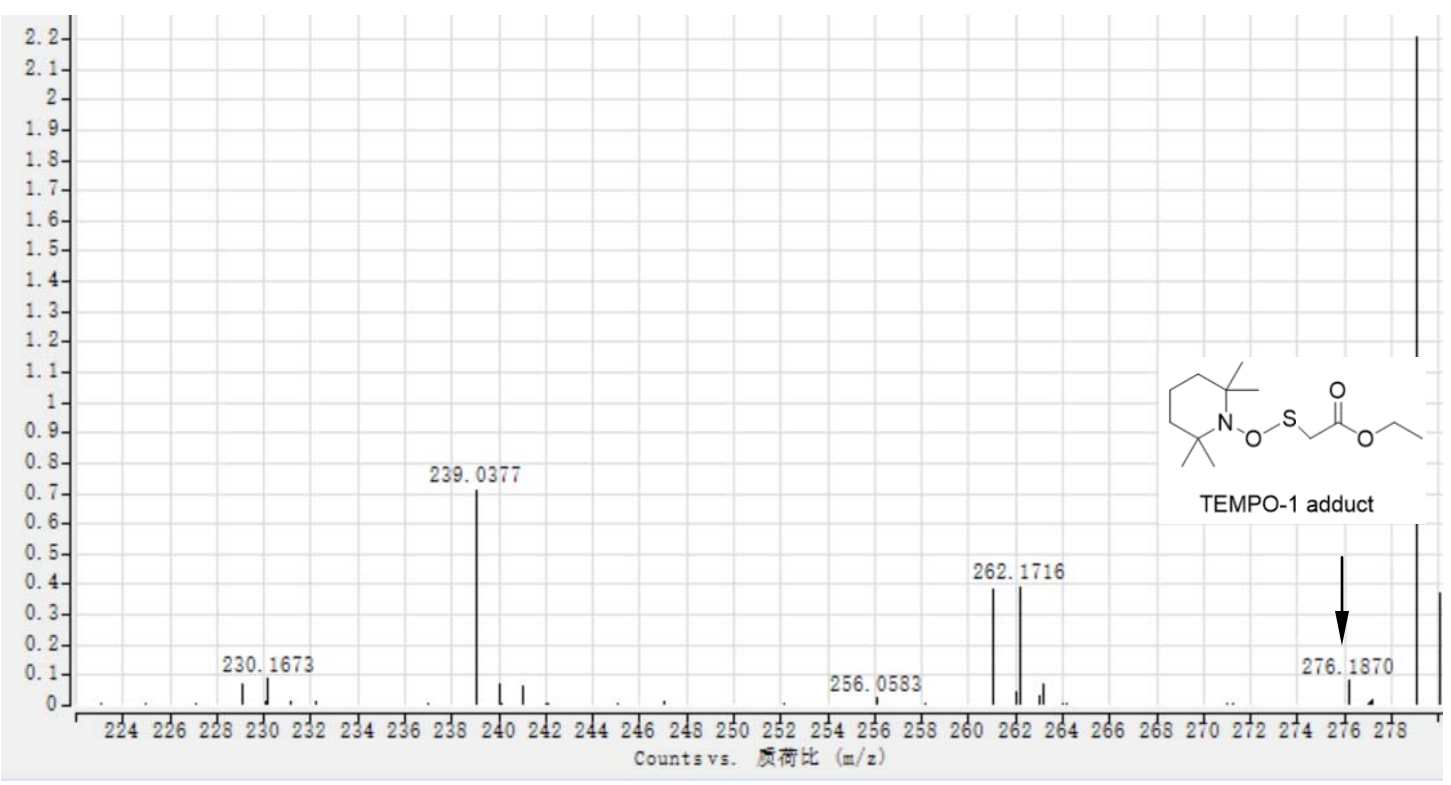

Figure S1

Radical-inhibitor experiment was executed as follows: to a $25 \mathrm{~mL}$ round-bottom flask equipped with a stir bar was added (1-bromovinyl) benzene (91 mg, $0.5 \mathrm{mmol}, 1.0$ equiv.), ethyl 2-mercaptoacetate ( $90 \mathrm{mg}, 0.75 \mathrm{mmol}, 1.5$ equiv.), $\operatorname{Ir}\left(\mathrm{dF}\left(\mathrm{CF}_{3}\right) \mathrm{ppy}_{2}(\mathrm{dtbbpy}) \mathrm{PF}_{6}(10\right.$ $\mathrm{mg}, 0.01 \mathrm{mmol}, 0.02$ equiv.), $\mathrm{PPh}_{3}(458 \mathrm{mg}, 1.75 \mathrm{mmol}, 3.5$ equiv.), triethylamine (50 mg, $0.5 \mathrm{mmol}, 1.0$ equiv.), TEMPO (2.0 equiv.) and DCM $(7 \mathrm{~mL})$. The flask was quickly degassed three times and flushed with oxygen through balloon. Then the mixture was stirred at room temperature and irradiated with three $45 \mathrm{~W}$ blue LEDs ( $5 \mathrm{~cm}$ away) for 7 hours. Eventually, the reaction was completely inhibited. TEMPO-1 adduct was observed in the LC-MS while there was no 3a (Figure S1). 
$(\mathbf{b})$
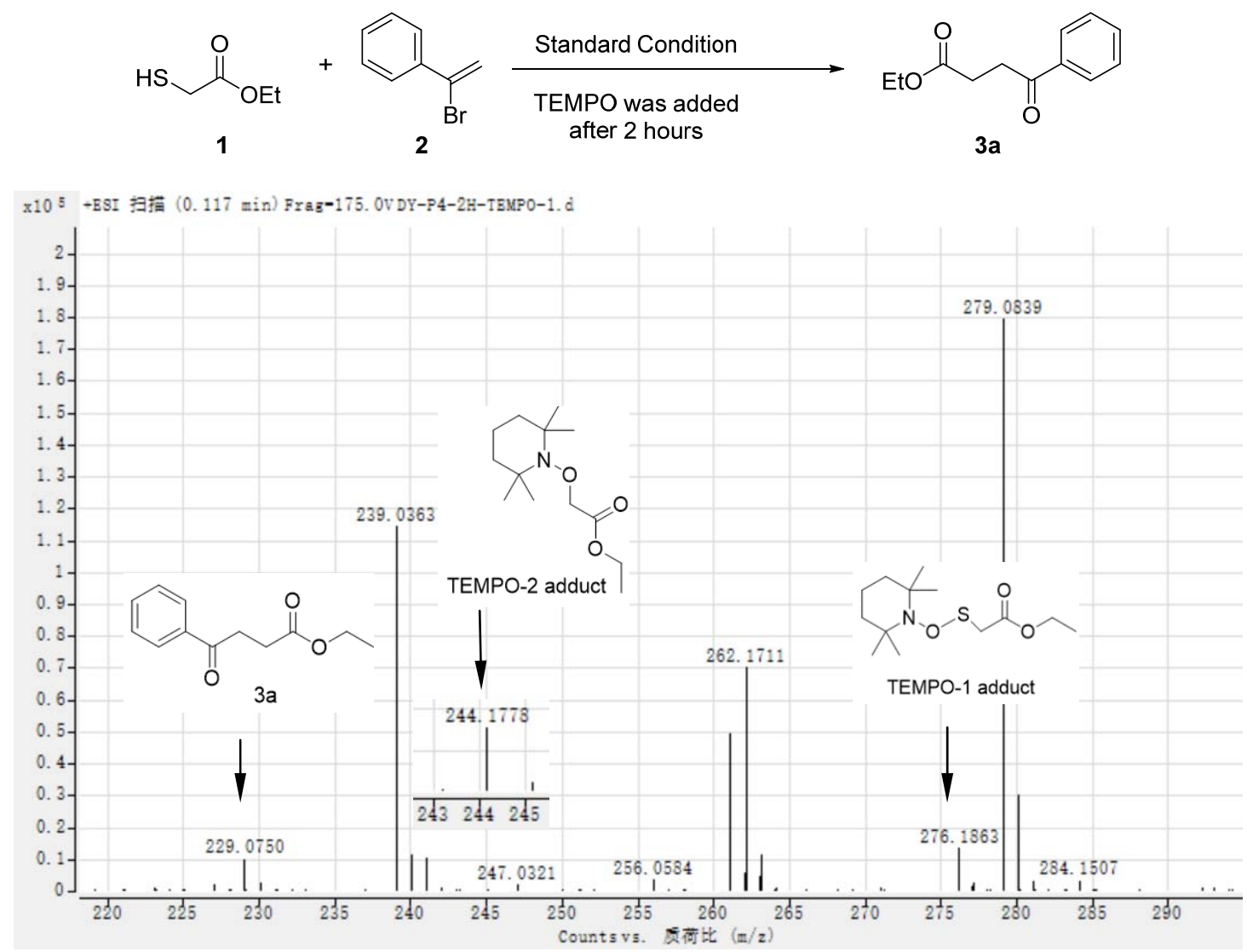

Figure S2

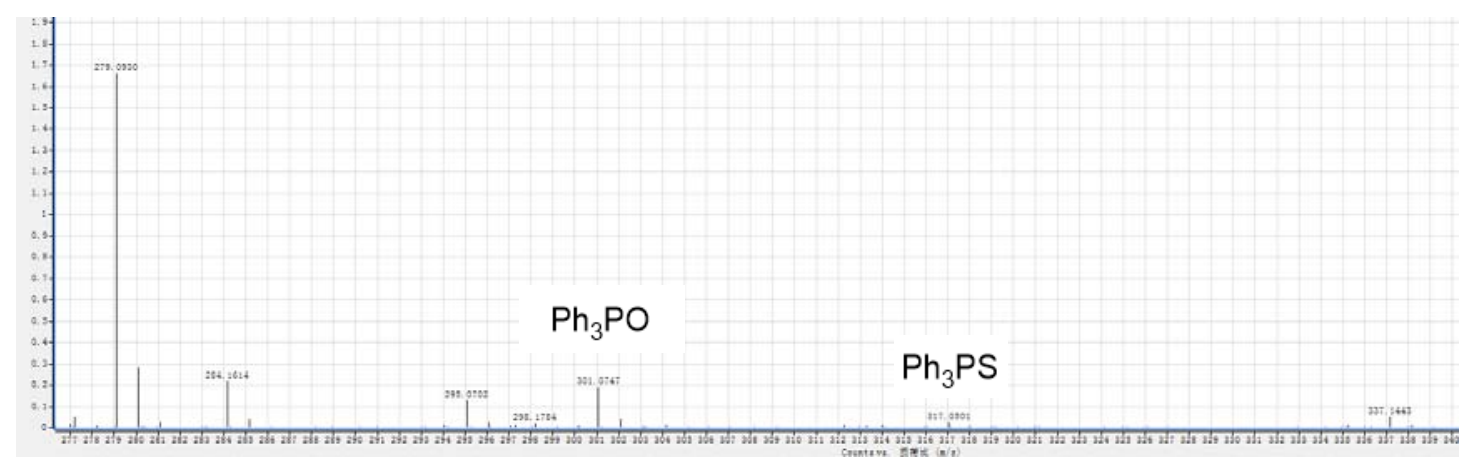

Figure S3

To further investigate the mechanism, another radical-inhibitor experiments was executed as follows: to a $25 \mathrm{~mL}$ round-bottom flask equipped with a stir bar was added (1-bromovinyl)-benzene (91 mg, $0.5 \mathrm{mmol}, 1.0$ equiv.), ethyl 2-mercaptoacetate $(90 \mathrm{mg}$, 0.75 mmol, 1.5 equiv.), $\operatorname{Ir}\left(\mathrm{dF}\left(\mathrm{CF}_{3}\right) \mathrm{ppy}_{2}\right.$ (dtbbpy) $\mathrm{PF}_{6}(10 \mathrm{mg}, 0.01 \mathrm{mmol}, 0.02$ equiv. $), \mathrm{PPh}_{3}$ (458 mg, $1.75 \mathrm{mmol}, 3.5$ equiv.), triethylamine ( $50 \mathrm{mg}, 0.5 \mathrm{mmol}, 1.0$ equiv.) and DCM (7 $\mathrm{mL}$ ). The flask was quickly degassed three times and flushed with oxygen through balloon. Then the mixture was stirred at room temperature and irradiated with three $45 \mathrm{~W}$ blue LEDs 
( $5 \mathrm{~cm}$ away) for 2 hours. Then, TEMPO (2.0 equiv.) was added into the reaction, and the mixture was stirred continuously for 5 hours. TEMPO-1 adduct, TEMPO-2 adduct and 3a were observed in the LC-MS (Figure S2). $\mathrm{Ph}_{3} \mathrm{PO}$ and $\mathrm{Ph}_{3} \mathrm{PS}$ were observed in the LC-MS (Figure S3).

(c): model reaction on $1 \mathrm{mmol}$ scale

To the solution of (1-bromovinyl) benzene (182 $\mathrm{mg}, 1.0 \mathrm{mmol}$ ) and ethyl 2-mercaptoacetate $(180 \mathrm{mg}, 1.5 \mathrm{mmol}, 1.5$ equiv) in $\mathrm{DCM}(10 \mathrm{~mL})$ were added $\operatorname{Ir}\left(\mathrm{dF}\left(\mathrm{CF}_{3}\right) \mathrm{ppy}_{2}\right.$ (dtbbpy) $\mathrm{PF}_{6}(20 \mathrm{mg}, 1 \% \mathrm{mmol}, 0.02$ equiv $), \mathrm{PPh}_{3}(3.5 \mathrm{mmol}, 3.5$ equiv$)$, and triethylamine (TEA, $1.0 \mathrm{mmol}, 1.0$ equiv). The flask was quickly degassed three times and flushed with oxygen through balloon. The mixture was stirred at room temperature and irradiated with three $45 \mathrm{~W}$ blue LEDs ( $5 \mathrm{~cm}$ away) for 7 hours. The reaction progress was monitored by TLC analysis. The solvent was removed in vacuo and the residue was extracted with ethyl acetate for three times. The combined organic layer was dried over anhydrous $\mathrm{Na}_{2} \mathrm{SO}_{4}$, and concentrated in vacuo. The crude product was purified by silica gel chromatography with EA: PE (1:30 - 1:10). The desired product was obtained in 180mg (87\% yield), together with $290 \mathrm{mg} \mathrm{Ph} 3 \mathrm{PS}(1.0 \mathrm{mmol})$ and $610 \mathrm{mg}(2.2 \mathrm{mmol}) \mathrm{mmol} \mathrm{Ph}_{3} \mathrm{PO}$.

(d):

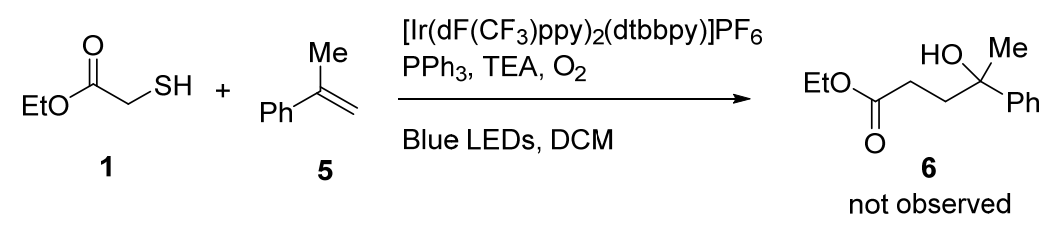

To a $25 \mathrm{~mL}$ round-bottom flask equipped with a stir bar was added prop-1-en-2-ylbenzene (59 mg, $0.5 \mathrm{mmol}, 1.0$ equiv.), ethyl 2-mercaptoacetate (90 mg, $0.75 \mathrm{mmol}, 1.5$ equiv.), $\operatorname{Ir}\left(\mathrm{dF}\left(\mathrm{CF}_{3}\right) \mathrm{ppy}_{2}(\mathrm{dtbbpy}) \mathrm{PF}_{6}(10 \mathrm{mg}, 0.01 \mathrm{mmol}, 0.02\right.$ equiv. $), \mathrm{PPh}_{3}(458 \mathrm{mg}, 1.75 \mathrm{mmol}$, 3.5 equiv.), triethylamine ( $50 \mathrm{mg}, 0.5 \mathrm{mmol}, 1.0$ equiv.) and DCM $(7 \mathrm{~mL})$. The flask was quickly degassed three times and flushed with oxygen through balloon, and then the mixture was stirred at room temperature and irradiated with three $45 \mathrm{~W}$ blue LEDs ( $5 \mathrm{~cm}$ away) for 7 hours. Form LC-MS analysis, we didn't observe product 6. 
(e) Stern-Volmer luminescence quenching experiments

General procedure: To a solution of $\operatorname{Ir}-1\left(\left[\operatorname{Ir}\left\{\mathrm{dF}\left(\mathrm{CF}_{3}\right) \mathrm{ppy}_{2}(\mathrm{dtbbpy})\right] \mathrm{PF}_{6}\right)\right.$ in DCM was added appropriate amount of quencher in a quartz cuvette. Then the emission of the sample was collected. Luminescence spectra was obtained with the detection wavelength of $469 \mathrm{~nm}$ and the luminescence emission spectrum was excited at $425 \mathrm{~nm}$ (excitation maximum of $\left[\operatorname{Ir}\left\{\mathrm{dF}\left(\mathrm{CF}_{3}\right) \mathrm{ppy}_{2}(\mathrm{dtbbpy})\right] \mathrm{PF}_{6}\right)$ using FL6500 (observed in $\left.3^{*} 10^{-5} \mathrm{mM} \mathrm{DCM}\right){ }^{3}$ After the acquisition, the data were ploted according to the Stern - Volmer equation ${ }^{4}$ shown below.

$$
\mathrm{I}_{0} / \mathrm{I}=1+\mathrm{KSV}[\mathrm{Q}]
$$

Where $\mathrm{I}_{0}$ is the luminescence intensity in the absence of the quencher, $\mathrm{I}$ is the intensity in the presence of the quencher, KSV is the Stern-Volmer constant, and [Q] is the concentration of the quencher

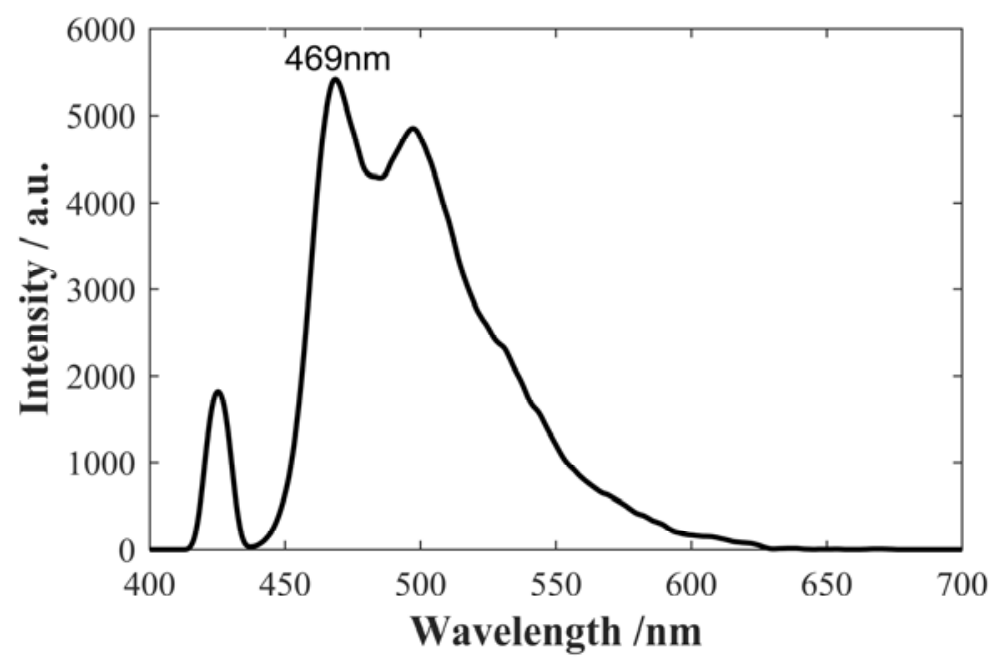

Figure S4 Luminescence spectra and maximum emission wavelength of Ir-1 


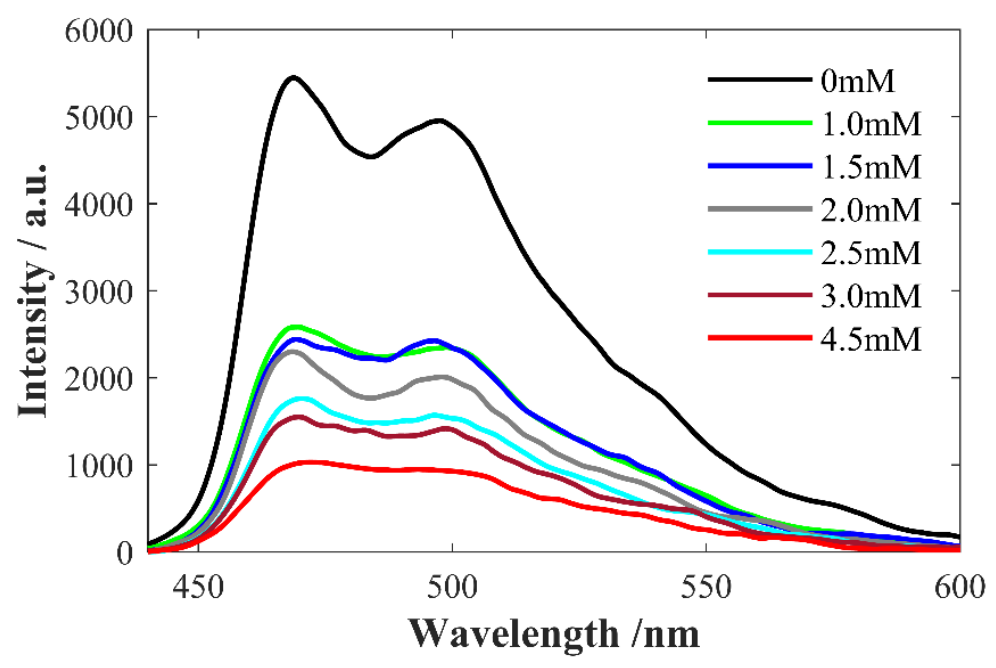

Figure S5 The luminescence spectra of Ir-1 photocatalyst with different concentration of TEA excited at $425 \mathrm{~nm}$.

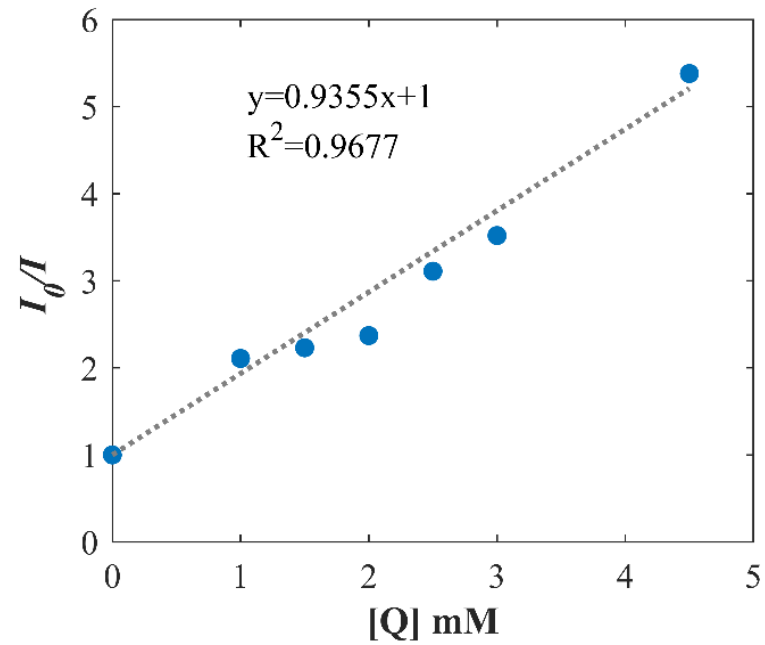

Figure S6 Ir-1 emission quenching by TEA. Linear quenching is observed. 


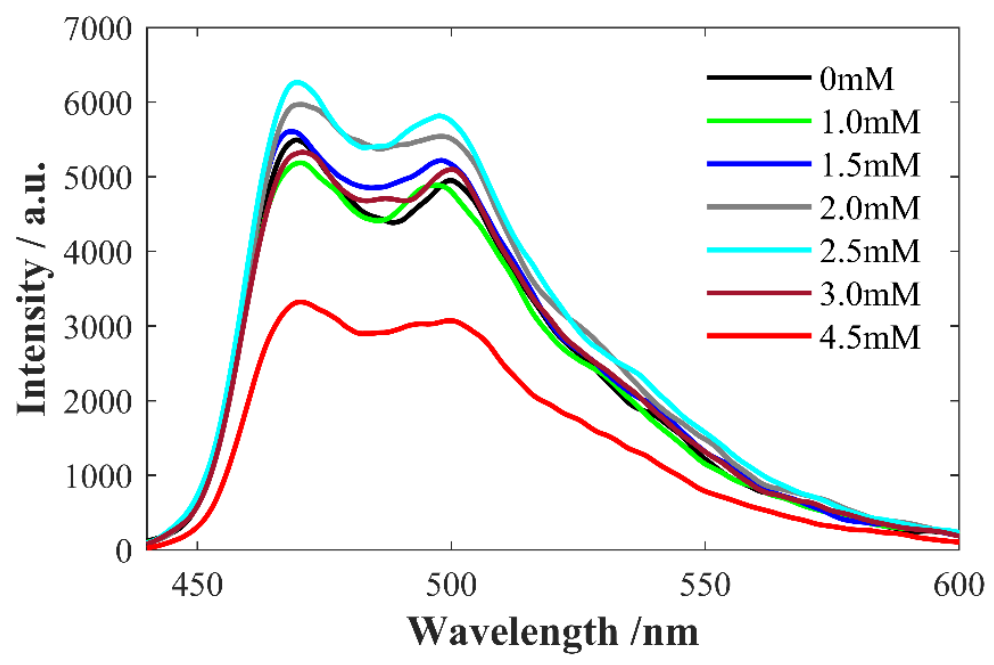

Figure S7 The luminescence spectra of Ir-1 photocatalyst with different concentration of $\mathrm{PPh}_{3}$ excited at $425 \mathrm{~nm}$.

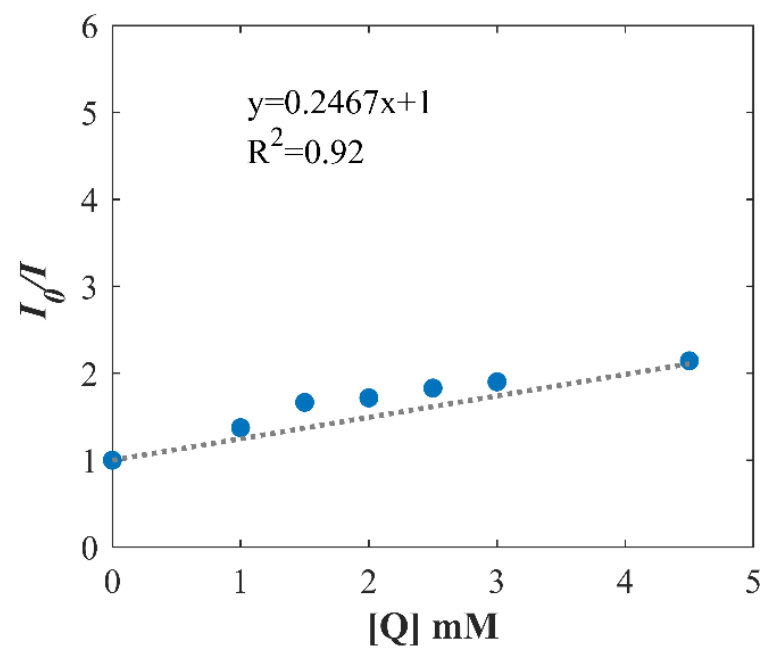

Figure S8 Ir-1 emission quenching by $\mathrm{PPh}_{3}$ Linear quenching is observed. 


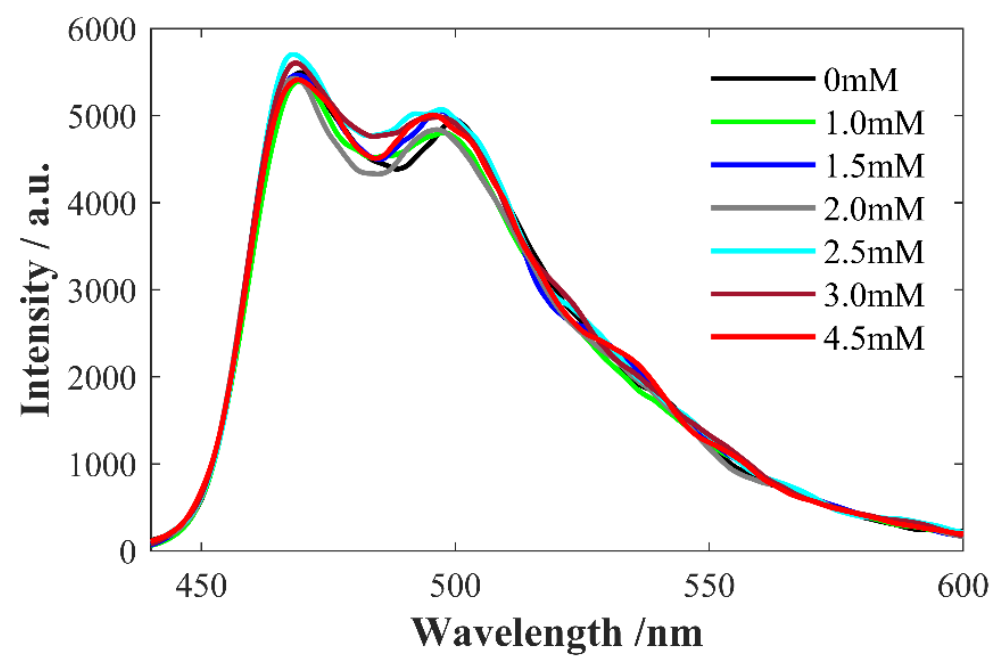

Figure S9 The luminescence spectra of Ir-1 photocatalyst with different concentration of 1 a excited at $425 \mathrm{~nm}$.

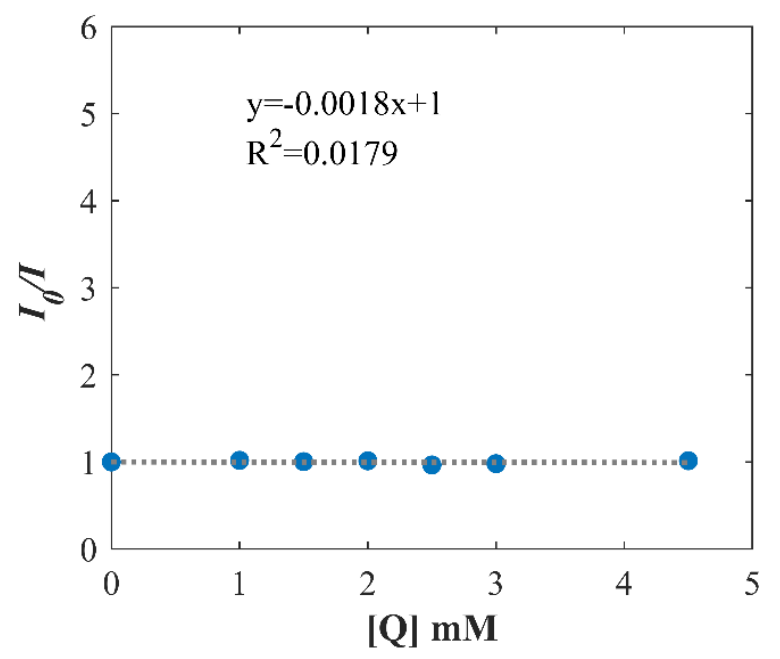

Figure S10 Ir-1 emission quenching by 1 a no quenching effect is observed. 


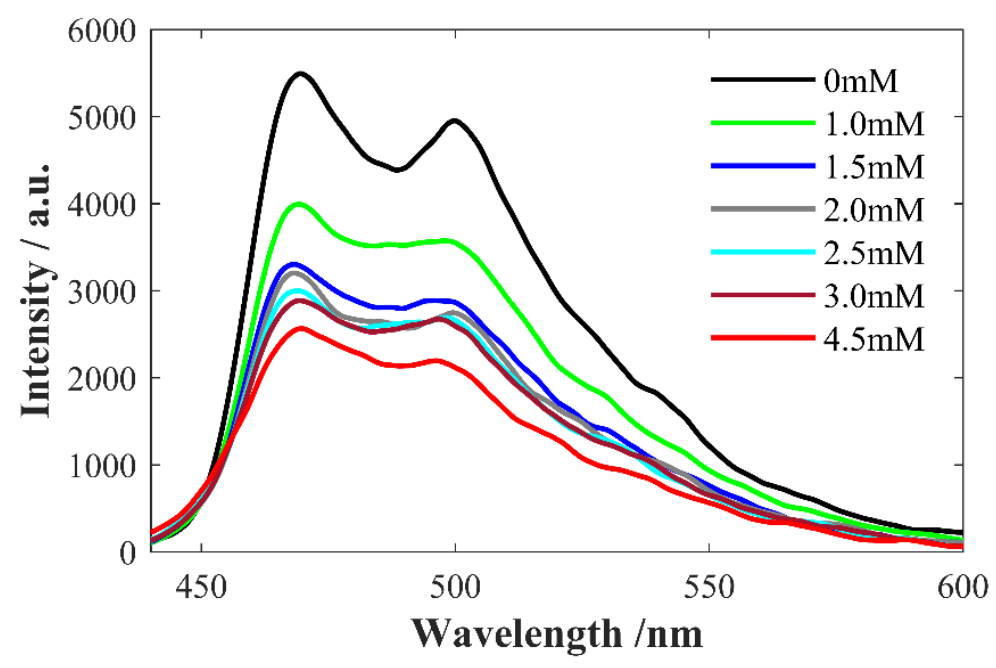

Figure S11 The luminescence spectra of Ir-1 photocatalyst with different concentration of 2 excited at $425 \mathrm{~nm}$.

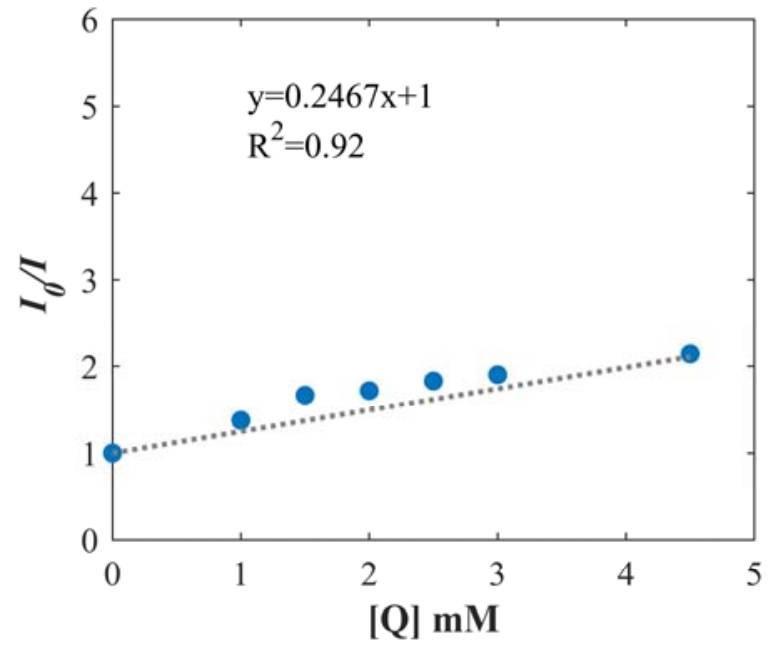

Figure S12 Ir-1 emission quenching by 2 Linear quenching is observed. 
Based on the above experiments and previous studies, we propose a plausible mechanism (Figure S13)

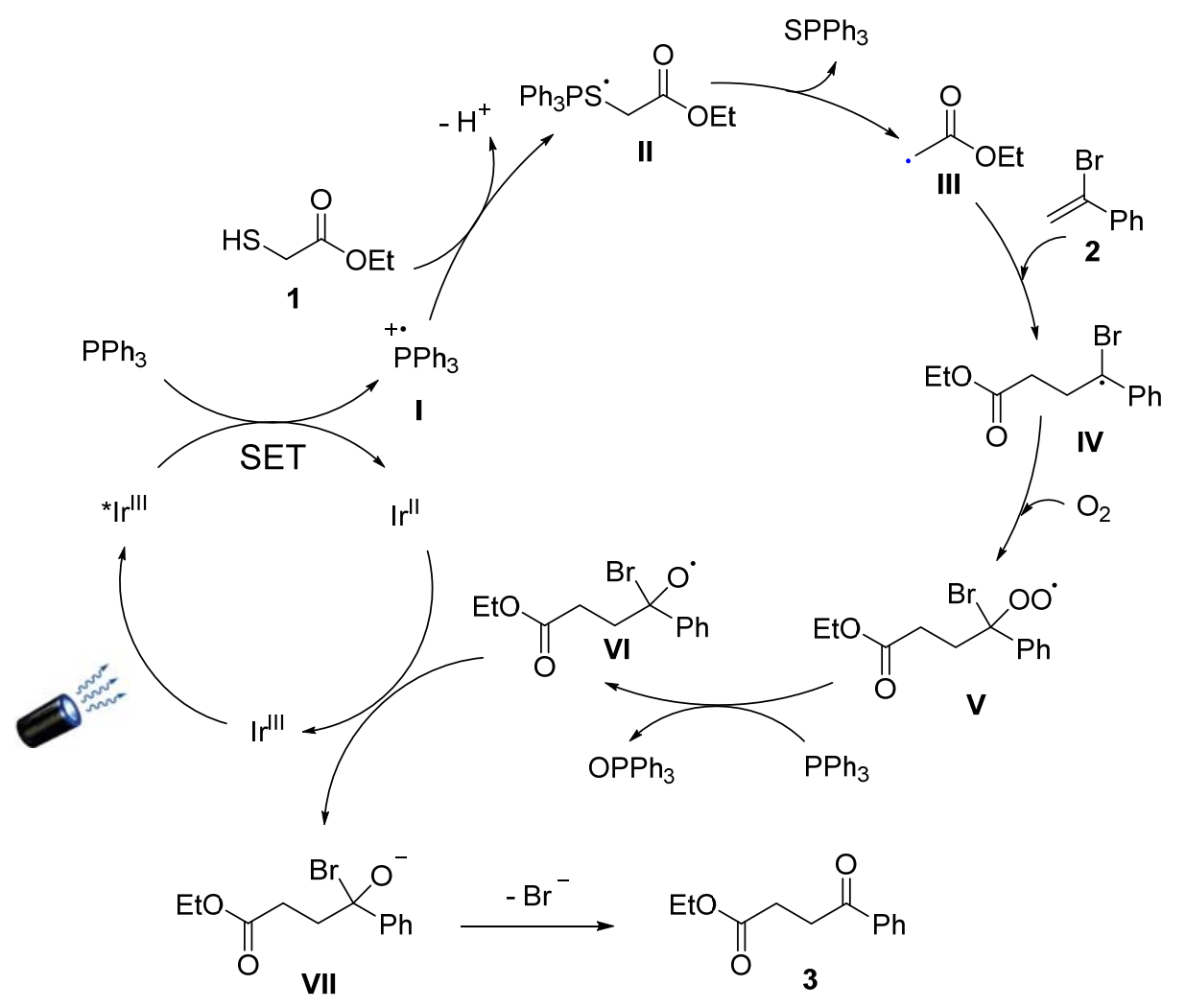

Figure S13 Proposed mechanism 


\title{
3. General Procedures and Experimental Data
}

\section{(a) General procedure A for the synthesis of alkenyl chlorides:}

\author{
$\mathrm{HCl} 1.1$ eq, $\mathrm{RuCl}\left(\mathrm{C}_{5} \mathrm{Me}_{5}\right)$ (cod) $2.5 \mathrm{~mol} \%$ \\ $\mathrm{R}_{\mathrm{R}} \stackrel{\mathrm{PPh}_{3} 0.025 \mathrm{eq}, \mathrm{Et}_{2} \mathrm{O}, \mathrm{N}_{2} \text {, r.t for } 3 \mathrm{~h}}{\longrightarrow} \mathrm{R}_{\mathrm{Cl}}^{\mathrm{R}}$
}

The corresponding alkenyl chlorides were prepared according to literature procedures. ${ }^{5}$

In a Schlenk tube under inert atmosphere, to a solution of 1,2-dichloroethane $(0.5 \mathrm{~mL})$ containing the precatalyst $\mathrm{RuCl}\left(\mathrm{C}_{5} \mathrm{Me}_{5}\right)(\mathrm{cod})(0.025 \mathrm{mmol})$ and $\mathrm{PPh}_{3}(0.025 \mathrm{mmol})$ were added the alkyne $(1.0 \mathrm{mmol}$ ) and $2 \mathrm{~N} \mathrm{HCl}$ (in diethyl ether, 1.1 equiv.). The mixture was stirred at the room temperature for $10 \mathrm{~min}$ to 4 hours. The reaction progress was monitored by TLC analysis. The organic layer was separated, and the aqueous layer was extracted with ether $(4 \times 25 \mathrm{~mL})$. The combined organic layer was washed with $10 \% \mathrm{NaHCO}_{3}$ solution $(2 \times 15 \mathrm{~mL})$, dried over anhydrous $\mathrm{Na}_{2} \mathrm{SO}_{4}$, filtered and concentrated. The residue was used directly for the next step.

(1-chlorovinyl) benzene $[\mathbf{2 a}(\mathbf{X}=\mathbf{C l})]$<smiles>C=C(Cl)c1ccccc1</smiles>

${ }^{1} \mathrm{H}$ NMR $\left(400 \mathrm{MHz}, \mathrm{CDCl}_{3}\right) \delta 7.66(\mathrm{dd}, J=7.6,1.9 \mathrm{~Hz}, 2 \mathrm{H}), 7.45-7.31(\mathrm{~m}, 3 \mathrm{H}), 5.80(\mathrm{~d}, J$ $=1.6 \mathrm{~Hz}, 1 \mathrm{H}), 5.56(\mathrm{~d}, J=1.6 \mathrm{~Hz}, 1 \mathrm{H})$. Analytic data is in accordance with literature data. ${ }^{5}$

2-(1-chlorovinyl) naphthalene $[\mathbf{2} \mathbf{b}(\mathbf{X}=\mathbf{C l})]$<smiles>C=C(Cl)c1ccc2ccccc2c1</smiles>

${ }^{1} \mathrm{H} \mathrm{NMR}\left(400 \mathrm{MHz}, \mathrm{CDCl}_{3}\right) \delta 8.17(\mathrm{~d}, J=1.6 \mathrm{~Hz}, 1 \mathrm{H}), 7.93-7.81(\mathrm{~m}, 3 \mathrm{H}), 7.73(\mathrm{dd}, J=$ 8.7, $1.9 \mathrm{~Hz}, 1 \mathrm{H}), 7.56-7.50(\mathrm{~m}, 2 \mathrm{H}), 5.94(\mathrm{~d}, J=1.9 \mathrm{~Hz}, 1 \mathrm{H}), 5.66(\mathrm{~d}, J=1.9 \mathrm{~Hz}, 1 \mathrm{H})$. Analytic data is in accordance with literature data. ${ }^{6}$ 
1-(1-chlorovinyl)-4-fluorobenzene $[2 \mathbf{c}(\mathbf{X}=\mathbf{C l})]$<smiles>C=C(Cl)c1ccc(F)cc1</smiles>

$\mathrm{H} \mathrm{NMR}\left(400 \mathrm{MHz}, \mathrm{CDCl}_{3}\right) \delta 7.71-7.50(\mathrm{t}, 2 \mathrm{H}), 7.05(\mathrm{t}, J=7.8 \mathrm{~Hz}, 2 \mathrm{H}), 5.70(\mathrm{~d}, 1 \mathrm{H})$, $5.50(\mathrm{~d}, 1 \mathrm{H})$. Analytic data is in accordance with literature data. ${ }^{7}$

1-chloro-4-(1-chlorovinyl) benzene $[\mathbf{2 d}(\mathbf{X}=\mathbf{C l})]$<smiles>C=C(Cl)c1ccc(Cl)cc1</smiles>

${ }^{1} \mathrm{H} \operatorname{NMR}\left(400 \mathrm{MHz}, \mathrm{cdcl}_{3}\right) \delta 7.56(\mathrm{~d}, J=8.5 \mathrm{~Hz}, 2 \mathrm{H}), 7.34(\mathrm{~d}, J=8.5 \mathrm{~Hz}, 2 \mathrm{H}), 5.76(\mathrm{~d}, J=$ $1.5 \mathrm{~Hz}, 1 \mathrm{H}), 5.54(\mathrm{~d}, J=1.5 \mathrm{~Hz}, 1 \mathrm{H})$. Analytic data is in accordance with litera- -ture data. ${ }^{8}$

1-bromo-4-(1-chlorovinyl) benzene $[\mathbf{2 e}(\mathbf{X}=\mathbf{C l})]$<smiles>C=C(Cl)c1ccc(Br)cc1</smiles>

${ }^{1} \mathrm{H}$ NMR $\left(400 \mathrm{MHz}, \mathrm{cdcl}_{3}\right) \delta 7.49(\mathrm{~s}, 4 \mathrm{H}), 5.77(\mathrm{~s}, 1 \mathrm{H}), 5.55(\mathrm{~s}, 1 \mathrm{H})$. Analytic data is in accordance with literature data. ${ }^{5}$

1-(1-chlorovinyl)-4-methyl benzene $[\mathbf{2} \mathbf{f}(\mathbf{X}=\mathbf{C l})]$<smiles>C=C(Cl)c1ccc(C)cc1</smiles>

${ }^{1} \mathrm{H}$ NMR $\left(400 \mathrm{MHz}, \mathrm{CDCl}_{3}\right) \delta 7.52(\mathrm{~d}, J=8.2 \mathrm{~Hz}, 2 \mathrm{H}), 7.17(\mathrm{~d}, J=8.0 \mathrm{~Hz}, 2 \mathrm{H}), 5.72(\mathrm{~d}, J=$ $1.6 \mathrm{~Hz}, 1 \mathrm{H}), 5.47(\mathrm{~d}, J=1.6 \mathrm{~Hz}, 1 \mathrm{H}), 2.37(\mathrm{~s}, 3 \mathrm{H})$. Analytic data is in accord- -ance with literature data. ${ }^{5}$

1-(1-chlorovinyl)-4-ethyl benzene $[\mathbf{2 g}(\mathbf{X}=\mathbf{C l})]$<smiles>C=C(Cl)c1ccc(CC)cc1</smiles>

${ }^{1} \mathrm{H}$ NMR $\left(400 \mathrm{MHz}, \mathrm{CDCl}_{3}\right) \delta 7.58(\mathrm{~d}, J=7.7 \mathrm{~Hz}, 2 \mathrm{H}), 7.22(\mathrm{~d}, J=7.6 \mathrm{~Hz}, 2 \mathrm{H}), 5.75(\mathrm{~s}$, $1 \mathrm{H}), 5.50(\mathrm{~s}, 1 \mathrm{H}), 2.69(\mathrm{dd}, J=14.6,7.2 \mathrm{~Hz}, 2 \mathrm{H}), 1.27(\mathrm{t}, J=7.4 \mathrm{~Hz}, 3 \mathrm{H})$. Analytic data is in accordance with literature data. ${ }^{7}$ 
1-(1-chlorovinyl)-4-propylbenzene $[\mathbf{2 h}(\mathbf{X}=\mathbf{C l})]$<smiles>C=C(Cl)c1ccc(C(C)C)cc1</smiles>

${ }^{1} \mathrm{H} \mathrm{NMR}\left(400 \mathrm{MHz}, \mathrm{CDCl}_{3}\right) \delta 7.58(\mathrm{~d}, J=7.8 \mathrm{~Hz}, 2 \mathrm{H}), 7.20(\mathrm{~d}, J=7.7 \mathrm{~Hz}, 2 \mathrm{H}), 5.76(\mathrm{~s}$, $1 \mathrm{H}), 5.50(\mathrm{~s}, 1 \mathrm{H}), 2.63(\mathrm{t}, J=7.4 \mathrm{~Hz}, 2 \mathrm{H}), 1.67(\mathrm{dt}, J=14.0,7.0 \mathrm{~Hz}, 2 \mathrm{H}), 0.98(\mathrm{t}, J=7.2 \mathrm{~Hz}$, $3 \mathrm{H})$. Analytic data is in accordance with literature data. ${ }^{9}$

1-(1-chlorovinyl)-4-methoxybenzene $[2 \mathbf{i}(\mathbf{X}=\mathbf{C l})]$<smiles>C=C(Cl)c1ccc(OC)cc1</smiles>

${ }^{1} \mathrm{H}$ NMR $\left(400 \mathrm{MHz}, \mathrm{CDCl}_{3}\right) \delta 7.55(\mathrm{~d}, J=9.0 \mathrm{~Hz}, 2 \mathrm{H}), 6.86(\mathrm{~d}, J=8.9 \mathrm{~Hz}, 2 \mathrm{H}), 5.64(\mathrm{~d}, J=$ $1.7 \mathrm{~Hz}, 1 \mathrm{H}), 5.39(\mathrm{~d}, J=1.7 \mathrm{~Hz}, 1 \mathrm{H}), 3.81(\mathrm{~s}, 3 \mathrm{H})$. Analytic data is in accord- -ance with literature data. ${ }^{6}$

4-(1-chlorovinyl)-1,1'-biphenyl $[\mathbf{2 j}(\mathbf{X}=\mathbf{C l})]$<smiles>C=C(Cl)c1ccc(-c2ccccc2)cc1</smiles>

${ }^{1} \mathrm{H}$ NMR $\left(400 \mathrm{MHz}, \mathrm{CDCl}_{3}\right) \delta 7.75-7.70(\mathrm{~m}, 2 \mathrm{H}), 7.64-7.56(\mathrm{~m}, 4 \mathrm{H}), 7.49$ - $7.44(\mathrm{~m}$, $2 \mathrm{H}), 7.41-7.35(\mathrm{~m}, 1 \mathrm{H}), 5.84(\mathrm{~d}, J=1.8 \mathrm{~Hz}, 1 \mathrm{H}), 5.56(\mathrm{~d}, J=1.8 \mathrm{~Hz}, 1 \mathrm{H})$. Analytic data is in accordance with literature data. ${ }^{10}$

1-(1-chlorovinyl)-3-fluorobenzene $[2 \mathbf{k}(\mathbf{X}=\mathbf{C l})]$<smiles>C=C(Cl)c1cccc(F)c1</smiles>

${ }^{1} \mathrm{H}$ NMR $\left(400 \mathrm{MHz}, \mathrm{CDCl}_{3}\right) \delta 7.42(\mathrm{ddd}, J=7.8,1.6,1.1 \mathrm{~Hz}, 1 \mathrm{H}), 7.38-7.30(\mathrm{~m}, 2 \mathrm{H})$, $7.05(\mathrm{tdd}, J=8.3,2.6,0.9 \mathrm{~Hz}, 1 \mathrm{H}), 5.80(\mathrm{~d}, J=1.9 \mathrm{~Hz}, 1 \mathrm{H}), 5.58(\mathrm{~d}, J=1.9 \mathrm{~Hz}, 1 \mathrm{H})$. 
1-chloro-3-(1-chlorovinyl) benzene $[\mathbf{2 1}(\mathbf{X}=\mathbf{C l})]$<smiles>C=C(Cl)c1cccc(Cl)c1</smiles>

${ }^{1} \mathrm{H} \mathrm{NMR}\left(400 \mathrm{MHz}, \mathrm{CDCl}_{3}\right) \delta 7.62(\mathrm{~s}, 1 \mathrm{H}), 7.50(\mathrm{t}, J=8.2 \mathrm{~Hz}, 1 \mathrm{H}), 7.39-7.25(\mathrm{~m}, 2 \mathrm{H})$, $5.79(\mathrm{~s}, 1 \mathrm{H}), 5.57(\mathrm{~s}, 1 \mathrm{H})$. Analytic data is in accordance with literature data. ${ }^{11}$

1-bromo-3-(1-chlorovinyl) benzene $[2 \mathbf{m}(\mathbf{X}=\mathbf{C l})]$<smiles>C=C(Cl)c1cccc(Br)c1</smiles>

${ }^{1} \mathrm{H} \mathrm{NMR}\left(400 \mathrm{MHz}, \mathrm{CDCl}_{3}\right) \delta 7.78(\mathrm{t}, J=1.8 \mathrm{~Hz}, 1 \mathrm{H}), 7.56(\mathrm{ddd}, J=7.9,1.7,1.0 \mathrm{~Hz}, 1 \mathrm{H})$, $7.51-7.45(\mathrm{~m}, 1 \mathrm{H}), 7.23(\mathrm{~d}, J=7.9 \mathrm{~Hz}, 1 \mathrm{H}), 5.78(\mathrm{~d}, J=2.0 \mathrm{~Hz}, 1 \mathrm{H}), 5.57(\mathrm{~d}, J=2.0 \mathrm{~Hz}$, $1 \mathrm{H})$. Analytic data is in accordance with literature data. ${ }^{12}$

1-(1-chlorovinyl)-3-methylbenzene $[\mathbf{2 n}(\mathbf{X}=\mathbf{C l})]$<smiles>C=C(Cl)c1cccc(C)c1</smiles>

${ }^{1} \mathrm{H}$ NMR $\left(400 \mathrm{MHz}, \mathrm{CDCl}_{3}\right) \delta 7.45-7.43(\mathrm{~m}, 2 \mathrm{H}), 7.28-7.24(\mathrm{~m}, 1 \mathrm{H}), 7.18(\mathrm{~d}, J=7.4 \mathrm{~Hz}$, $1 \mathrm{H}), 5.76(\mathrm{~d}, J=1.7 \mathrm{~Hz}, 1 \mathrm{H}), 5.52(\mathrm{~d}, J=1.7 \mathrm{~Hz}, 1 \mathrm{H}), 2.39(\mathrm{~s}, 3 \mathrm{H})$. Analytic data is in accordance with literature data. ${ }^{13}$

1-(1-chlorovinyl)-2-fluorobenzene $[\mathbf{2 o}(\mathbf{X}=\mathbf{C l})]$<smiles>C=C(Cl)c1ccccc1F</smiles>

${ }^{1} \mathrm{H} \mathrm{NMR}\left(400 \mathrm{MHz}, \mathrm{CDCl}_{3}\right) \delta 7.60(\mathrm{dd}, J=8.0,1.2 \mathrm{~Hz}, 1 \mathrm{H}), 7.39(\mathrm{dd}, J=7.7,1.8 \mathrm{~Hz}, 1 \mathrm{H})$, $7.31(\mathrm{td}, J=7.5,1.2 \mathrm{~Hz}, 1 \mathrm{H}), 7.20(\mathrm{td}, J=7.7,1.8 \mathrm{~Hz}, 1 \mathrm{H}), 5.72(\mathrm{~d}, J=1.5 \mathrm{~Hz}, 1 \mathrm{H}), 5.48(\mathrm{~d}$, $J=1.5 \mathrm{~Hz}, 1 \mathrm{H})$. 
1-chloro-2-(1-chlorovinyl)benzene [2p(X=Cl)]<smiles>C=C(Cl)c1ccccc1Cl</smiles>

${ }^{1} \mathrm{H}$ NMR (400 MHz, $\left.\mathrm{CDCl}_{3}\right) \delta 7.43$ - $7.39(\mathrm{~m}, 2 \mathrm{H}), 7.31-7.26(\mathrm{~m}, 2 \mathrm{H}), 5.74(\mathrm{~d}, J=1.5 \mathrm{~Hz}$, $1 \mathrm{H}), 5.52(\mathrm{~d}, J=1.5 \mathrm{~Hz}, 1 \mathrm{H})$. Analytic data is in accordance with literature data. ${ }^{14}$

1-bromo-2-(1-chlorovinyl) benzene $[\mathbf{2 q}(\mathbf{X}=\mathbf{C l})]$<smiles>C=C(Cl)c1ccccc1Br</smiles>

${ }^{1} \mathrm{H} \mathrm{NMR}\left(400 \mathrm{MHz}, \mathrm{CDCl}_{3}\right) \delta 7.44-7.38(\mathrm{~m}, 2 \mathrm{H}), 7.30-7.26(\mathrm{~m}, 2 \mathrm{H}), 5.74(\mathrm{~d}, J=1.5 \mathrm{~Hz}$, $1 \mathrm{H}), 5.52(\mathrm{~d}, J=1.5 \mathrm{~Hz}, 1 \mathrm{H})$. Analytic data is in accordance with literature data. ${ }^{15}$

1-(1-chlorovinyl)-2-methoxybenzene $[\mathbf{2 r}(\mathbf{X}=\mathbf{C l})]$<smiles>C=C(Cl)c1ccccc1OC</smiles>

${ }^{1} \mathrm{H}$ NMR $\left(400 \mathrm{MHz}, \mathrm{cdcl}_{3}\right) \delta 7.48(\mathrm{dd}, J=7.6,1.7 \mathrm{~Hz}, 1 \mathrm{H}), 7.33(\mathrm{ddd}, J=8.3,7.5,1.8 \mathrm{~Hz}$, $1 \mathrm{H}), 7.00-6.91(\mathrm{~m}, 2 \mathrm{H}), 5.73(\mathrm{~d}, J=1.0 \mathrm{~Hz}, 1 \mathrm{H}), 5.71(\mathrm{~d}, J=1.0 \mathrm{~Hz}, 1 \mathrm{H}), 3.89(\mathrm{~s}, 3 \mathrm{H})$. Analytic data is in accordance with literature data. ${ }^{13}$

3-(1-chlorovinyl) thiophene $[\mathbf{2 s}(\mathbf{X}=\mathbf{C l})]$<smiles>C=C(Cl)c1ccsc1</smiles>

${ }^{1} \mathrm{H}$ NMR $\left(400 \mathrm{MHz}, \mathrm{CDCl}_{3}\right) \delta 7.54(\mathrm{dd}, J=2.9,1.1 \mathrm{~Hz}, 1 \mathrm{H}), 7.31(\mathrm{dd}, J=5.1,3.1 \mathrm{~Hz}, 1 \mathrm{H})$, $7.28-7.25(\mathrm{~m}, 1 \mathrm{H}), 5.71(\mathrm{~d}, J=1.7 \mathrm{~Hz}, 1 \mathrm{H}), 5.44(\mathrm{~d}, J=1.7 \mathrm{~Hz}, 1 \mathrm{H})$. Analytic data is in accordance with literature data. ${ }^{5}$ 


\title{
(b) General procedure $B$ for the synthesis of alkenyl bromides:
}

\author{
dibromomethane 5 eq.
}

$\mathrm{N}, \mathrm{N}$-dimethylaniline 3 eq. refluxed at $120^{\circ} \mathrm{C}$

$\mathrm{R} / \mathrm{T}$<smiles>[R]C(=C)Br</smiles>

The corresponding alkenyl bromides were prepared according to literature procedures ${ }^{16}$.

Terminal alkynes $(1.0 \mathrm{mmol})$, dibromomethane $(5.0 \mathrm{mmol})$ and $N, N$-dimethylaniline $(3.0$ mmol) were placed in a Schlenk tube $(10 \mathrm{~mL})$, and the mixture was stirred at $120^{\circ} \mathrm{C}$ for 12 hours with an oil bath. The reaction progress was monitored by TLC analysis. The organic layer was separated, and the aqueous layer was extracted with ether $(4 \times 25 \mathrm{~mL})$. The combined organic layer was washed with $10 \% \mathrm{NaHCO}_{3}$ solution $(2 \times 15 \mathrm{~mL})$, dried over anhydrous $\mathrm{Na}_{2} \mathrm{SO}_{4}$, filtered and concentrated. The residue was used directly for the next step.

2-(1-bromovinyl) naphthalene $[\mathbf{2} \mathbf{b}(\mathbf{X}=\mathbf{B r})]$<smiles>C=C(Br)c1ccc2ccccc2c1</smiles>

${ }^{1} \mathrm{H} \mathrm{NMR}\left(400 \mathrm{MHz}, \mathrm{CDCl}_{3}\right) \delta 8.12(\mathrm{~d}, J=1.4 \mathrm{~Hz}, 1 \mathrm{H}), 7.93-7.78(\mathrm{~m}, 3 \mathrm{H}), 7.72(\mathrm{td}, J=8.8$, $1.9 \mathrm{~Hz}, 1 \mathrm{H}), 7.57-7.50(\mathrm{~m}, 2 \mathrm{H}), 6.29(\mathrm{~d}, J=2.1 \mathrm{~Hz}, 1 \mathrm{H}), 5.91(\mathrm{~d}, J=2.1 \mathrm{~Hz}, 1 \mathrm{H})$. Analytic data is in accordance with literature data. ${ }^{6}$

1-(1-bromovinyl)-4-fluorobenzene [2c $(\mathrm{X}=\mathbf{B r})]$<smiles>C=C(Br)c1ccc(F)cc1</smiles>

${ }^{1} \mathrm{H}$ NMR $\left(400 \mathrm{MHz}, \mathrm{CDCl}_{3}\right) \delta 7.59-7.52(\mathrm{~m}, 2 \mathrm{H}), 7.08-7.02(\mathrm{~m}, 2 \mathrm{H}), 6.04(\mathrm{~d}, J=2.1 \mathrm{~Hz}$, $1 \mathrm{H}), 5.74(\mathrm{~d}, J=2.0 \mathrm{~Hz}, 1 \mathrm{H})$. Analytic data is in accordance with literature data. ${ }^{17}$

1-(1-bromovinyl)-4-chloro benzene [2d(X=Br)]<smiles>C=C(Br)c1ccc(Cl)cc1</smiles>

${ }^{1} \mathrm{H} \mathrm{NMR}\left(400 \mathrm{MHz}, \mathrm{CDCl}_{3}\right) \delta 7.52(\mathrm{~d}, J=8.6 \mathrm{~Hz}, 2 \mathrm{H}), 7.32(\mathrm{~d}, J=8.6 \mathrm{~Hz}, 2 \mathrm{H}), 6.11(\mathrm{~d}, J=$ $2.2 \mathrm{~Hz}, 1 \mathrm{H}), 5.79(\mathrm{~d}, J=2.2 \mathrm{~Hz}, 1 \mathrm{H})$. Analytic data is in accordance with literature data. ${ }^{6}$ 
1-bromo-4-(1-bromovinyl) benzene [2e(X=Br)]<smiles>C=C(Br)c1ccc(Br)cc1</smiles>

${ }^{1} \mathrm{H} \mathrm{NMR}\left(400 \mathrm{MHz}, \mathrm{CDCl}_{3}\right) \delta 7.48(\mathrm{~d}, J=9.3 \mathrm{~Hz}, 4 \mathrm{H}), 6.12(\mathrm{~d}, J=1.5 \mathrm{~Hz}, 1 \mathrm{H}), 5.79(\mathrm{~d}, J=$ $1.6 \mathrm{~Hz}, 1 \mathrm{H})$. Analytic data is in accordance with literature data. ${ }^{6}$

1-(1-bromovinyl)-4-methylbenzene $[\mathbf{2 f}(\mathbf{X}=\mathbf{B r})]$<smiles>C=C(Br)c1ccc(C)cc1</smiles>

${ }^{1} \mathrm{H}$ NMR $\left(400 \mathrm{MHz}, \mathrm{CDCl}_{3}\right) \delta 7.49(\mathrm{~d}, J=8.0 \mathrm{~Hz}, 2 \mathrm{H}), 7.15(\mathrm{~d}, J=7.9 \mathrm{~Hz}, 2 \mathrm{H}), 6.08(\mathrm{~d}, J=$ $1.7 \mathrm{~Hz}, 1 \mathrm{H}), 5.73(\mathrm{~d}, J=1.7 \mathrm{~Hz}, 1 \mathrm{H}), 2.37(\mathrm{~s}, 3 \mathrm{H})$. Analytic data is in accordance with literature data. ${ }^{6}$

1-(1-bromovinyl)-4-ethyl benzene $[\mathbf{2 g}(\mathbf{X}=\mathbf{B r})]$<smiles>C=C(Br)c1ccc(CC)cc1</smiles>

${ }^{1} \mathrm{H}$ NMR $\left(400 \mathrm{MHz}, \mathrm{CDCl}_{3}\right) \delta 7.55(\mathrm{~d}, J=8.2 \mathrm{~Hz}, 2 \mathrm{H}), 7.21(\mathrm{~d}, J=8.1 \mathrm{~Hz}, 2 \mathrm{H}), 6.11(\mathrm{~d}, J=$ $1.9 \mathrm{~Hz}, 1 \mathrm{H}), 5.76(\mathrm{~d}, J=1.9 \mathrm{~Hz}, 1 \mathrm{H}), 2.70(\mathrm{q}, J=7.6 \mathrm{~Hz}, 2 \mathrm{H}), 1.28(\mathrm{t}, J=7.6 \mathrm{~Hz}, 3 \mathrm{H})$.

Analytic data is in accordance with literature data. ${ }^{18}$

1-(1-bromovinyl)-4-propylbenzene $[\mathbf{2 h}(\mathbf{X}=\mathbf{B r})]$<smiles>C=C(Br)c1ccc(C(C)C)cc1</smiles>

${ }^{1} \mathrm{H} \mathrm{NMR}\left(400 \mathrm{MHz}, \mathrm{CDCl}_{3}\right) \delta 7.51(\mathrm{~d}, J=7.8 \mathrm{~Hz}, 2 \mathrm{H}), 7.16(\mathrm{~d}, J=7.9 \mathrm{~Hz}, 2 \mathrm{H}), 6.09(\mathrm{~s}$, $1 \mathrm{H}), 5.73(\mathrm{~s}, 1 \mathrm{H}), 2.60(\mathrm{t}, J=7.4 \mathrm{~Hz}, 2 \mathrm{H}), 1.64(\mathrm{dq}, J=14.5,7.2 \mathrm{~Hz}, 2 \mathrm{H}), 0.95(\mathrm{t}, J=7.2 \mathrm{~Hz}$, $3 \mathrm{H})$. Analytic data is in accordance with literature data. ${ }^{10}$ 
1-(1-bromovinyl)-4-methoxybenzene [ $\mathbf{2 i}(\mathbf{X}=\mathbf{B r})]$<smiles>C=C(Br)c1ccc(OC)cc1</smiles>

${ }^{1} \mathrm{H} \mathrm{NMR}\left(400 \mathrm{MHz}, \mathrm{CDCl}_{3}\right) \delta 7.54(\mathrm{~d}, J=8.9 \mathrm{~Hz}, 2 \mathrm{H}), 6.86(\mathrm{~d}, J=8.9 \mathrm{~Hz}, 2 \mathrm{H}), 6.01(\mathrm{~d}, J=$ $2.0 \mathrm{~Hz}, 1 \mathrm{H}), 5.67(\mathrm{~d}, J=2.0 \mathrm{~Hz}, 1 \mathrm{H}), 3.83(\mathrm{~s}, 3 \mathrm{H})$. Analytic data is in accordance with literature data. ${ }^{6}$

4-(1-bromovinyl)-1,1'-biphenyl [2j(X=Br)]<smiles>C=C(Br)c1ccc(-c2ccccc2)cc1</smiles>

${ }^{1} \mathrm{H}$ NMR $\left(400 \mathrm{MHz}, \mathrm{CDCl}_{3}\right) \delta 7.72-7.66(\mathrm{~m}, 2 \mathrm{H}), 7.64-7.57(\mathrm{~m}, 4 \mathrm{H}), 7.47$ (dd, J = 10.2, $4.8 \mathrm{~Hz}, 2 \mathrm{H}), 7.41-7.36(\mathrm{~m}, 1 \mathrm{H}), 6.19(\mathrm{~d}, J=2.0 \mathrm{~Hz}, 1 \mathrm{H}), 5.82(\mathrm{~d}, J=2.0 \mathrm{~Hz}, 1 \mathrm{H})$. Analytic data is in accordance with literature data. ${ }^{19}$

1-(1-bromovinyl)-3-fluorobenzene [ $2 \mathbf{k}(\mathrm{X}=\mathbf{B r})]$<smiles>C=C(Br)c1cccc(F)c1</smiles>

${ }^{1} \mathrm{H}$ NMR $\left(400 \mathrm{MHz}, \mathrm{CDCl}_{3}\right) \delta 7.37(\mathrm{~d}, J=7.9 \mathrm{~Hz}, 1 \mathrm{H}), 7.34-7.28(\mathrm{~m}, 2 \mathrm{H}), 7.03(\mathrm{t}, J=8.1$ $\mathrm{Hz}, 1 \mathrm{H}), 6.15(\mathrm{~d}, J=2.1 \mathrm{~Hz}, 1 \mathrm{H}), 5.82(\mathrm{~d}, J=2.1 \mathrm{~Hz}, 1 \mathrm{H})$. Analytic data is in accordance with literature data. ${ }^{20}$

1-chloro-3- (1-bromovinyl) benzene [21(X=Br)]<smiles>C=C(Br)c1cccc(Cl)c1</smiles>

${ }^{1} \mathrm{H}$ NMR $\left(400 \mathrm{MHz}, \mathrm{CDCl}_{3}\right) \delta 7.57(\mathrm{~d}, J=1.8 \mathrm{~Hz}, 1 \mathrm{H}), 7.45(\mathrm{dd}, J=7.2,1.7 \mathrm{~Hz}, 1 \mathrm{H}), 7.32$ $-7.22(\mathrm{~m}, 3 \mathrm{H}), 6.12(\mathrm{~d}, J=2.1 \mathrm{~Hz}, 1 \mathrm{H}), 5.81(\mathrm{~d}, J=2.1 \mathrm{~Hz}, 1 \mathrm{H})$. Analytic data is in accordance with literature data. ${ }^{21}$ 
1-bromo-3-(1-bromovinyl)benzene [ $2 \mathbf{m}(\mathrm{X}=\mathbf{B r})]$<smiles>C=C(Br)c1cccc(Br)c1</smiles>

${ }^{1} \mathrm{H} \mathrm{NMR}\left(400 \mathrm{MHz}, \mathrm{CDCl}_{3}\right) \delta 7.73(\mathrm{t}, 1 \mathrm{H}), 7.51(\mathrm{t}, J=7.4 \mathrm{~Hz}, 1 \mathrm{H}), 7.45(\mathrm{~s}, 1 \mathrm{H}), 7.21(\mathrm{dd}$, $J=7.6,4.9 \mathrm{~Hz}, 1 \mathrm{H}), 6.13(\mathrm{~d}, J=2.1 \mathrm{~Hz}, 1 \mathrm{H}), 5.82(\mathrm{~d}, J=2.1 \mathrm{~Hz}, 1 \mathrm{H})$. Analytic data is in accordance with literature data. ${ }^{12}$

1-(1-bromovinyl)-3-methylbenzene $[\mathbf{2 n}(\mathbf{X}=\mathbf{B r})]$<smiles>C=C(Br)c1cccc(C)c1</smiles>

${ }^{1} \mathrm{H}$ NMR $\left(400 \mathrm{MHz}, \mathrm{CDCl}_{3}\right) \delta 7.45-7.38(\mathrm{~m}, 2 \mathrm{H}), 7.25(\mathrm{dd}, J=8.8,6.4 \mathrm{~Hz}, 1 \mathrm{H}), 7.16(\mathrm{~d}, J$ $=7.7 \mathrm{~Hz}, 1 \mathrm{H}), 6.11(\mathrm{~d}, J=1.9 \mathrm{~Hz}, 1 \mathrm{H}), 5.78(\mathrm{~d}, J=1.9 \mathrm{~Hz}, 1 \mathrm{H}), 2.39(\mathrm{~s}, 3 \mathrm{H})$. Analytic data is in accordance with literature data. ${ }^{22}$

1-(1-bromovinyl)-2-fluorobenzene [2o(X=Br)]<smiles>C=C(Br)c1ccccc1F</smiles>

${ }^{1} \mathrm{H}$ NMR $\left(400 \mathrm{MHz}, \mathrm{CDCl}_{3}\right) \delta 7.53(\mathrm{td}, J=7.7,1.8 \mathrm{~Hz}, 1 \mathrm{H}), 7.32-7.29(\mathrm{~m}, 1 \mathrm{H}), 7.15(\mathrm{dd}$, $J=7.7,6.6 \mathrm{~Hz}, 1 \mathrm{H}), 7.06(\mathrm{ddd}, J=5.7,3.9,1.1 \mathrm{~Hz}, 1 \mathrm{H}), 6.16-6.11(\mathrm{~m}, 1 \mathrm{H}), 6.04-6.00(\mathrm{~m}$, $1 \mathrm{H})$. Analytic data is in accordance with literature data. ${ }^{20}$

1-(1-bromovinyl)-2-chlorobenzene $[\mathbf{2} \mathbf{p}(\mathrm{X}=\mathbf{B r})]$<smiles>C=C(Br)c1ccccc1Cl</smiles>

${ }^{1} \mathrm{H}$ NMR (400 MHz, CDCl $) \delta 7.39(\mathrm{dt}, J=9.0,5.2 \mathrm{~Hz}, 2 \mathrm{H}), 7.30-7.20(\mathrm{~m}, 2 \mathrm{H}), 5.96(\mathrm{~d}, J$ $=1.5 \mathrm{~Hz}, 1 \mathrm{H}), 5.88(\mathrm{~d}, J=1.5 \mathrm{~Hz}, 1 \mathrm{H})$. Analytic data is in accordance with literature data. ${ }^{15}$ 
1-bromo-2-(1-bromovinyl) benzene $[\mathbf{2 q}(\mathbf{X}=\mathbf{B r})]$

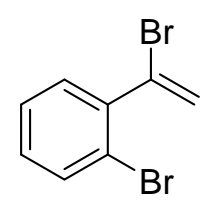

${ }^{1} \mathrm{H}$ NMR $\left(400 \mathrm{MHz}, \mathrm{CDCl}_{3}\right) \delta 7.60-7.57(\mathrm{~m}, 1 \mathrm{H}), 7.37(\mathrm{dd}, J=7.7,1.6 \mathrm{~Hz}, 1 \mathrm{H}), 7.30(\mathrm{td}$, $J=7.5,1.0 \mathrm{~Hz}, 1 \mathrm{H}), 7.21-7.16(\mathrm{~m}, 1 \mathrm{H}), 5.94(\mathrm{~d}, J=1.7 \mathrm{~Hz}, 1 \mathrm{H}), 5.85(\mathrm{~d}, J=1.7 \mathrm{~Hz}, 1 \mathrm{H})$.

1-(1-bromovinyl)-2-methoxybenzene [2r(X=Br)]<smiles>C=C(Br)c1ccccc1OC</smiles>

${ }^{1} \mathrm{H}$ NMR $\left(400 \mathrm{MHz}, \mathrm{CDCl}_{3}\right) \delta 7.38(\mathrm{~d}, J=7.5 \mathrm{~Hz}, 1 \mathrm{H}), 7.32(\mathrm{t}, J=7.8 \mathrm{~Hz}, 1 \mathrm{H}), 6.98-6.88$ $(\mathrm{m}, 2 \mathrm{H}), 5.99(\mathrm{~s}, 1 \mathrm{H}), 5.92(\mathrm{~s}, 1 \mathrm{H}), 3.89(\mathrm{~s}, 3 \mathrm{H})$. Analytic data is in accordance with literature data. ${ }^{23}$

3-(1-bromovinyl) thiophene $[\mathbf{2 s}(\mathbf{X}=\mathbf{B r})]$<smiles>C=C(Br)c1ccsc1</smiles>

${ }^{1} \mathrm{H} \mathrm{NMR}\left(400 \mathrm{MHz}, \mathrm{CDCl}_{3}\right) \delta 7.51(\mathrm{dd}, J=3.0,1.3 \mathrm{~Hz}, 1 \mathrm{H}), 7.32(\mathrm{dd}, J=5.1,3.1 \mathrm{~Hz}, 1 \mathrm{H})$, $7.28-7.24(\mathrm{~m}, 1 \mathrm{H}), 6.11(\mathrm{~d}, J=2.1 \mathrm{~Hz}, 1 \mathrm{H}), 5.69(\mathrm{~d}, J=2.1 \mathrm{~Hz}, 1 \mathrm{H})$. Analytic data is in accordance with literature data. ${ }^{24}$ 


\section{(c) General procedure $\mathrm{C}$ for alkenyl iodides.}

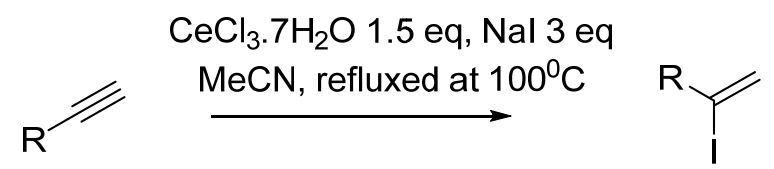

The corresponding alkenyl iodides were prepared according to literature procedures. ${ }^{25}$

To a stirred suspension of alkyne $(1.0 \mathrm{mmol})$ and sodium iodide $(0.45 \mathrm{~g}, 3.0 \mathrm{mmol})$ in 15 $\mathrm{mL} \mathrm{MeCN}$ was added cerium (III) chloride heptahydrate $(0.56 \mathrm{~g}, 1.5 \mathrm{mmol})$, and the resulting mixture was stirred at reflux with an oil bath until the disappearance of the starting alkyne. The reaction progress was monitored by TLC analysis. The reaction mixture was diluted with ether and treated with $0.5 \mathrm{~N} \mathrm{HCl}(15 \mathrm{~mL})$. The organic layer was separated, and the aqueous layer was extracted with ether $(4 \times 25 \mathrm{~mL})$. The combined organic layer was washed with $10 \% \mathrm{NaHCO}_{3}$ solution $(2 \times 15 \mathrm{~mL})$, dried over anhydrous $\mathrm{Na}_{2} \mathrm{SO}_{4}$, filtered and concentrated. The residue was used directly for the next step.

(1-iodovinyl) benzene $[\mathbf{2} \mathbf{a}(\mathbf{X}=\mathbf{I})]$<smiles>C=C(I)c1ccccc1</smiles>

$1 \mathrm{H} \mathrm{NMR}\left(400 \mathrm{MHz}, \mathrm{CDCl}_{3}\right) \delta 7.55-7.46(\mathrm{~m}, 2 \mathrm{H}), 7.40-7.28(\mathrm{~m}, 3 \mathrm{H}), 6.47(\mathrm{~d}, J=1.7$ $\mathrm{Hz}, 1 \mathrm{H}), 6.09(\mathrm{~d}, J=1.7 \mathrm{~Hz}, 1 \mathrm{H})$. Analytic data is in accordance with literature data. ${ }^{5}$

2-(1-iodovinyl) naphthalene $[\mathbf{2} \mathbf{b}(\mathbf{X}=\mathbf{I})]$<smiles>C=C(I)c1ccc2ccccc2c1</smiles>

${ }^{1} \mathrm{H}$ NMR $\left(400 \mathrm{MHz}, \mathrm{CDCl}_{3}\right) \delta 7.98(\mathrm{~s}, 1 \mathrm{H}), 7.85(\mathrm{dd}, J=11.5,5.2 \mathrm{~Hz}, 2 \mathrm{H}), 7.71(\mathrm{dd}, J=$ $49.9,8.4 \mathrm{~Hz}, 2 \mathrm{H}), 7.51(\mathrm{~d}, J=3.5 \mathrm{~Hz}, 2 \mathrm{H}), 6.61(\mathrm{~d}, 1 \mathrm{H}), 6.19(\mathrm{~d}, 1 \mathrm{H})$. Analytic data is in accordance with literature data. ${ }^{26}$

1-fluoro-4-(1-iodovinyl) benzene [ $2 \mathbf{c}(\mathbf{X}=\mathbf{I})]$<smiles>C=C(I)c1ccc(F)cc1</smiles>

${ }^{1} \mathrm{H} \mathrm{NMR}\left(400 \mathrm{MHz}, \mathrm{CDCl}_{3}\right) \delta 7.48(\mathrm{t}, 2 \mathrm{H}), 7.00(\mathrm{t}, J=7.8 \mathrm{~Hz}, 2 \mathrm{H}), 6.40(\mathrm{~d}, 1 \mathrm{H}), 6.06(\mathrm{~d}$, $1 \mathrm{H})$. Analytic data is in accordance with literature data. ${ }^{17}$ 
1-chloro-4-(1-iodovinyl) benzene $[\mathbf{2 d}(\mathbf{X}=\mathbf{I})]$<smiles>C=C(I)c1ccc(Cl)cc1</smiles>

${ }^{1} \mathrm{H} \mathrm{NMR}\left(400 \mathrm{MHz}, \mathrm{CDCl}_{3}\right) \delta 7.45(\mathrm{~d}, J=7.9 \mathrm{~Hz}, 2 \mathrm{H}), 7.29(\mathrm{~s}, 2 \mathrm{H}), 6.45(\mathrm{~s}, 1 \mathrm{H}), 6.09$ (s, $1 \mathrm{H})$. Analytic data is in accordance with literature data. ${ }^{27}$

1-bromo-4-(1-iodovinyl) benzene $[\mathbf{2 e}(\mathbf{X}=\mathbf{I})]$<smiles>C=C(I)c1ccc(Br)cc1</smiles>

${ }^{1} \mathrm{H} \mathrm{NMR}\left(400 \mathrm{MHz}, \mathrm{CDCl}_{3}\right) \delta 7.44(\mathrm{~d}, J=8.7 \mathrm{~Hz}, 2 \mathrm{H}), 7.38(\mathrm{~d}, J=8.7 \mathrm{~Hz}, 2 \mathrm{H}), 6.46(\mathrm{~d}, J=$ $1.8 \mathrm{~Hz}, 1 \mathrm{H}), 6.09(\mathrm{~d}, J=1.8 \mathrm{~Hz}, 1 \mathrm{H})$. Analytic data is in accordance with literature data. ${ }^{28}$

1-(1-iodovinyl)-4-methylbenzene $[\mathbf{2} \mathbf{f}(\mathbf{X}=\mathbf{I})]$<smiles>C=C(I)c1ccc(C)cc1</smiles>

${ }^{1} \mathrm{H} \mathrm{NMR}\left(400 \mathrm{MHz}, \mathrm{CDCl}_{3}\right) \delta 7.41(\mathrm{~d}, J=8.2 \mathrm{~Hz}, 2 \mathrm{H}), 7.11(\mathrm{~d}, J=8.0 \mathrm{~Hz}, 2 \mathrm{H}), 6.43(\mathrm{~d}, J=$ $1.6 \mathrm{~Hz}, 1 \mathrm{H}), 6.03(\mathrm{~d}, J=1.6 \mathrm{~Hz}, 1 \mathrm{H}), 2.35(\mathrm{~s}, 3 \mathrm{H})$. Analytic data is in accord- -ance with literature data. ${ }^{27}$

1-ethyl-4-(1-iodovinyl) benzene $[\mathbf{2 g}(\mathbf{X}=\mathbf{I})]$<smiles>C=C(I)c1ccc(CC)cc1</smiles>

${ }^{1} \mathrm{H} \mathrm{NMR}\left(400 \mathrm{MHz}, \mathrm{CDCl}_{3}\right) \delta 7.45(\mathrm{~d}, J=8.3 \mathrm{~Hz}, 2 \mathrm{H}), 7.15(\mathrm{~d}, J=8.3 \mathrm{~Hz}, 2 \mathrm{H}), 6.45(\mathrm{~d}, J=$ $1.6 \mathrm{~Hz}, 1 \mathrm{H}), 6.05(\mathrm{~d}, J=1.7 \mathrm{~Hz}, 1 \mathrm{H}), 2.71-2.63(\mathrm{~m}, 2 \mathrm{H}), 1.25(\mathrm{t}, J=7.6 \mathrm{~Hz}, 3 \mathrm{H})$.

1-(1-iodovinyl)-4-propylbenzene $[\mathbf{2 h}(\mathbf{X}=\mathbf{I})]$<smiles>C=C(I)c1ccc(C(C)C)cc1</smiles>

${ }^{1} \mathrm{H}$ NMR $\left(400 \mathrm{MHz}, \mathrm{CDCl}_{3}\right) \delta 7.43(\mathrm{~d}, J=8.3 \mathrm{~Hz}, 2 \mathrm{H}), 7.12(\mathrm{~d}, J=8.4 \mathrm{~Hz}, 2 \mathrm{H}), 6.44(\mathrm{~d}, J=$ $1.7 \mathrm{~Hz}, 1 \mathrm{H}), 6.04(\mathrm{~d}, J=1.7 \mathrm{~Hz}, 1 \mathrm{H}), 2.64-2.52(\mathrm{~m}, 2 \mathrm{H}), 1.65(\mathrm{dt}, J=15.0,7.4 \mathrm{~Hz}, 2 \mathrm{H})$, $0.95(\mathrm{t}, J=7.3 \mathrm{~Hz}, 3 \mathrm{H})$. Analytic data is in accordance with literature data. ${ }^{10}$ 
1-(1-iodovinyl)-4-methoxybenzene $[2 \mathbf{i}(\mathbf{X}=\mathbf{I})]$<smiles>C=C(I)c1ccc(OC)cc1</smiles>

${ }^{1} \mathrm{H} \mathrm{NMR}\left(400 \mathrm{MHz}, \mathrm{CDCl}_{3}\right) \delta 7.35(\mathrm{~d}, J=4.7 \mathrm{~Hz}, 2 \mathrm{H}), 6.72(\mathrm{~d}, J=4.7 \mathrm{~Hz}, 2 \mathrm{H}), 6.28(\mathrm{~d}, J=$ $1.8 \mathrm{~Hz}, 1 \mathrm{H}), 5.87(\mathrm{~d}, J=1.8 \mathrm{~Hz}, 1 \mathrm{H}), 3.71(\mathrm{~s}, 3 \mathrm{H})$. Analytic data is in accordance with literature data. ${ }^{28}$

4-(1-iodovinyl)-1,1'-biphenyl [2j(X=I)]<smiles>C=C(I)c1ccc(-c2ccccc2)cc1</smiles>

${ }^{1} \mathrm{H} \mathrm{NMR}\left(400 \mathrm{MHz}, \mathrm{CDCl}_{3}\right) \delta 7.56(\mathrm{dt}, J=24.9,10.1 \mathrm{~Hz}, 6 \mathrm{H}), 7.46(\mathrm{t}, J=7.7 \mathrm{~Hz}, 2 \mathrm{H}), 7.38$ $(\mathrm{dd}, J=11.2,4.2 \mathrm{~Hz}, 1 \mathrm{H}), 6.53(\mathrm{~d}, J=1.1 \mathrm{~Hz}, 1 \mathrm{H}), 6.12(\mathrm{~d}, J=1.1 \mathrm{~Hz}, 1 \mathrm{H})$. Analytic data is in accordance with literature data. ${ }^{29}$

1-fluoro-3-(1-iodovinyl) benzene $[2 \mathbf{k}(\mathbf{X}=\mathbf{I})]$<smiles>C=C(I)c1cccc(F)c1</smiles>

${ }^{1} \mathrm{H} \mathrm{NMR}\left(400 \mathrm{MHz}, \mathrm{CDCl}_{3}\right) \delta 7.29(\mathrm{dd}, J=6.4,4.6 \mathrm{~Hz}, 2 \mathrm{H}), 7.22(\mathrm{dd}, J=11.7,1.6 \mathrm{~Hz}, 1 \mathrm{H})$, $7.02-6.96(\mathrm{~m}, 1 \mathrm{H}), 6.50(\mathrm{~d}, J=1.8 \mathrm{~Hz}, 1 \mathrm{H}), 6.12(\mathrm{~d}, J=1.8 \mathrm{~Hz}, 1 \mathrm{H})$. Analytic data is in accordance with literature data. ${ }^{29}$

1-chloro-3- (1-iodovinyl) benzene $[\mathbf{2 l}(\mathbf{X}=\mathbf{I})]$<smiles>C=C(I)c1cccc(Cl)c1</smiles>

${ }^{1} \mathrm{H} \mathrm{NMR}\left(400 \mathrm{MHz} \mathrm{cdcl}_{3}\right) \delta 7.48(\mathrm{~d}, J=7.2 \mathrm{~Hz}, 1 \mathrm{H}), 7.40-7.32(\mathrm{~m}, 2 \mathrm{H}), 7.25-7.22(\mathrm{~m}$, $1 \mathrm{H}), 6.48(\mathrm{~d}, J=1.3 \mathrm{~Hz}, 1 \mathrm{H}), 6.11(\mathrm{~d}, J=1.3 \mathrm{~Hz}, 1 \mathrm{H})$. Analytic data is in accordance with literature data. $[\mathbf{2 m}(\mathbf{X}=\mathbf{I})]$ 
1-bromo-3-(1-iodovinyl)benzene $[\mathbf{2 m}(\mathbf{X}=\mathbf{I})]$<smiles>C=C(I)c1cccc(Br)c1</smiles>

${ }^{1} \mathrm{H} \mathrm{NMR}\left(400 \mathrm{MHz}, \mathrm{CDCl}_{3}\right) \delta 7.65(\mathrm{t}, J=1.9 \mathrm{~Hz}, 1 \mathrm{H}), 7.45-7.40(\mathrm{~m}, 2 \mathrm{H}), 7.19(\mathrm{t}, J=7.9$ $\mathrm{Hz}, 1 \mathrm{H}), 6.48(\mathrm{~d}, J=1.9 \mathrm{~Hz}, 1 \mathrm{H}), 6.11(\mathrm{~d}, J=1.9 \mathrm{~Hz}, 1 \mathrm{H})$.

1-(1-iodovinyl)-3-methylbenzene $[\mathbf{2 n}(\mathbf{X}=\mathbf{I})]$<smiles>C=C(I)c1cccc(C)c1</smiles>

${ }^{1} \mathrm{H}$ NMR $\left(400 \mathrm{MHz}, \mathrm{CDCl}_{3}\right) \delta$ 7.33-7.30 (m, 2H), 7.24-7.18 (m, $\left.1 \mathrm{H}\right), 7.11(\mathrm{~d}, J=7.6 \mathrm{~Hz}$, $1 \mathrm{H}), 6.45(\mathrm{~d}, J=1.6 \mathrm{~Hz}, 1 \mathrm{H}), 6.07(\mathrm{~d}, J=1.6 \mathrm{~Hz}, 1 \mathrm{H}), 2.37(\mathrm{~s}, 3 \mathrm{H})$. Analytic data is in accordance with literature data. ${ }^{27}$

1-fluoro-2-(1-iodovinyl)benzene $[\mathbf{2 o}(\mathbf{X}=\mathbf{I})]$<smiles>C=C(I)c1ccccc1F</smiles>

${ }^{1} \mathrm{H}$ NMR $\left(400 \mathrm{MHz}, \mathrm{CDCl}_{3}\right) \delta 7.38(\mathrm{td}, J=7.7,1.8 \mathrm{~Hz}, 1 \mathrm{H}), 7.31-7.24(\mathrm{~m}, 1 \mathrm{H}), 7.14-$ $7.07(\mathrm{~m}, 1 \mathrm{H}), 7.07-6.97(\mathrm{~m}, 1 \mathrm{H}), 6.48-6.40(\mathrm{~m}, 1 \mathrm{H}), 6.29(\mathrm{~d}, J=1.5 \mathrm{~Hz}, 1 \mathrm{H})$. Analytic data is in accordance with literature data. ${ }^{29}$

1-chloro-2-(1-iodovinyl) benzene $[\mathbf{2} \mathbf{p}(\mathbf{X}=\mathbf{I})]$<smiles>C=C(I)c1ccccc1Cl</smiles>

${ }^{1} \mathrm{H} \mathrm{NMR}\left(400 \mathrm{MHz}, \mathrm{CDCl}_{3}\right) \delta 7.35$ (dd, $\left.J=5.7,3.6 \mathrm{~Hz}, 1 \mathrm{H}\right), 7.30(\mathrm{dd}, J=5.7,3.7 \mathrm{~Hz}, 1 \mathrm{H})$, $7.23(\mathrm{dd}, J=5.9,3.5 \mathrm{~Hz}, 2 \mathrm{H}), 6.24(\mathrm{~d}, J=1.4 \mathrm{~Hz}, 1 \mathrm{H}), 6.22(\mathrm{~d}, J=1.4 \mathrm{~Hz}, 1 \mathrm{H})$. Analytic data is in accordance with literature data. ${ }^{31}$ 
1-bromo-2-(1-iodovinyl) benzene $[\mathbf{2 q}(\mathbf{X}=\mathbf{I})]$

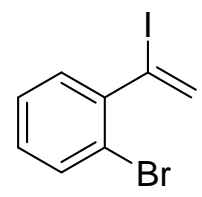

${ }^{1} \mathrm{H} \mathrm{NMR}\left(400 \mathrm{MHz}, \mathrm{CDCl}_{3}\right) \delta 7.55(\mathrm{~d}, J=8.5 \mathrm{~Hz}, 1 \mathrm{H}), 7.33-7.27(\mathrm{~m}, 2 \mathrm{H}), 7.14$ (ddd $J=$ 8.1, 6.8, $2.4 \mathrm{~Hz}, 1 \mathrm{H}), 6.21(\mathrm{~d}, J=1.5 \mathrm{~Hz}, 1 \mathrm{H}), 6.19(\mathrm{~d}, J=1.5 \mathrm{~Hz}, 1 \mathrm{H})$. Analytic data is in accordance with literature data. ${ }^{32}$

1-(1-iodovinyl)-2-methoxybenzene [2r(X=I)]

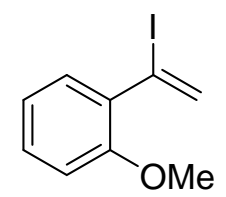

${ }^{1} \mathrm{H}$ NMR (400 MHz, $\left.\mathrm{CDCl}_{3}\right) \delta 7.32-7.23(\mathrm{~m}, 2 \mathrm{H}), 6.91-6.85(\mathrm{~m}, 2 \mathrm{H}), 6.28(\mathrm{~d}, J=1.1 \mathrm{~Hz}$, $1 \mathrm{H}), 6.16(\mathrm{~d}, J=1.1 \mathrm{~Hz}, 1 \mathrm{H}), 3.89(\mathrm{~d}, J=5.4 \mathrm{~Hz}, 3 \mathrm{H})$. Analytic data is in accordance with literature data. ${ }^{25}$ 


\section{(d) Procedure for synthesis of benzyl 2-mercaptoacetate (1B):}<smiles>O=C(CS)OCc1ccccc1</smiles>

To a round bottomed flask with $\mathrm{BnOH}(2.06 \mathrm{~mL}, 20 \mathrm{mmol})$ and 2-mercaptoacetic acid (1.66 $\mathrm{mL}, 24 \mathrm{mmol})$ in DCM $(120 \mathrm{~mL})$ was added $p$-Toluenesulfonic acid (0.3 equiv, $1.03 \mathrm{~g}, 6$ $\mathrm{mmol})$. The reaction mixture was stirred for 5 hours. The mixture was washed with saturated sodium bicarbonate solution $(150 \mathrm{~mL})$, brine $(200 \mathrm{~mL})$, dried over $\mathrm{Na}_{2} \mathrm{SO}_{4}$, concentrated and purified by flash chromatography (petroleum ether: ethyl acetate $=20: 1$ ) to give the benzyl 2-mercaptoacetate as colorless oil $(2.29 \mathrm{~g}, 63 \%)$.

${ }^{1} \mathrm{H}$ NMR $\left(400 \mathrm{MHz}, \mathrm{CDCl}_{3}\right) \delta 7.45-7.30(\mathrm{~m}, 5 \mathrm{H}), 5.18(\mathrm{~s}, 2 \mathrm{H}), 3.30(\mathrm{~d}, J=8.3 \mathrm{~Hz}, 2 \mathrm{H})$, $2.03(\mathrm{t}, J=8.3 \mathrm{~Hz}, 1 \mathrm{H})$. Analytic data is in accordance with literature data. ${ }^{33}$

\section{(e) General procedure $D$ for thiol compounds}

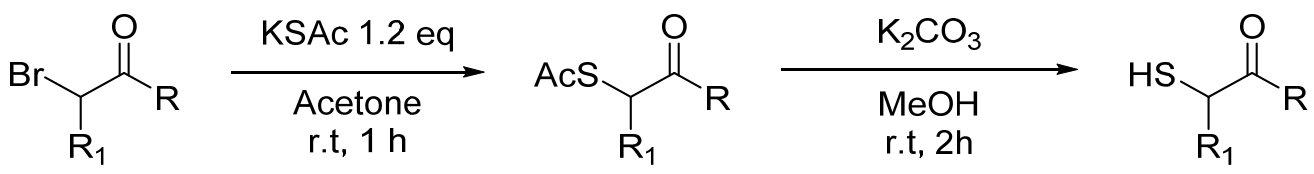

To a starting bromide $(2.0 \mathrm{mmol})$ in acetone $(20 \mathrm{~mL})$ was added potassium thioacetate (KSAc, 1.2 equiv) at room temperature. The reaction mixture was filtered after 1hours and the solvent was removed in vacuo. The resulting residue was distributed between water (20 $\mathrm{mL})$ and $\mathrm{CH}_{2} \mathrm{Cl}_{2}(30 \mathrm{~mL})$, and the combined organic layer was washed with brine, dried over $\mathrm{Na}_{2} \mathrm{SO}_{4}$, and concentrated in vacuo to give the thioacetate. To the round bottomed flask with $\mathrm{K}_{2} \mathrm{CO}_{3}(2.0 \mathrm{mmol})$ in $\mathrm{MeOH}(15 \mathrm{~mL})$ under $\mathrm{N}_{2}$ was added thioesters $(1.8 \mathrm{mmol})$ by syringe. The reaction mixture was stirred 1 hour before it was quenched with $\mathrm{HCl}(5 \mathrm{~mL}, 1$ $\mathrm{M})$. The mixture was extracted with $\mathrm{CH}_{2} \mathrm{Cl}_{2}(30 \mathrm{~mL})$, and the combined organic layer was washed with brine, dried over anhydrous $\mathrm{Na}_{2} \mathrm{SO}_{4}$ and concentrated in vacuo. The residue was used directly for the next step. 


\section{2-mercapto-1-phenylethan-1-one (1C)}<smiles>O=C(CS)c1ccccc1</smiles>

prepared from 2-bromo-1-phenylethan-1-one according to the general procedures as yellow oil (60\%), which was purified by flash chromatography (petroleum ether: ethyl acetate $=40$ : 1). ${ }^{1} \mathrm{H} \mathrm{NMR}\left(400 \mathrm{MHz}, \mathrm{CDCl}_{3}\right) \delta 8.00-7.93(\mathrm{~m}, 2 \mathrm{H}), 7.62-7.54(\mathrm{~m}, 1 \mathrm{H}), 7.53-7.46(\mathrm{~m}$, $2 \mathrm{H}), 3.96(\mathrm{~d}, J=7.3 \mathrm{~Hz}, 2 \mathrm{H}), 2.13(\mathrm{t}, J=7.3 \mathrm{~Hz}, 1 \mathrm{H})$. Analytic data is in accordance with literature data. ${ }^{34}$

2-mercapto-1-(p-tolyl) ethan-1-one (1D)<smiles>Cc1ccc(C(=O)CS)cc1</smiles>

Prepared from 2-bromo-1-(p-tolyl)ethan-1-one according to the general procedures as yellow oil (50\%), which was purified by flash chromatography (petroleum ether: ethyl acetate $=40: 1) .{ }^{1} \mathrm{H} \mathrm{NMR}\left(400 \mathrm{MHz}, \mathrm{CDCl}_{3}\right) \delta 7.86(\mathrm{~d}, J=8.3 \mathrm{~Hz}, 2 \mathrm{H}), 7.28(\mathrm{~d}, J=7.9 \mathrm{~Hz}$, $2 \mathrm{H}), 3.94(\mathrm{~d}, J=7.3 \mathrm{~Hz}, 2 \mathrm{H}), 2.42(\mathrm{~s}, 3 \mathrm{H}), 2.13(\mathrm{~d}, J=14.7 \mathrm{~Hz}, 1 \mathrm{H})$. Analytic data is in accordance with literature data. ${ }^{35}$

2-mercapto-1-(4-methoxyphenyl) ethan-1-one (1E)<smiles>COc1ccc(C(=O)CS)cc1</smiles>

prepared from 2-bromo-1-(4-methoxyphenyl)ethan-1-one according to the general procedures as yellow oil (65\%), which was purified by flash chromatography (petroleum ether: ethyl acetate $=10: 1) .{ }^{1} \mathrm{H} \mathrm{NMR}\left(400 \mathrm{MHz}, \mathrm{CDCl}_{3}\right) \delta 7.95(\mathrm{~d}, J=9.0 \mathrm{~Hz}, 2 \mathrm{H}), 6.96(\mathrm{~d}$, $J=8.9 \mathrm{~Hz}, 2 \mathrm{H}), 3.91(\mathrm{~d}, J=7.3 \mathrm{~Hz}, 2 \mathrm{H}), 3.88(\mathrm{~s}, 3 \mathrm{H}), 2.14(\mathrm{t}, J=7.3 \mathrm{~Hz}, 1 \mathrm{H})$. Analytic data is in accordance with literature data. ${ }^{36}$ 


\section{(f) General procedure $\mathrm{E}$ for 1,4-dicarbonyl compounds}

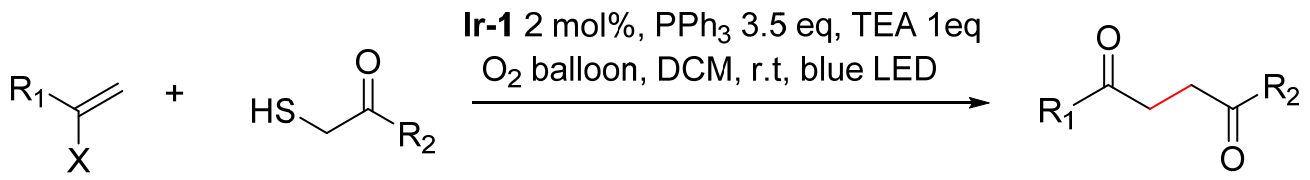

To the solution of alkenyl halogen $(0.5 \mathrm{mmol})$ and thiol $(0.75 \mathrm{mmol}, 1.5$ equiv $)$ in DCM $(10 \mathrm{~mL})$ were added $\operatorname{Ir}\left(\mathrm{dF}\left(\mathrm{CF}_{3}\right) \mathrm{ppy}_{2}(\mathrm{dtbbpy}) \mathrm{PF}_{6}(10 \mathrm{mg}, 1 \% \mathrm{mmol}, 0.02\right.$ equiv $), \mathrm{PPh}_{3}$ ( $1.75 \mathrm{mmol}, 3.5$ equiv), and triethylamine (TEA, $0.5 \mathrm{mmol}, 1.0$ equiv). The flask was quickly degassed three times and flushed with oxygen through balloon. The mixture was stirred at room temperature and irradiated with three $45 \mathrm{~W}$ blue LEDs $(5 \mathrm{~cm}$ away) for 7 hours. The reaction progress was monitored by TLC analysis. The solvent was removed in vacuo and the residue was extracted with ethyl acetate for three times. The combined organic layer was dried over anhydrous $\mathrm{Na}_{2} \mathrm{SO}_{4}$, and concentrated in vacuo. The crude product was purified by silica gel chromatography with EA: PE (1:30 - 1:10).

ethyl 4-oxo-4-phenylbutanoate (3a/3A)<smiles>CCOC(=O)CCC(=O)c1ccccc1</smiles>

(a) Prepared following the general procedure E employing (1-chlorovinyl) benzene (69 $\mathrm{mg}$, $0.5 \mathrm{mmol}, 1.0$ equiv) and ethyl 2 -mercaptoacetate $(90 \mathrm{mg}, 0.75 \mathrm{mmol}, 1.5$ equiv) as substrates. The desired product yielded $88 \%$ (determined by ${ }^{1} \mathrm{H}$ NMR).

(b) Prepared following the general procedure E employing (1-bromovinyl) benzene (90.9mg, $0.5 \mathrm{mmol}, 1.0$ equiv.) and ethyl 2 -mercaptoacetate $(90 \mathrm{mg}, 0.75 \mathrm{mmol}, 1.5$ equiv.) as substrates. The desired product yielded $94 \%$ (determined by ${ }^{1} \mathrm{H}$ NMR).

(c) Prepared following the general procedure E employing (1-iodovinyl) benzene (115 mg, $0.5 \mathrm{mmol}, 1.0$ equiv.), and ethyl 2 -mercaptoacetate ( $90 \mathrm{mg}, 0.75 \mathrm{mmol}, 1.5$ equiv.) as substrates. The desired product yielded $99 \%$ (determined by ${ }^{1} \mathrm{H}$ NMR).

Colorless oil. ${ }^{1} \mathrm{H}$ NMR $\left(400 \mathrm{MHz}, \mathrm{CDCl}_{3}\right) \delta 8.01-7.94(\mathrm{~m}, 2 \mathrm{H}), 7.56(\mathrm{t}, J=7.4 \mathrm{~Hz}, 1 \mathrm{H})$, $7.45(\mathrm{t}, J=7.7 \mathrm{~Hz}, 2 \mathrm{H}), 4.16(\mathrm{q}, J=7.1 \mathrm{~Hz}, 2 \mathrm{H}), 3.30(\mathrm{t}, J=6.7 \mathrm{~Hz}, 2 \mathrm{H}), 2.74(\mathrm{t}, J=6.6 \mathrm{~Hz}$, $2 \mathrm{H}), 1.25(\mathrm{t}, J=7.1 \mathrm{~Hz}, 3 \mathrm{H}) .{ }^{13} \mathrm{C}$ NMR $\left(101 \mathrm{MHz}, \mathrm{CDCl}_{3}\right) \delta 198.2,172.9,136.6,133.2$, 128.6, 128.1, 60.7, 33.4, 28.3, 14.2. HRMS (ESI) calcd for $\mathrm{C}_{12} \mathrm{H}_{14} \mathrm{NaO}_{3}[\mathrm{M}+\mathrm{Na}]^{+} m / z=$ 229.0835, found: 229.0832 . Analytic data is in accordance with literature data. ${ }^{37}$ 
ethyl 4-(naphthalen-2-yl)-4-oxobutanoate (3b)<smiles>CCOC(=O)CCC(=O)c1ccc2ccccc2c1</smiles>

(a) Prepared following the general procedure E employing 2-(1-chlorovinyl) -naphthalene (94 mg, $0.5 \mathrm{mmol}, 1.0$ equiv.) and ethyl 2-mercaptoacetate ( $90 \mathrm{mg}, 0.75 \mathrm{mmol}, 1.5$ equiv.) as substrates. The desired product was obtained $(115 \mathrm{mg}$, yield $90 \%)$ after purification by flash column chromatography on silica gel (petroleum ether $/$ ethyl acetate $=30 / 1-20 / 1$ )

(b) Prepared following the general procedure E employing 2-(1-bromovinyl) -naphthalene ( $116 \mathrm{mg}, 0.5 \mathrm{mmol}, 1.0$ equiv.) and ethyl 2-mercaptoacetate ( $90 \mathrm{mg}, 0.75 \mathrm{mmol}, 1.5$ equiv.) as substrates. The desired product was obtained $(121 \mathrm{mg}$, yield $95 \%)$ after purification by flash column chromatography on silica gel (petroleum ether/ ethyl acetate $=30 / 1-20 / 1$ )

(c) Prepared following the general procedure E employing 2-(1-iodovinyl) naphthalene (140mg, 0.5mmol, 1.0 equiv.) and ethyl 2-mercaptoacetate $(90 \mathrm{mg}, 0.75 \mathrm{mmol}, 1.5$ equiv.) as substrates. The desired product was obtained $(121 \mathrm{mg}$, yield $95 \%)$ after purification by flash column chromatography on silica gel (petroleum ether ethyl acetate $=30 / 1-20 / 1$ ) Brown oil. ${ }^{1} \mathrm{H}$ NMR $\left(400 \mathrm{MHz}, \mathrm{CDCl}_{3}\right) \delta 8.52(\mathrm{~s}, 1 \mathrm{H}), 8.05(\mathrm{dd}, J=8.6,1.6 \mathrm{~Hz}, 1 \mathrm{H}), 7.97$ $(\mathrm{d}, J=7.9 \mathrm{~Hz}, 1 \mathrm{H}), 7.89(\mathrm{t}, J=8.4 \mathrm{~Hz}, 2 \mathrm{H}), 7.58(\mathrm{ddd}, J=15.0,13.6,6.8 \mathrm{~Hz}, 2 \mathrm{H}), 4.22-$ $4.11(\mathrm{~m}, 2 \mathrm{H}), 3.46(\mathrm{t}, J=6.7 \mathrm{~Hz}, 2 \mathrm{H}), 2.82(\mathrm{t}, J=6.7 \mathrm{~Hz}, 2 \mathrm{H}), 1.28(\mathrm{t}, J=7.1 \mathrm{~Hz}, 3 \mathrm{H}) .{ }^{13} \mathrm{C}$ NMR (101 MHz, $\left.\mathrm{CDCl}_{3}\right) \delta 198.1,173.0,135.7,133.9,132.5,129.8,129.6,128.5,128.4$, 127.8, 126.8, 123.8, 60.7, 33.5, 28.4, 14.2. HRMS (ESI) calcd for $\mathrm{C}_{16} \mathrm{H}_{16} \mathrm{NaO}_{3}[\mathrm{M}+\mathrm{Na}]^{+}$ $\mathrm{m} / \mathrm{z}=279.0992$, found: 279.0992 . Analytic data is in accordance with literature data. ${ }^{38}$

ethyl 4-(4-fluorophenyl)-4-oxobutanoate (3c)<smiles>CCOC(=O)CCC(=O)c1ccc(F)cc1</smiles>

(a) Prepared following the general procedure E employing 1-(1-chlorovinyl) -4-fluorobenzene (78mg, 0.5mmol, 1.0 equiv.), and ethyl 2-mercaptoacetate $(90 \mathrm{mg}, 0.75$ mmol, 1.5 equiv.) as substrates. The desired product yielded $86 \%$ (determined by ${ }^{1} \mathrm{H}$ NMR).

(b) Prepared following the general procedure E employing 1-(1-bromovinyl) -4-fluorobenzene (100mg, 0.5mmol, 1.0 equiv.) and ethyl 2 -mercaptoacetate $(90 \mathrm{mg}, 0.75$ mmol, 1.5 equiv.) as substrates. The desired product yielded $96 \%$ (determined by ${ }^{1} \mathrm{H}$ NMR).

(c). Prepared following the general procedure E employing 1-fluoro-4-(1-Iodovinyl) -benzene (124mg, 0.5mmol, 1.0 equiv.) and ethyl 2-mercaptoacetate ( $90 \mathrm{mg}, 0.75 \mathrm{mmol}, 1.5$ equiv.) as substrates. The desired product yielded $92 \%$ (determined by ${ }^{1} \mathrm{H} \mathrm{NMR}$ ).

Colorless oil. ${ }^{1} \mathrm{H}$ NMR $\left(400 \mathrm{MHz}, \mathrm{CDCl}_{3}\right) \delta 8.00(\mathrm{dd}, J=8.8,5.4 \mathrm{~Hz}, 2 \mathrm{H}), 7.12(\mathrm{t}, J=8.6$ $\mathrm{Hz}, 2 \mathrm{H}), 4.14(\mathrm{q}, J=7.2 \mathrm{~Hz}, 2 \mathrm{H}), 3.26(\mathrm{t}, J=6.6 \mathrm{~Hz}, 2 \mathrm{H}), 2.74(\mathrm{t}, J=6.6 \mathrm{~Hz}, 2 \mathrm{H}), 1.25(\mathrm{t}, J$ $=7.1 \mathrm{~Hz}, 3 \mathrm{H}) .{ }^{13} \mathrm{C} \mathrm{NMR}\left(101 \mathrm{MHz}, \mathrm{CDCl}_{3}\right) \delta 196.6,172.8,165.8\left({ }^{1} J_{\mathrm{CF}}=255.5 \mathrm{~Hz}\right), 133.1$ 
$\left({ }^{4} J_{\mathrm{CF}}=3.0 \mathrm{~Hz}\right), 130.7\left({ }^{3} J_{\mathrm{CF}}=9.1 \mathrm{~Hz}\right), 115.7\left({ }^{2} J_{\mathrm{CF}}=22.2 \mathrm{~Hz}\right), 60.7,33.3,28.3$, 14.2. HRMS (ESI) calcd for $\mathrm{C}_{12} \mathrm{H}_{13} \mathrm{FNaO}_{3}[\mathrm{M}+\mathrm{Na}]^{+} \mathrm{m} / \mathrm{z}=247.0741$, found: 247.0741 . Analytic data is in accordance with literature data. ${ }^{37} \#$

ethyl 4-(4-chlorophenyl)-4-oxobutanoate (3d)<smiles>CCOC(=O)CCC(=O)c1ccc(Cl)cc1</smiles>

(a) Prepared following the general procedure E employing 1-chloro-4-(1-chloro-vinyl)benzene ( $85 \mathrm{mg}, 0.5 \mathrm{mmol}, 1.0$ equiv.), and ethyl 2-mercaptoacetate $(90 \mathrm{mg}, 0.75$ mmol, 1.5 equiv.) as substrates. The desired product yielded $82 \%$ (determined by ${ }^{1} \mathrm{H}$ NMR).

(b) Prepared following the general procedure E employing 1-chloro-4-(1-bromo-vinyl) -benzene (107mg, 0.5mmol, 1.0 equiv.), and ethyl 2-mercaptoacetate ( $90 \mathrm{mg}, 0.75 \mathrm{mmol}$, 1.5 equiv.) as substrates. The desired product yielded $99 \%$ (determined by ${ }^{1} \mathrm{H} \mathrm{NMR}$ ).

(c) Prepared following the general procedure E employing 1-chloro-4-(1-iodovinyl) -benzene (132mg, 0.5mmol, 1.0 equiv.) and ethyl 2-mercaptoacetate $(90 \mathrm{mg}, 0.75 \mathrm{mmol}, 1.5$ equiv.) as substrates. The desired product yielded $99 \%$ (determined by ${ }^{1} \mathrm{H} \mathrm{NMR}$ ).

Colorless oil, ${ }^{1} \mathrm{H}$ NMR $\left(400 \mathrm{MHz}, \mathrm{CDCl}_{3}\right) \delta 7.93(\mathrm{~d}, J=8.5 \mathrm{~Hz}, 2 \mathrm{H}), 7.45(\mathrm{~d}, J=8.5 \mathrm{~Hz}$, $2 \mathrm{H}), 4.16(\mathrm{q}, J=7.1 \mathrm{~Hz}, 2 \mathrm{H}), 3.28(\mathrm{t}, J=6.6 \mathrm{~Hz}, 2 \mathrm{H}), 2.76(\mathrm{t}, J=6.6 \mathrm{~Hz}, 2 \mathrm{H}), 1.28(\mathrm{~d}, J=$ $7.1 \mathrm{~Hz}, 3 \mathrm{H}) .{ }^{13} \mathrm{C} \mathrm{NMR}\left(101 \mathrm{MHz}, \mathrm{CDCl}_{3}\right) \delta 197.0,172.8,139.7,134.9,129.5,129.0,60.8$, 33.4, 28.2, 14.2. HRMS (ESI) calcd for $\mathrm{C}_{12} \mathrm{H}_{13} \mathrm{ClNaO}_{3}[\mathrm{M}+\mathrm{Na}]^{+} \mathrm{m} / \mathrm{z}=263.0445$, found: 263.0445. Analytic data is in accordance with literature data. ${ }^{37}$

ethyl 4-(4-bromophenyl)-4-oxobutanoate (3e)<smiles>CCOC(=O)CCC(=O)c1ccc(Br)cc1</smiles>

(a) Prepared following the general procedure E employing 1-bromo-4- -(1-chloro-vinyl) -benzene (108mg, $0.5 \mathrm{mmol}, 1.0$ equiv.) and ethyl 2-mercaptoacetate $(90 \mathrm{mg}, 0.75 \mathrm{mmol}, 1.5$ equiv.) as substrates. The desired product was obtained (132 mg, yield 93\%) after purification by flash column chromatography on silica gel (petroleum ether/ ethyl acetate $=$ $30 / 1-20 / 1)$

(b) Prepared following the general procedure E employing 1-bromo-4- -(1-bromovinyl) -benzene (130mg, 0.5mmol, 1.0 equiv.) and ethyl 2-mercaptoacetate $(90 \mathrm{mg}, 0.75 \mathrm{mmol}, 1.5$ equiv.) as substrates. The desired product was obtained (128 mg, yield 90\%) after purification by flash column chromatography on silica gel (petroleum ether/ ethyl acetate $=$ $30 / 1-20 / 1)$

(c) Prepared following the general procedure E employing 1-bromo-4- -(1-iodovinyl) -benzene (154mg, $0.5 \mathrm{mmol}, 1.0$ equiv.) and ethyl 2-mercaptoacetate $(90 \mathrm{mg}, 0.75 \mathrm{mmol}, 1.5$ equiv.) as substrates. The desired product was obtained (135 mg, yield 95\%) after purification by flash column chromatography on silica gel (petroleum ether/ ethyl acetate $=$ 
$30 / 1-20 / 1)$

Colorless oil. ${ }^{1} \mathrm{H}$ NMR $\left(400 \mathrm{MHz}, \mathrm{CDCl}_{3}\right) \delta 7.85(\mathrm{~d}, J=8.6 \mathrm{~Hz}, 2 \mathrm{H}), 7.61(\mathrm{~d}, J=8.6 \mathrm{~Hz}$, $2 \mathrm{H}), 4.16(\mathrm{q}, J=7.1 \mathrm{~Hz}, 2 \mathrm{H}), 3.27(\mathrm{t}, J=6.6 \mathrm{~Hz}, 2 \mathrm{H}), 2.75(\mathrm{t}, J=6.6 \mathrm{~Hz}, 2 \mathrm{H}), 1.27(\mathrm{t}, J=$ $7.1 \mathrm{~Hz}, 3 \mathrm{H}) .{ }^{13} \mathrm{C} \mathrm{NMR}\left(101 \mathrm{MHz}, \mathrm{CDCl}_{3}\right) \delta 197.2,172.8,135.3,132.0,129.6,128.4,60.8$, 33.3, 28.2, 14.2. HRMS (ESI) calcd for $\mathrm{C}_{12} \mathrm{H}_{13} \mathrm{BrNaO}_{3}[\mathrm{M}+\mathrm{Na}]^{+} \mathrm{m} / \mathrm{z}=306.9940$, found: 306.9940. Analytic data is in accordance with literature data. ${ }^{37}$

ethyl 4-oxo-4-(p-tolyl) butanoate (3f)<smiles>CCOC(=O)CCC(=O)c1ccc(C)cc1</smiles>

(a) Prepared following the general procedure E employing 1-(1-chlorovinyl)-4 -methylbenzene ( $76 \mathrm{mg}, 0.5 \mathrm{mmol}, 1.0$ equiv.) and ethyl 2-mercaptoacetate $(90 \mathrm{mg}, 0.75$ mmol, 1.5 equiv.) as substrates. The desired product yielded $78 \%$ (determined by ${ }^{1} \mathrm{H}$ NMR).

(b) Prepared following the general procedure E employing 1-(1-bromovinyl)-4 -methylbenzene ( $98 \mathrm{mg}, 0.5 \mathrm{mmol}, 1.0$ equiv.) and ethyl 2-mercaptoacetate $(90 \mathrm{mg}, 0.75$ mmol, 1.5 equiv.) as substrates. The desired product yielded $80 \%$ (determined by ${ }^{1} \mathrm{H}$ NMR).

(c) Prepared following the general procedure E employing 1-(1-iodovinyl)-4 -methylbenzene ( $122 \mathrm{mg}, 0.5 \mathrm{mmol}, 1.0$ equiv.) and ethyl2-mercaptoacetate $(90 \mathrm{mg}, 0.75$ mmol, 1.5 equiv.) as substrates. The desired product yielded $98 \%$ (determined by ${ }^{1} \mathrm{H}$ NMR).

White solid. ${ }^{1} \mathrm{H}$ NMR $\left(400 \mathrm{MHz}, \mathrm{CDCl}_{3}\right) \delta 7.88(\mathrm{~d}, J=8.2 \mathrm{~Hz}, 2 \mathrm{H}), 7.26(\mathrm{~d}, J=8.1 \mathrm{~Hz}, 2 \mathrm{H})$, $4.16(\mathrm{q}, J=7.1 \mathrm{~Hz}, 2 \mathrm{H}), 3.29(\mathrm{t}, J=6.7 \mathrm{~Hz}, 2 \mathrm{H}), 2.74(\mathrm{t}, J=6.7 \mathrm{~Hz}, 2 \mathrm{H}), 2.41(\mathrm{~s}, 3 \mathrm{H}), 1.26$ $(\mathrm{t}, J=7.1 \mathrm{~Hz}, 3 \mathrm{H}) \cdot{ }^{13} \mathrm{C} \mathrm{NMR}\left(101 \mathrm{MHz}, \mathrm{CDCl}_{3}\right) \delta 197.8,173.0,144.0,134.2,129.3,128.2$, 60.6, 33.3, 28.4, 21.7, 14.2. HRMS (ESI) calcd for $\mathrm{C}_{13} \mathrm{H}_{16} \mathrm{NaO}_{3}[\mathrm{M}+\mathrm{Na}]^{+} \mathrm{m} / \mathrm{z}=243.0992$, found: 243.0998. Analytic data is in accordance with literature data. ${ }^{39}$ 
ethyl 4-(4-ethylphenyl)-4-oxobutanoate (3g)<smiles>CCOC(=O)CCC(=O)c1ccc(CC)cc1</smiles>

(a) Prepared following the general procedure E employing 1-(1-chlorovinyl)-4-ethyl benzene (83mg, 0.5mmol, 1.0 equiv.) and ethyl 2-mercaptoacetate $(90 \mathrm{mg}, 0.75 \mathrm{mmol}, 1.5$ equiv.) as substrates. The desired product yielded $85 \%$ (determined by ${ }^{1} \mathrm{H}$ NMR).

(b) Prepared following the general procedure E employing 1-(1-bromovinyl)-4-ethyl benzene (105mg, $0.5 \mathrm{mmol}, 1.0$ equiv.) and ethyl 2-mercaptoacetate $(90 \mathrm{mg}, 0.75 \mathrm{mmol}, 1.5$ equiv.) as substrates. The desired product yielded $82 \%$ (determined by ${ }^{1} \mathrm{H}$ NMR).

(c) Prepared following the general procedure E employing 1-(1-iodovinyl)-4-ethyl benzene (129mg, 0.5mmol, 1.0 equiv.) and ethyl 2-mercaptoacetate ( $90 \mathrm{mg}, 0.75 \mathrm{mmol}, 1.5$ equiv.) as substrates. The desired product yielded $99 \%$ (determined by ${ }^{1} \mathrm{H}$ NMR).

Colorless oil. ${ }^{1} \mathrm{H}$ NMR $\left(400 \mathrm{MHz}, \mathrm{CDCl}_{3}\right) \delta 7.91(\mathrm{~d}, J=8.2 \mathrm{~Hz}, 2 \mathrm{H}), 7.29(\mathrm{~d}, J=8.1 \mathrm{~Hz}$, $2 \mathrm{H}), 4.16(\mathrm{q}, J=7.1 \mathrm{~Hz}, 2 \mathrm{H}), 3.30(\mathrm{t}, J=6.7 \mathrm{~Hz}, 2 \mathrm{H}), 2.75(\mathrm{t}, J=6.6 \mathrm{~Hz}, 2 \mathrm{H}), 2.71(\mathrm{dd}, J=$ $14.5,6.8 \mathrm{~Hz}, 2 \mathrm{H}), 1.26(\mathrm{tt}, J=7.4,3.2 \mathrm{~Hz}, 6 \mathrm{H}) .{ }^{13} \mathrm{C} \mathrm{NMR}\left(101 \mathrm{MHz}, \mathrm{CDCl}_{3}\right) \delta 197.8,173.0$, 150.2, 134.4, 128.3, 128.1, 60.6, 33.3, 29.0, 28.4, 15.2, 14.2. HRMS (ESI) calcd for $\mathrm{C}_{14} \mathrm{H}_{18} \mathrm{NaO}_{3}[\mathrm{M}+\mathrm{Na}]^{+} \mathrm{m} / \mathrm{z}=257.1148$, found: 257.1149 . Analytic data is in accordance with literature data. ${ }^{40}$

ethyl 4-oxo-4-(4-propylphenyl) butanoate (3h)<smiles>CCCc1ccc(C(=O)CCC(=O)OCC)cc1</smiles>

(a) Prepared following the general procedure E employing 1-(1-chlorovinyl) -4-propylbenzene (90mg, $0.5 \mathrm{mmol}, 1.0$ equiv.) and ethyl 2-mercaptoacetate $(90 \mathrm{mg}, 0.75$ mmol, 1.5 equiv.) as substrates. The desired product yielded $81 \%$ (determined by ${ }^{1} \mathrm{H}$ NMR).

(b) Prepared following the general procedure E employing 1-(1-bromovinyl) -4-propylbenzene ( $112 \mathrm{mg}, 0.5 \mathrm{mmol}, 1.0$ equiv.), and ethyl 2-mercaptoacetate ( $90 \mathrm{mg}, 0.75$ mmol, 1.5 equiv.) as substrates. The desired product yielded $86 \%$ (determined by ${ }^{1} \mathrm{H}$ NMR).

(c) Prepared following the general procedure $\mathrm{E}$ employing 1-(1-iodovinyl) -4-propylbenzene (136mg, $0.5 \mathrm{mmol}, 1.0$ equiv.) and ethyl 2-mercaptoacetate $(90 \mathrm{mg}, 0.75$ mmol, 1.5 equiv.) as substrates. The desired product yielded 99\% (determined by ${ }^{1} \mathrm{H}$ NMR).

Colorless oil. ${ }^{1} \mathrm{H}$ NMR $\left(400 \mathrm{MHz}, \mathrm{CDCl}_{3}\right) \delta 7.91(\mathrm{~d}, J=8.2 \mathrm{~Hz}, 2 \mathrm{H}), 7.26(\mathrm{~d}, J=8.1 \mathrm{~Hz}$, $2 \mathrm{H}), 4.16(\mathrm{q}, J=7.1 \mathrm{~Hz}, 2 \mathrm{H}), 3.29(\mathrm{t}, J=6.7 \mathrm{~Hz}, 2 \mathrm{H}), 2.75(\mathrm{t}, J=6.7 \mathrm{~Hz}, 2 \mathrm{H}), 2.69-2.57$ (m, $2 \mathrm{H}), 1.75-1.60(\mathrm{~m}, 3 \mathrm{H}), 1.27(\mathrm{t}, J=7.1 \mathrm{~Hz}, 3 \mathrm{H}), 0.94(\mathrm{t}, J=7.3 \mathrm{~Hz}, 3 \mathrm{H}) .{ }^{13} \mathrm{C}$ NMR $\left(101 \mathrm{MHz}, \mathrm{CDCl}_{3}\right) \delta 197.8,173.0,148.7,134.4,128.7,128.2,60.6,38.0,33.3,28.4,24.2$, 14.2, 13.7. HRMS (ESI) calcd for $\mathrm{C}_{15} \mathrm{H}_{20} \mathrm{NaO}_{3}[\mathrm{M}+\mathrm{Na}]^{+} \mathrm{m} / \mathrm{z}=271.1305$, found: 271.1304. 
Analytic data is in accordance with literature data. ${ }^{40}$

ethyl 4-(4-methoxyphenyl)-4-oxobutanoate (3i)<smiles>CCOC(=O)CCC(=O)c1ccc(OC)cc1</smiles>

(a) Prepared following the general procedure E employing 1-(1-chlorovinyl)-4 -methoxy benzene (84mg, $0.5 \mathrm{mmol}, 1.0$ equiv.) and ethyl 2-mercaptoacetate $(90 \mathrm{mg}, 0.75 \mathrm{mmol}, 1.5$ equiv.) as substrates. The desired product was obtained ( $95 \mathrm{mg}$, yield $80 \%)$ after purification by flash column chromatography on silica gel (petroleum ether/ ethyl acetate $=20 / 1$ 10/1).

(b) Prepared following the general procedure E employing 1-(1-bromovinyl)-4 -methoxy benzene (106mg, $0.5 \mathrm{mmol}, 1.0$ equiv.) and ethyl 2-mercaptoacetate $(90 \mathrm{mg}, 0.75 \mathrm{mmol}, 1.5$ equiv.) as substrates. The desired product was obtained (112 mg, yield 95\%) after purification by flash column chromatography on silica gel (petroleum ether/ ethyl acetate = 20/1 - 10/1).

(c) Prepared following the general procedure E employing 1-(1-iodovinyl)-4 methoxy benzene (130mg, $0.5 \mathrm{mmol}, 1.0$ equiv.) and ethyl 2-mercaptoacetate $(90 \mathrm{mg}, 0.75 \mathrm{mmol}, 1.5$ equiv.) as substrates. The desired product was obtained (106 mg, yield 90\%) after purification by flash column chromatography on silica gel (petroleum ether/ ethyl acetate $=$ 20/1 - 10/1).

Brown solid. ${ }^{1} \mathrm{H}$ NMR (400 MHz, $\left.\mathrm{CDCl}_{3}\right) \delta 7.97(\mathrm{~d}, J=8.9 \mathrm{~Hz}, 2 \mathrm{H}), 6.94(\mathrm{~d}, J=8.9 \mathrm{~Hz}$, $2 \mathrm{H}), 4.16(\mathrm{q}, J=7.1 \mathrm{~Hz}, 2 \mathrm{H}), 3.87(\mathrm{~s}, 3 \mathrm{H}), 3.27(\mathrm{t}, J=6.7 \mathrm{~Hz}, 2 \mathrm{H}), 2.74(\mathrm{t}, J=6.7 \mathrm{~Hz}, 2 \mathrm{H})$, $1.27(\mathrm{t}, J=7.2 \mathrm{~Hz}, 3 \mathrm{H}) ;{ }^{13} \mathrm{C} \mathrm{NMR}\left(101 \mathrm{MHz}, \mathrm{CDCl}_{3}\right) \delta 196.7,173.1,163.6,130.3,129.7$, 113.7, 60.6, 55.5, 33.0, 28.4, 14.2. HRMS (ESI) calcd for $\mathrm{C}_{13} \mathrm{H}_{16} \mathrm{NaO}_{4}[\mathrm{M}+\mathrm{Na}]^{+} \mathrm{m} / \mathrm{z}=$ 259.0941, found: 259.0941. Analytic data is in accordance with literature data. ${ }^{37}$

ethyl 4-([1,1'-biphenyl]-4-yl)-4-oxobutanoate (3j)<smiles>CCOC(=O)CCC(=O)c1ccc(-c2ccccc2)cc1</smiles>

(a) Prepared following the general procedure E employing 1-(1-chlorovinyl)-4 1,1'-biphenyl (102 mg, $0.5 \mathrm{mmol}, 1.0$ equiv.) and ethyl 2-mercaptoacetate ( $90 \mathrm{mg}, 0.75 \mathrm{mmol}, 1.5$ equiv.) as substrates. The desired product yielded $85 \%$ (determined by ${ }^{1} \mathrm{H}$ NMR).

(b) Prepared following the general procedure E employing 1-(1-bromovinyl)-4 1,1'-biphenyl (129 mg, $0.5 \mathrm{mmol}, 1.0$ equiv.) and ethyl 2-mercaptoacetate $(90 \mathrm{mg}, 0.75$ mmol, 1.5 equiv.) as substrates. The desired product yielded $97 \%$ (determined by ${ }^{1} \mathrm{H}$ NMR).

(c) Prepared following the general procedure E employing 1-(1-iodovinyl)-4 1,1'-biphenyl (153mg, 0.5mmol, 1.0 equiv.) and ethyl 2-mercaptoacetate ( $90 \mathrm{mg}, 0.75 \mathrm{mmol}, 1.5$ equiv.) as substrates. The desired product yielded $99 \%$ (determined by ${ }^{1} \mathrm{H}$ NMR).

White solid. ${ }^{1} \mathrm{H}$ NMR $\left(400 \mathrm{MHz}, \mathrm{CDCl}_{3}\right) \delta 8.05(\mathrm{~d}, J=5.3 \mathrm{~Hz}, 2 \mathrm{H}), 7.68(\mathrm{~d}, J=8.4 \mathrm{~Hz}, 2 \mathrm{H})$, 
$7.61(\mathrm{~d}, J=5.2 \mathrm{~Hz}, 2 \mathrm{H}), 7.42(\mathrm{~m}, J=29.1,6.7 \mathrm{~Hz}, 3 \mathrm{H}), 4.16(\mathrm{q}, J=7.1 \mathrm{~Hz}, 2 \mathrm{H}), 3.33(\mathrm{t}, J=$ $6.7 \mathrm{~Hz}, 2 \mathrm{H}), 2.77(\mathrm{t}, J=6.7 \mathrm{~Hz}, 2 \mathrm{H}), 1.26(\mathrm{t}, J=7.1 \mathrm{~Hz}, 3 \mathrm{H}) .{ }^{13} \mathrm{C} \mathrm{NMR}\left(101 \mathrm{MHz}, \mathrm{CDCl}_{3}\right)$ $\delta 197.7,172.9,145.9,139.9,132.3,132.2,131.5,128.9,128.6,128.4,128.2,127.3,60.7,33.4$, 28.3, 14.2. HRMS (ESI) calcd for $\mathrm{C}_{18} \mathrm{H}_{18} \mathrm{NaO}_{3}[\mathrm{M}+\mathrm{Na}]^{+} \mathrm{m} / \mathrm{z}=305.1148$, found: 305.1145. Analytic data is in accordance with literature data. ${ }^{41}$

ethyl 4-(3-fluorophenyl)-4-oxobutanoate (3k)<smiles>CCOC(=O)CCC(=O)c1cccc(F)c1</smiles>

(a) Prepared following the general procedure E employing 1-(1-chlorovinyl) -3-fluorobenzene $(78 \mathrm{mg}, 0.5 \mathrm{mmol}, 1.0$ equiv.) and ethyl 2-mercaptoacetate $(90 \mathrm{mg}, 0.75$ mmol, 1.5 equiv.) as substrates. The desired product yielded $80 \%$ (determined by ${ }^{1} \mathrm{H}$ NMR).

(b) Prepared following the general procedure E employing 1-(1-bromovinyl) -3-fluorobenzene ( $100 \mathrm{mg}, 0.5 \mathrm{mmol}, 1.0$ equiv.) and ethyl 2-mercaptoacetate ( $90 \mathrm{mg}, 0.75$ mmol, 1.5 equiv.) as substrates. The desired product yielded $85 \%$ (determined by ${ }^{1} \mathrm{H}$ NMR).

(c) Prepared following the general procedure E employing 1-(1-iodovinyl) -3-fluorobenzene (124mg, $0.5 \mathrm{mmol}, 1.0$ equiv.) and ethyl 2 -mercaptoacetate ( $90 \mathrm{mg}, 0.75 \mathrm{mmol}, 1.5$ equiv.) as substrates. The desired product yielded $92 \%$ (determined by ${ }^{1} \mathrm{H}$ NMR).

Brown oil. ${ }^{1} \mathrm{H}$ NMR $\left(400 \mathrm{MHz}, \mathrm{CDCl}_{3}\right) \delta 7.76(\mathrm{~d}, J=7.7 \mathrm{~Hz}, 1 \mathrm{H}), 7.68-7.62(\mathrm{~m}, 1 \mathrm{H})$, $7.44(\mathrm{td}, J=8.0,5.7 \mathrm{~Hz}, 1 \mathrm{H}), 7.27(\mathrm{dd}, J=8.7,2.2 \mathrm{~Hz}, 1 \mathrm{H}), 4.15(\mathrm{q}, J=7.1 \mathrm{~Hz}, 2 \mathrm{H}), 3.27(\mathrm{t}$, $J=6.6 \mathrm{~Hz}, 2 \mathrm{H}), 2.75(\mathrm{t}, J=6.6 \mathrm{~Hz}, 2 \mathrm{H}), 1.26(\mathrm{~d}, J=7.1 \mathrm{~Hz}, 3 \mathrm{H}) \cdot{ }^{13} \mathrm{C} \mathrm{NMR}(101 \mathrm{MHz}$, $\left.\mathrm{CDCl}_{3}\right) \delta 196.9(\mathrm{~d}, J=2.0 \mathrm{~Hz}), 172.7,162.9(\mathrm{~d}, J=248.5 \mathrm{~Hz}), 138.6(\mathrm{~d}, J=5 \mathrm{~Hz}), 130.3(\mathrm{~d}, J$ $=7.0 \mathrm{~Hz}), 123.8(\mathrm{~d}, J=3.0 \mathrm{~Hz}), 120.2(\mathrm{~d}, J=21.2 \mathrm{~Hz}), 114.8(\mathrm{~d}, J=22.0 \mathrm{~Hz}), 60.7,33.5,28.2$, 14.2. HRMS (ESI) calcd for $\mathrm{C}_{12} \mathrm{H}_{13} \mathrm{FNaO}_{3}[\mathrm{M}+\mathrm{Na}]^{+} \mathrm{m} / \mathrm{z}=247.0741$, found:247.0741. Analytic data is in accordance with literature data. ${ }^{37}$

ethyl 4-(3-chlorophenyl)-4-oxobutanoate (31)<smiles>CCOC(=O)CCC(=O)c1cccc(Cl)c1</smiles>

(a) Prepared following the general procedure E employing 1-(1-chlorovinyl) -3-chlorobenzene ( $86 \mathrm{mg}, 0.5 \mathrm{mmol}, 1.0$ equiv.) and ethyl 2 -mercaptoacetate $(90 \mathrm{mg}, 0.75$ mmol, 1.5 equiv.) as substrates. The desired product yielded $85 \%$ (determined by ${ }^{1} \mathrm{H}$ NMR).

(b) Prepared following the general procedure E employing 1-(1-bromovinyl) -3-chlorobenzene ( $108 \mathrm{mg}, 0.5 \mathrm{mmol}, 1.0$ equiv.) and ethyl 2-mercaptoacetate $(90 \mathrm{mg}, 0.75$ mmol, 1.5 equiv.) as substrates. The desired product yielded $81 \%$ (determined by ${ }^{1} \mathrm{H}$ 
NMR).

(c) Prepared following the general procedure E employing 1-(1-iodovinyl) -3-chlorobenzene (132mg, $0.5 \mathrm{mmol}, 1.0$ equiv.) and ethyl 2-mercaptoacetate $(90 \mathrm{mg}, 0.75$ mmol, 1.5 equiv.) as substrates. The desired product yielded $99 \%$ (determined by ${ }^{1} \mathrm{H}$ NMR).

Brown oil. ${ }^{1} \mathrm{H}$ NMR $\left(400 \mathrm{MHz}, \mathrm{CDCl}_{3}\right) \delta 7.94(\mathrm{~s}, 1 \mathrm{H}), 7.84(\mathrm{~d}, J=7.8 \mathrm{~Hz}, 1 \mathrm{H}), 7.57-7.50$ $(\mathrm{m}, 1 \mathrm{H}), 7.39(\mathrm{~d}, J=7.9 \mathrm{~Hz}, 1 \mathrm{H}), 4.14(\mathrm{q}, J=7.2 \mathrm{~Hz}, 2 \mathrm{H}), 3.26(\mathrm{t}, J=6.6 \mathrm{~Hz}, 2 \mathrm{H}), 2.74(\mathrm{t}, J$ $=6.5 \mathrm{~Hz}, 2 \mathrm{H}), 1.24(\mathrm{~d}, J=7.1 \mathrm{~Hz}, 3 \mathrm{H}) .{ }^{13} \mathrm{C} \mathrm{NMR}\left(101 \mathrm{MHz}, \mathrm{CDCl}_{3}\right) \delta$ 195.9, 171.7, 137.1, 134.0, 132.1, 129.0, 127.2, 125.1, 59.7, 32.5, 27.2, 13.2. HRMS (ESI) calcd forC ${ }_{12} \mathrm{H}_{13} \mathrm{ClNaO}_{3}[\mathrm{M}+\mathrm{Na}]^{+} \mathrm{m} / \mathrm{z}=263.0445$, found: 263.0445. Analytic data is in accordance with literature data. ${ }^{42}$

ethyl 4-(3-bromophenyl)-4-oxobutanoate (3m)<smiles>CCOC(=O)CCC(=O)c1cccc(Br)c1</smiles>

(a) Prepared following the general procedure E employing 1-(1-chlorovinyl) -3-bromo benzene (113 mg, $0.5 \mathrm{mmol}, 1.0$ equiv.) and ethyl 2-mercaptoacetate $(106 \mathrm{mg}, 0.75 \mathrm{mmol}$, 1.5 equiv.) as substrates. The desired product yielded $87 \%$ (determined by ${ }^{1} \mathrm{H} \mathrm{NMR}$ ).

(b) Prepared following the general procedure E employing 1-(1-bromovinyl) -3-bromo benzene ( $130 \mathrm{mg}, 0.5 \mathrm{mmol}, 1.0$ equiv.) and ethyl 2-mercaptoacetate $(90 \mathrm{mg}, 0.75 \mathrm{mmol}$, 1.5 equiv.) as substrates. The desired product yielded $96 \%$ (determined by ${ }^{1} \mathrm{H} \mathrm{NMR}$ ).

(c) Prepared following the general procedure $\mathrm{E}$ employing 1-(1-iodovinyl) -3-bromobenzene (154mg, 0.5mmol, 1.0 equiv.) and ethyl 2-mercaptoacetate ( $90 \mathrm{mg}, 0.75$ mmol, 1.5 equiv.) as substrates. The desired product yielded $95 \%$ (determined by ${ }^{1} \mathrm{H}$ NMR).

Brown oil. ${ }^{1} \mathrm{H}$ NMR $\left(400 \mathrm{MHz}, \mathrm{CDCl}_{3}\right) \delta 8.10(\mathrm{t}, J=1.6 \mathrm{~Hz}, 1 \mathrm{H}), 7.92-7.86(\mathrm{~m}, 1 \mathrm{H}), 7.71$ $-7.65(\mathrm{~m}, 1 \mathrm{H}), 7.34(\mathrm{t}, J=7.9 \mathrm{~Hz}, 1 \mathrm{H}), 4.15(\mathrm{q}, J=7.2 \mathrm{~Hz}, 2 \mathrm{H}), 3.26(\mathrm{t}, J=6.6 \mathrm{~Hz}, 2 \mathrm{H})$, $2.74(\mathrm{t}, J=6.6 \mathrm{~Hz}, 2 \mathrm{H}), 1.24(\mathrm{~d}, J=7.2 \mathrm{~Hz}, 3 \mathrm{H}) .{ }^{13} \mathrm{C} \mathrm{NMR}\left(101 \mathrm{MHz}, \mathrm{CDCl}_{3}\right) \delta 196.9$, $172.7,138.3,136.1,131.2,130.2,126.6,123.0,60.8,33.5,28.2,14.2$. HRMS (ESI) calcd for $\mathrm{C}_{12} \mathrm{H}_{13} \mathrm{BrNaO}_{3}[\mathrm{M}+\mathrm{Na}]^{+} \mathrm{m} / \mathrm{z}=306.9940$, found: 306.9939 . Analytic data is in accordance with literature data. ${ }^{43}$

ethyl 4-oxo-4-(m-tolyl) butanoate (3n)<smiles>CCOC(=O)CCC(=O)c1cccc(C)c1</smiles>

(a) Prepared following the general procedure E employing 1-(1-chlorovinyl) -3-methyl (76 $\mathrm{mg}, 0.5 \mathrm{mmol}, 1.0$ equiv.) and ethyl 2-mercaptoacetate $(90 \mathrm{mg}, 0.75 \mathrm{mmol}, 1.5$ equiv.) as substrates. The desired product yielded $78 \%$ (determined by ${ }^{1} \mathrm{H}$ NMR). 
(b) Prepared following the general procedure E employing 1-(1-bromovinyl) -3-methyl (98 $\mathrm{mg}, 0.5 \mathrm{mmol}, 1.0$ equiv.) and ethyl 2-mercaptoacetate $(90 \mathrm{mg}, 0.75 \mathrm{mmol}, 1.5$ equiv.) as substrates. The desired product yielded $83 \%$ (determined by ${ }^{1} \mathrm{H}$ NMR).

(c) Prepared following the general procedure E employing 1-(1-iodovinyl) -3-methyl (122mg, 0.5mmol, 1.0 equiv.) and ethyl 2-mercaptoacetate ( $90 \mathrm{mg}, 0.75 \mathrm{mmol}, 1.5$ equiv.) as substrates. The desired product yielded $99 \%$ (determined by ${ }^{1} \mathrm{H}$ NMR).

Brown oil. ${ }^{1} \mathrm{H}$ NMR $\left(400 \mathrm{MHz}, \mathrm{CDCl}_{3}\right) \delta 7.78(\mathrm{~d}, J=8.6 \mathrm{~Hz}, 2 \mathrm{H}), 7.41-7.32(\mathrm{~m}, 2 \mathrm{H})$, $4.17(\mathrm{q}, J=8.6 \mathrm{~Hz}, 2 \mathrm{H}), 3.30(\mathrm{t}, J=6.7 \mathrm{~Hz}, 2 \mathrm{H}), 2.75(\mathrm{t}, J=6.7 \mathrm{~Hz}, 2 \mathrm{H}), 2.41(\mathrm{~s}, 3 \mathrm{H}), 1.27$ $(\mathrm{t}, J=7.1 \mathrm{~Hz}, 3 \mathrm{H}) .{ }^{13} \mathrm{C} \mathrm{NMR}\left(101 \mathrm{MHz}, \mathrm{CDCl}_{3}\right) \delta 198.4,173.0,138.4,136.7,134.0,128.6$, $128.5,125.3,60.7,33.5,28.4,21.4,14.2$. HRMS (ESI) calcd for $\mathrm{C}_{13} \mathrm{H}_{16} \mathrm{NaO}_{3}[\mathrm{M}+\mathrm{Na}]^{+} \mathrm{m} / \mathrm{z}$ $=243.0992$, found: 243.0996 . Analytic data is in accordance with literature data. ${ }^{44}$

ethyl 4-(2-fluorophenyl)-4-oxobutanoate (3o)<smiles>CCOC(=O)CCC(=O)c1ccccc1F</smiles>

(a) Prepared following the general procedure $\mathrm{E}$ employing 1-(1-chlorovinyl) -2-fluorobenzene ( $78 \mathrm{mg}, 0.5 \mathrm{mmol}, 1.0$ equiv.) and ethyl 2-mercaptoacetate $(90 \mathrm{mg}, 0.75$ mmol, 1.5 equiv.) as substrates. The desired product yielded $87 \%$ (determined by ${ }^{1} \mathrm{H}$ NMR).

(b) Prepared following the general procedure E employing 1-(1-bromovinyl) -2-fluorobenzene ( $100 \mathrm{mg}, 0.5 \mathrm{mmol}, 1.0$ equiv.) and ethyl 2-mercaptoacetate ( $90 \mathrm{mg}, 0.75$ mmol, 1.5 equiv.) as substrates. The desired product yielded $92 \%$ (determined by ${ }^{1} \mathrm{H}$ NMR).

(c) Prepared following the general procedure E employing 1-(1-iodovinyl) -2-fluoro (124mg, 0.5mmol, 1.0 equiv.) and ethyl 2-mercaptoacetate ( $90 \mathrm{mg}, 0.75 \mathrm{mmol}, 1.5$ equiv.) as substrates. The desired product yielded $98 \%$ (determined by ${ }^{1} \mathrm{H}$ NMR).

Brown oil. ${ }^{1} \mathrm{H}$ NMR $\left(400 \mathrm{MHz}, \mathrm{CDCl}_{3}\right) \delta 7.89(\mathrm{~m}, J=7.7,1.7 \mathrm{~Hz}, 1 \mathrm{H}), 7.53(\mathrm{~m}, J=7.1,5.1$, $1.7 \mathrm{~Hz}, 1 \mathrm{H}), 7.23(\mathrm{~d}, J=15.2 \mathrm{~Hz}, 1 \mathrm{H}), 7.14(\mathrm{dd}, J=11.2,8.4 \mathrm{~Hz}, 1 \mathrm{H}), 4.16(\mathrm{q}, J=7.1 \mathrm{~Hz}$, $2 \mathrm{H}), 3.32(\mathrm{td}, J=6.5,3.2 \mathrm{~Hz}, 2 \mathrm{H}), 2.74(\mathrm{t}, J=6.5 \mathrm{~Hz}, 2 \mathrm{H}), 1.27(\mathrm{t}, J=7.1 \mathrm{~Hz}, 3 \mathrm{H}) .{ }^{13} \mathrm{C}$ NMR $\left(101 \mathrm{MHz}, \mathrm{CDCl}_{3}\right) \delta 196.4(\mathrm{~d}, J=4.0 \mathrm{~Hz}), 172.8,162.1$ (d, $\left.J=255.5 \mathrm{~Hz}\right), 134.7$ (d, $J=9.1 \mathrm{~Hz}), 130.63(\mathrm{~d}, J=2.0 \mathrm{~Hz}), 125.2(\mathrm{~d}, J=13.1 \mathrm{~Hz}), 124.4(\mathrm{~d}, J=4.0 \mathrm{~Hz}), 116.7$ (d, $J$ $=23.2 \mathrm{~Hz}$ ), 60.6, $38.2(\mathrm{~d}, J=8.0 \mathrm{~Hz}), 28.3(\mathrm{~d}, J=3.0 \mathrm{~Hz}$ ), 14.2. HRMS (ESI) calcd for $\mathrm{C}_{12} \mathrm{H}_{13} \mathrm{FNaO}_{3}[\mathrm{M}+\mathrm{Na}]^{+} \mathrm{m} / \mathrm{z}=247.0741$, found:247.0742. Analytic data is in accordance with literature data. ${ }^{42}$

ethyl 4-(2-chlorophenyl)-4-oxobutanoate (3p)<smiles>CCOC(=O)CCC(=O)c1ccccc1Cl</smiles>

(a) Prepared following the general procedure $\mathrm{E}$ employing 1-(1-chlorovinyl) -2-chlorobenzene ( $78 \mathrm{mg}, 0.5 \mathrm{mmol}, 1.0$ equiv.) and ethyl 2-mercaptoacetate (90 mg, 0.75 
mmol, 1.5 equiv.) as substrates. The desired product yielded $86 \%$ (determined by ${ }^{1} \mathrm{H}$ NMR).

(b) Prepared following the general procedure E employing 1-(1-bromovinyl) -2-chlorobenzene ( $100 \mathrm{mg}, 0.5 \mathrm{mmol}, 1.0$ equiv.) and ethyl 2-mercaptoacetate ( $90 \mathrm{mg}, 0.75$ mmol, 1.5 equiv.) as substrates. The desired product yielded $91 \%$ (determined by ${ }^{1} \mathrm{H}$ NMR).

(c) Prepared following the general procedure E employing 1-(1-iodovinyl) -2-chlorobenzene (124mg, $0.5 \mathrm{mmol}, 1.0$ equiv.) and ethyl 2 -mercaptoacetate $(90 \mathrm{mg}, 0.75$ mmol, 1.5 equiv.) as substrates. The desired product yielded $93 \%$ (determined by ${ }^{1} \mathrm{H}$ NMR).

Brown oil. ${ }^{1} \mathrm{H}$ NMR $\left(400 \mathrm{MHz}, \mathrm{CDCl}_{3}\right) \delta 7.55(\mathrm{dt}, J=11.0,5.2 \mathrm{~Hz}, 1 \mathrm{H}), 7.45-7.30(\mathrm{~m}$, $3 \mathrm{H}), 4.16(\mathrm{q}, J=7.2 \mathrm{~Hz}, 2 \mathrm{H}), 3.26(\mathrm{t}, J=6.6 \mathrm{~Hz}, 2 \mathrm{H}), 2.76(\mathrm{t}, J=6.6 \mathrm{~Hz}, 2 \mathrm{H}), 1.27(\mathrm{t}, J=$ $7.1 \mathrm{~Hz}, 3 \mathrm{H}) .{ }^{13} \mathrm{C} \mathrm{NMR}\left(101 \mathrm{MHz}, \mathrm{CDCl}_{3}\right) \delta 201.2,172.6,138.9,131.9,131.0,130.5,129.2$, 126.9, 60.7, 37.6, 28.6, 14.2. HRMS (ESI) calcd for $\mathrm{C}_{12} \mathrm{H}_{13} \mathrm{ClNaO}_{3}[\mathrm{M}+\mathrm{Na}]^{+} \mathrm{m} / \mathrm{z}=$ 263.0445, found: 263.0446 . Analytic data is in accordance with literature data. ${ }^{45}$

ethyl 4-(2-bromophenyl)-4-oxobutanoate (3q)<smiles>CCOC(=O)CCC(=O)c1ccccc1Br</smiles>

(a) Prepared following the general procedure $\mathrm{E}$ employing 1-(1-chlorovinyl) -2-bromobenzene ( $108 \mathrm{mg}, 0.5 \mathrm{mmol}, 1.0$ equiv.) and ethyl 2-mercaptoacetate $(90 \mathrm{mg}, 0.75$ mmol, 1.5 equiv.) as substrates. The desired product yielded $80 \%$ (determined by ${ }^{1} \mathrm{H}$ NMR).

(b) Prepared following the general procedure $\mathrm{E}$ employing 1-(1-bromovinyl) -2-bromobenzene ( $130 \mathrm{mg}, 0.5 \mathrm{mmol}, 1.0$ equiv.) and ethyl 2-mercaptoacetate $(90 \mathrm{mg}, 0.75$ mmol, 1.5 equiv.) as substrates. The desired product yielded $95 \%$ (determined by ${ }^{1} \mathrm{H}$ NMR).

(c) Prepared following the general procedure $\mathrm{E}$ employing 1-(1-iodovinyl) -2-bromobenzene (154mg, 0.5mmol, 1.0 equiv.) and ethyl 2-mercaptoacetate ( $90 \mathrm{mg}, 0.75$ mmol, 1.5 equiv.) as substrates. The desired product yielded $98 \%$ (determined by ${ }^{1} \mathrm{H}$ NMR).

Brown oil. ${ }^{1} \mathrm{H}$ NMR $\left(400 \mathrm{MHz}, \mathrm{CDCl}_{3}\right) \delta 7.61(\mathrm{dd}, J=8.0,0.9 \mathrm{~Hz}, 1 \mathrm{H}), 7.49$ (dd, $J=7.6$, $1.7 \mathrm{~Hz}, 1 \mathrm{H}), 7.38(\mathrm{td}, J=7.5,1.1 \mathrm{~Hz}, 1 \mathrm{H}), 7.32-7.27(\mathrm{~m}, 1 \mathrm{H}), 4.16(\mathrm{q}, J=7.1 \mathrm{~Hz}, 2 \mathrm{H})$, $3.23(\mathrm{t}, J=6.6 \mathrm{~Hz}, 2 \mathrm{H}), 2.77(\mathrm{t}, J=6.6 \mathrm{~Hz}, 2 \mathrm{H}), 1.26(\mathrm{~d}, J=6.5 \mathrm{~Hz}, 3 \mathrm{H}) .{ }^{13} \mathrm{C} \operatorname{NMR}(101$ $\left.\mathrm{MHz}, \mathrm{CDCl}_{3}\right) \delta 202.1,172.6,141.3,133.7,131.7,128.8,127.5,118.7,60.8,37.4,28.5,14.2$. HRMS (ESI) calcd for $\mathrm{C}_{12} \mathrm{H}_{13} \mathrm{BrNaO}_{3}[\mathrm{M}+\mathrm{Na}]^{+} \mathrm{m} / \mathrm{z}=306.9940$, found: 306.9940. Analytic data is in accordance with literature data. ${ }^{46}$

ethyl 4-(2-methoxyphenyl)-4-oxobutanoate (3r) 
<smiles>CCOC(=O)CCC(=O)c1ccccc1OC</smiles>

(a) Prepared following the general procedure E employing 1-(1-chlorovinyl) -2-methoxybenzene ( $84 \mathrm{mg}, 0.5 \mathrm{mmol}, 1.0$ equiv.) and ethyl 2-mercaptoacetate ( $90 \mathrm{mg}, 0.75$ mmol, 1.5 equiv.) as substrates. The desired product yielded $82 \%$ (determined by ${ }^{1} \mathrm{H}$ NMR).

(b) Prepared following the general procedure E employing 1-(1-bromovinyl) -2-methoxybenzene (106 mg, $0.5 \mathrm{mmol}, 1.0$ equiv.) and ethyl 2 -mercaptoacetate $(90 \mathrm{mg}$, $0.75 \mathrm{mmol}, 1.5$ equiv.) as substrates. The desired product yielded $94 \%$ (determined by ${ }^{1} \mathrm{H}$ NMR).

(c) Prepared following the general procedure E employing 1-(1-iodovinyl) -2-methoxybenzene (130mg, $0.5 \mathrm{mmol}, 1.0$ equiv.), and ethyl 2-mercaptoacetate $(90 \mathrm{mg}$, $0.75 \mathrm{mmol}, 1.5$ equiv.) as substrates. The desired product yielded $99 \%$ (determined by ${ }^{1} \mathrm{H}$ NMR).

Brown oil. ${ }^{1} \mathrm{H}$ NMR $\left(400 \mathrm{MHz}, \mathrm{CDCl}_{3}\right) \delta 7.74(\mathrm{~d}, J=7.5 \mathrm{~Hz}, 1 \mathrm{H}), 7.45(\mathrm{t}, J=7.5 \mathrm{~Hz}, 1 \mathrm{H})$, $6.97(\mathrm{dd}, J=16.1,8.3 \mathrm{~Hz}, 2 \mathrm{H}), 4.13(\mathrm{dd}, J=14.4,7.2 \mathrm{~Hz}, 2 \mathrm{H}), 3.90(\mathrm{~s}, 3 \mathrm{H}), 3.31(\mathrm{t}, J=6.6$ $\mathrm{Hz}, 2 \mathrm{H}), 2.68(\mathrm{t}, J=6.7 \mathrm{~Hz}, 2 \mathrm{H}), 1.27-1.23(\mathrm{~m}, 3 \mathrm{H}) .{ }^{13} \mathrm{C} \mathrm{NMR}\left(101 \mathrm{MHz}, \mathrm{CDCl}_{3}\right) \delta 200.1$, 173.3, 158.9, 133.7, 130.5, 127.6, 120.7, 111.6, 60.5, 38.8, 29.7, 28.8, 14.2.HRMS (ESI) calcd for $\mathrm{C}_{13} \mathrm{H}_{16} \mathrm{NaO}_{4}[\mathrm{M}+\mathrm{Na}]^{+} \mathrm{m} / \mathrm{z}=259.0941$, found: 259.0941 . Analytic data is in accordance with literature data. ${ }^{37}$

ethyl 4-oxo-4-(thiophen-3-yl) butanoate (3s)<smiles>CCOC(=O)CCC(=O)c1ccsc1</smiles>

(a) Prepared following the general procedure E employing 3-(1-chlorovinyl) -thiophene (72 $\mathrm{mg}, 0.5 \mathrm{mmol}, 1.0$ equiv.) and ethyl 2-mercaptoacetate ( $90 \mathrm{mg}, 0.75 \mathrm{mmol}, 1.5$ equiv.) as substrates. The desired product was obtained a $(85 \mathrm{mg}$, yield $80 \%)$ after purification by flash column chromatography on silica gel (petroleum ether $/$ ethyl acetate $=30 / 1-10 / 1$ ).

(b) Prepared following the general procedure E employing 3-(1-bromovinyl) -thiophene (94 mg, $0.5 \mathrm{mmol}, 1.0$ equiv.) and ethyl 2-mercaptoacetate ( $90 \mathrm{mg}, 0.75 \mathrm{mmol}, 1.5$ equiv.) as substrates. The desired product was obtained as colorless oil (98 mg, yield $92 \%)$ after purification by flash column chromatography on silica gel (petroleum ether/ ethyl acetate $=$ 30/1 - 10/1).

Brown oil. ${ }^{1} \mathrm{H}$ NMR $\left(400 \mathrm{MHz}, \mathrm{CDCl}_{3}\right) \delta 8.10(\mathrm{dd}, J=2.9,1.2 \mathrm{~Hz}, 1 \mathrm{H}), 7.56(\mathrm{dd}, J=5.1$, $1.2 \mathrm{~Hz}, 1 \mathrm{H}), 7.32(\mathrm{dd}, J=5.1,2.9 \mathrm{~Hz}, 1 \mathrm{H}), 4.16(\mathrm{q}, J=7.1 \mathrm{~Hz}, 2 \mathrm{H}), 3.23(\mathrm{t}, J=6.7 \mathrm{~Hz}, 2 \mathrm{H})$, $2.74(\mathrm{t}, J=6.7 \mathrm{~Hz}, 2 \mathrm{H}), 1.27(\mathrm{t}, J=7.1 \mathrm{~Hz}, 3 \mathrm{H}) .{ }^{13} \mathrm{C} \mathrm{NMR}\left(101 \mathrm{MHz}, \mathrm{CDCl}_{3}\right) \delta 192.5$, $172.9,141.9,132.0,126.9,126.4,60.7,34.5,28.2,14.2$. HRMS (ESI) calcd for $\mathrm{C}_{10} \mathrm{H}_{12} \mathrm{SNaO}_{3}$ $[\mathrm{M}+\mathrm{Na}]^{+} \mathrm{m} / \mathrm{z}=235.0399$, found: 235.0395 . Analytical data is in accordance with literature data. ${ }^{47}$ 
benzyl 4-oxo-4-phenylbutanoate (3B)<smiles>O=C(CCC(=O)c1ccccc1)OCc1ccccc1</smiles>

Prepared following the general procedure E employing (1-bromovinyl) benzene ( $96 \mathrm{mg}, 0.5$ mmol, 1.0 equiv.) and benzyl 2-mercaptoacetate (137mg, $0.75 \mathrm{mmol}, 1.5$ equiv.) as substrates. The desired product was obtained as light-yellow oil (117 mg, yield $87 \%)$ after purification by flash column chromatography on silica gel (petroleum ether/ ethyl acetate $=$ 30/1 - 10/1). ${ }^{1} \mathrm{H}$ NMR $\left(400 \mathrm{MHz}, \mathrm{CDCl}_{3}\right) \delta 7.98(\mathrm{~d}, J=7.5 \mathrm{~Hz}, 2 \mathrm{H}), 7.57(\mathrm{t}, J=7.4 \mathrm{~Hz}$, $1 \mathrm{H}), 7.46(\mathrm{t}, J=7.7 \mathrm{~Hz}, 2 \mathrm{H}), 7.43-7.28(\mathrm{~m}, 5 \mathrm{H}), 5.15(\mathrm{~s}, 2 \mathrm{H}), 3.34(\mathrm{q}, J=6.5 \mathrm{~Hz}, 2 \mathrm{H})$, $2.83(\mathrm{t}, J=6.6 \mathrm{~Hz}, 2 \mathrm{H}) \cdot{ }^{13} \mathrm{C} \mathrm{NMR}\left(101 \mathrm{MHz}, \mathrm{CDCl}_{3}\right) \delta 198.0,172.8,136.6,135.9,133.3$, 128.6, 128.6, 128.2, 128.1, 66.5, 33.4, 28.3. HRMS (ESI) calcd for $\mathrm{C}_{17} \mathrm{H}_{16} \mathrm{NaO}_{3},[\mathrm{M}+\mathrm{Na}]^{+}$ $\mathrm{m} / \mathrm{z}=291.0992$, found: 291.0996 . Analytical data is in accordance with literature data. ${ }^{48}$

1,4-diphenylbutane-1,4-dione (3C)<smiles>O=C(CCC(=O)c1ccccc1)c1ccccc1</smiles>

Prepared following the general procedure E employing (1-bromovinyl) benzene ( $96 \mathrm{mg}, 0.5$ mmol, 1.0 equiv.) and 2-mercapto-1-phenyl ethan-1-one (114mg, $0.75 \mathrm{mmol}, 1.5$ equiv.) as substrates. The desired product was obtained as light-yellow solid (106 mg, yield $89 \%)$ after purification by flash column chromatography on silica gel (petroleum ether/ ethyl acetate = 30/1 - 10/1). ${ }^{1} \mathrm{H}$ NMR $\left(400 \mathrm{MHz}, \mathrm{CDCl}_{3}\right) \delta 8.03(\mathrm{~d}, J=7.8 \mathrm{~Hz}, 4 \mathrm{H}), 7.57(\mathrm{t}, J=7.4 \mathrm{~Hz}$, $2 \mathrm{H}), 7.47(\mathrm{t}, J=7.6 \mathrm{~Hz}, 4 \mathrm{H}), 3.46(\mathrm{~s}, 4 \mathrm{H}) .{ }^{13} \mathrm{C} \mathrm{NMR}\left(101 \mathrm{MHz}, \mathrm{CDCl}_{3}\right) \delta 198.7,136.8$, 133.2, 128.6, 128.2, 32.6. HRMS (ESI) calcd forC ${ }_{16} \mathrm{H}_{14} \mathrm{NaO}_{2},[\mathrm{M}+\mathrm{Na}]^{+} \mathrm{m} / \mathrm{z}=261.0886$, found: 261.0890 . Analytical data is in accordance with literature data. ${ }^{49}$ 
phenyl-4-(p-tolyl)butane-1,4-dione (3D)<smiles>Cc1ccc(C(=O)CCC(=O)c2ccccc2)cc1</smiles>

Prepared following the general procedure E employing (1-bromovinyl) benzene ( $96 \mathrm{mg}, 0.5$ mmol, 1.0 equiv.) and 2-mercapto-1-(p-tolyl) ethan-1-one (125 mg, $0.75 \mathrm{mmol}, 1.5$ equiv.) as substrates. The desired product was obtained as light-yellow solid (107 $\mathrm{mg}$, yield $85 \%$ ) after purification by flash column chromatography on silica gel (petroleum ether/ ethyl acetate $=30 / 1-10 / 1) .{ }^{1} \mathrm{H} \mathrm{NMR}\left(400 \mathrm{MHz}, \mathrm{CDCl}_{3}\right) \delta 7.97(\mathrm{~d}, J=7.4 \mathrm{~Hz}, 2 \mathrm{H}), 7.87(\mathrm{~d}, J=$ $8.0 \mathrm{~Hz}, 2 \mathrm{H}), 7.51(\mathrm{t}, J=7.3 \mathrm{~Hz}, 1 \mathrm{H}), 7.41(\mathrm{t}, J=7.5 \mathrm{~Hz}, 2 \mathrm{H}), 7.22(\mathrm{~s}, 2 \mathrm{H}), 3.38(\mathrm{~s}, 4 \mathrm{H})$, $2.35(\mathrm{~s}, 3 \mathrm{H}) .{ }^{13} \mathrm{C} \mathrm{NMR}\left(101 \mathrm{MHz}, \mathrm{CDCl}_{3}\right) \delta 198.9,198.4,144.0,136.8,134.3,133.2,129.3$, 128.6, 128.3, 128.2, 32.6, 32.5, 21.7.HRMS (ESI) calcd for $\mathrm{C}_{17} \mathrm{H}_{16} \mathrm{NaO}_{2},[\mathrm{M}+\mathrm{Na}]^{+} \mathrm{m} / \mathrm{z}=$ 275.1043, found: 275.1048 . Analytical data is in accordance with literature data. ${ }^{49}$

1-(4-methoxyphenyl)-4-phenylbutane-1,4-dione (3E)<smiles>COc1ccc(C(=O)CCC(=O)c2ccccc2)cc1</smiles>

Prepared following the general procedure E employing (1-bromovinyl) benzene (104 mg, $0.5 \mathrm{mmol}, 1.0$ equiv.) and 2-mercapto-1-(4-methoxyphenyl) ethan-1-one (125 mg, 0.75 mmol, 1.5 equiv.) as substrates. The desired product was obtained as light-yellow solid (121 $\mathrm{mg}$, yield 91\%) after purification by flash column chromatography on silica gel (petroleum ether/ ethyl acetate $=30 / 1-10 / 1) .{ }^{1} \mathrm{H} \operatorname{NMR}\left(400 \mathrm{MHz}, \mathrm{CDCl}_{3}\right) \delta 8.03(\mathrm{t}, J=7.6 \mathrm{~Hz}, 4 \mathrm{H})$, $7.58(\mathrm{t}, J=7.3 \mathrm{~Hz}, 1 \mathrm{H}), 7.48(\mathrm{t}, J=7.6 \mathrm{~Hz}, 2 \mathrm{H}), 6.95(\mathrm{~d}, J=8.8 \mathrm{~Hz}, 2 \mathrm{H}), 3.88(\mathrm{~s}, 3 \mathrm{H})$, 3.49-3.38 (m, 4H). ${ }^{13} \mathrm{C}$ NMR $\left(101 \mathrm{MHz}, \mathrm{CDCl}_{3}\right) \delta 198.9,197.2,163.6,136.9,133.1,130.4$, 129.9, 128.6, 128.2, 113.8, 55.5, 32.7, 32.3. HRMS (ESI) calcd for $\mathrm{C}_{17} \mathrm{H}_{17} \mathrm{O}_{3}[\mathrm{M}+\mathrm{H}]^{+} \mathrm{m} / \mathrm{z}=$ 269.1172, found: 269.1170 . Analytical data is in accordance with literature data. ${ }^{49}$ 


\section{References}

1. Lowry, M. S.; Goldsmith, J. I.; Slinker, J. D.; Rohl, R., Pascal; R. A.; Malliaras, G. G.\& Bernhard, S. Single-Layer Electroluminescent Devices and Photoinduced Hydrogen Production from an Ionic Iridium (III) Complex. Chem. Mater. 2005, 17, 5712-5719.

2. Liu, Y.; Yin, Y.; Zhang, Z.; Li, C. J.; Zhang, H.; Zhang, D.; Jiang, C.; Nomie, K.; Zhang, L.; Wang, M. L. \& Zhao, G. Structural optimization elaborates novel potent Akt inhibitors with promising anticancer activity. Eur. J. Med. Chem. 2017, 138, 543-551.

3. Matsushita, Y.; Ochi, R.; Tanaka, Y.; Koike, T.; Akita, M.; Energy transfer-driven regioselective synthesis of functionalized phenanthridines by visible-light Ir photocatalysis. Org. Chem. Front., 2020, 7, 1243-1248

4. Jing, Q; Feng-Lian, Z.; Ji-Kang, J.; Qiang, Z.; Bin, L; Lin-Xuan, L.; Yi-Feng W. New radical borylation pathways for organoboron synthesis enabled by photoredox catalysis. Angew. Chem. Int. Ed. 2020, 59, 12876 - 12884.

5. Sylvie D.; Hubert K.; Christian B. Selective Ruthenium-Catalyzed Hydrochlorination of Alkynes: One-Step Synthesis of Vinyl chlorides. Angew. Chem., Int. Ed. 2015, 54, 12112-12115.

6. Ojha, P.D.; Prabhu R.K. Regioselective synthesis of vinyl halides, vinyl sulfones, and alkynes: a tandem intermolecular nuclephilic and electrophilic vinylation of tosylhydrazones. Org. Lett. 2015, $17,18-21$.

7. Xu, C. X.; Ma, . C. H.; Xiao, F. R.; Chen H. W.; Dai B. Catalyst-free hydrochlorination protocol for terminal arylalkynes with hydrogen chloride. Chinese. Chem. Lett. 2016, 27, 1683 - 1685.

8. Pyle, L. J.; Shaffer, A. A.; Cantrell, S. J. An approach to the synthesis of unsymmetrically substituted chlorobiphenyls. J. Org. Chem, 1981, 46, 115 - 118.

9. Gerard, D. M.; Elizabeth, S. D.; David, C.; Anthony, J. J.; John, M. H.; Johnson, K. J. PCT Int. Appl. (1991), WO 9119695 A2 19911226.

10. Suta, K.; Turks, M. In (III) and Hf(IV) triflate-catalyzed hydration and catalyst-free hydrohalogenation of aryl acetylenes in liquid sulfur dioxide. ACS Omega, 2018, 3, 18065 - 18077.

11. Koch, F. H.; Lodder, G.; Koch, G. J.; Bogdan, J. D.; Brown, H. G.; Carlson, A. C.; Dean, B. A.; Hage, R.; Han, P.; Hopman, C. P. J.; James, A. L.; Knape, M. P.; Roos, C. E.; Sardina, L. M.; Sawyer, A. R ; Scott, O. B.; Testa, A. C.; Wickham, D.S. Use of kinetic isotope effects in mechanism studied. 5. Isotope effects and element effects associated with hydron-transfer steps during alkoxide - promoted dehydrohalogenations. J. Am. Chem. Soc. 1997, 119, 9965 - 9974.

12. Kauffmann, T.; Stach, D. Hobe uberlegenheit der reagenzien $\mathrm{Me}_{4} \mathrm{CoLi}_{2}$ and $\mathrm{Me}_{4} \mathrm{~F}_{2} \mathrm{Li}_{2}$ im Vergleich zu $\mathrm{Me}_{2} \mathrm{CuLi}$ and $\mathrm{Me}_{4} \mathrm{MnLi}_{2}$ bei kreuzkupplungen mit vinylbromiden. Chem. Ber, 1992, 125, 913 921.

13. Meseguer, O. J.; Carbo, D. A.; Boronat, M.; Perez, L. A.; Corma, A. Partial reduction and selective transfer of hydrogen chloride on catalytic gold nanoparticles. Angew. Chem. Int. Ed. 2017, 56, 6435 6439.

14. Pesti, A. J.; Downard, A. J.; Lauritsen, D. M.; Kauffman, S. G.; Bryant III, m. w.; Huhn, F. G.; Amett, F. J.; Yule, E. R.; Segretario J.; Nelson, A. K.; Gorko, F. E.; Page, O. G.; Lloyd, M. L. Synthesis of the methanesulfonates

of

$\alpha$-(4-Chlorophenyl) 
ethenyl]-1H,1,2,4-triazole-1-ethanol and $\alpha$-[1-2-chlorophenyl] ethenyl]$\alpha$-(2,4-difluorophenyl)-1H-1, 2, 4-triazole-1-ethanol, alpha styryl carbinol antifungal agents. J. Heterocyclic Chem, 1998, 35, 249 - 255.

15. Ghaffarzadeh M.; Bolourtchian, M.; Zohreh, M. H.; Halvagar, R.; Mohsenzadeh, F. One-Step Synthesis of Aromatic Terminal Alkynes from Their Corresponding Ketones under Microwave Irradiation. Synthetic Commu. 2006, 36(14), 1973 - 1981.

16. Chen X.; Chen T.; Xiang Y.; Zhou Y.; Han D.; Han L.; Yin S. Metal-free regioselective hydrobromination of alkynes through $\mathrm{CH} / \mathrm{CBr}$ activation. Tetrahedron Lett. 2014, 55, 4572 - 4575.

17. Castro, G. A.; Xiao, J.L. Green and efficient: iron-catalyzed selective oxidation of olefins to carbonyls with $\mathrm{O}_{2}$. J. Am. Chem. Soc. 2015, 137(25), 8206 - 8218.

18. Cai, G. L.; Zhou, Z.B.; Wu, W.C.; Yao, B.; Zhang, S.W.; Li, X.F. Pd-Catalyzed C(sp3)-C(sp2) cross-coupling of $\mathrm{Y}\left(\mathrm{CH}_{2} \mathrm{SiMe}_{3}\right)_{3}(\mathrm{THF})_{2}$ with vinyl bromides and triflates. Org. Biomol. Chem. 2016, 14, 8702 - 8706

19. Wu, J. L,; Yoshikai, N ; Cobalt-Catalyzed Alkenylzincation of Unfunctionalized Alkynes; Angew. Chem. Int. Ed. 2016, 55, 336 - 340.

20. Caina. L.D.; McLaughlinb, C.; Molloyb, J.J.; Warrena, C.C.; Andersonc, A. N.; Watson, J.B.A. A Cascade Suzuki-Miyaura/Diels-Alder Protocol: Exploring the Bifunctional Utility of Vinyl Bpin; Synlett, 2019, 30, $787-791$.

21. Zhang, Y.; Hatami, N.; Lange, S.N.; Ronge, E.; Schilling, W.; Jooss, C.; Das, S. A Metal-Free Heterogeneous Photocatalyst for the Selective Oxidative Cleavage of $\mathrm{C}=\mathrm{C}$ Bonds in Aryl Olefins via Harvesting Direct Solar Energy. Green Chem. 2020, 22, 4516 - 4522.

22. Liu, Y.; Yeung, Y.Y. Synthesis of Macrocyclic Ketones through Catalyst-Free Electrophilic Halogen-Mediated Semipinacol Rearrangement: Application to the Total Synthesis of ( \pm )-Muscone. Org. Lett. 2017, 19, $1422-1425$

23. Xu, M.H.; Dai, K.L.; Tu, Y.Q.; Zhang, X.M.; Zhang, F.M.; Wang, S.H. A catalytic allylic cation-induced intermolecular allylation-semipinacol rearrangement; Chem. Commun. 2018, 54, $7685-7688$.

24. Clement, T. Thienyl acetylenes; Compt. rend. 1951, 232, 2236 - 2237.

25. Giuseppe B.; Roberto C.; Giustino D.A.; Riccardo G.; Silvia L.; Mauro M.; Enrico M. A convergent approach to (R)-Tiagabine by a regio- and stereocontrolled hydroiodination of alkynes. J. Org. Biomol. 2011, 8, 3509 - 3517.

26. Taka'cs, A.; Farkas, R.; Petz, A.; Kolla'r, L.; Synthesis of 2-naphthylacrylamides and 2-naphthylacrylates via homogeneous catalytic carbonylation of 1-iodo-1-naphthylethene derivatives. Tetrahedron. 2009, 65, 4795 - 4800.

27. Chen, W.Q.; Walker, C.L.J.; Oestreich, M. Metal-Free Transfer Hydroiodination of C-C Multiple Bonds; J. Am. Chem. Soc. 2019, 141, 1135 - 1140.

28. Kawaguchi, S.I;, Ogawa, A.; Highly Selective Hydroiodation of alkynes using an iodine hydrophosphine binary system. Org. Lett. 2012, 12(9), 1893 - 1895.

29. Li, J.Y.' Laishram, D.R.; Chen, J.C.; Xu, D.D; Shi, G.R.; Lv, H.P.; Fan, R.F.; Fan, B.M. Regioselective hydroiodination of alkynes by in situ-generated HI. Asian J. Org. Chem. 2020, 9, 73 - 76

30. Tetsuro, I.; Akihiro, S.; Shigenori, M. Preparation of 1-iodovinylene derivatives. PCT Int. Cl. (2014), JP2014-31319A20140220.

31. Xiao, J.; Han, L.B. Ready access to organoiodides: Practical hydroiodination and double iodination of carbon-carbon unsaturated bonds with $\mathrm{I}_{2}$. Tetrahedron. 2019, 75, 3510 - 3515 . 
32. Kiely, S.J.; Nelson, L.L.; Boudjouk, P. A Convenient Synthesis of 1 -Bromo-8-iodonaphthalene and 1,8-Dibromonaphthalene from 8-Bromo-1 -naphthoic Acid. J. Org. Chem.,1977, 42(8), 1480.

33. Dardonville, C.; Rinaldi, E.; Barrett, M. P.; Brun, R.; Gilbert, I. H.; Hanau, S. Selective inhibition of Trypanosoma brucei 6-phosphogluconate dehydrogenase by high-energy intermediate and transition-state analogues. J. Med. Chem. 2004, 47(13), 3427 - 3437.

34. Karsisiotis, A. I.; Damblon, C.; Lassaux, P.; Papamicael, C.; Roberts, G. C. K.; Galleni, M.; Dideberg, O.; Liénard, B. t. M. R.; Garau, G.; Horsfall, L. Structural basis for the broad-spectrum inhibition of metallo- $\beta$-lactamases by thiols. Org. Bioml. Chem. 2008, 6(13), 2282 - 2294

35. Skrzyn'ska, A.; Frankowski, S.; Moczulski, M.; Drelich, P. Albrecht, L. Site-Selective and Enantioselective $\alpha, \beta, \gamma$-Functionalization of 5囚Alkylidenefuran-2 $(5 H)$ 冈ones: A Route to Polycyclic $\gamma \bowtie$ Lactones. Org. Lett. 2019, 21, 1248 - 1252.

36. Dehmel, F.; Weinbrenner, S.; Julius, H.; Ciossek, T.; Maier, T.; Stengel, T.; Fettis, K.; Burkhardt, C.; Wieland, H.; Beckers, T. Trithiocarbonates as a Novel Class of HDAC Inhibitors: SAR Studies, Isoenzyme Selectivity and Pharmacological Profiles. J. Med. Chem. 2008, 51, 3985 - 4001.

37. Jiang J.; Liu J.; Yang J.; Shao Y.; Cheng J.; Bao X.; Wan X. Cu-based carbene involved in a radical process: a new crossover reaction to construct gamma-peroxy esters and 1,4-dicarbonyl compounds. Chem. Com. (Camb) 2015, 51, 14728 - 14731.

38. Andrea V.; Valentina G.; Barbara F.; Andrea G.M.; Valeria C.; Pierluca G. $\pi$-Interactions as a tool for an easy deposition of meso-tetraferrocenylporphyrin on surfaces. New J. Chem. 2013, 37, 3535 3542.

39. Zhao J.; Li P.; Xu Y.; Shi Y.; Li F. Nickel-catalyzed transformation of diazoacetates to alkyl radicals using alcohol as a hydrogen source. Org. Lett. 2019, 21, 9386- 9390.

40. Kameoka H.; Tanaka S.; Hirao N.; Synthesis of $\gamma$-arylbutyrolactones II, Kinki Daigaku Rikogakubu Kenkyu Hokoku 1974, 9, 23-27.

41. Jiang Z.; Li Z.; Yu J.; Su W.; Liquid-assisted grinding accelerating: Suzuki-Miyaura reaction of aryl chlorides under high-speed ball-milling conditions. J. Org. Chem. 2016, 81, 10049- 10055

42. Chen L.; Chen J.; Cheng H.; Lu L.; Xian W.; Enantioselective synthesis of tetrahydrofuran derivatives by sequential Henry reaction and iodocyclization of $\gamma, \delta$ - unsaturated alcohols. Eur. J. Org. Chem. 2014, 22, 4714-4719

43. Swinnen D.; Morandi F.; Alpha-amino boronic acid derivatives, selective immunoproteasome inhibitors. PCT Int. Appl. 2013, WO2013092979A1 20130627.

44. Teng J.; Qiao Y.; Zhang Q.; Li C.; Huang M.; Tris(4-methoxylphenyl)phosphine-Catalyzed C-C Bond Formation Reaction: Mutual Addition of Aromatic Aldehydes and Ethyl Acrylate. Syn. commu. 2013, 43, 848 - 858 .

45. Fujio M.; Tanaka M.; Wu X.; Funakashi K.; Sakai K.; Suemune H. ortho-Halogeno substituents effects in asymmetric cyclization of 4-aryl-4-pentenals using a Rhodium catalyst. Chem. Lett. 1998, 27, 881 - 882 .

46. Masakazu T.; Wu X.; Masakazi F.; Ortho-halogeno substituents effects in asymmetric cyclization of 4-aryl-4-pentenals using a rhodium catalyst. Chem. Lett., 1998, 27, 881 - 882.

47. Hongu M.; Hosaka T.; Kashiwagi T.; Kono, R.; Kobayashi H. Preparation of substituted imidazoles/ oxazoles/ thiazoles as large conductance calcium - activated K channel openers. PCT Int. Appl. 2002, WO 2002083111 A2 20021024.

48. Zhao J.; Li P.; Xu Y.; Shi Y.; Li F. Nickel-catalyzed transformation of diazoacetates to allcyl radicals using alcohol as a hydrogen source. Org. Lett. 2019, 21, 9386 - 9390. 
49. Ramanathan P.; Somraj G.; Govindasamy S. Proton-coupled electron transfer: transition-metal-free selective reduction of chalcones and alkynes using xanthate/formic acid. Org. Lett., 2019, 21,2650 2653. 
5. NMR Spectra Data

2a $(\mathrm{X}=\mathrm{Cl})$ :

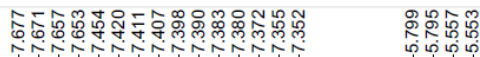

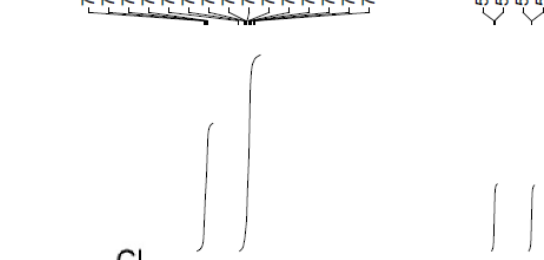

(1)

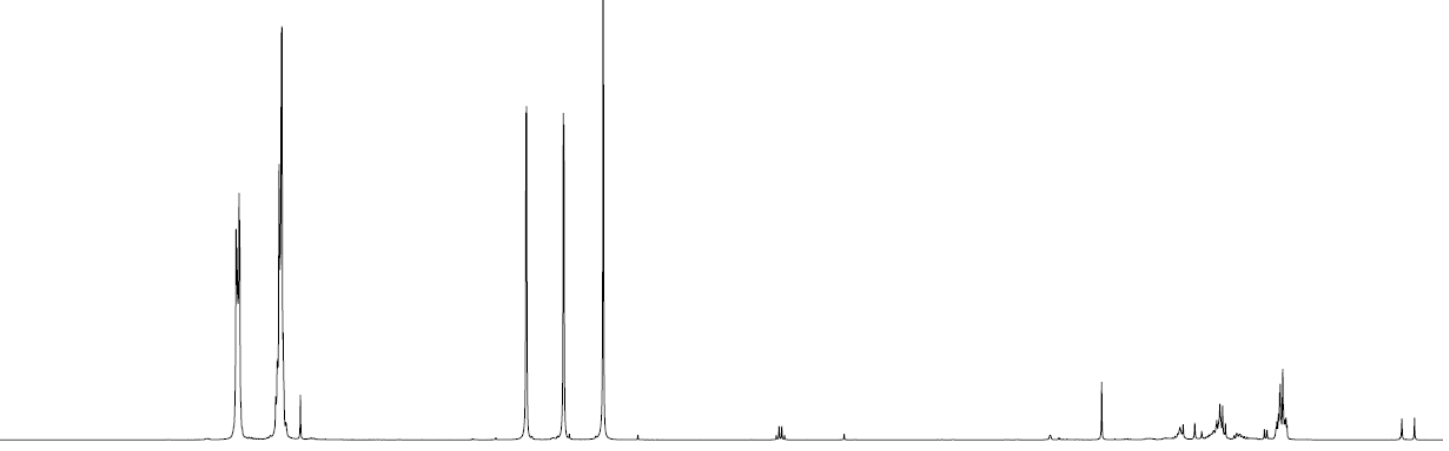

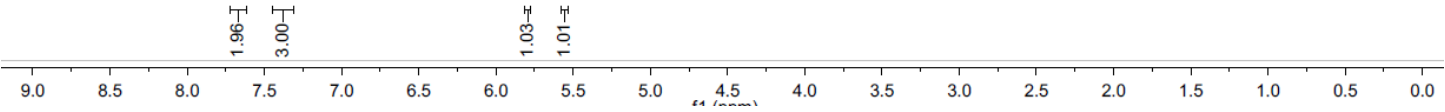


$2 b(X=C l)$ :

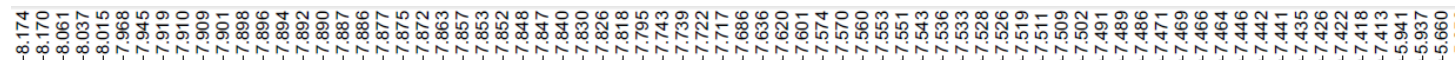
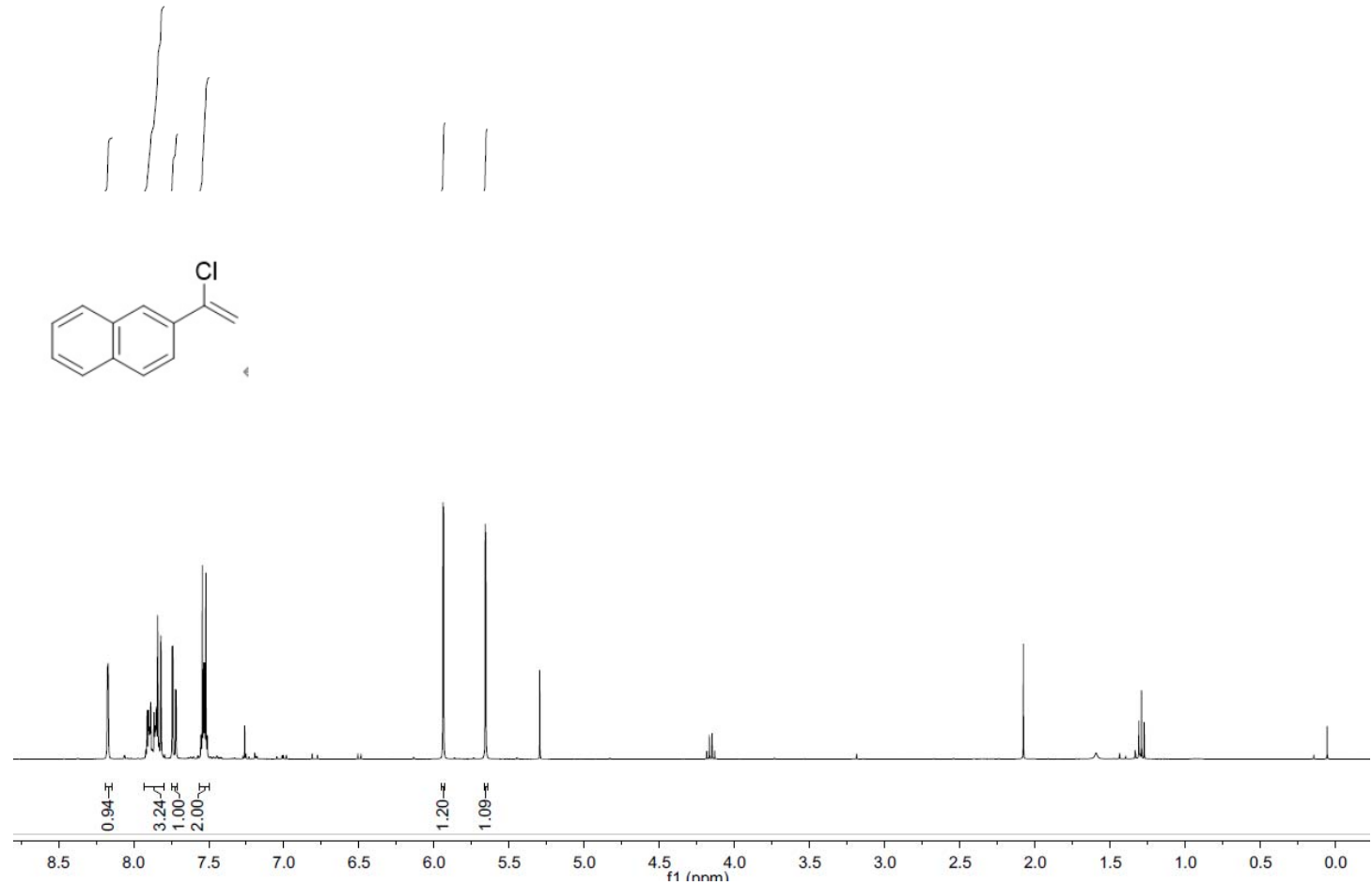

2c $(\mathrm{X}=\mathrm{Cl})$ :

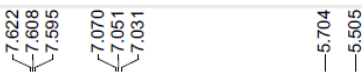

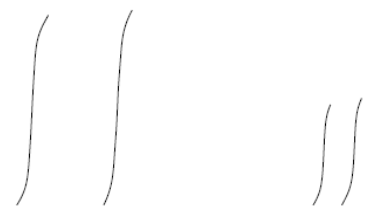

(1)

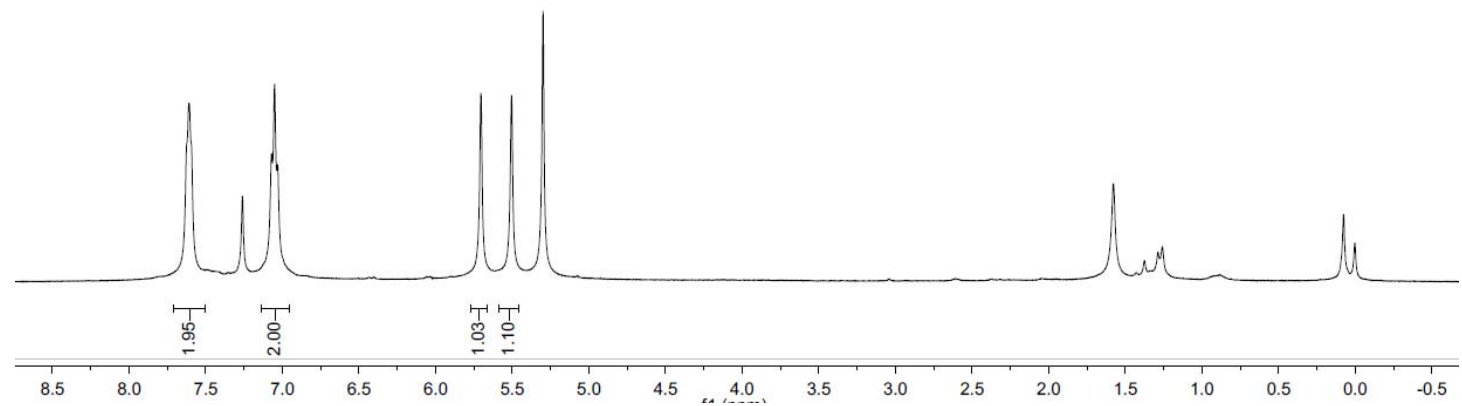


2d $(\mathrm{X}=\mathrm{Cl})$ :

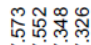

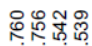

एं एं
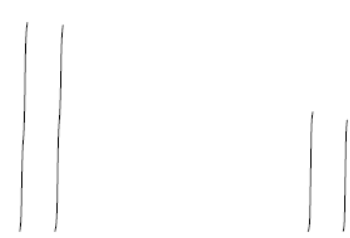

(1)

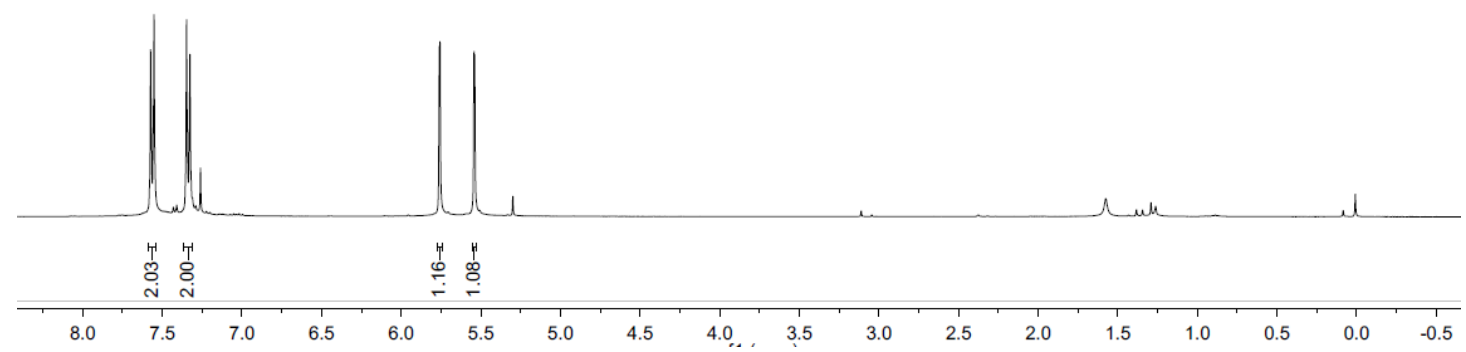

$2 \mathrm{e}(\mathrm{X}=\mathrm{Cl})$ :

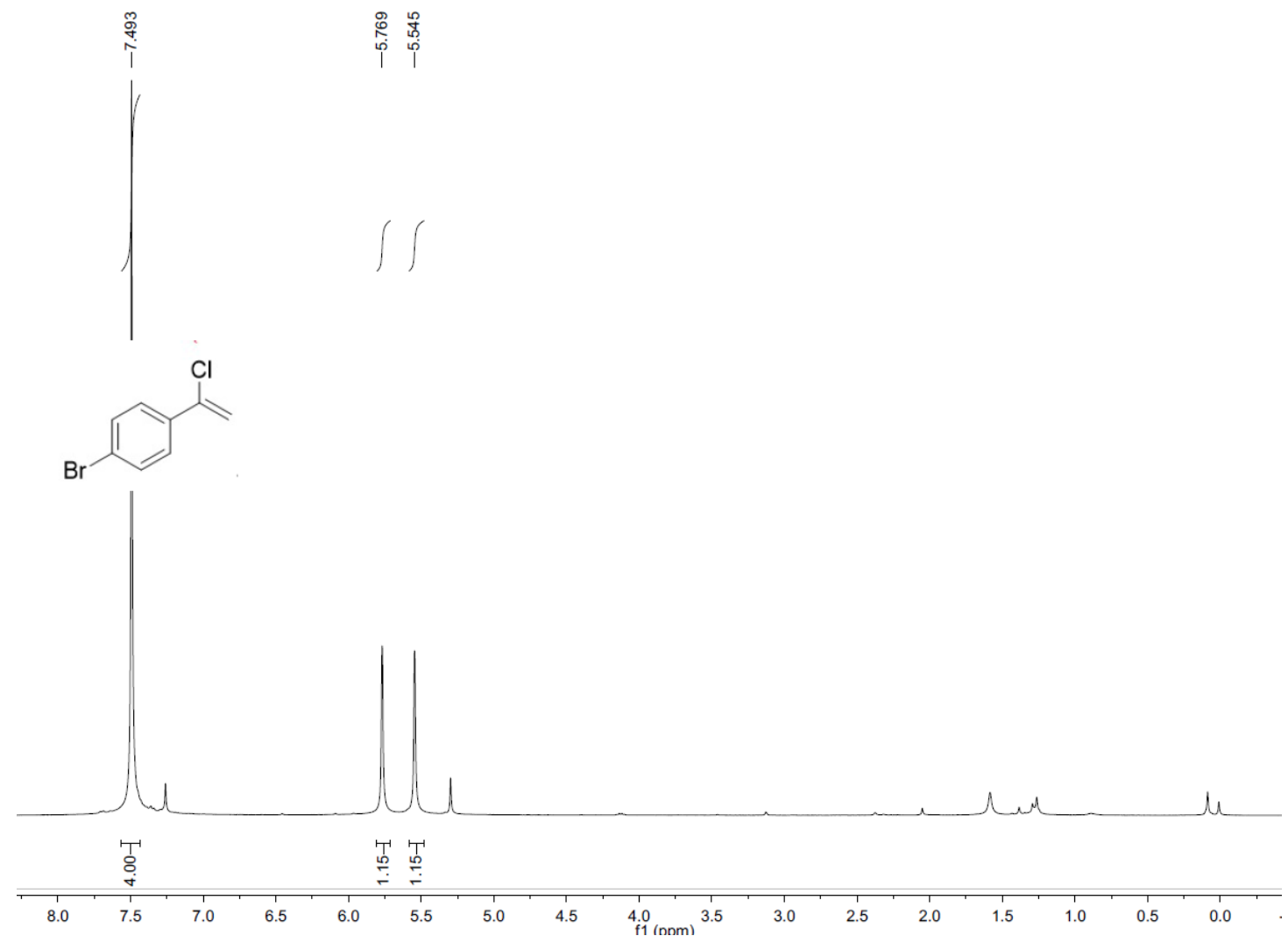


2f $(X=C l)$ :

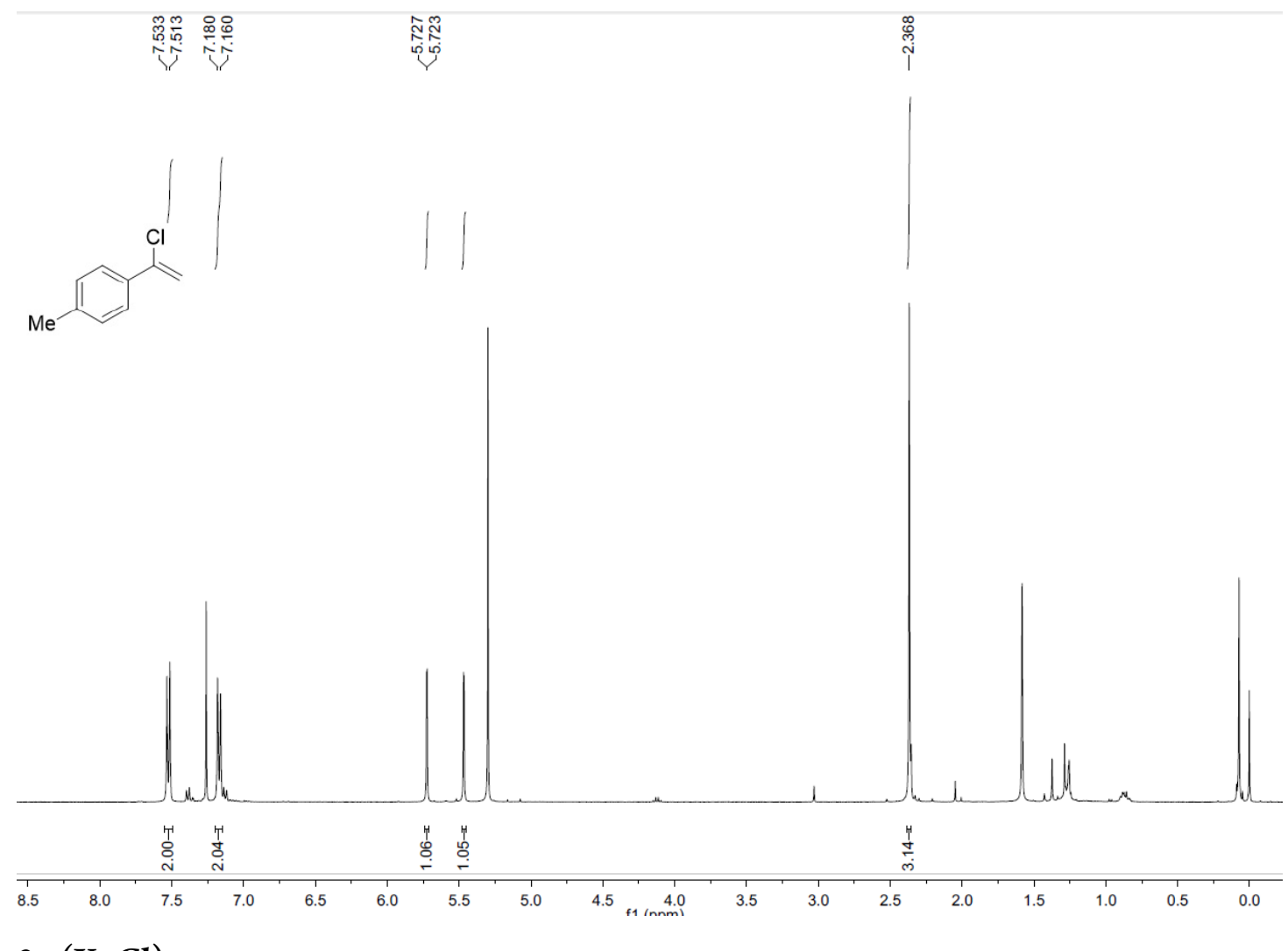

$2 \mathrm{~g}(\mathrm{X}=\mathrm{Cl})$ :

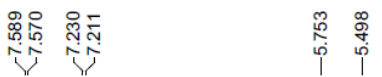

서유ำ
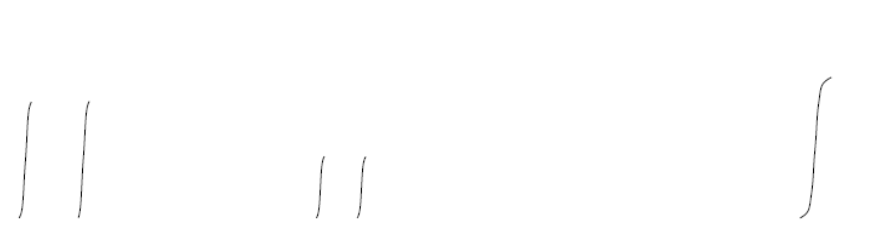

(l)
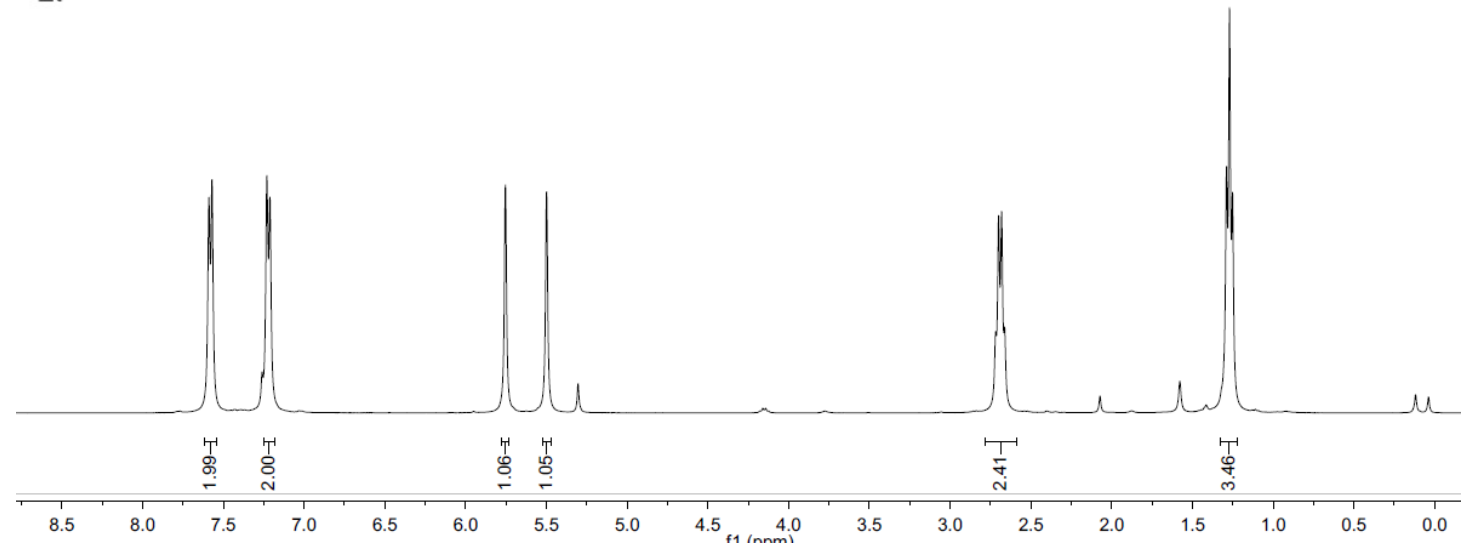
2h $(\mathrm{X}=\mathrm{Cl})$ :

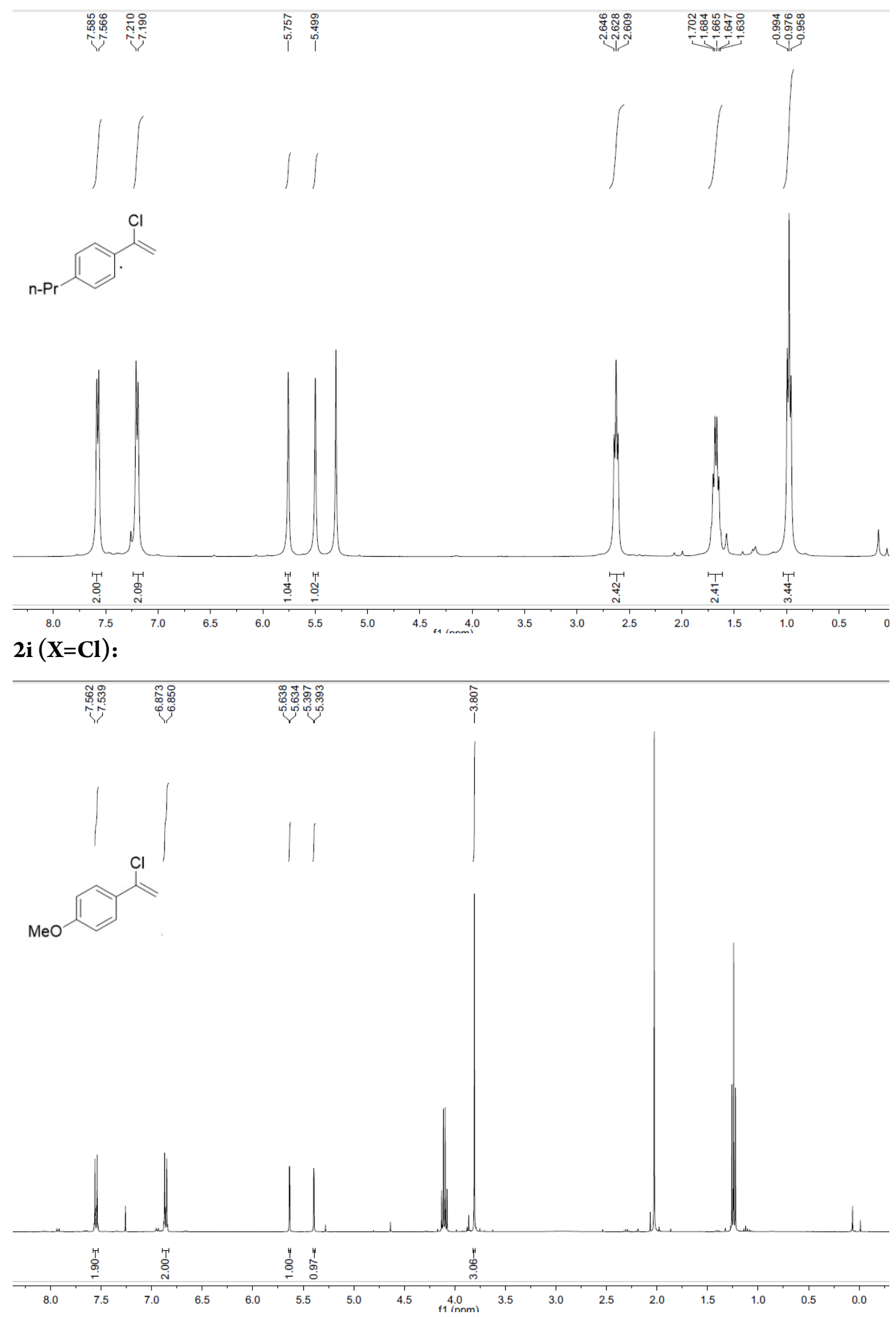


2j $(\mathrm{X}=\mathrm{Cl})$ :

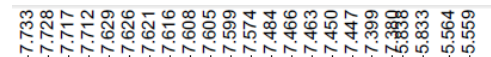

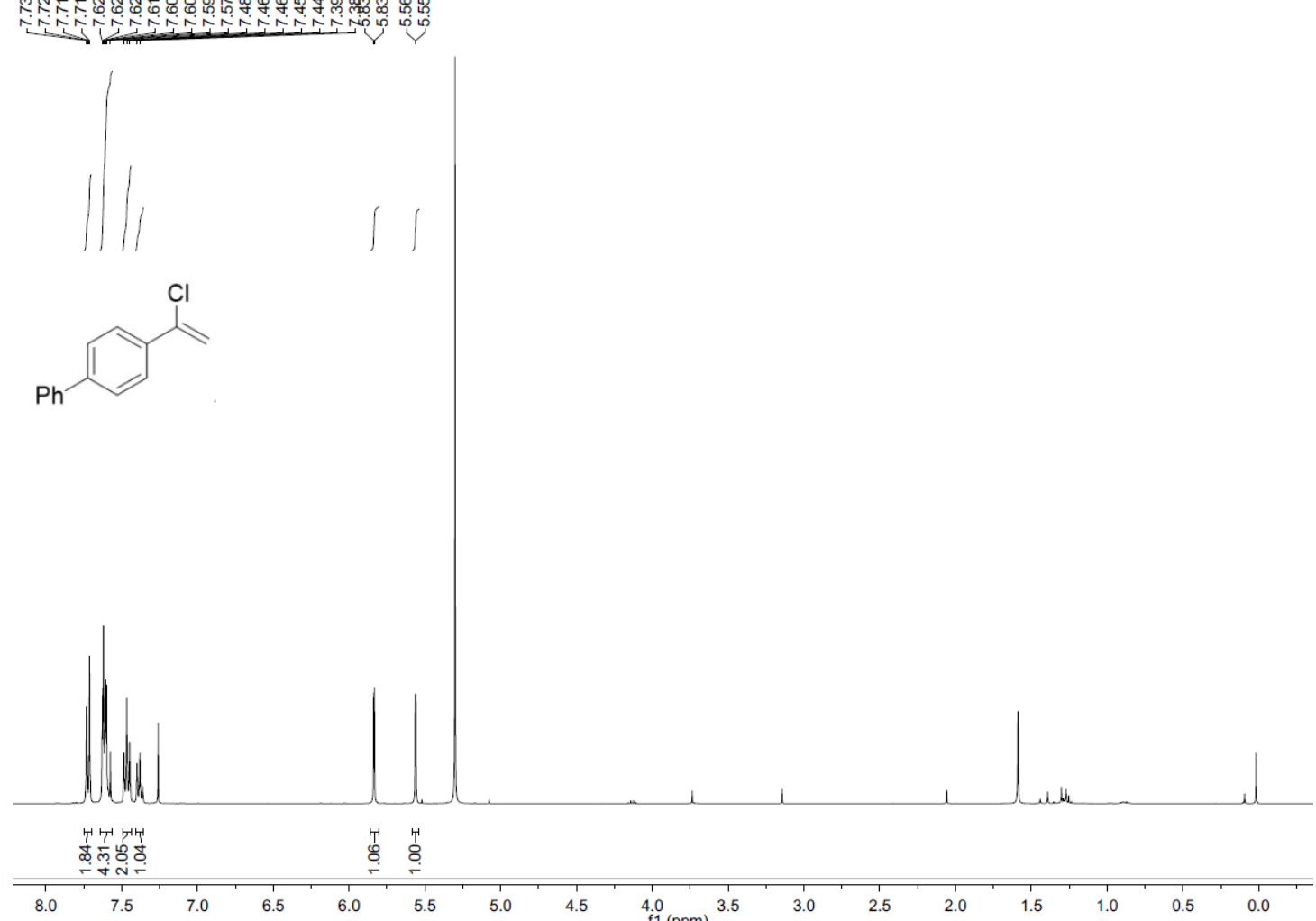

$2 \mathrm{k}(\mathrm{X}=\mathrm{Cl})$ :

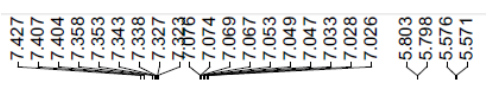
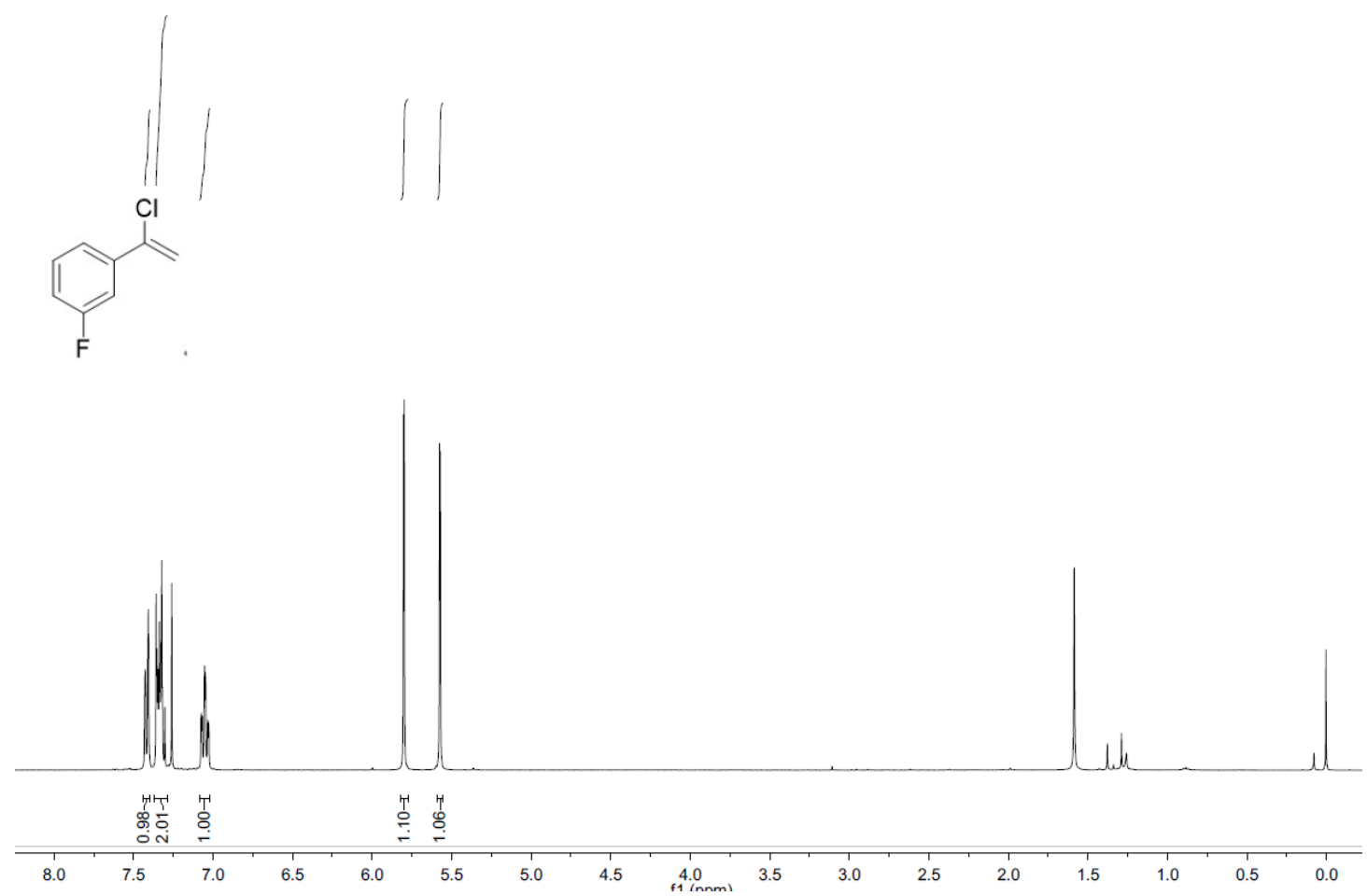
$21(\mathrm{X}=\mathrm{Cl})$ :

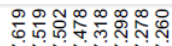

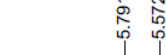
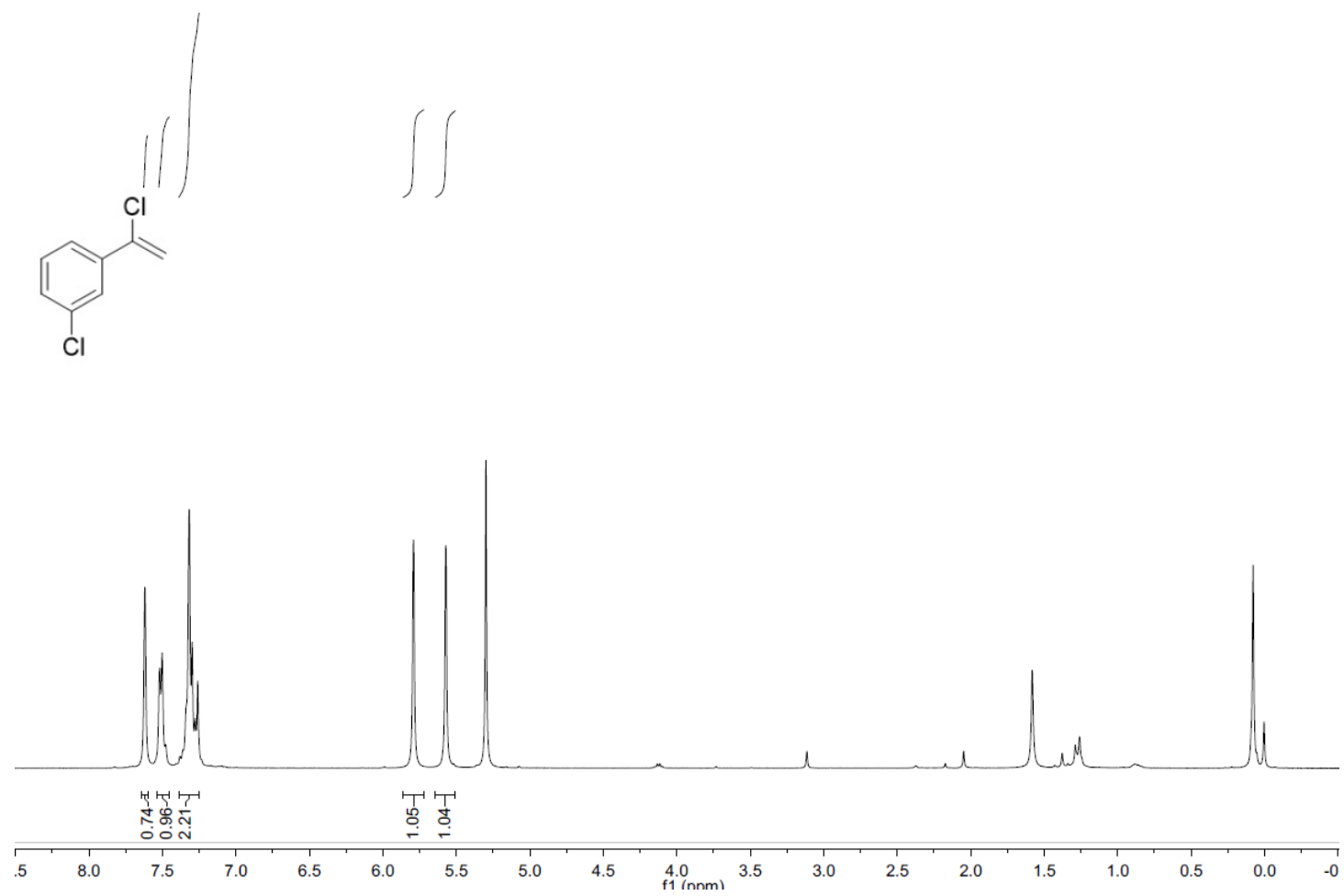

$\mathbf{2 m}(\mathrm{X}=\mathrm{Cl})$ :

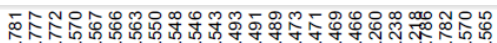

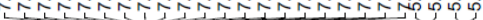
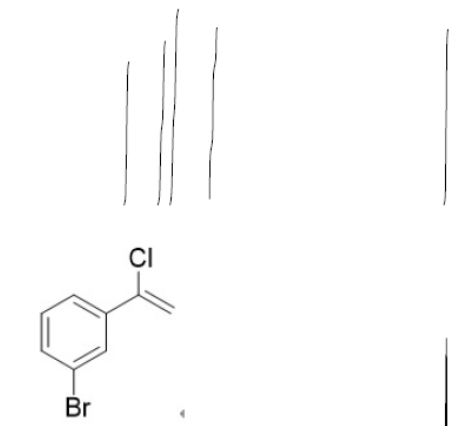
2n $(\mathrm{X}=\mathrm{Cl})$ :

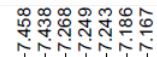

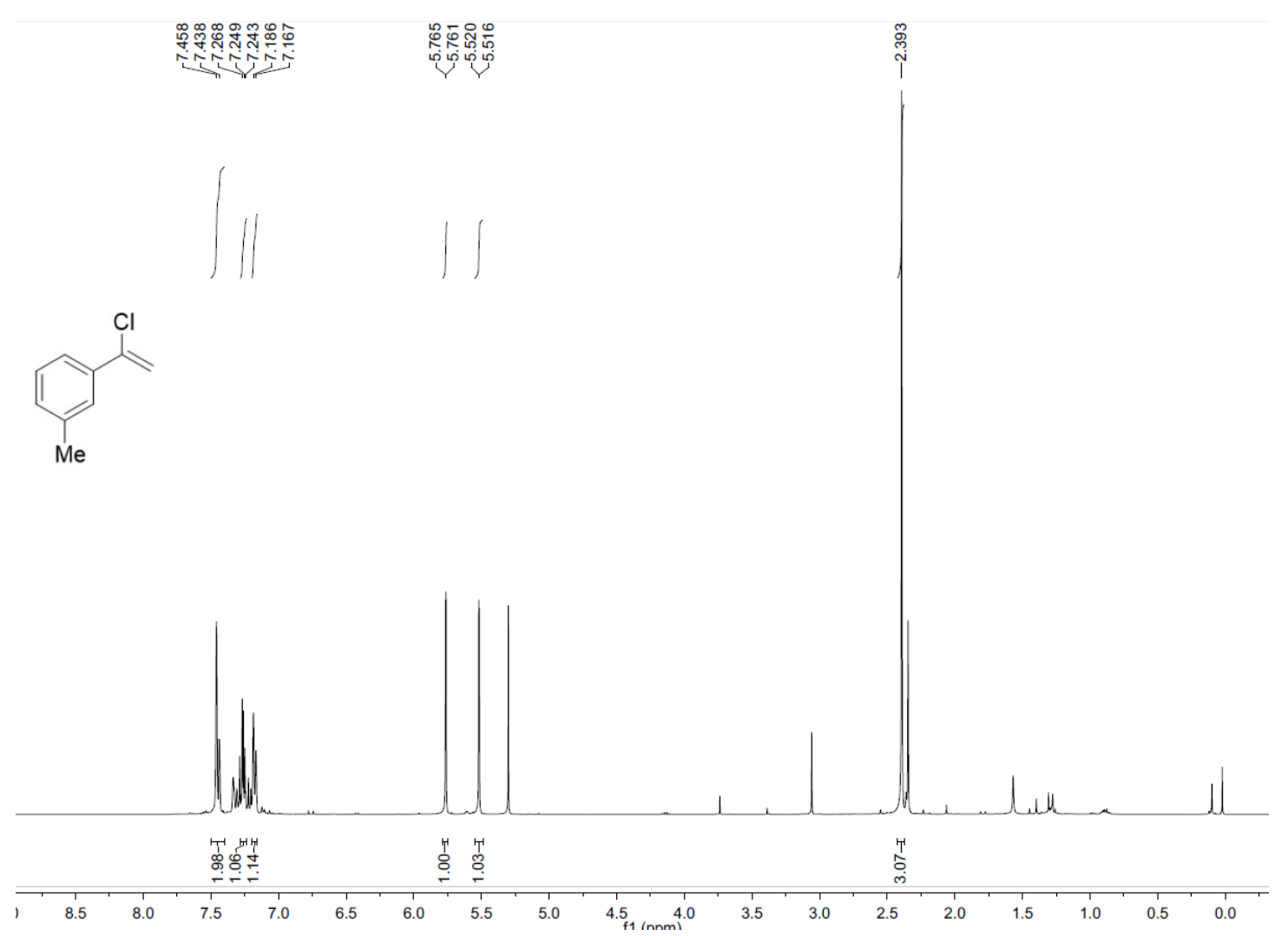

Me

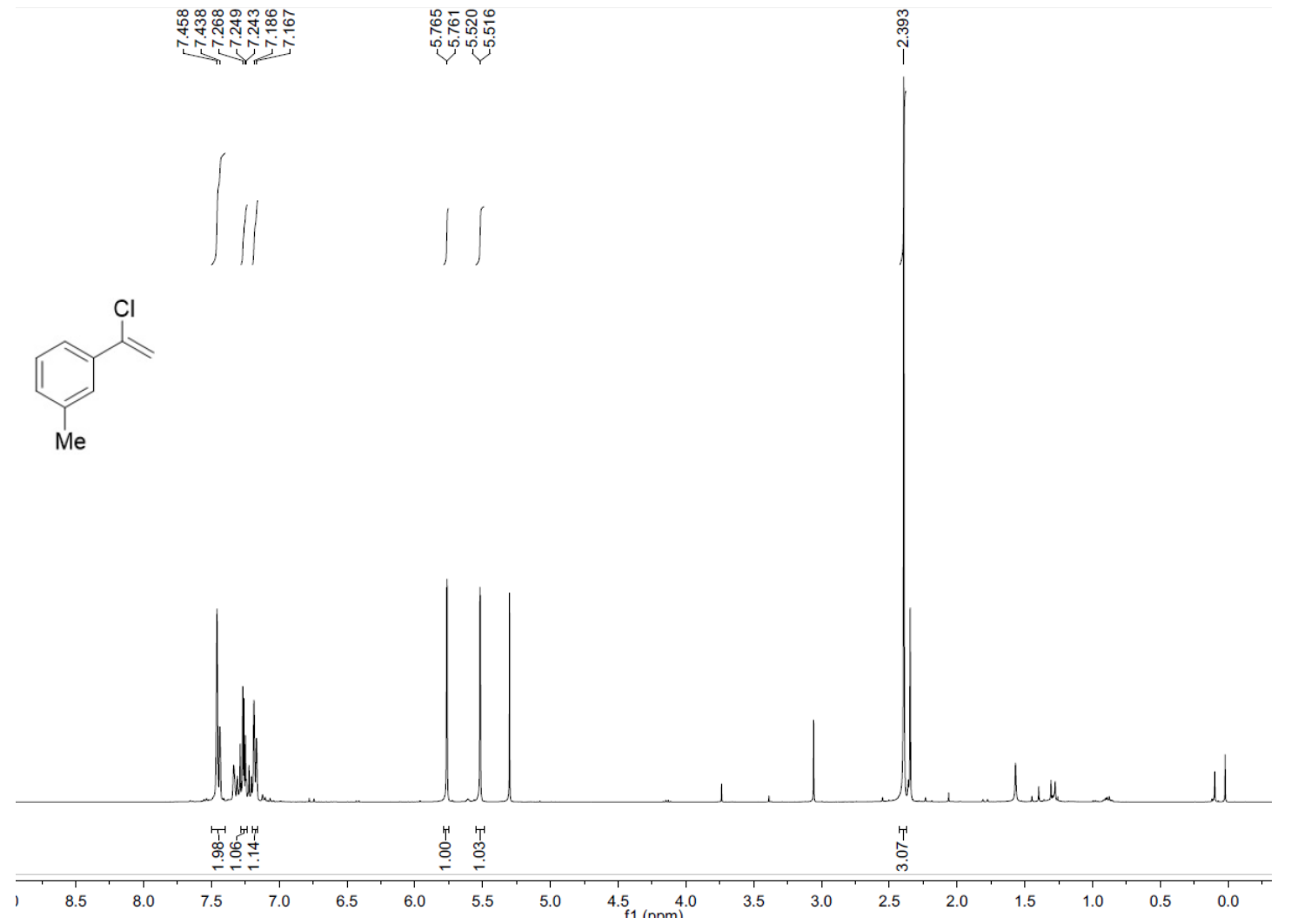

刕

$20(\mathrm{X}=\mathrm{Cl})$ :

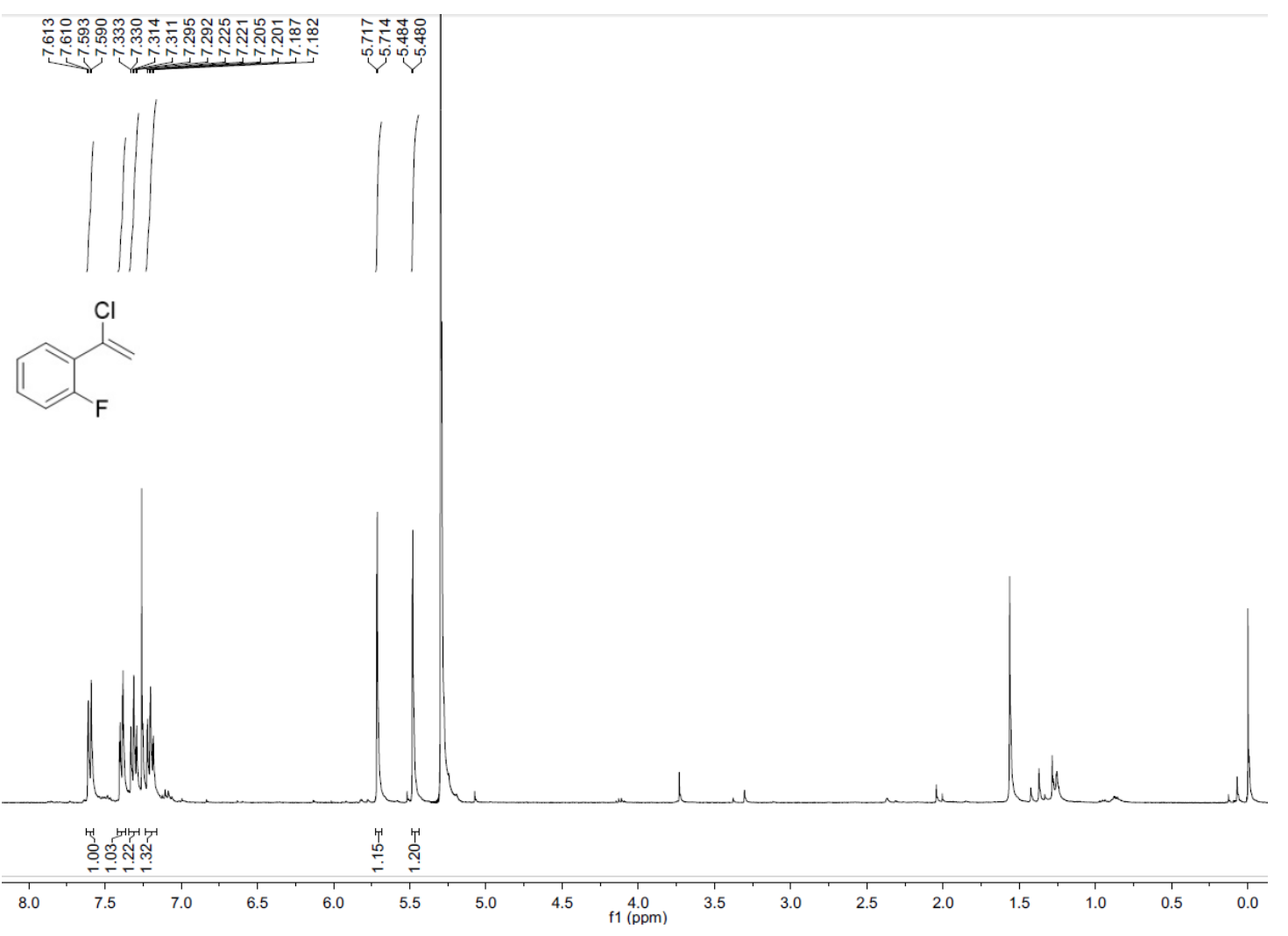




\section{2p $(\mathrm{X}=\mathrm{Cl})$ :}

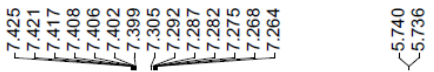
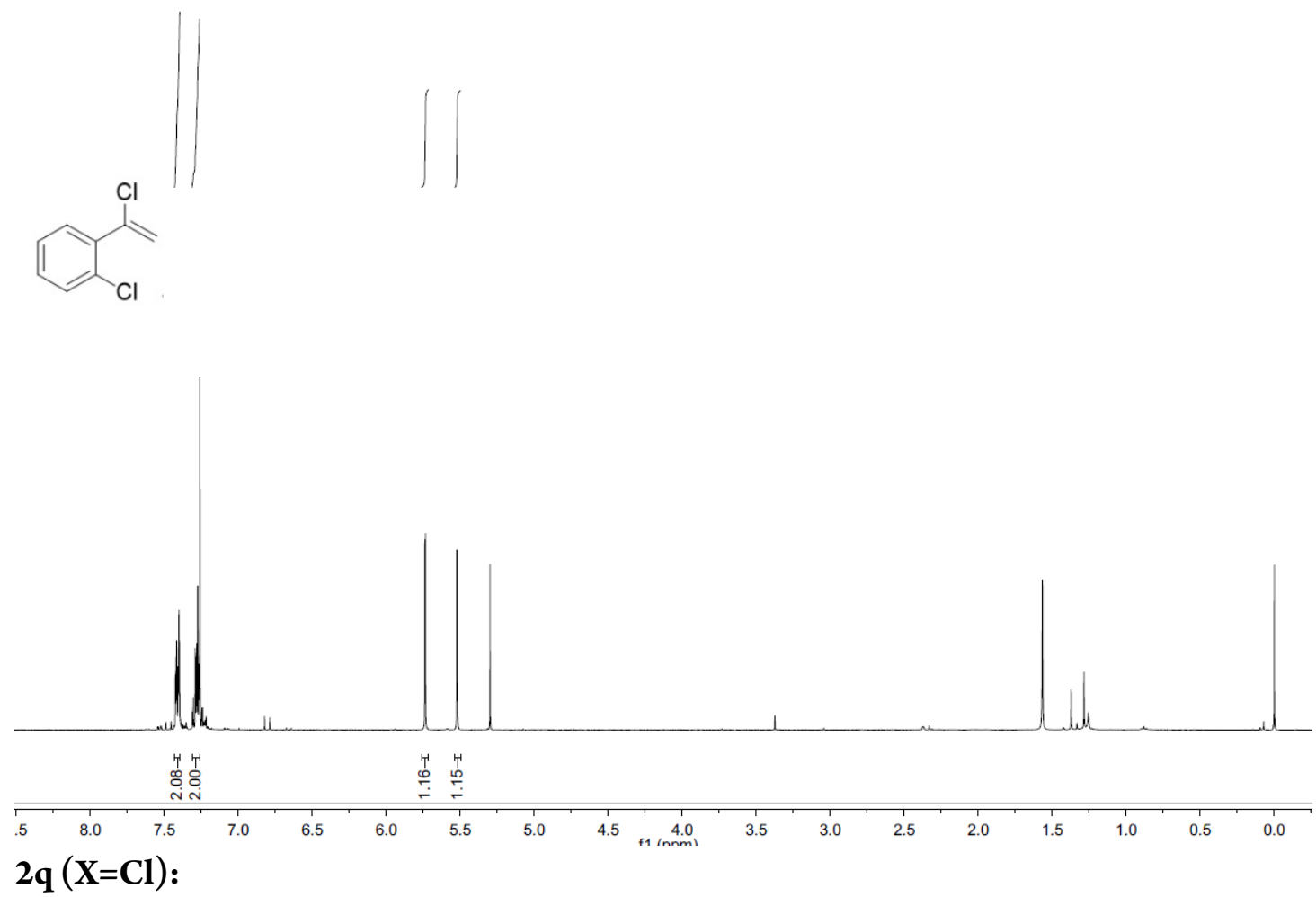

일

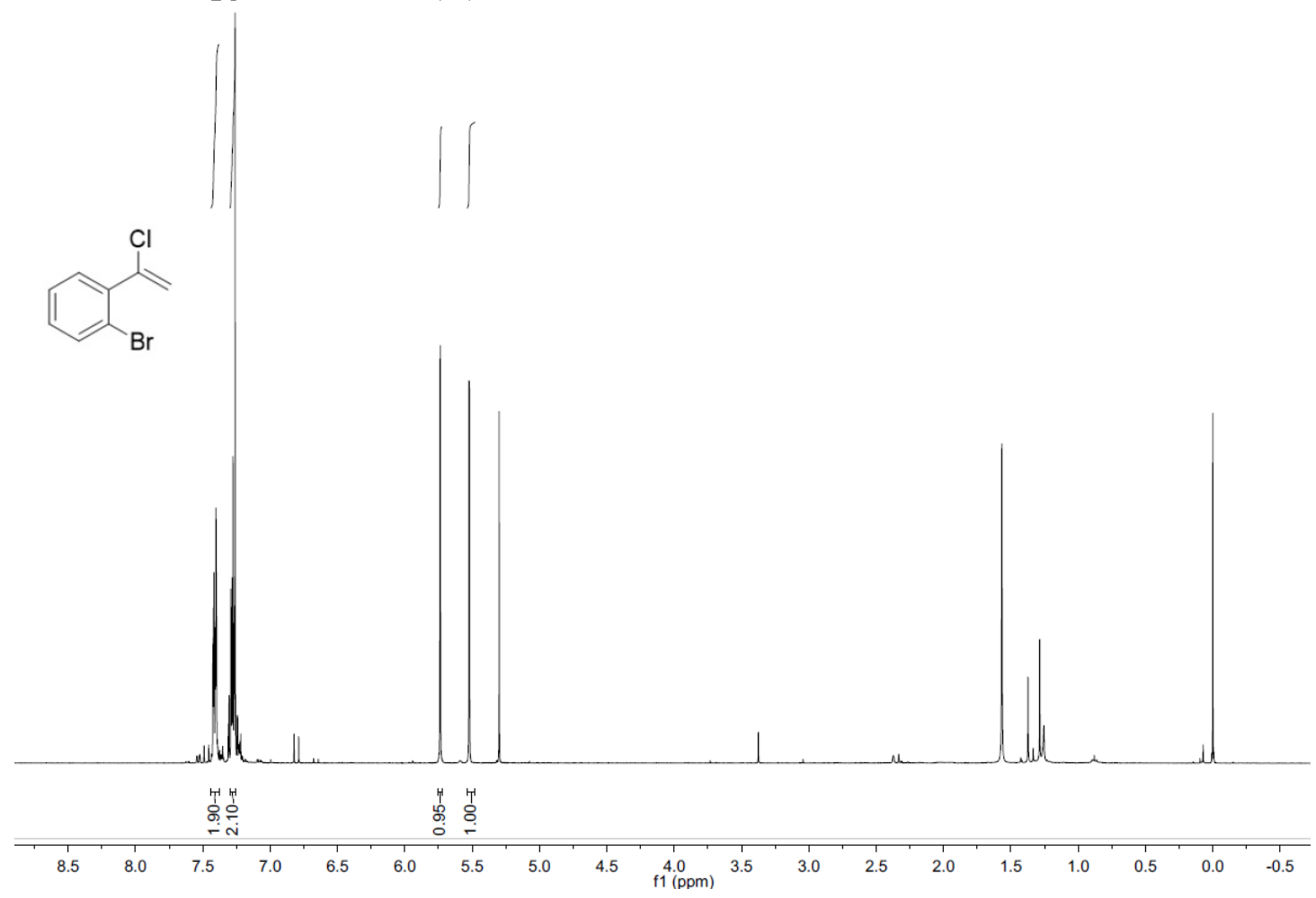


2r $(\mathrm{X}=\mathrm{Cl})$ :

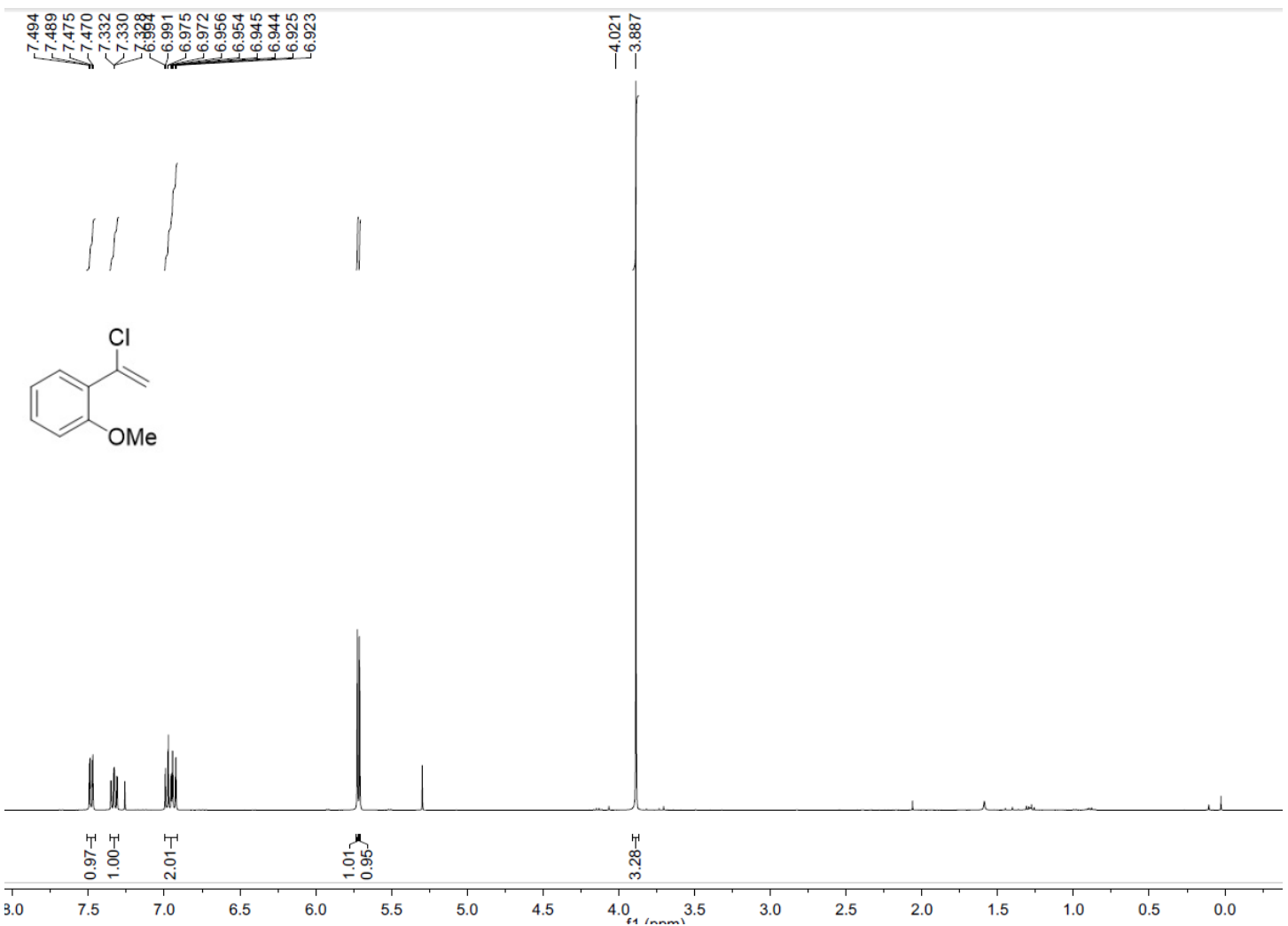

2s $(\mathrm{X}=\mathrm{Cl})$ :

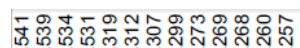

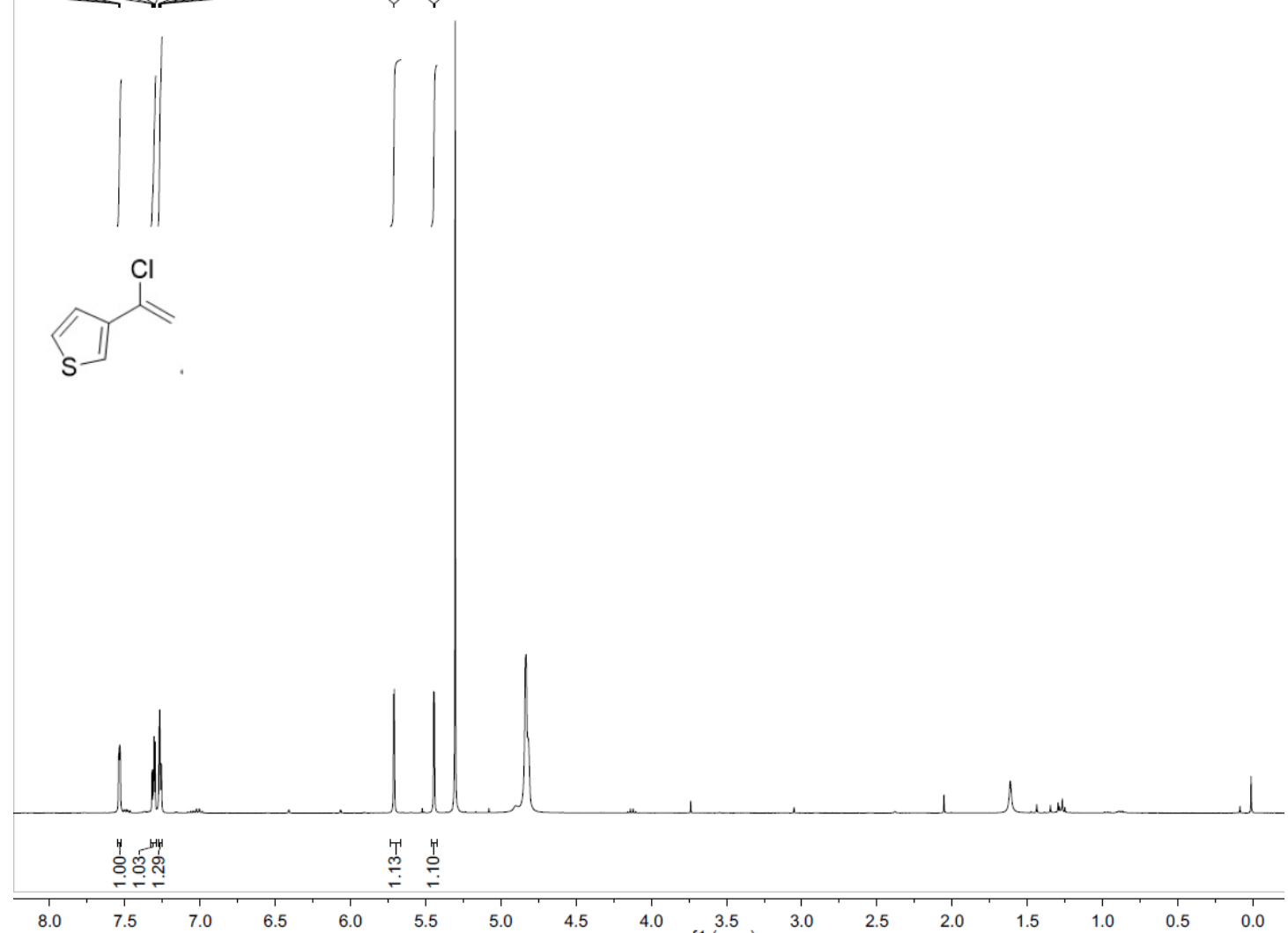


2b $(\mathrm{X}=\mathrm{Br})$ :

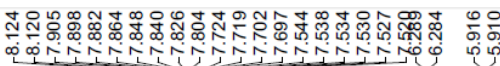

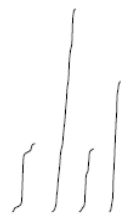

$c_{1}^{B r}$

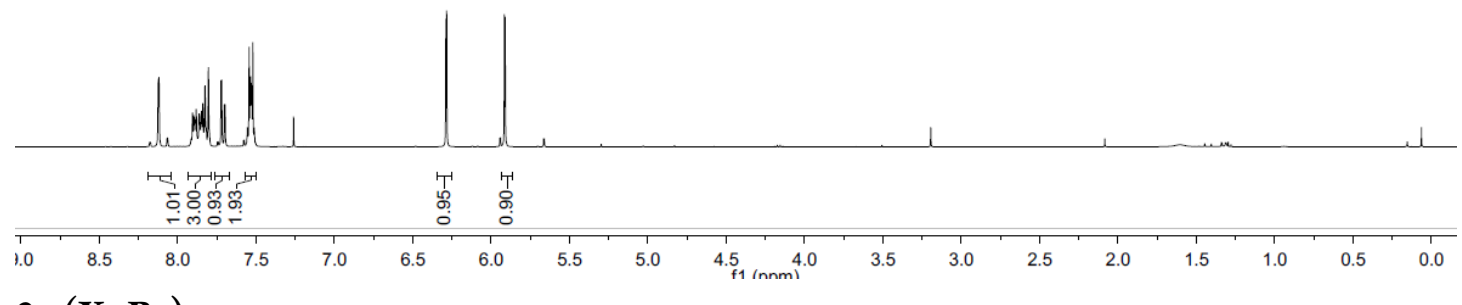

2c $(\mathrm{X}=\mathrm{Br})$ :

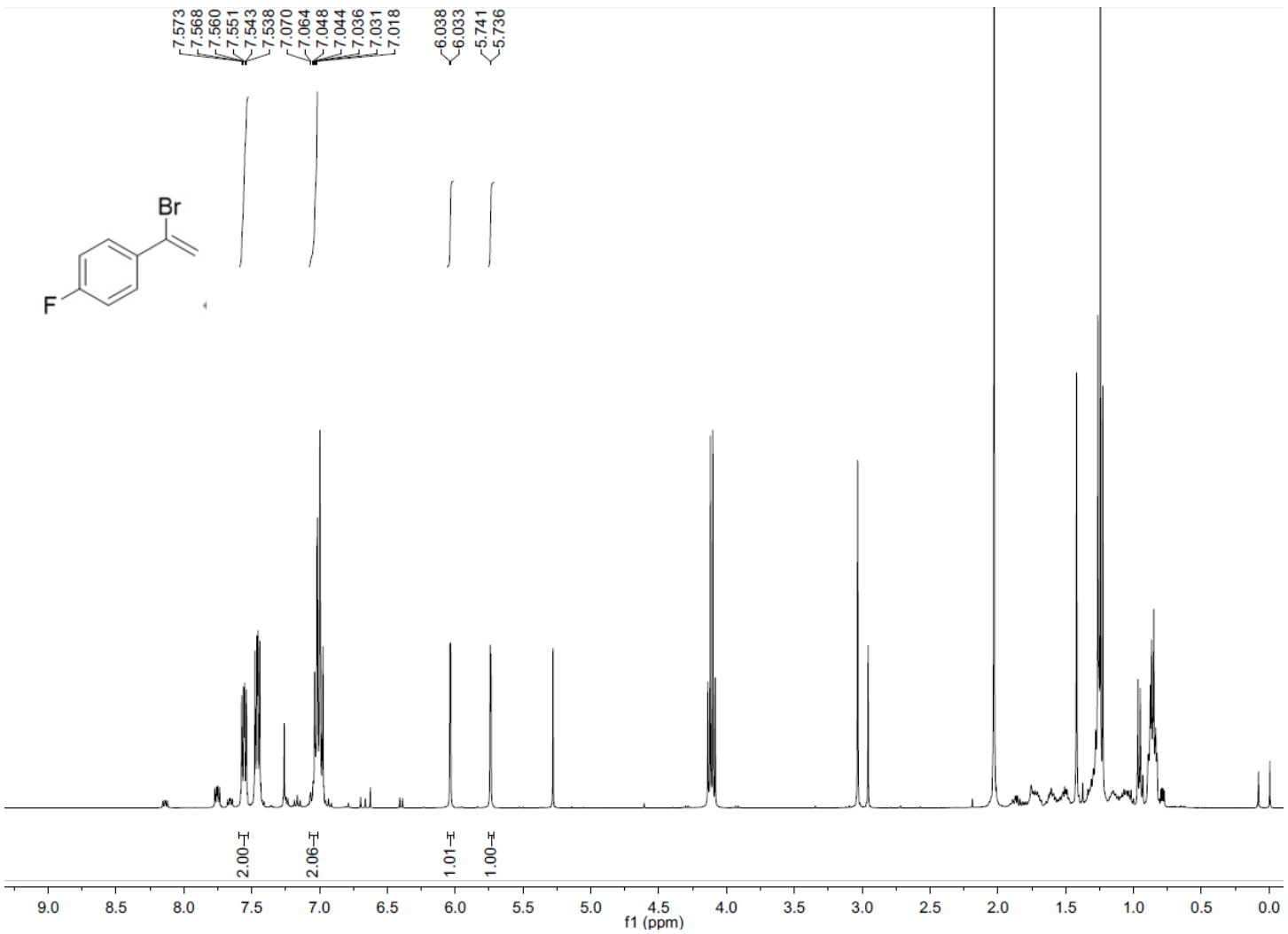


2d $(\mathrm{X}=\mathrm{Br})$ :
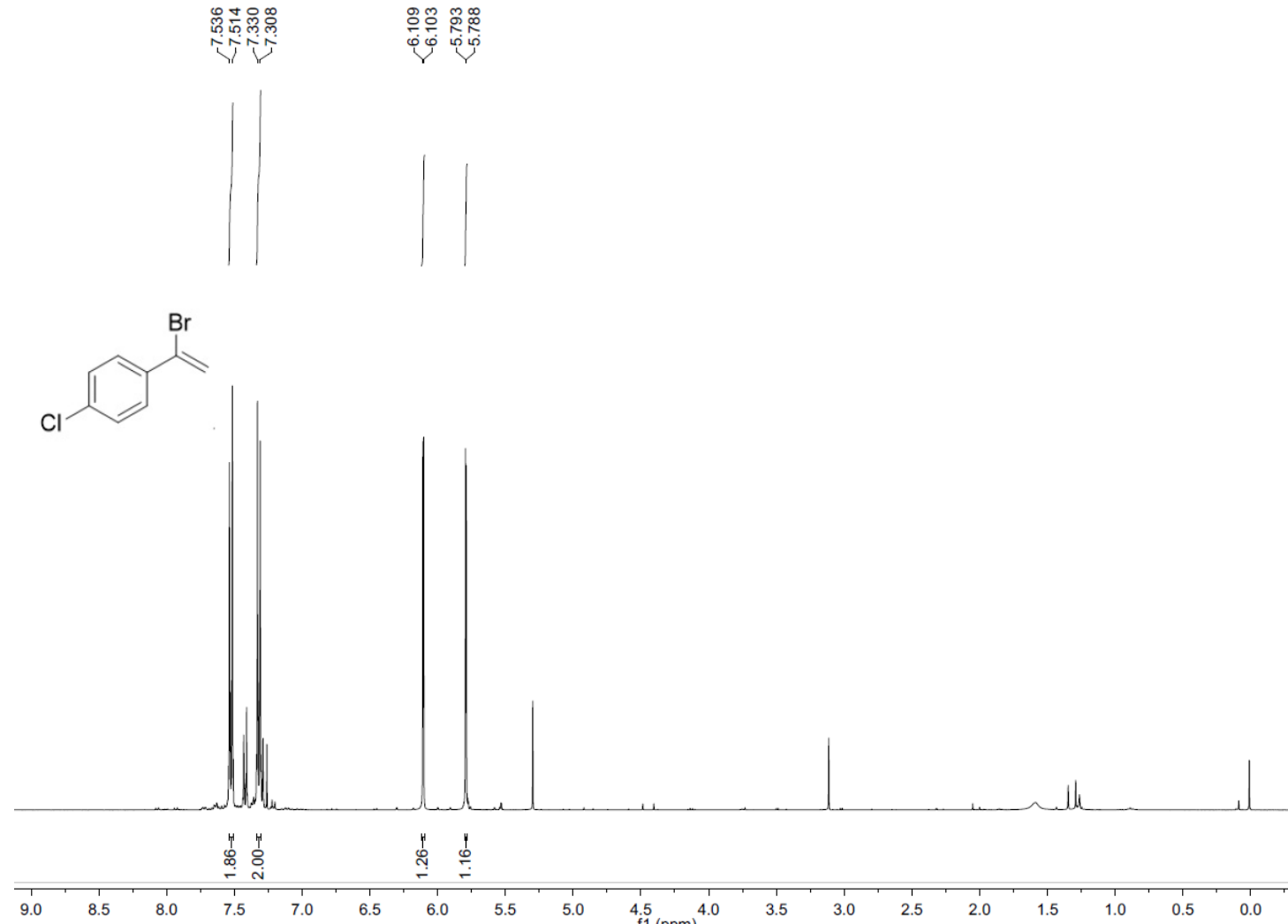

$2 e(X=B r):$

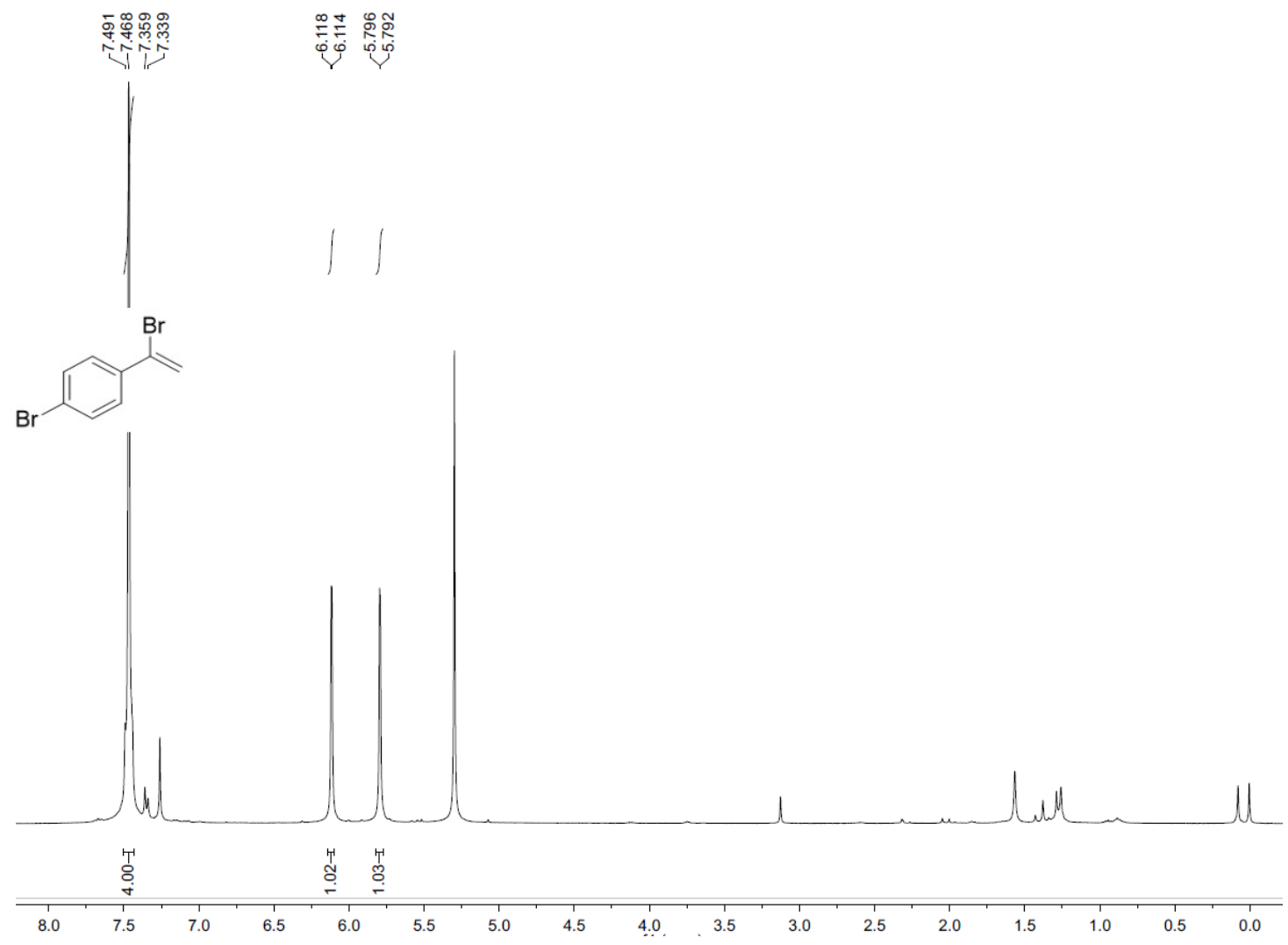


$2 f(X=B r)$ :

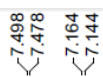

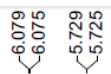
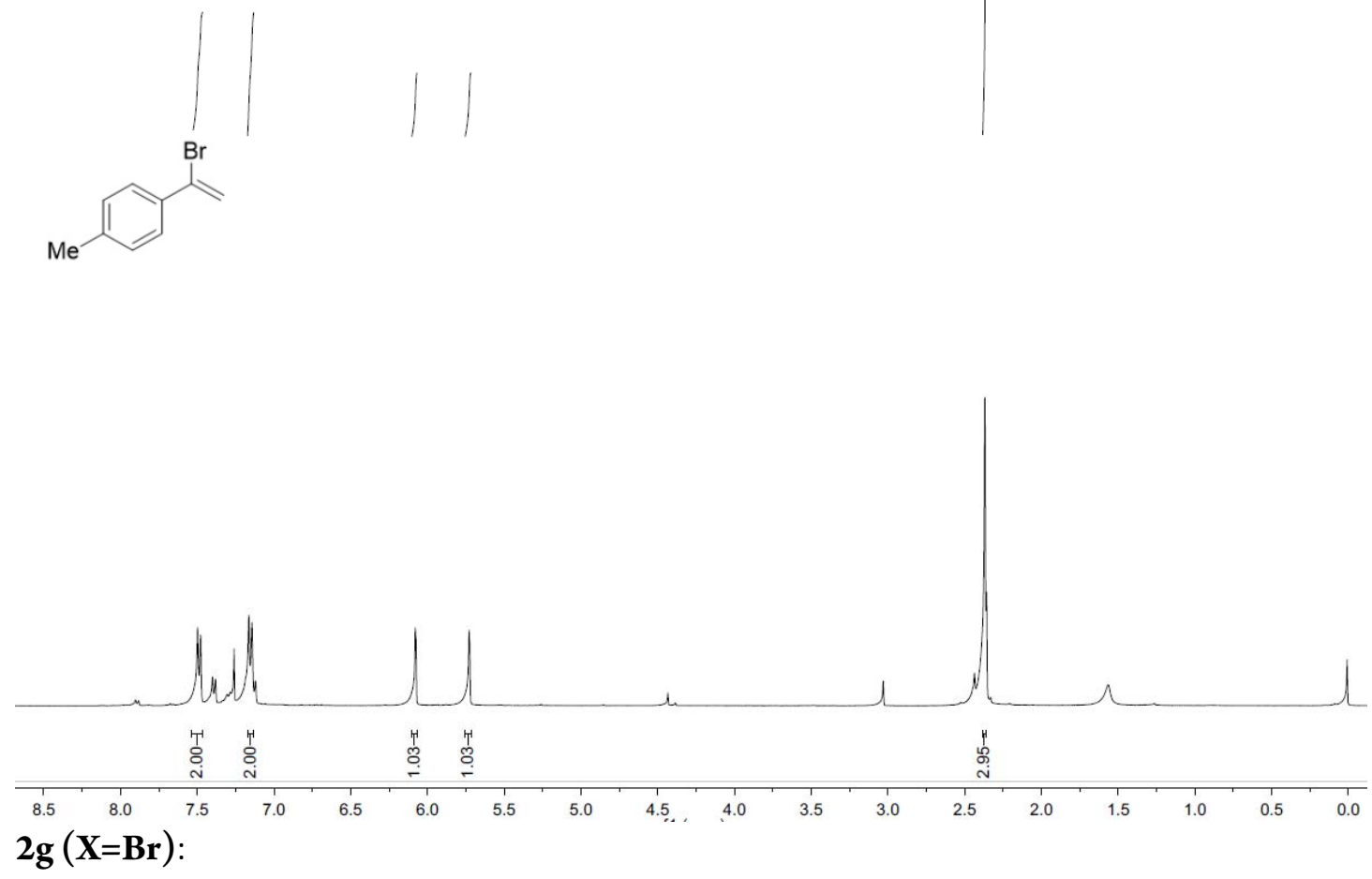

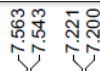

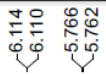

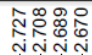

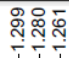

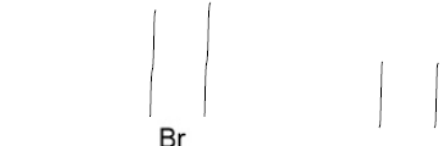<smiles>C=C(Br)c1ccc(CC)cc1</smiles>

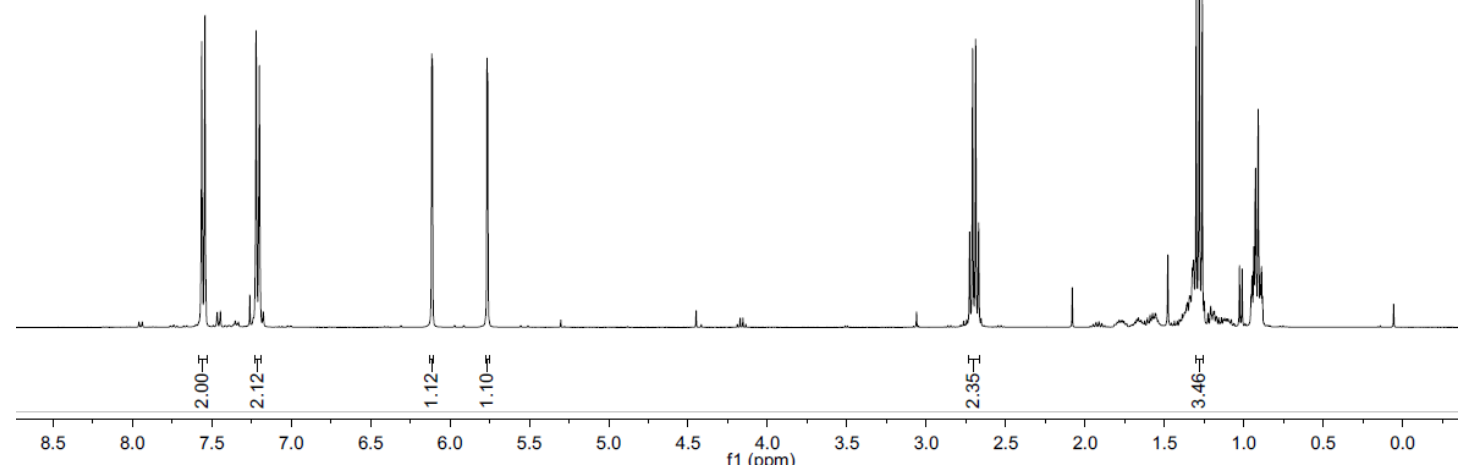


2h $(\mathrm{X}=\mathrm{Br})$ :

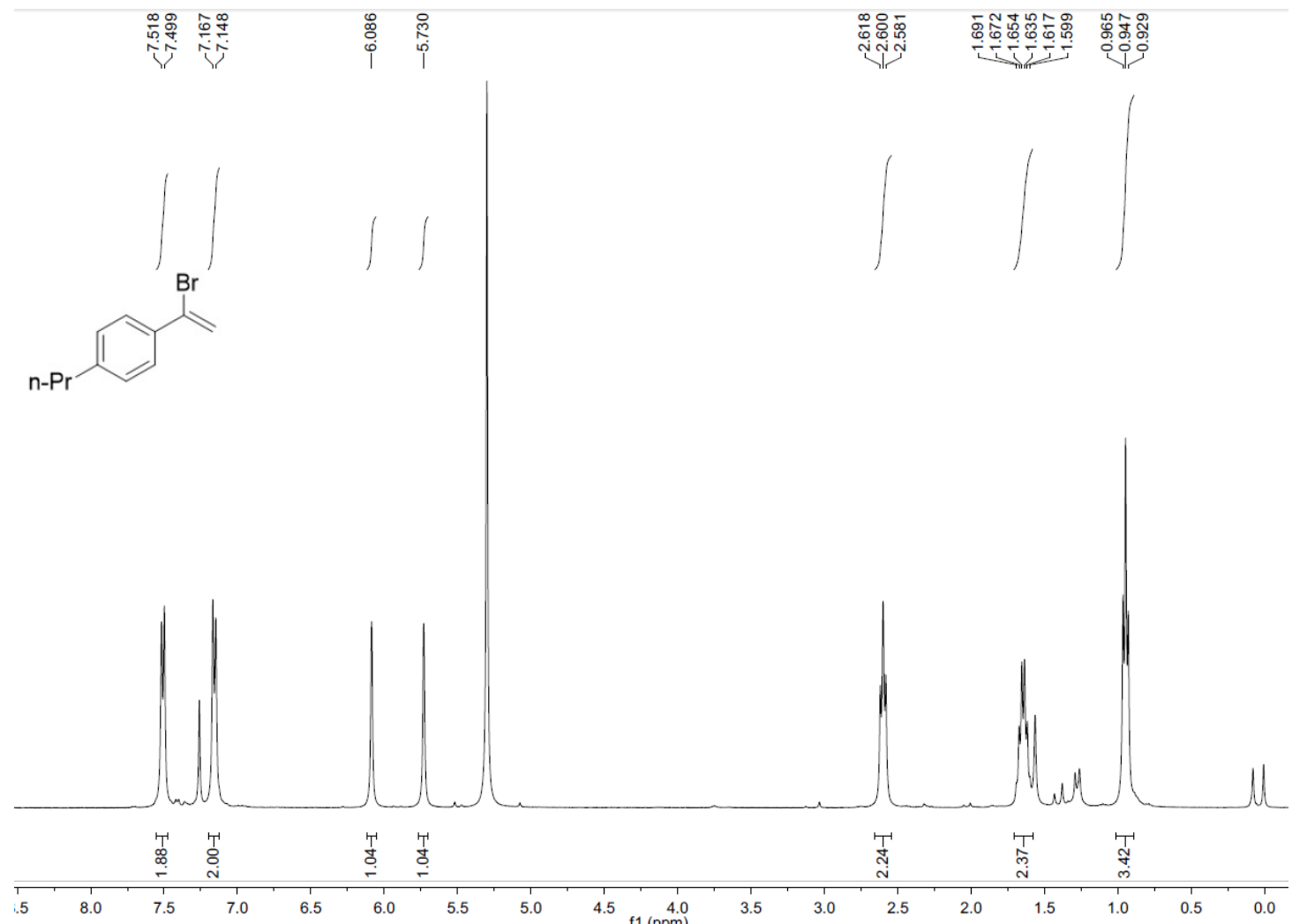

$2 \mathrm{i}(\mathrm{X}=\mathrm{Br})$ :

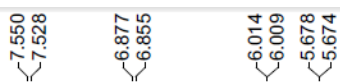

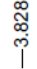

(c)
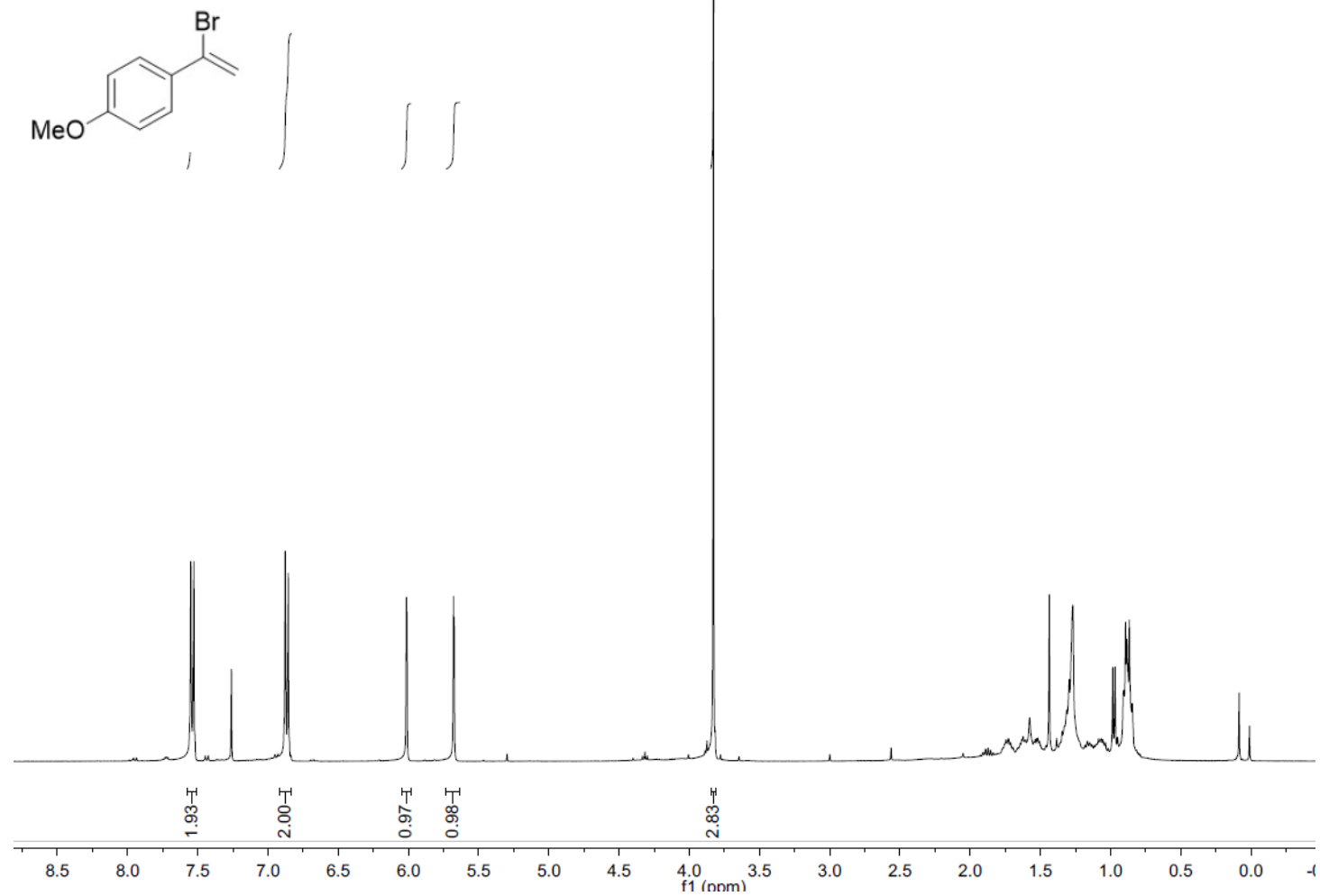


\section{$2 \mathrm{j}(\mathrm{X}=\mathrm{Br})$}

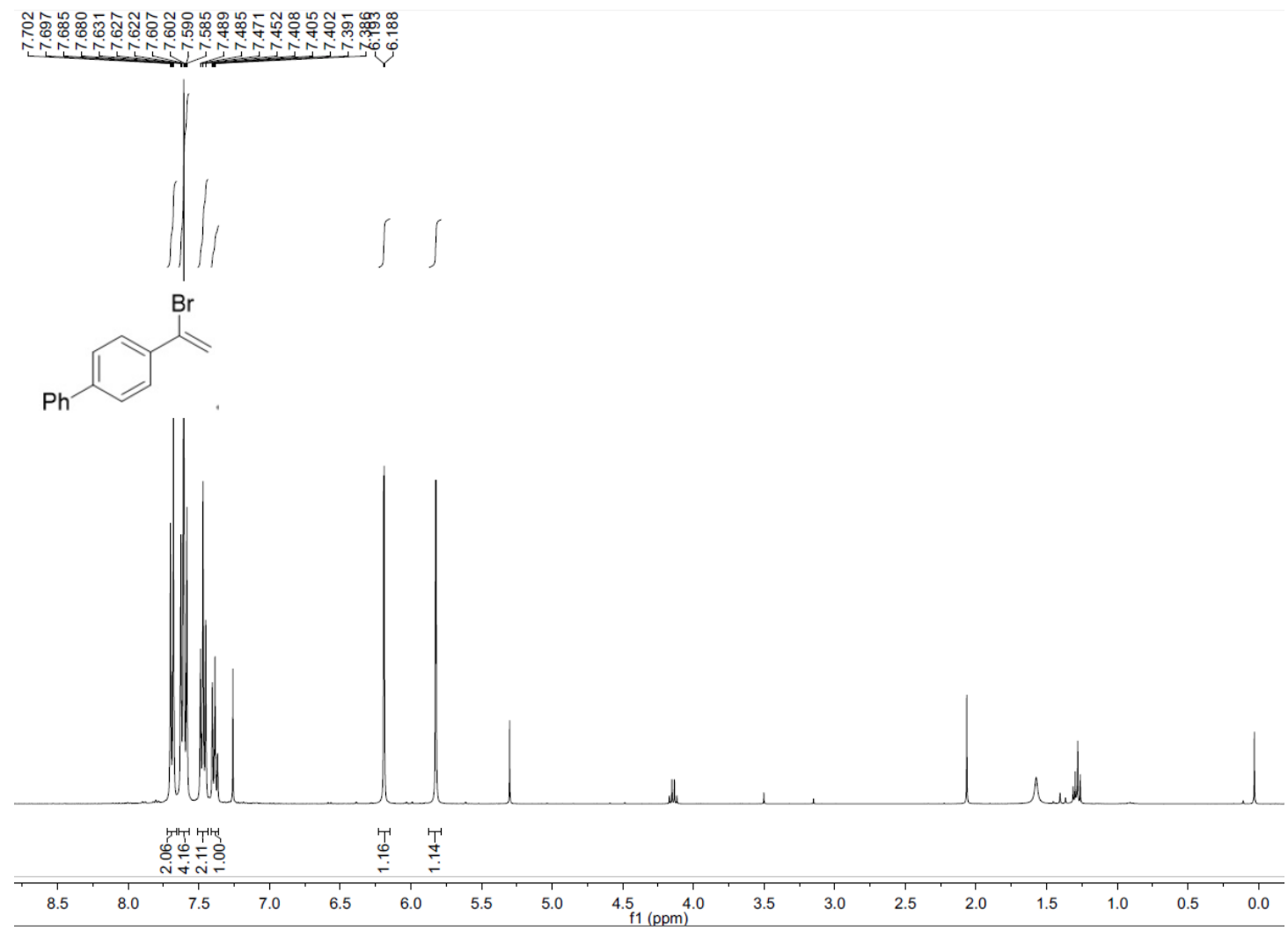

2k (X=Br):

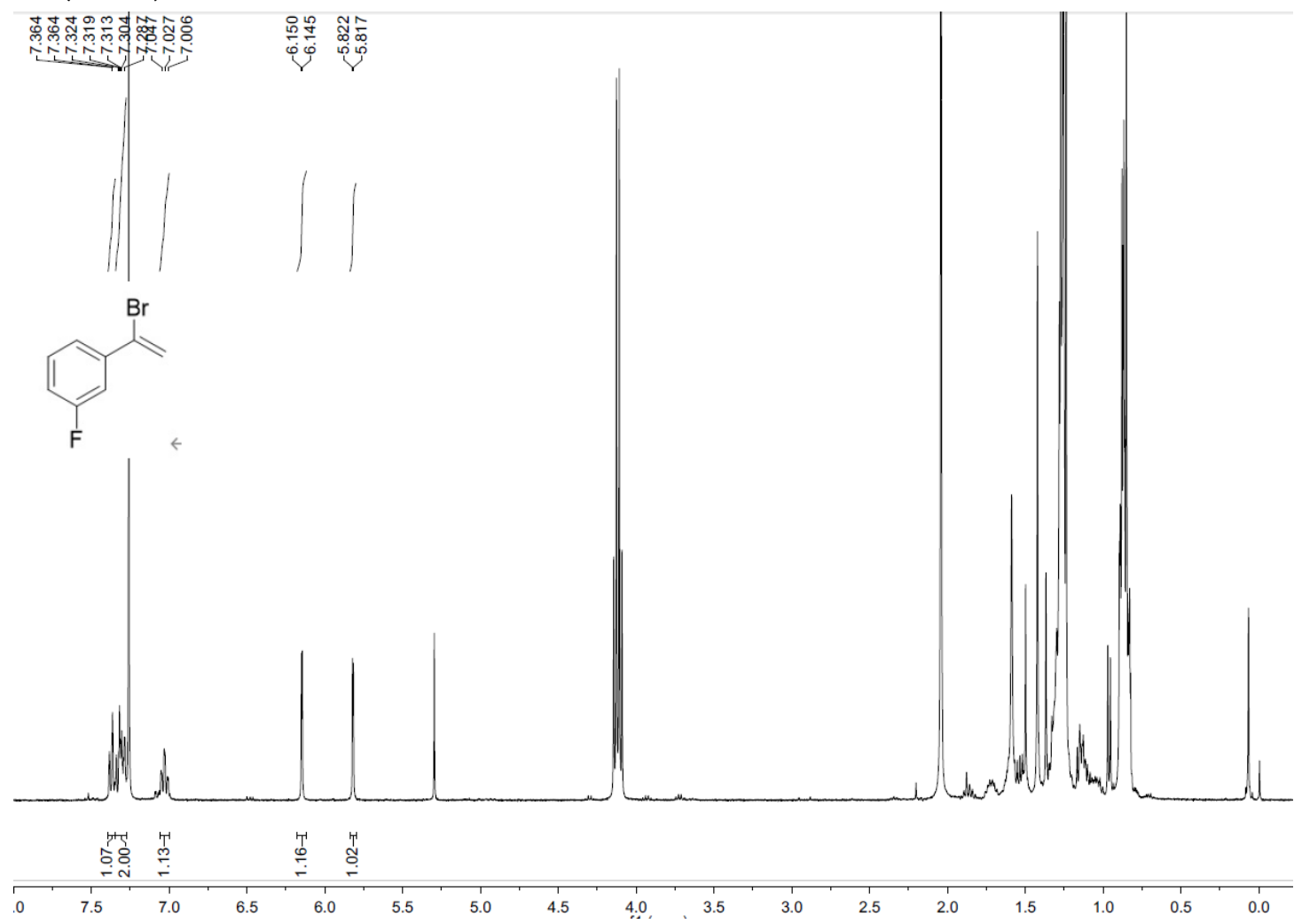


$21(\mathrm{X}=\mathrm{Br})$ :

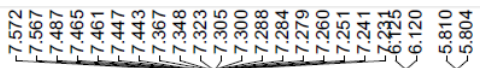

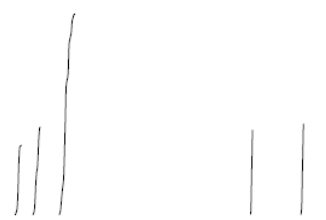

Cl

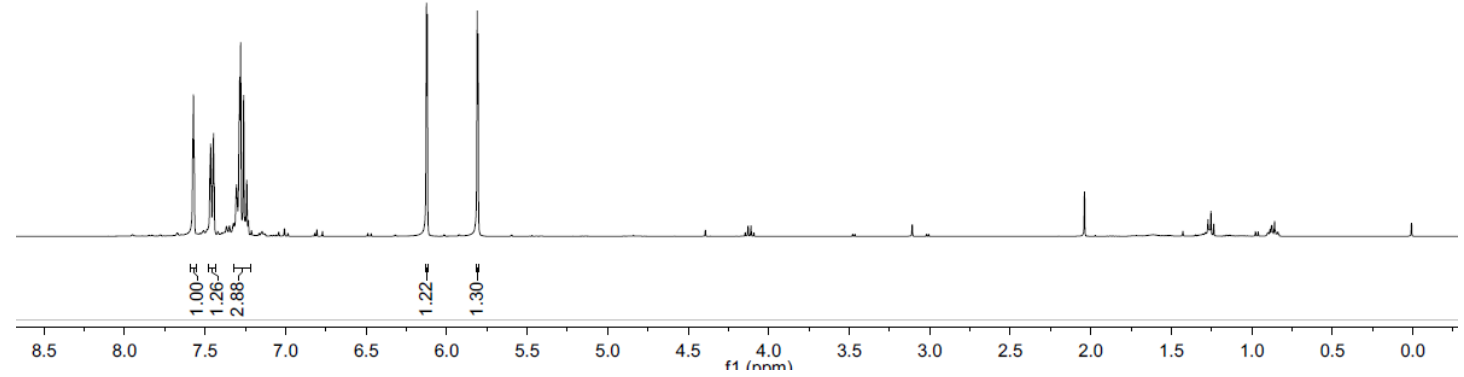

$2 \mathrm{~m}(\mathrm{X}=\mathrm{Br})$ :

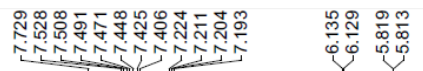

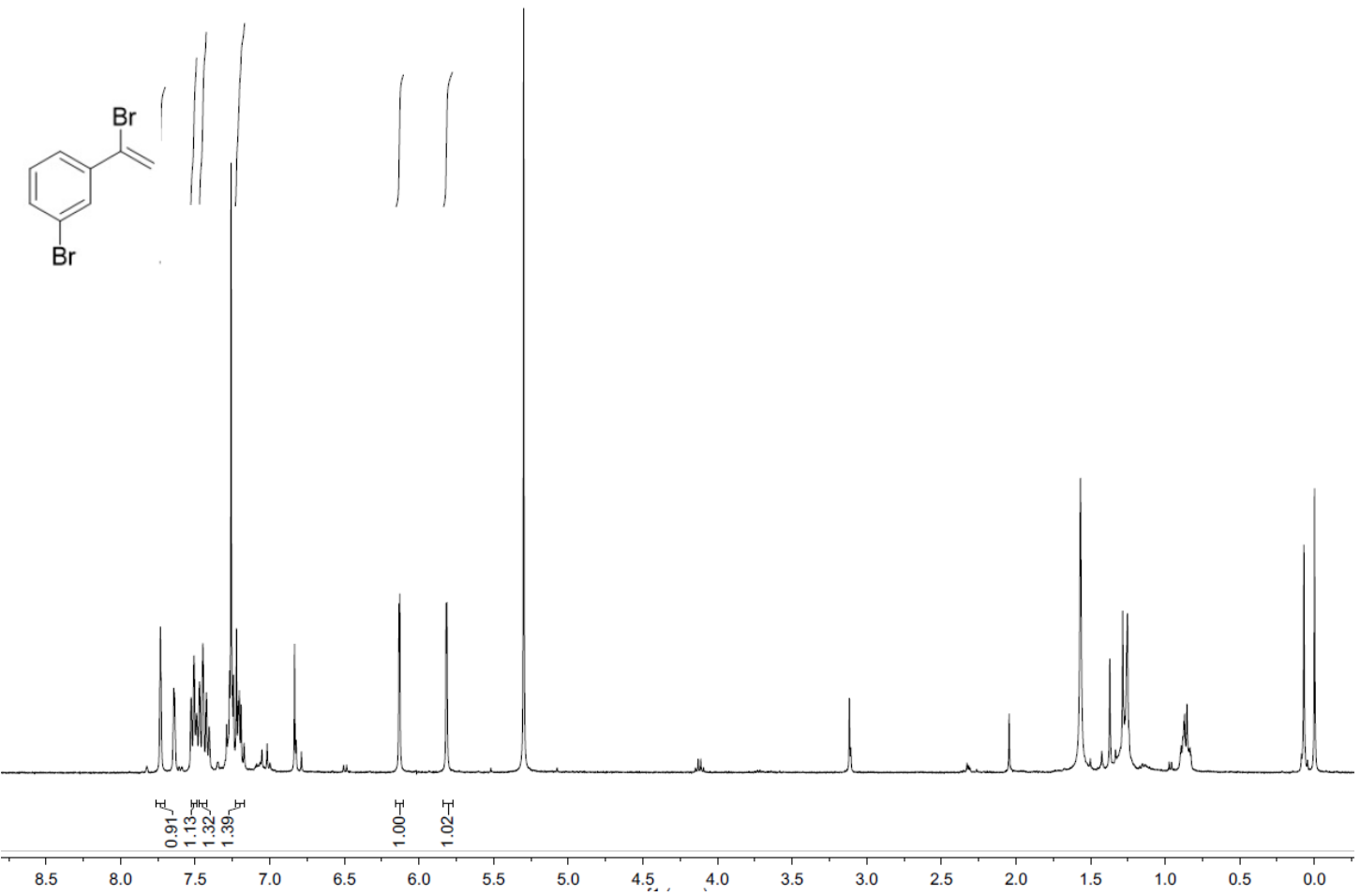


2n ( $\mathrm{X}=\mathrm{Br})$ :

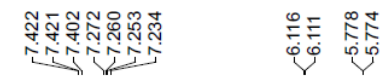<smiles>C#CC(=C)c1cccc(C)c1</smiles>
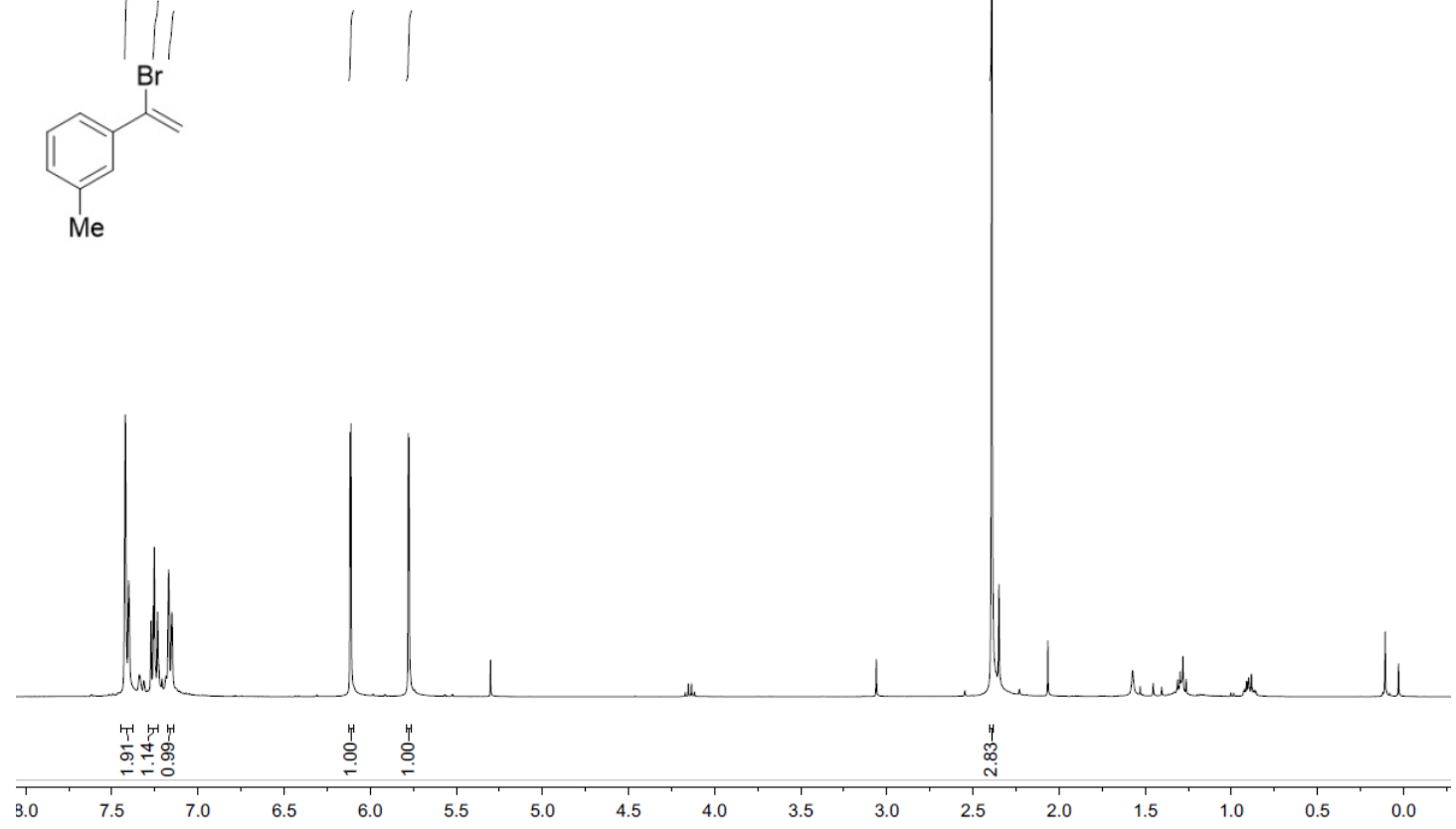

$20(\mathrm{X}=\mathrm{Br})$ :

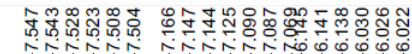

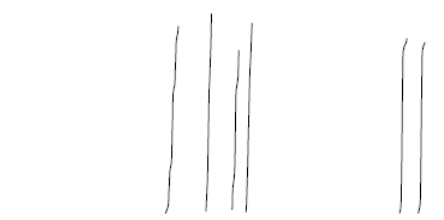<smiles>C=C(Br)c1ccccc1F</smiles>

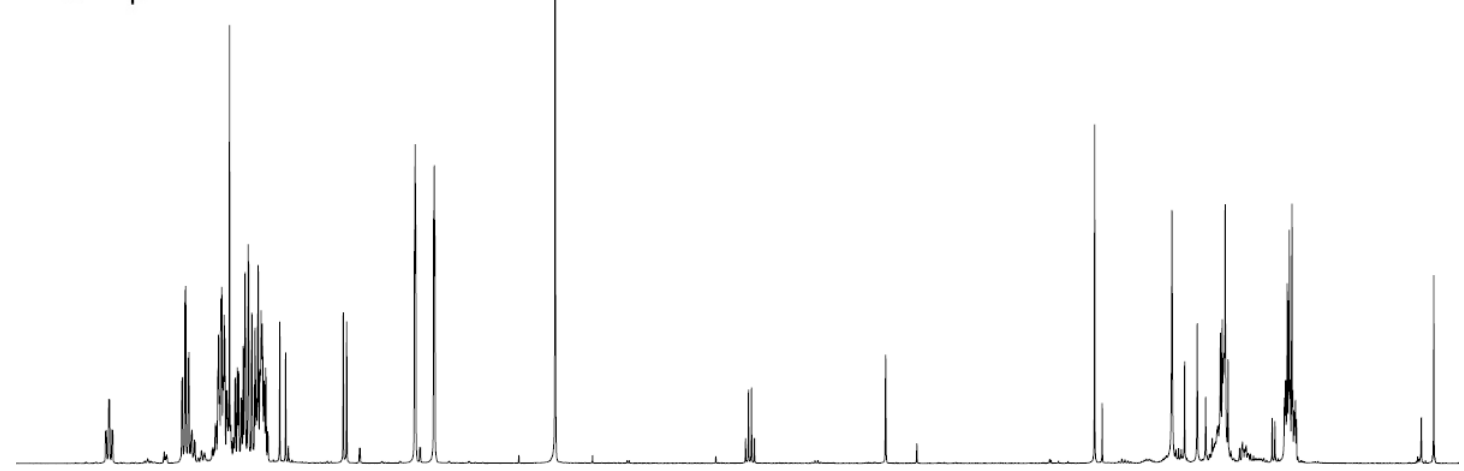

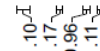

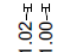

\begin{tabular}{llllll}
\hline & 1 & 1 & 1 & 1 & 1 \\
3.5 & 8.0 & 7.5 & 7.0 & 6.5 & 6.0
\end{tabular} $\begin{array}{lllllll}5.5 & 5.0 & 4.5 & \ldots & 4.0 & 3.5\end{array}$ 
$2 p(X=B r):$

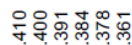

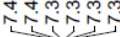

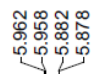

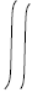

$\overbrace{C l}^{\mathrm{Br}}$
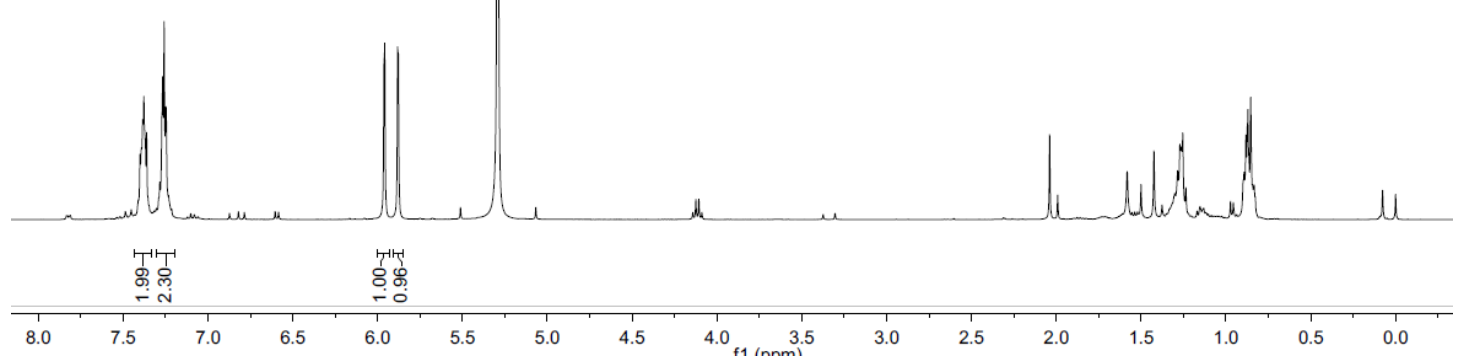

$\mathbf{2 q}(\mathrm{X}=\mathrm{Br})$ :

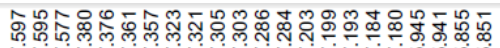

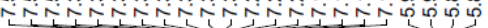
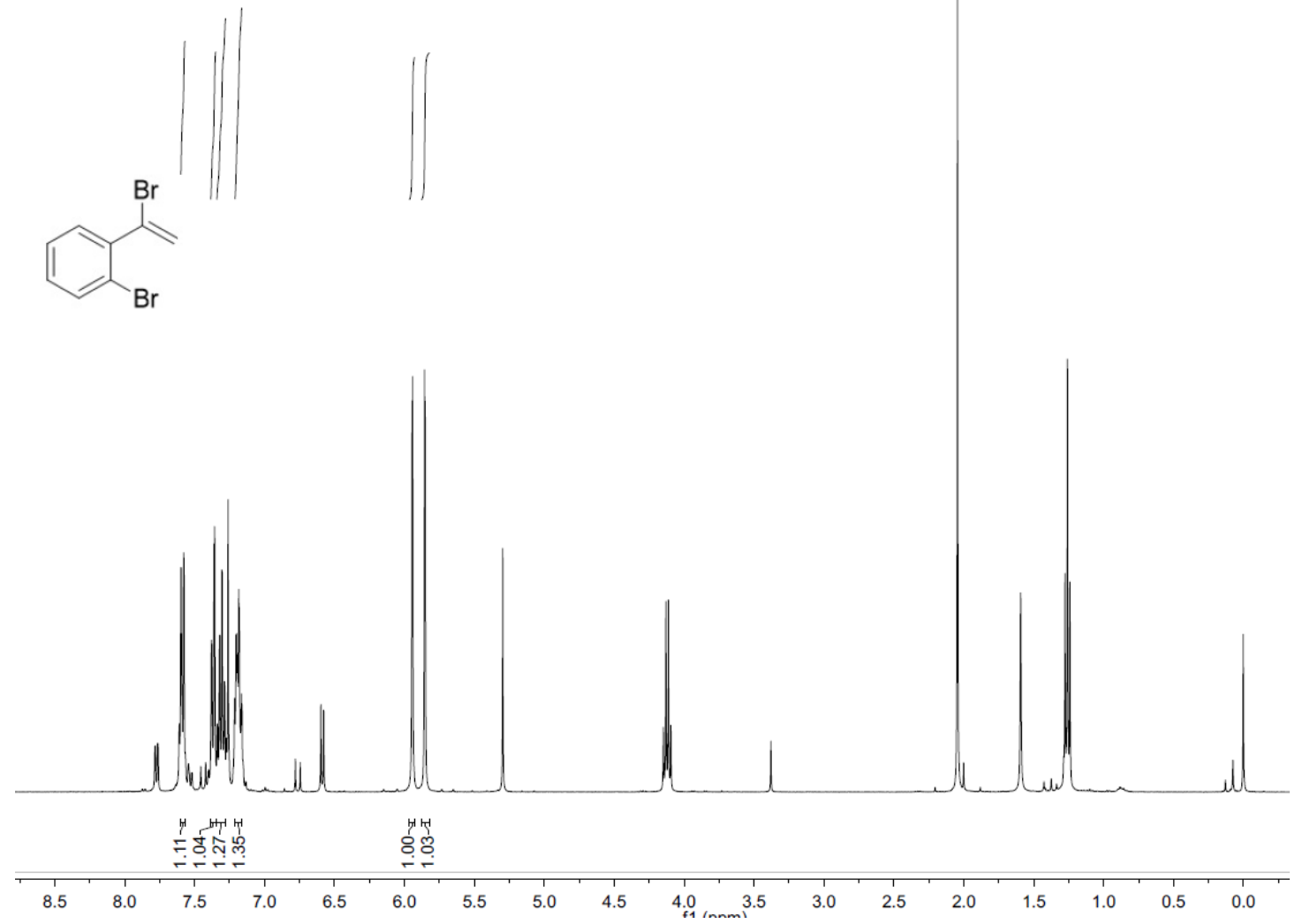
2r $(\mathrm{X}=\mathrm{Br})$ :

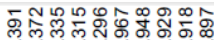

NNNN00000

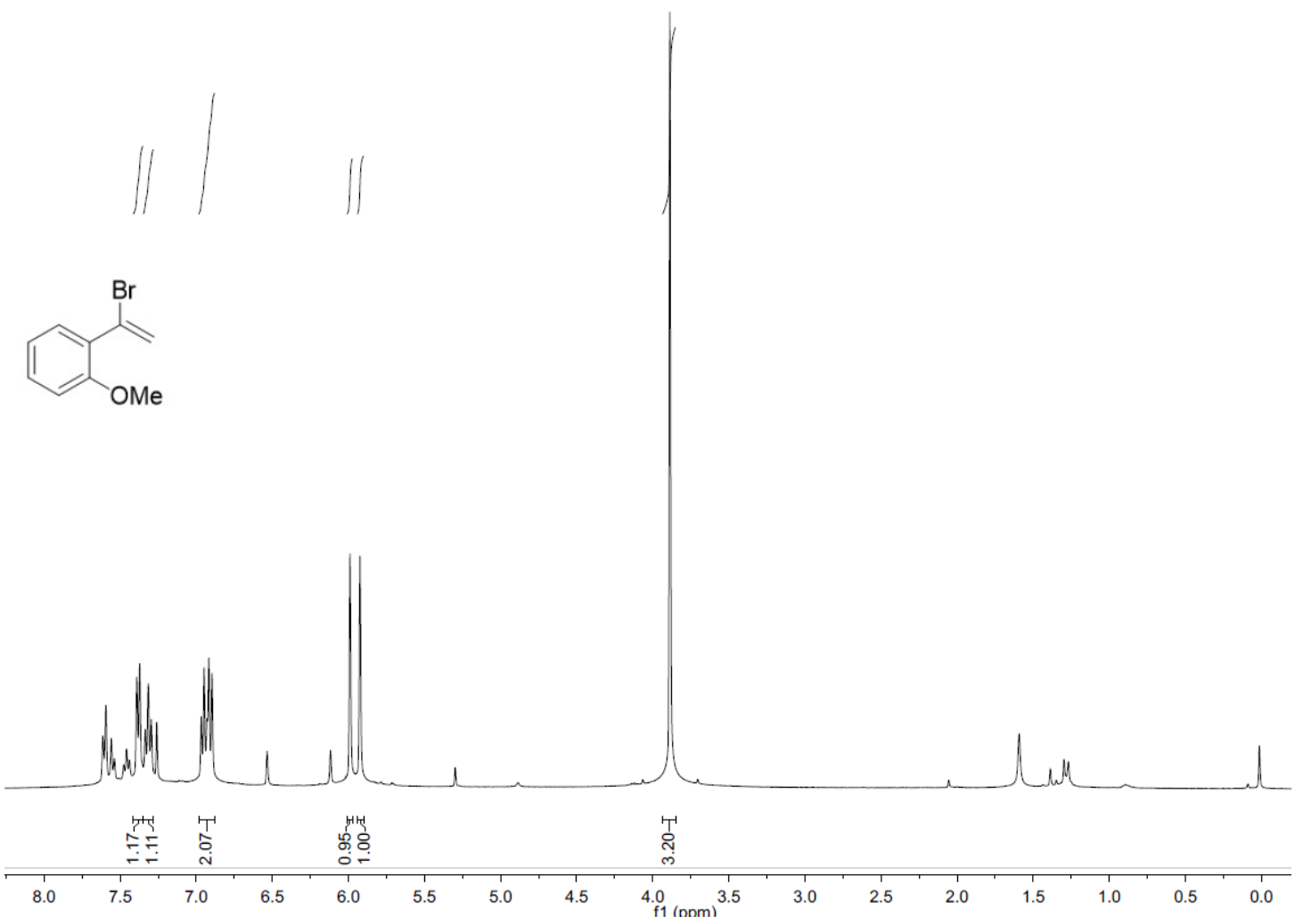

2s $(\mathrm{X}=\mathrm{Br})$ :

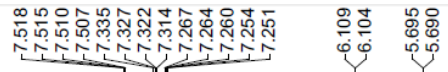

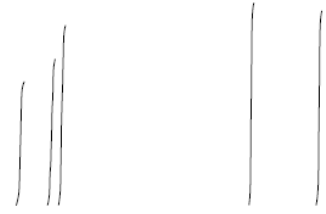

S1

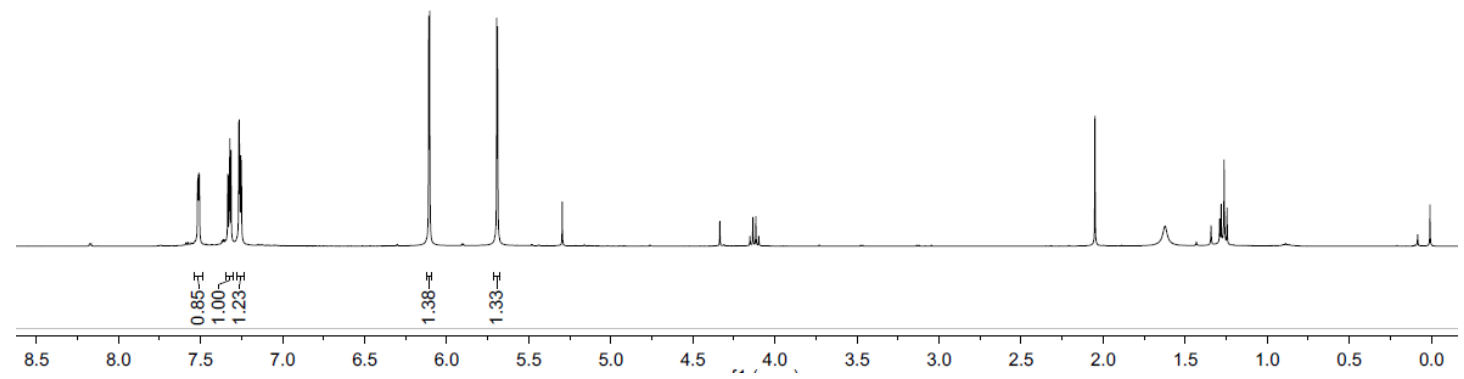


2a $(X=I)$ :

$\begin{array}{lll}0 & 0 \\ 0 & 0 \\ 0\end{array}$
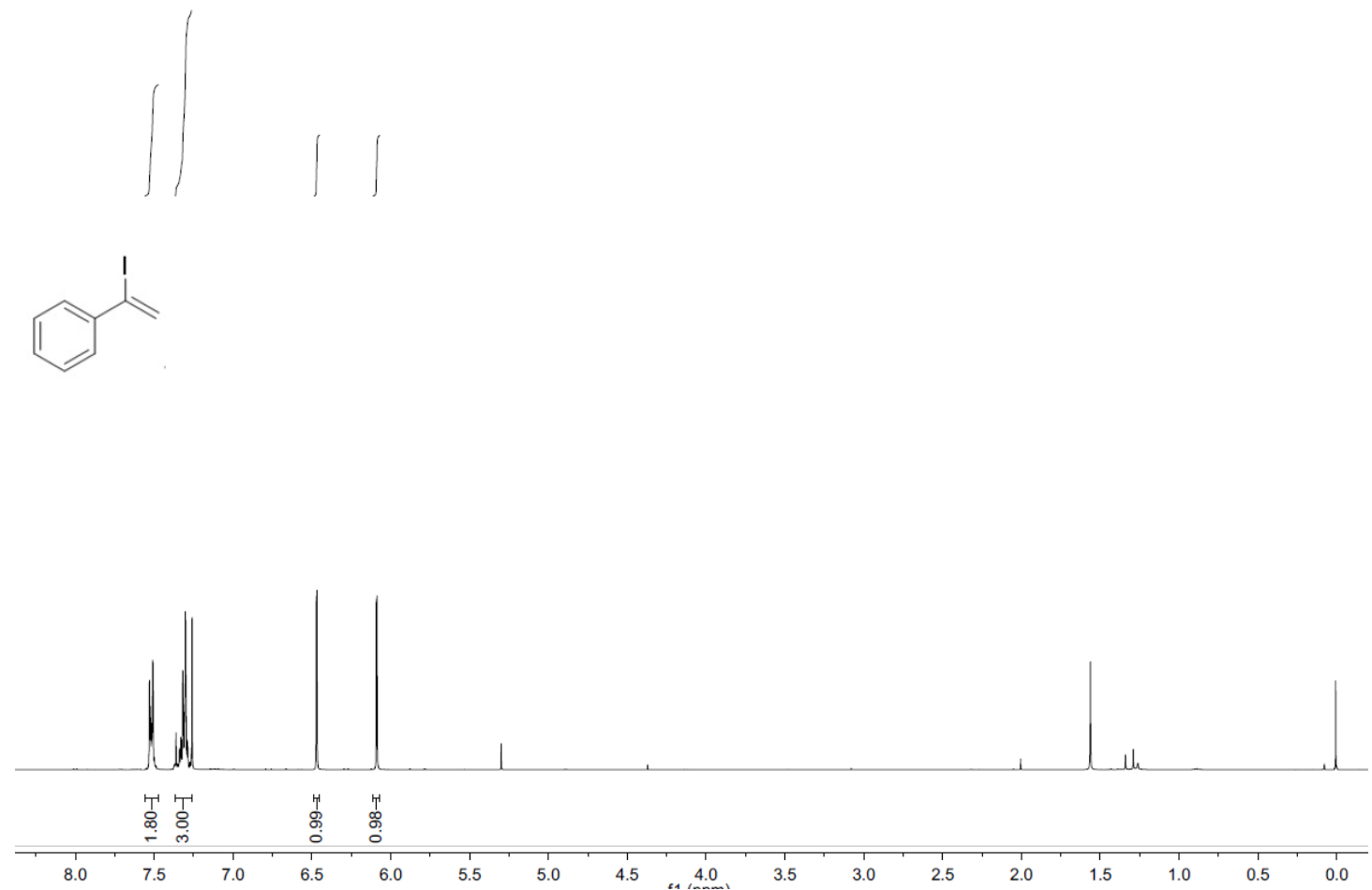

$2 b(X=I)$ :

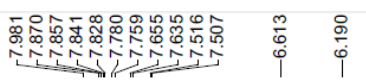
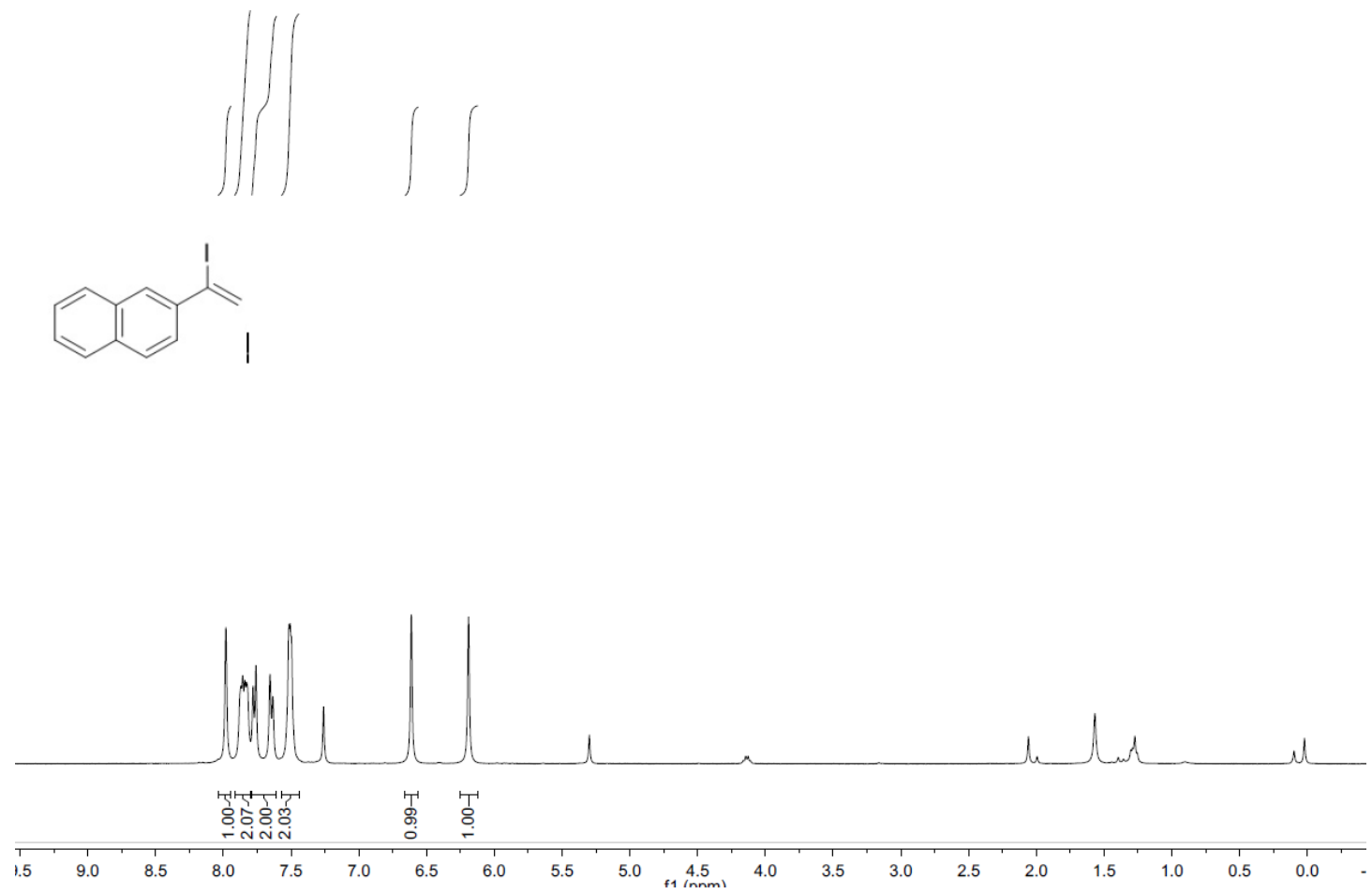
2c $(\mathrm{X}=\mathrm{I})$ :

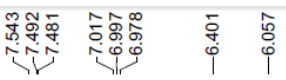
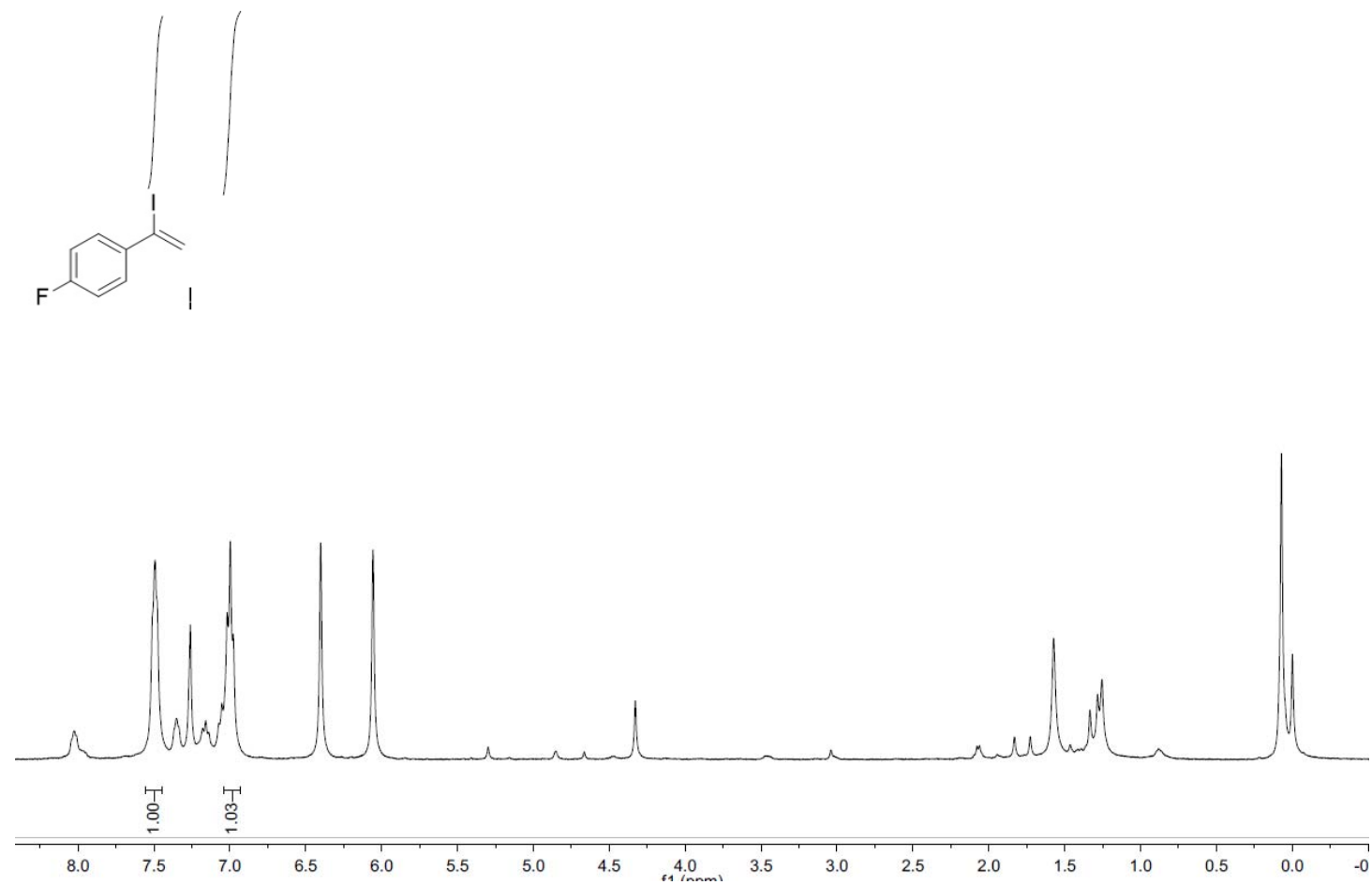

2d (X=I):

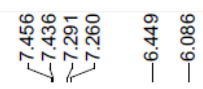

(1)
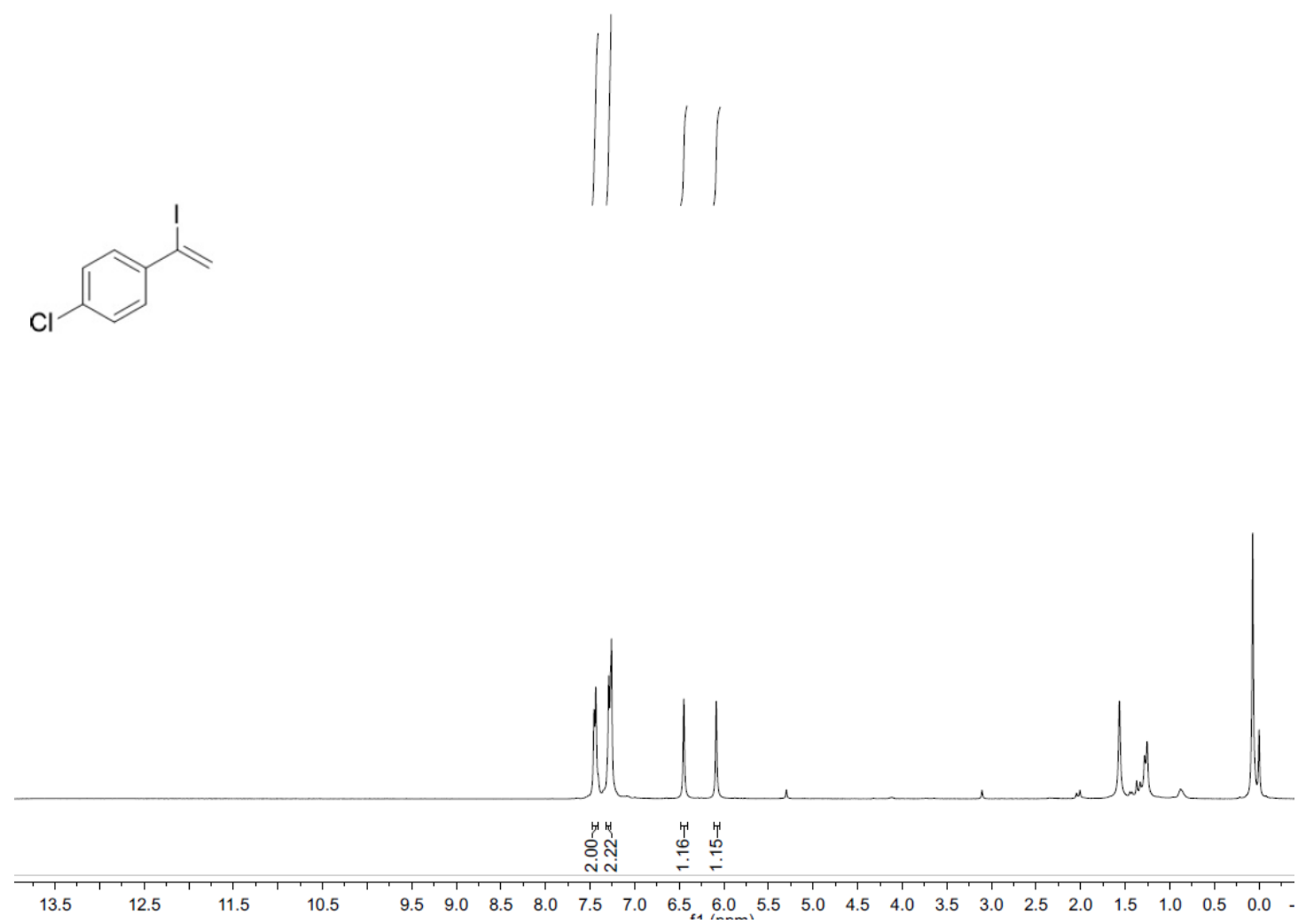
2e $(X=I)$ :

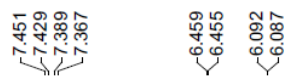
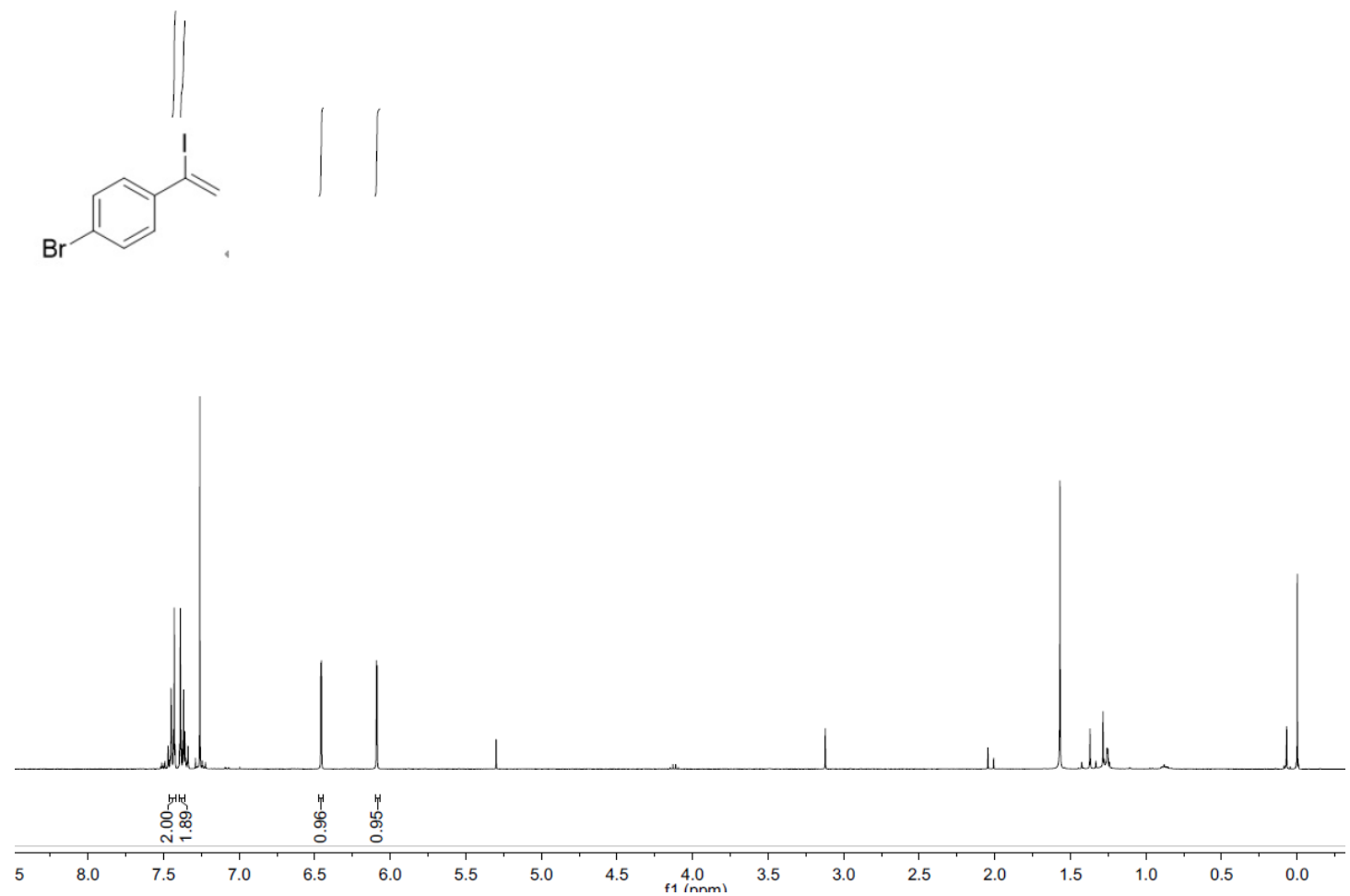
$2 f(X=I)$ :

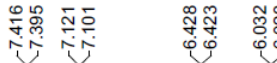
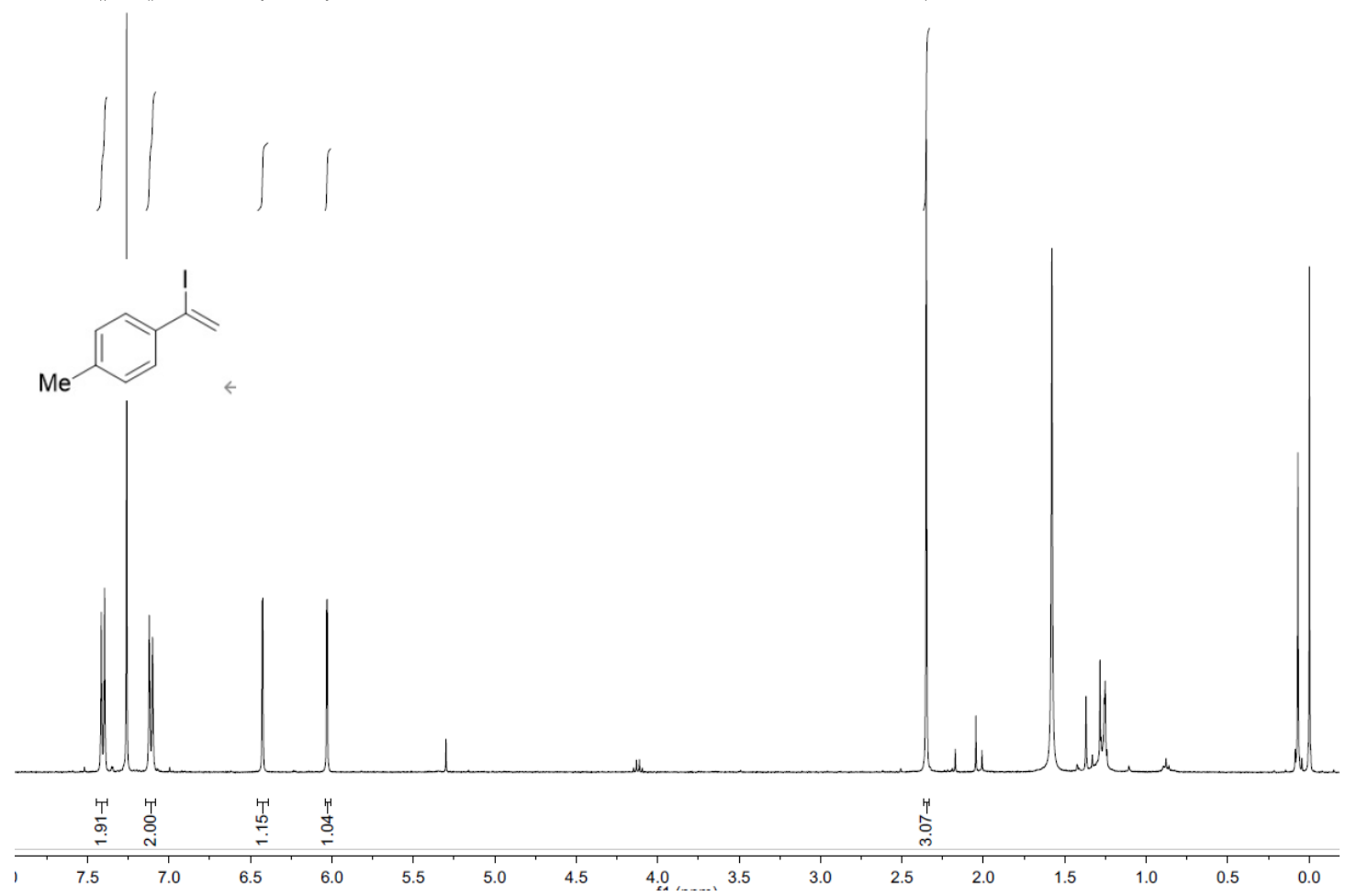
$2 \mathrm{~g}(\mathrm{X}=\mathrm{I})$ :

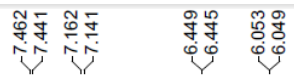

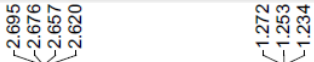

(1)
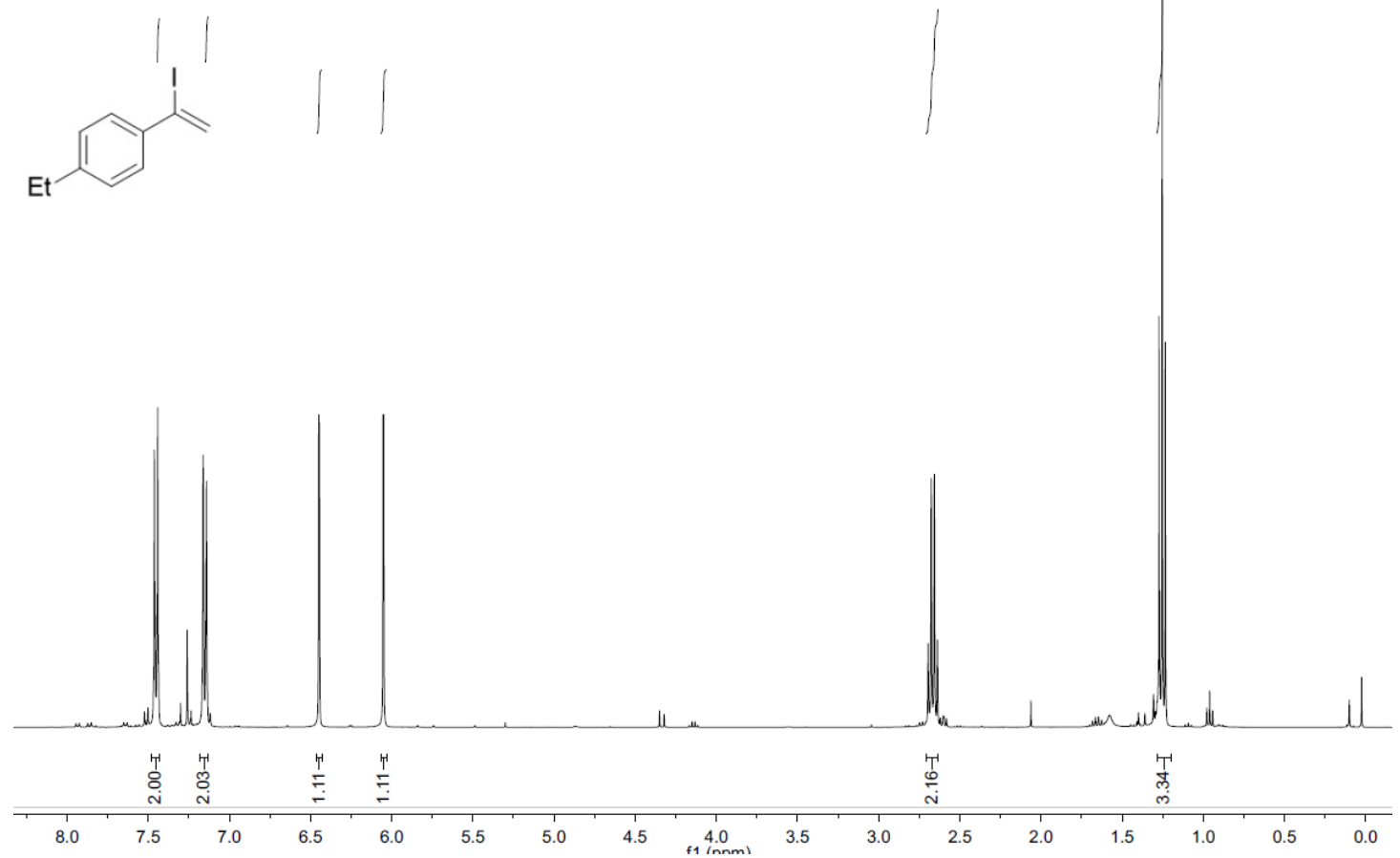

2h (X=I):

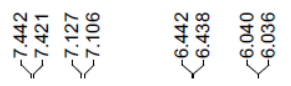

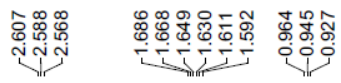
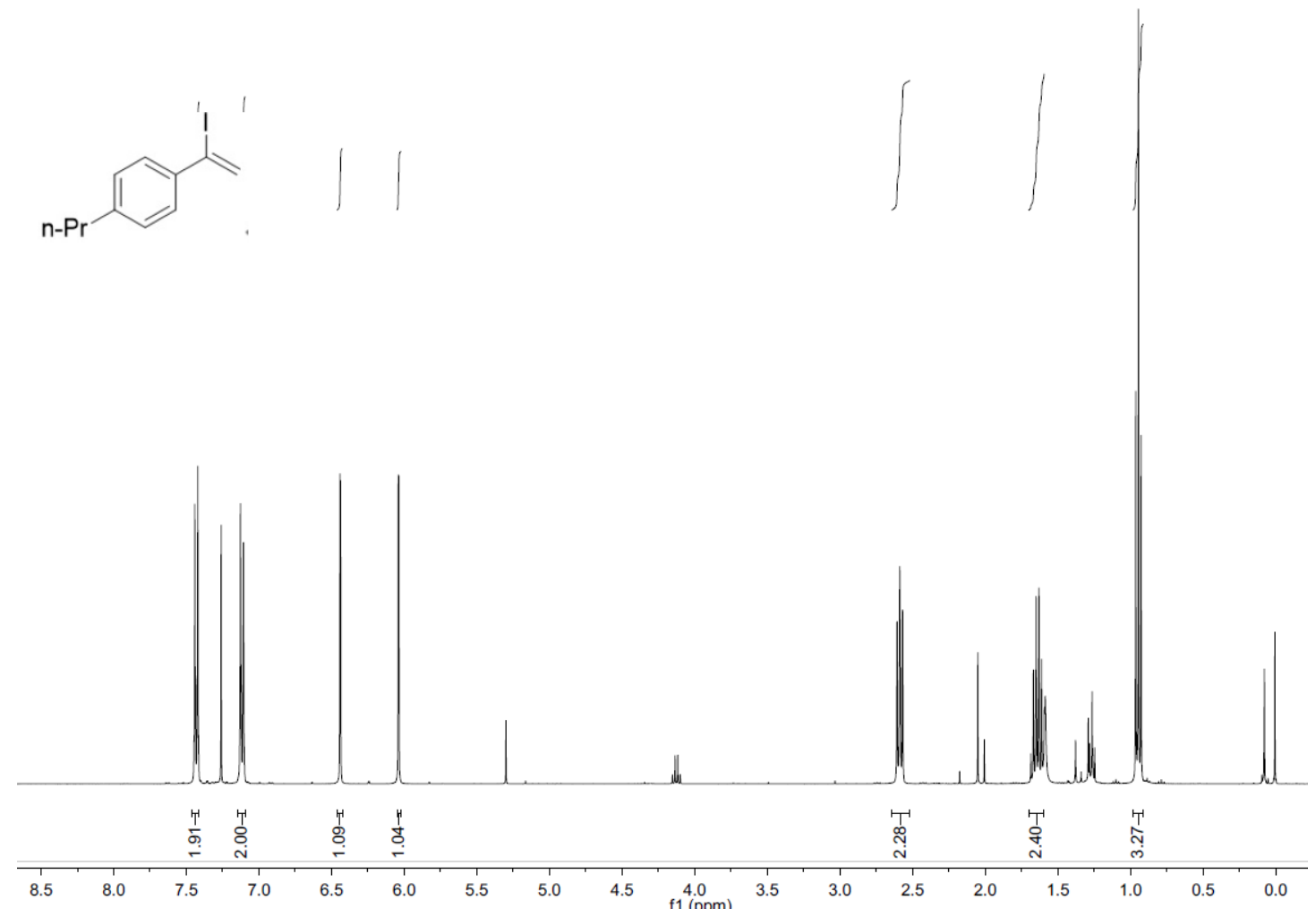
$2 i(X=I)$ :

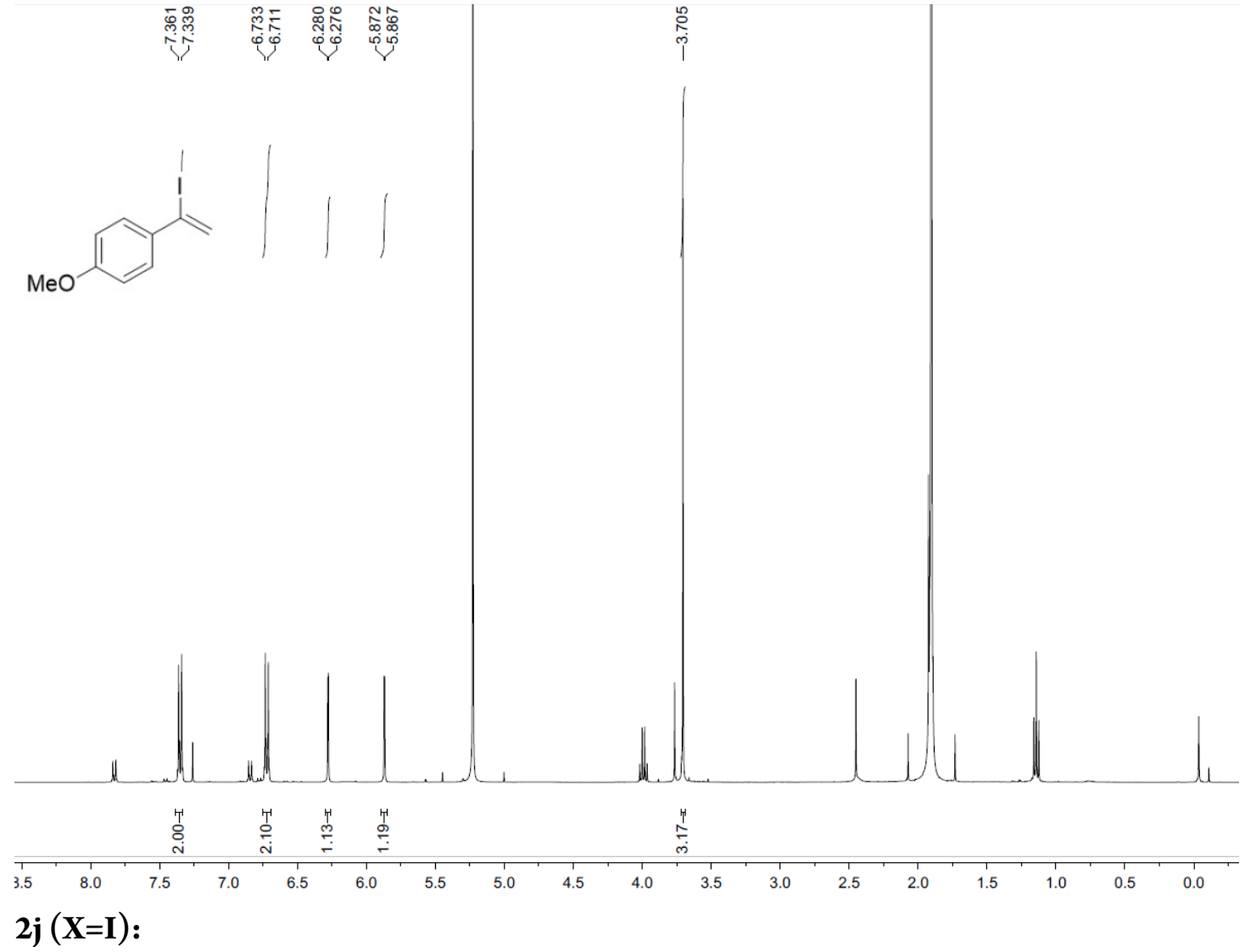

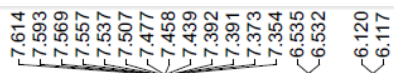
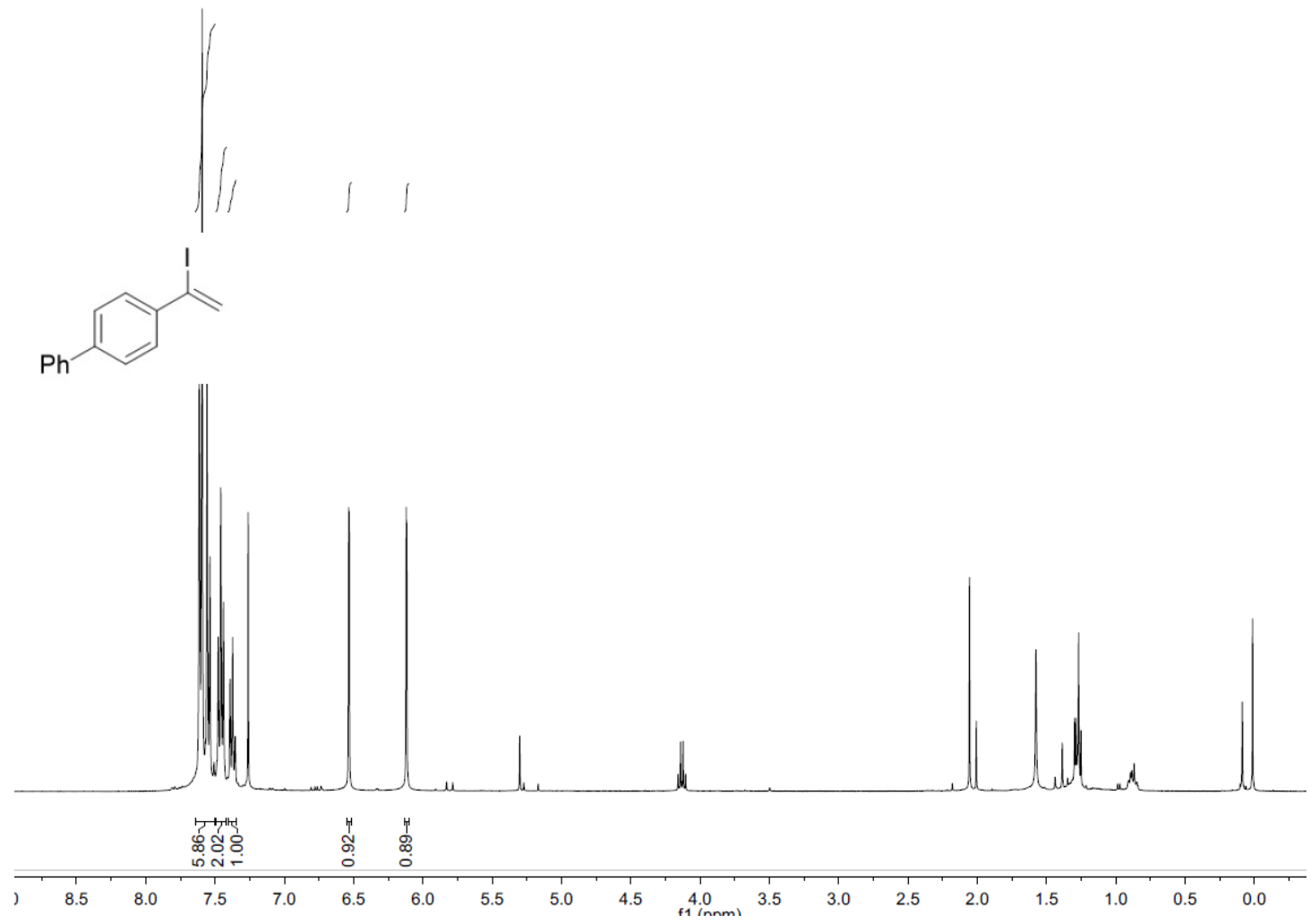
$2 k(X=I)$ :

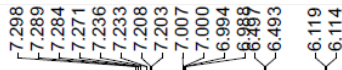

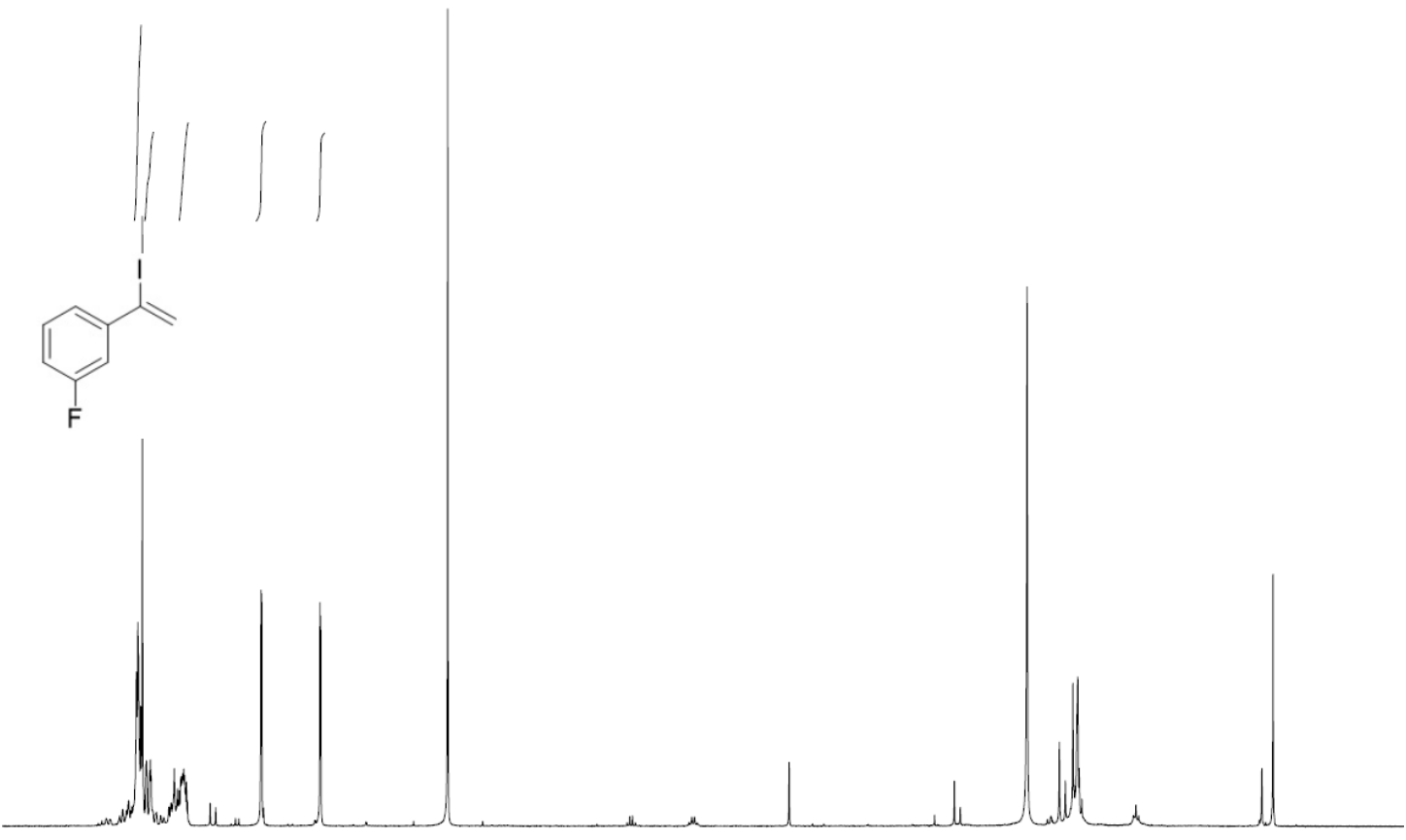

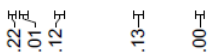

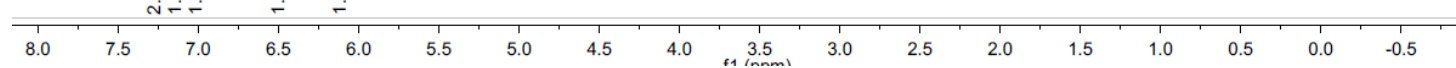

$21(X=I)$ :

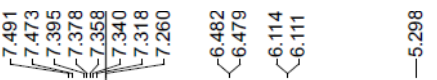

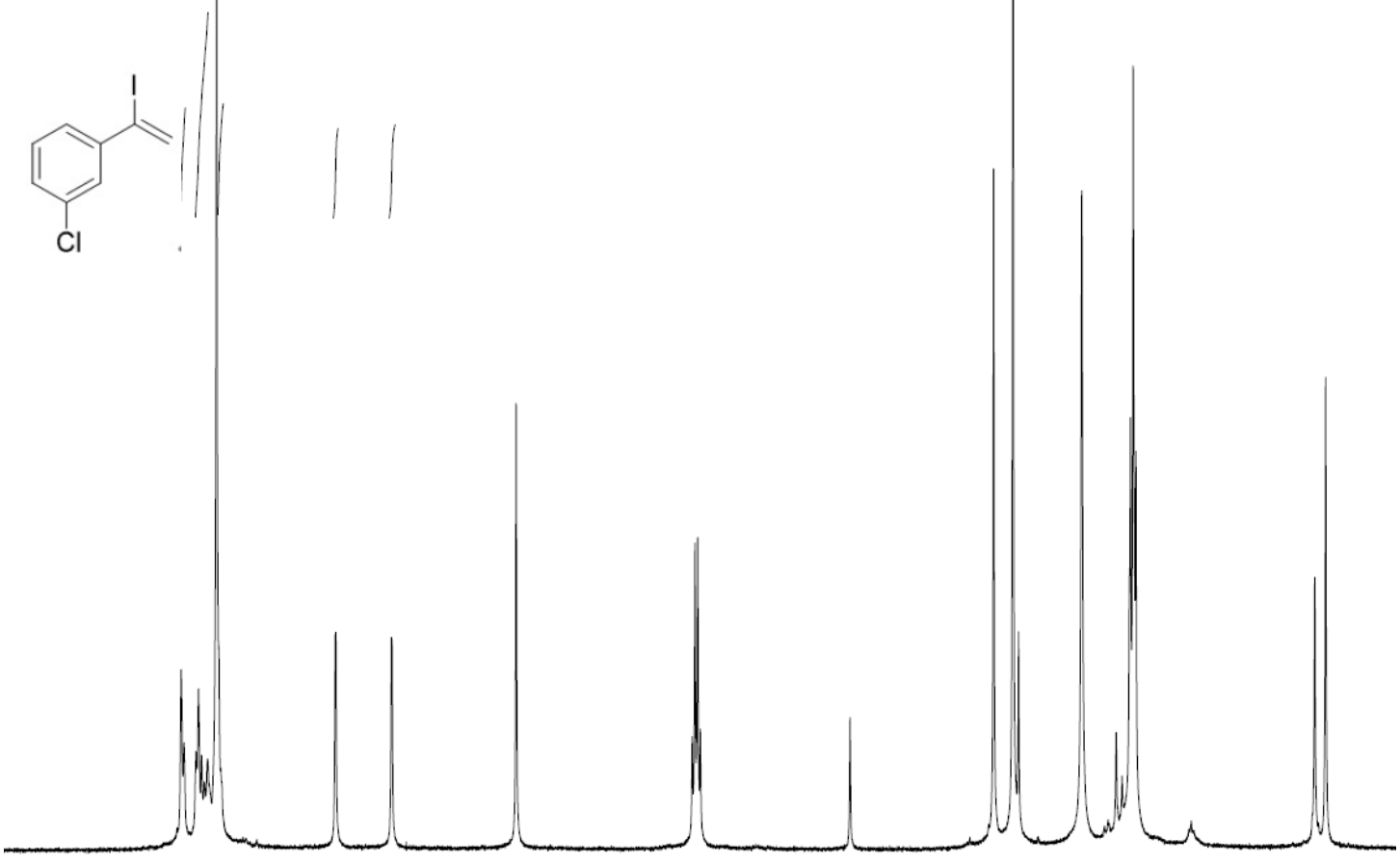

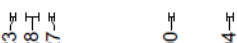

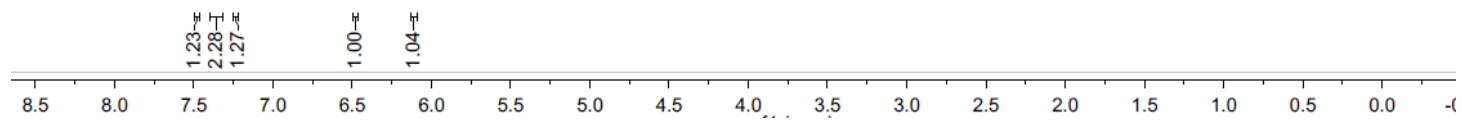




\section{$2 \mathrm{~m}(\mathrm{X}=\mathrm{I})$ :}

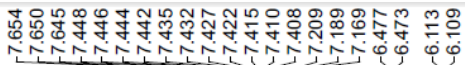

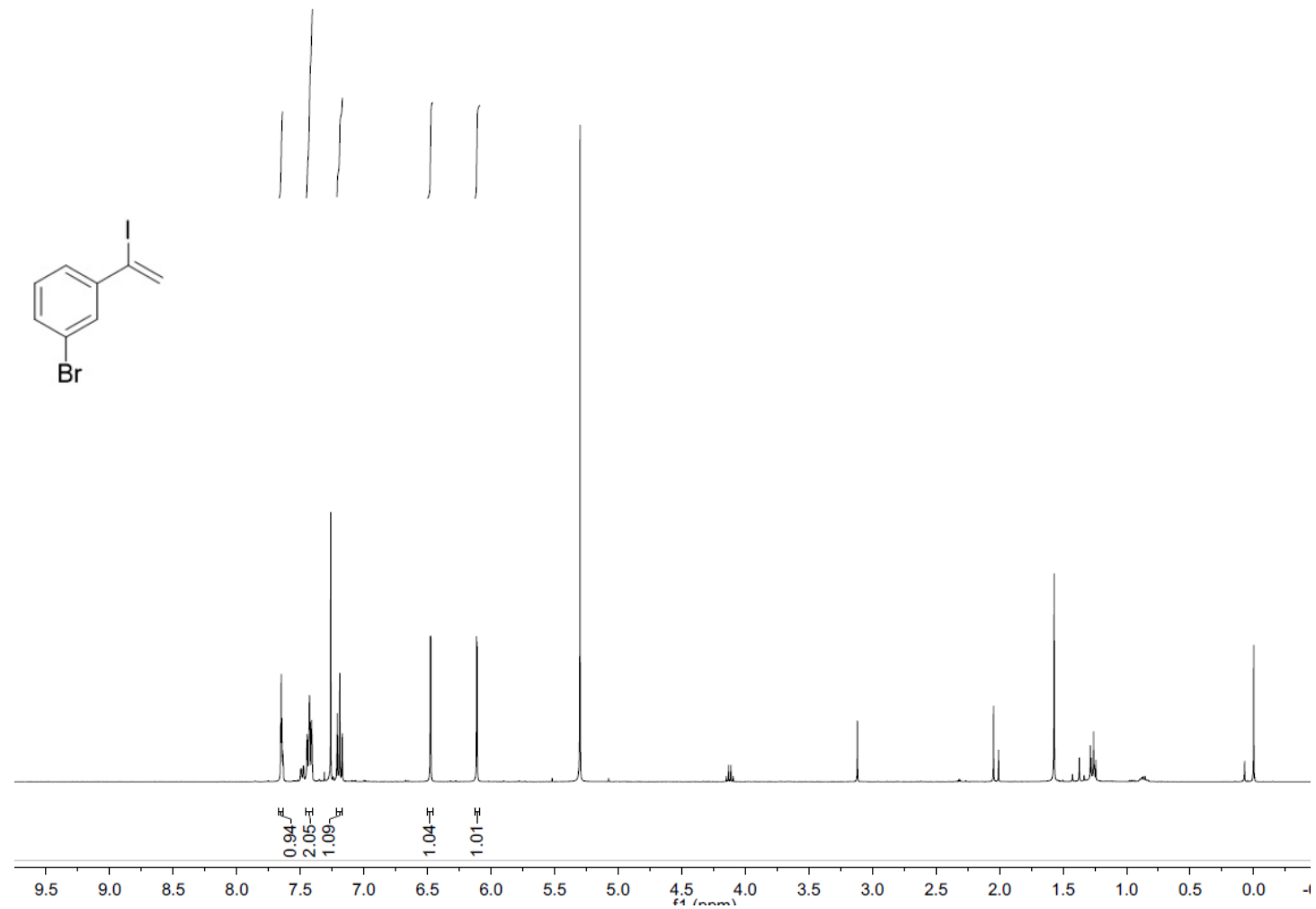

2n $(X=I)$ :

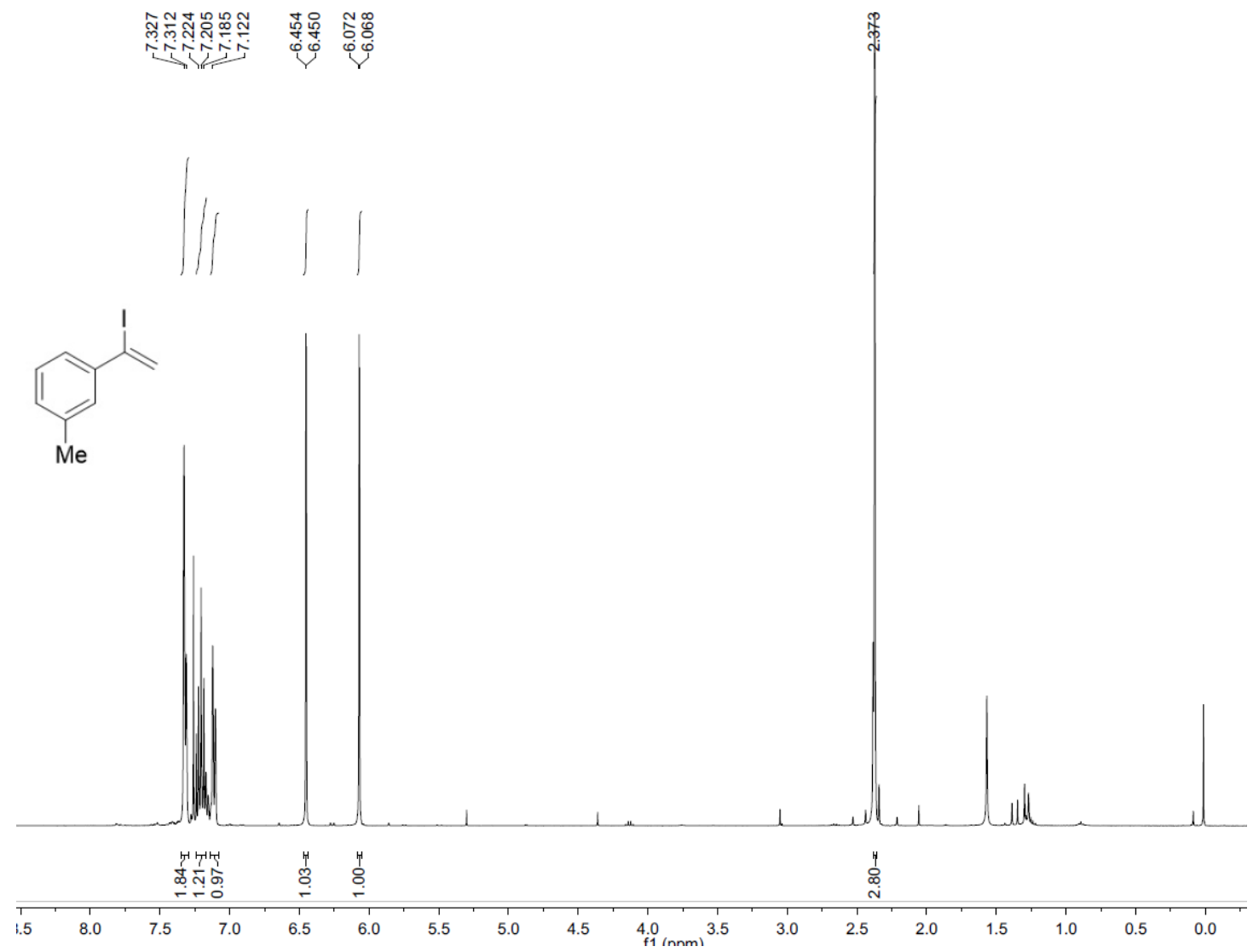


2o $(\mathrm{X}=\mathrm{I})$ :

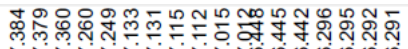

rititititiolog

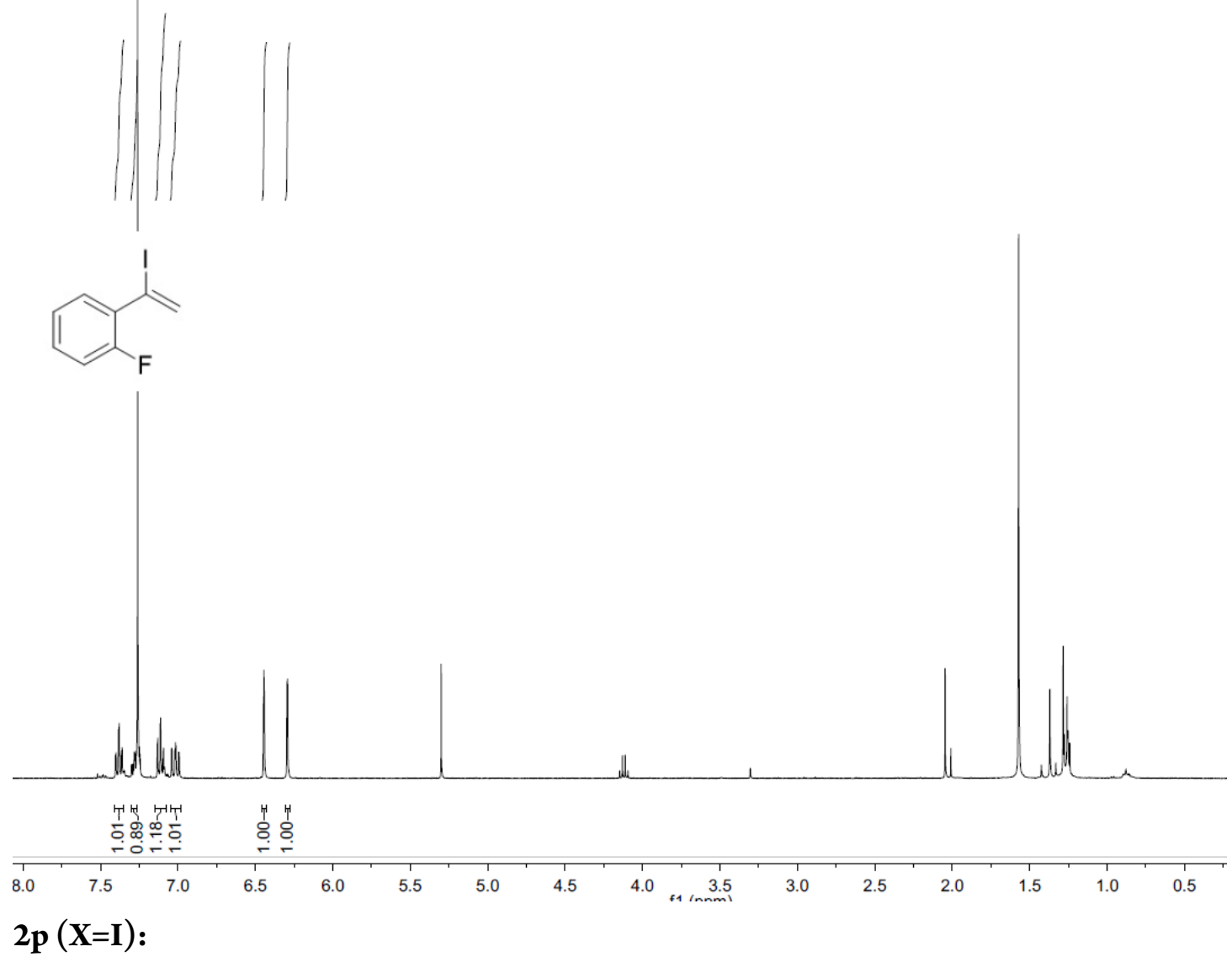

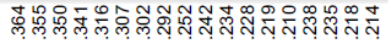

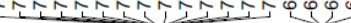
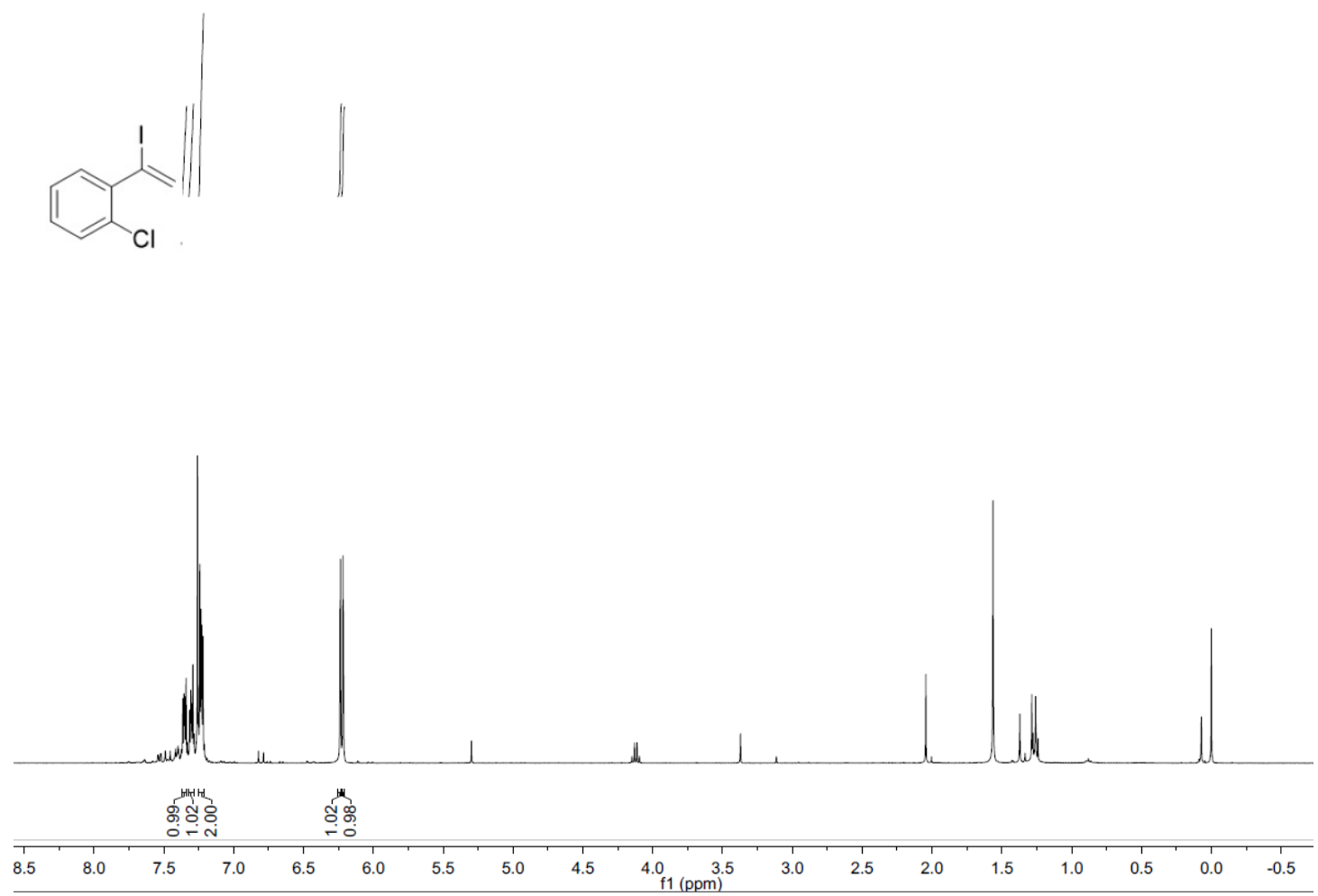


\section{2q $(X=I):$}

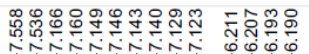
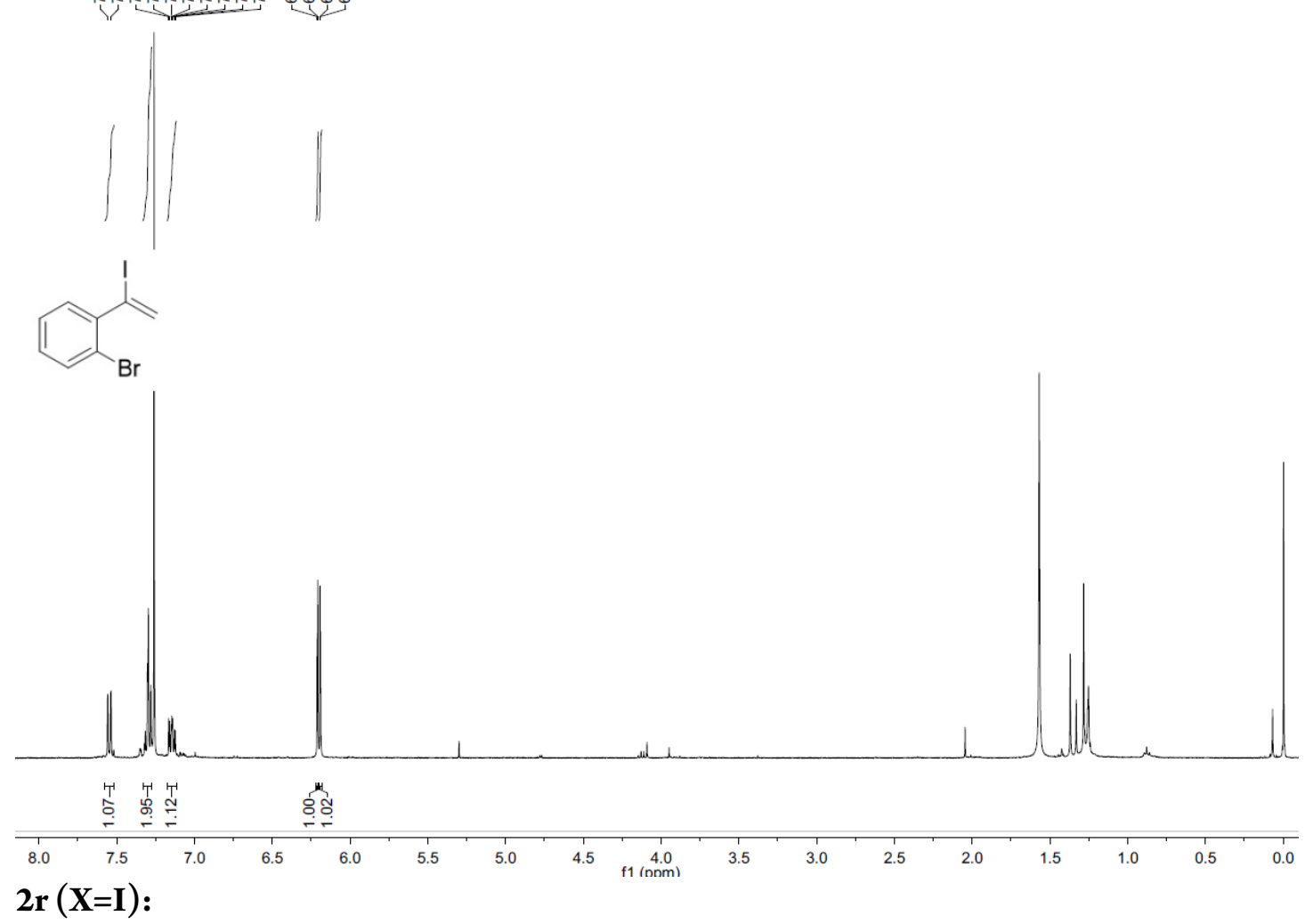

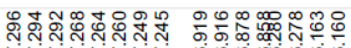

年
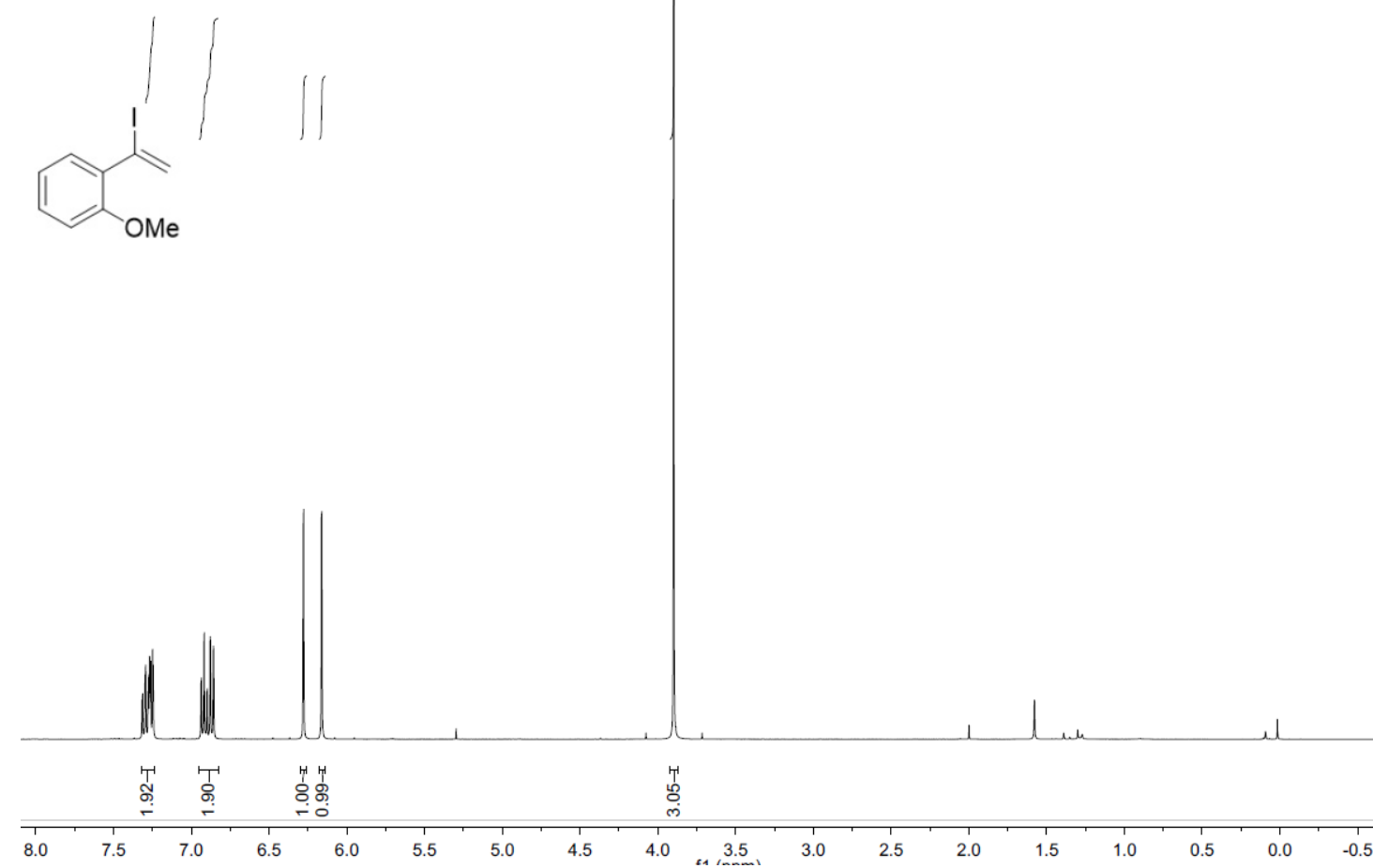
1B:

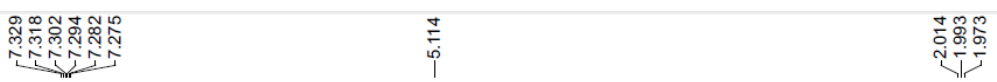

$\mathrm{HS}$

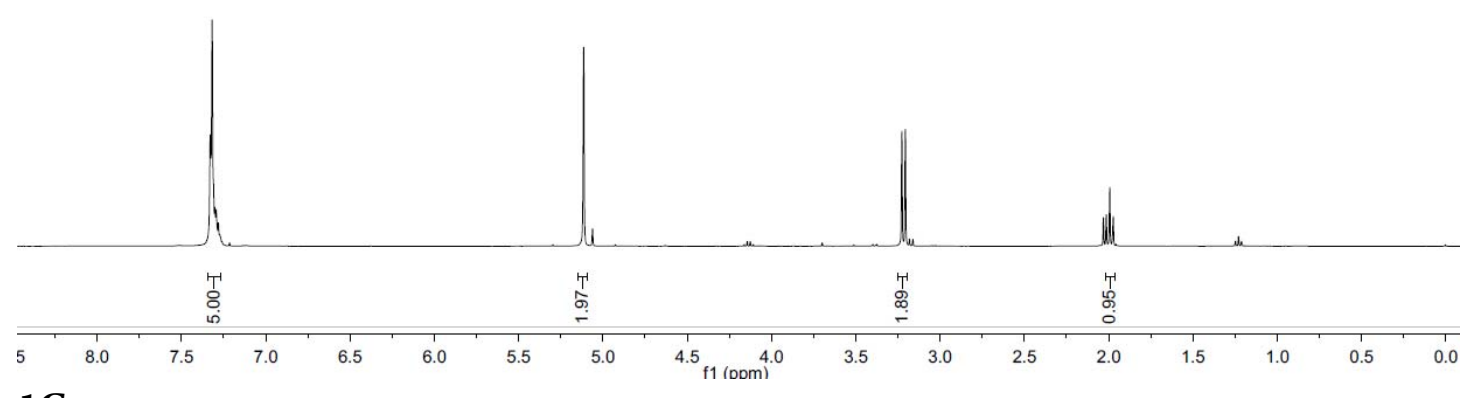

1C:

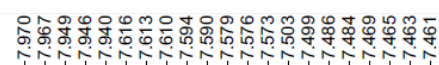
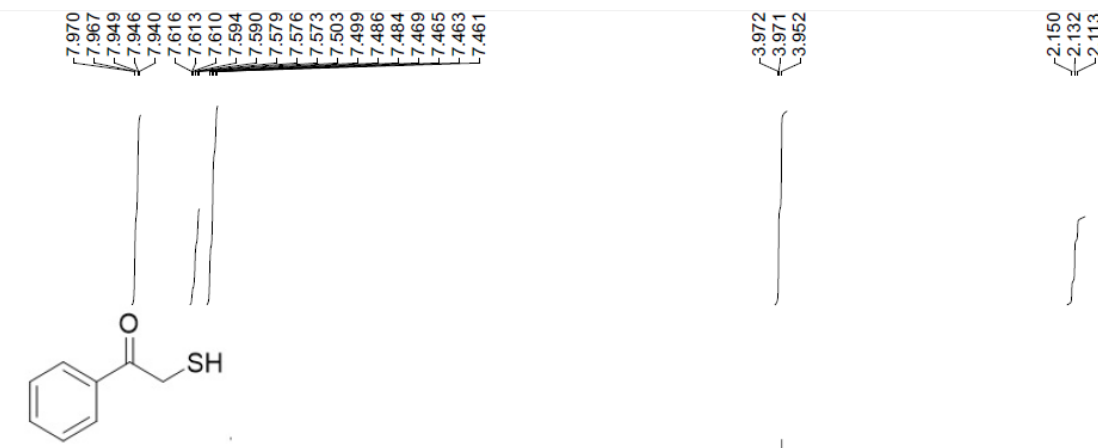

$\frac{\text { nin }}{\mathrm{n}}$

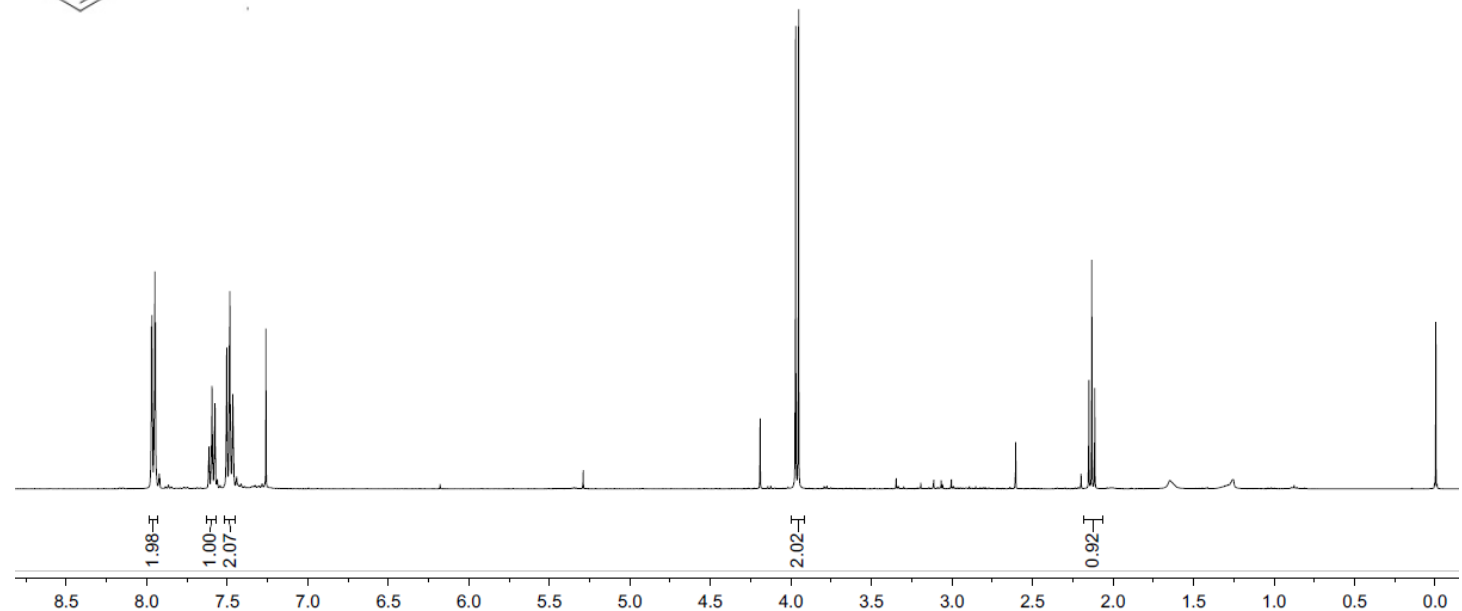


1D:

خัy

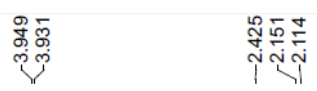
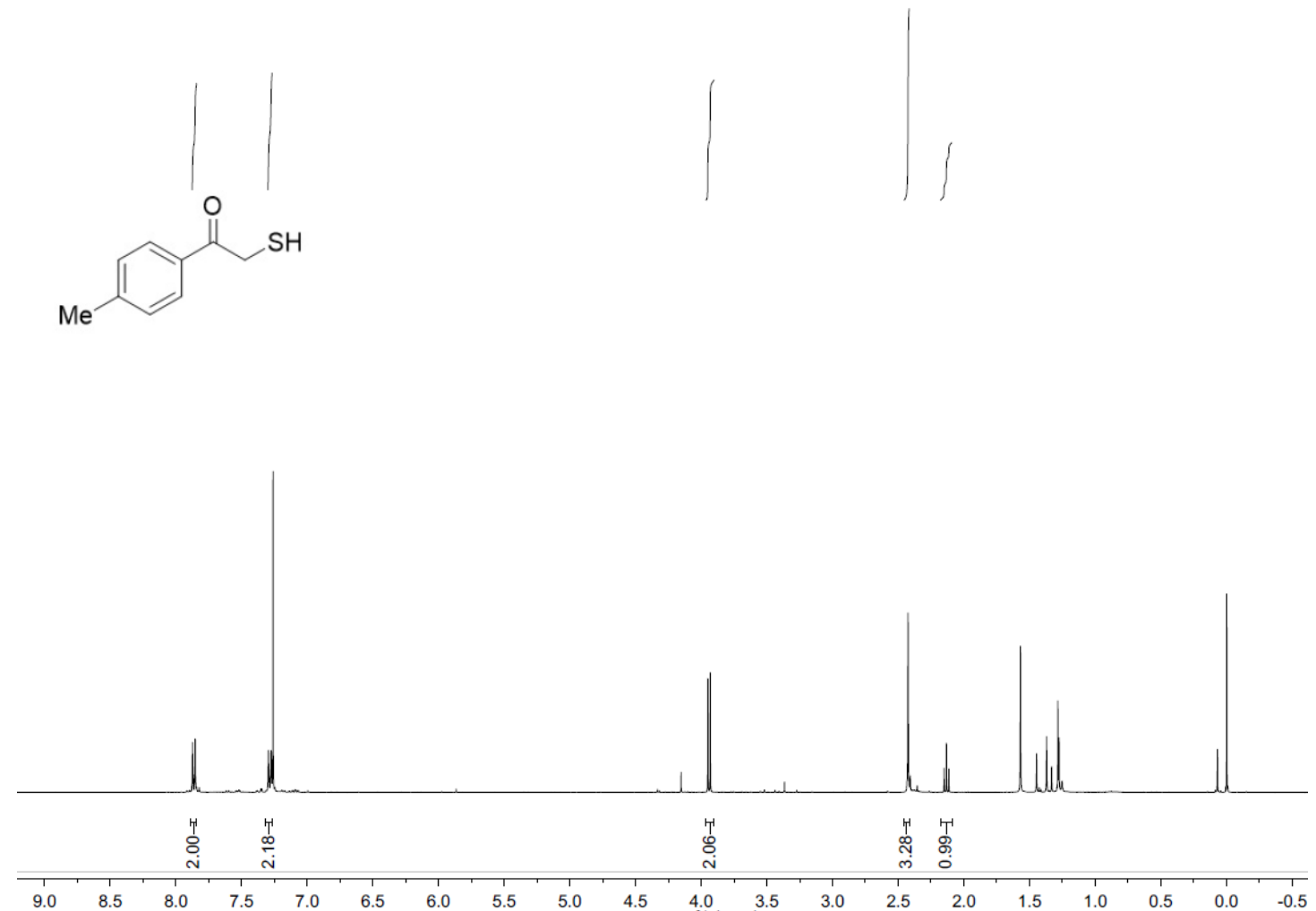

1E:

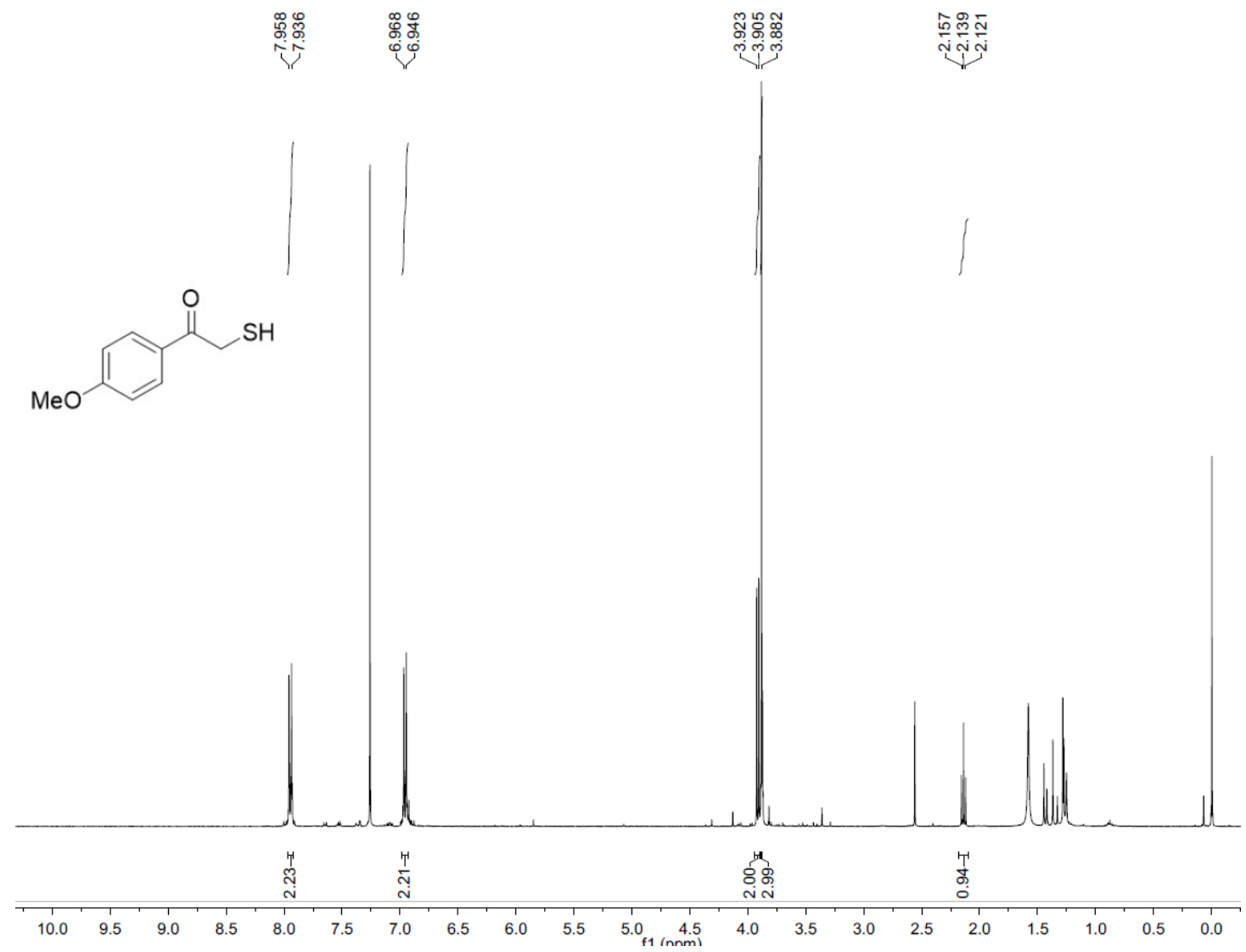


3a:

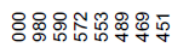

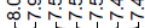

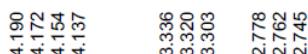

茫

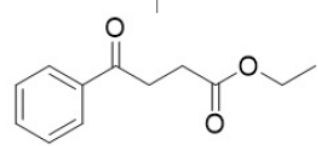

${ }^{1} \mathrm{H} \mathrm{NMR} \mathrm{CDCl}{ }_{3}, 400 \mathrm{MHz}$

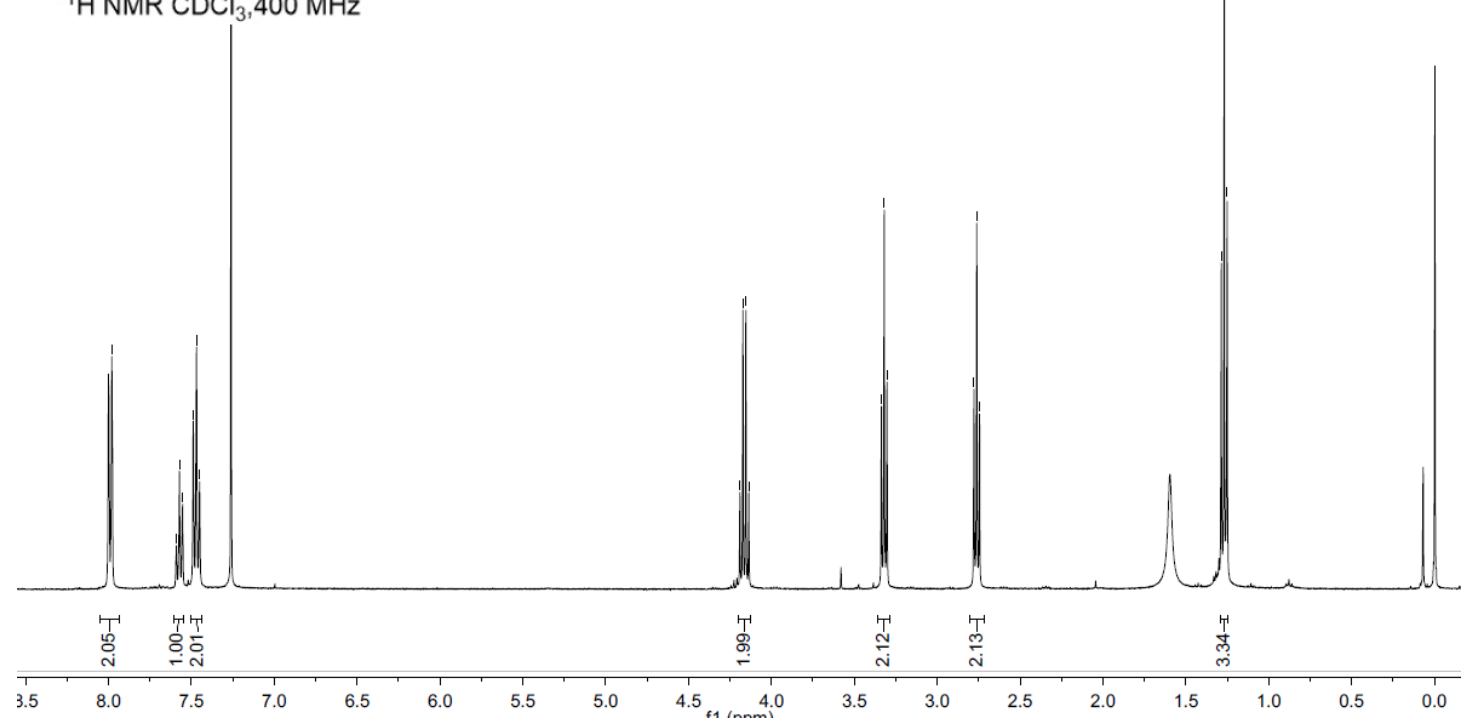

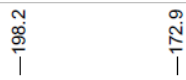

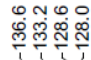

苑管

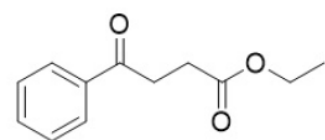

${ }^{13} \mathrm{C}$ NMR $101 \mathrm{MHz}, \mathrm{CDCl}_{3}$

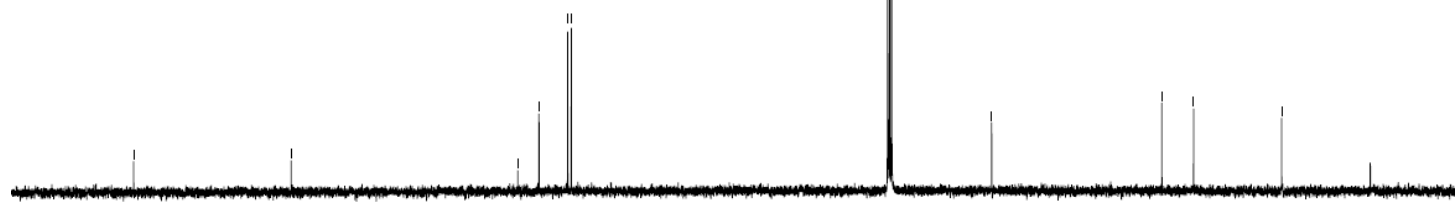

$\begin{array}{llllllllllllllllllllllllllllllllll}210 & 200 & 190 & 180 & 170 & 160 & 150 & 140 & 130 & 120 & 110 & \begin{array}{c}100 \\ \mathrm{f}(\mathrm{ppm})\end{array} & 90 & 80 & 70 & 60 & 50 & 40 & 30 & 20 & 10 & 0 & -10\end{array}$ 
3b:

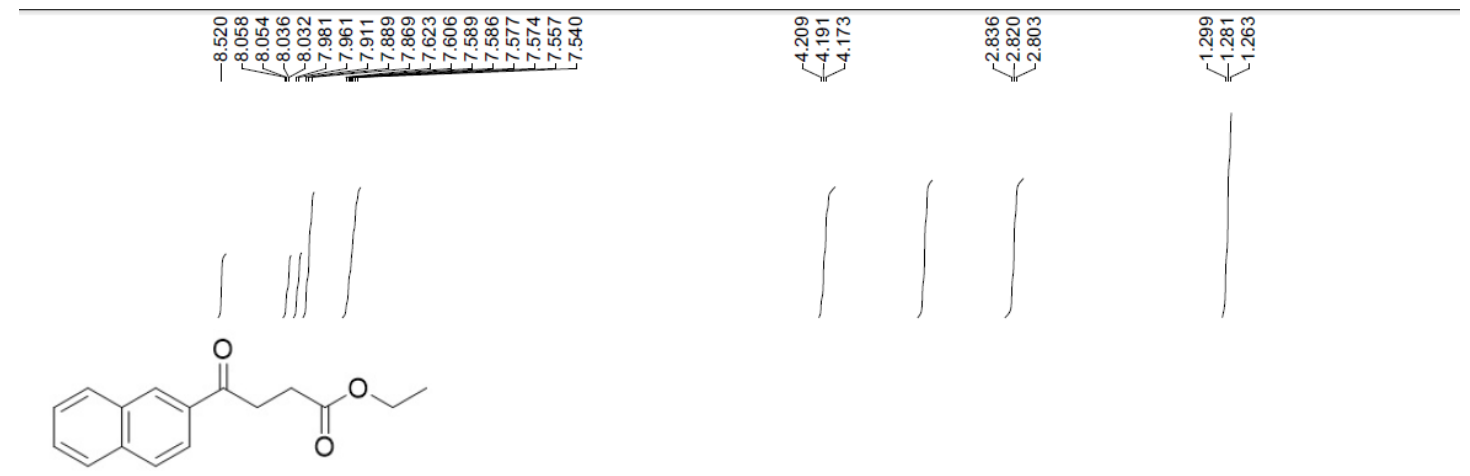

${ }^{1} \mathrm{H}$ NMR $\mathrm{CDCl}_{3} 400 \mathrm{MHz}$
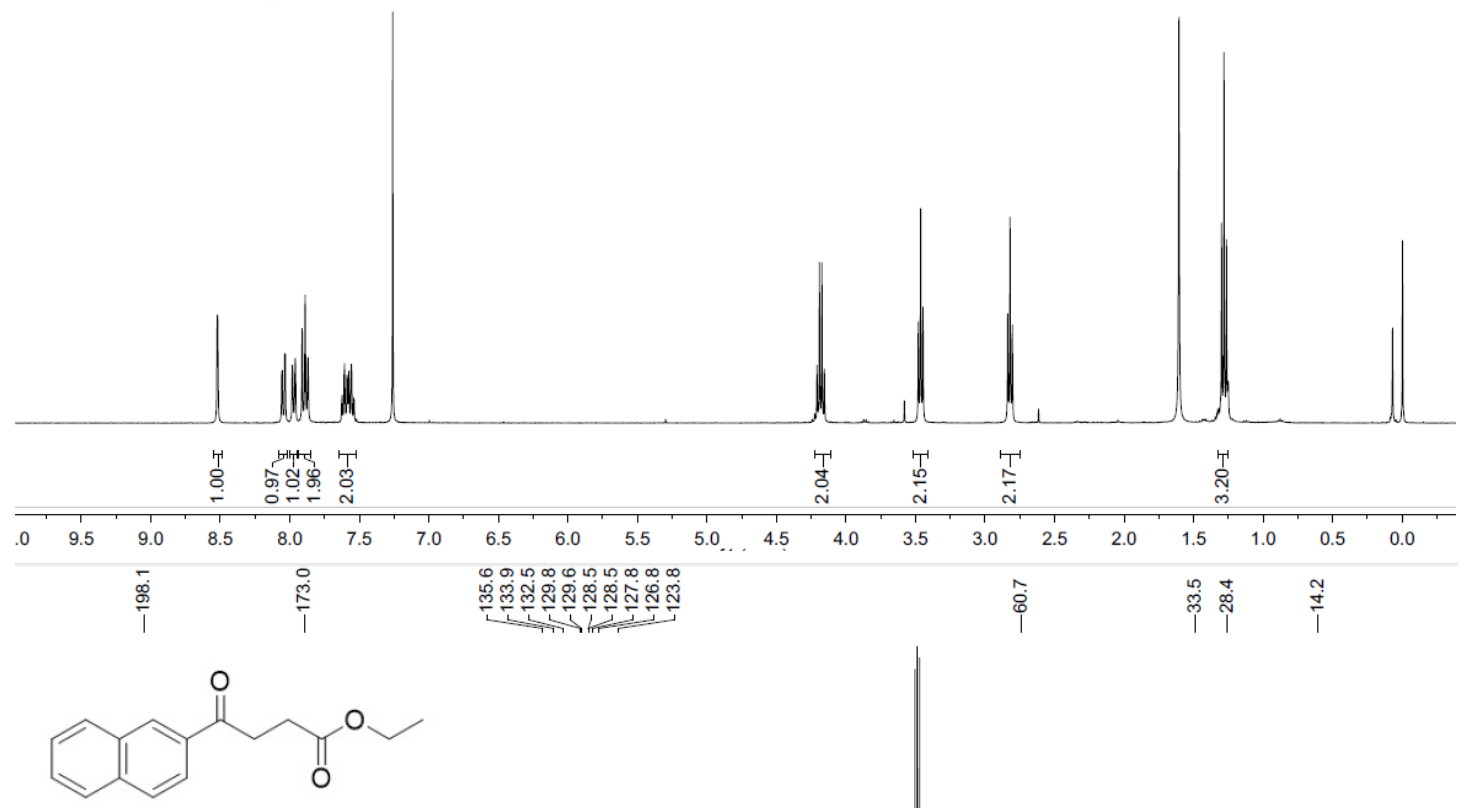

${ }^{13} \mathrm{C} \mathrm{NMR} \mathrm{CDCl}_{3}, 101 \mathrm{MHz}$

$\begin{array}{llllllllllllllllllllllllllllllll}1 & 1 & 200 & 190 & 180 & 170 & 160 & 150 & 140 & 130 & 120 & 110 & 100 & 90 & 80 & 70 & 60 & 50 & 40 & 30 & 20 & 10 & 0\end{array}$ 
3c:

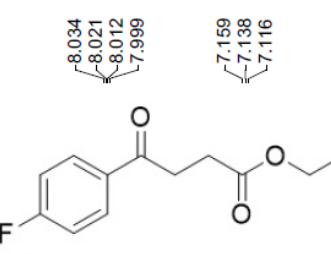

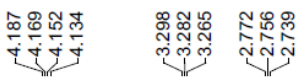

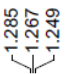

${ }^{1} \mathrm{H} \mathrm{NMR} \mathrm{CDCl}{ }_{3}, 400 \mathrm{MHz}$

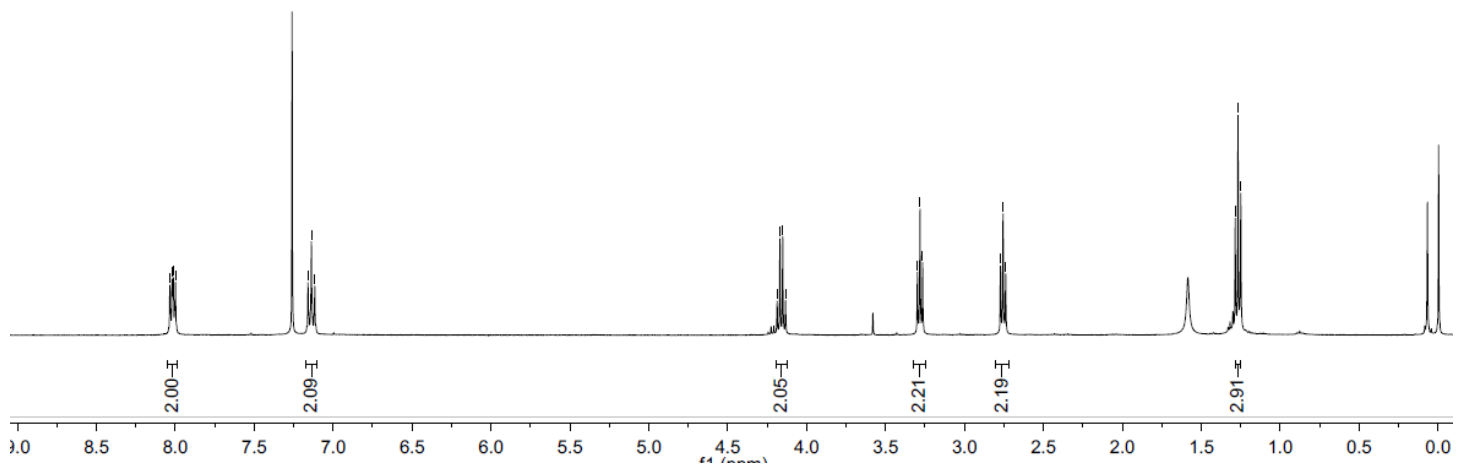

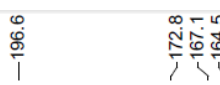

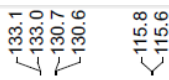

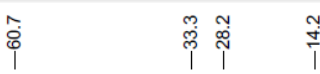

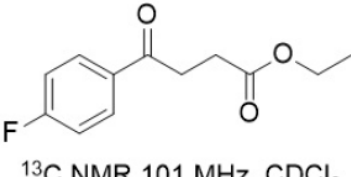

${ }^{13} \mathrm{C}$ NMR $101 \mathrm{MHz}, \mathrm{CDCl}_{3}$

$\begin{array}{llllllllllllllllllllllllllllllllll}210 & 200 & 190 & 180 & 170 & 160 & 150 & 140 & 130 & 120 & 110 & 100 & 90 & 80 & 70 & 60 & 50 & 40 & 30 & 20 & 10 & 0\end{array}$


3d:

$$
\text { 荥年 }
$$

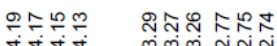

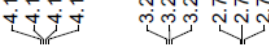

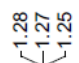<smiles>CCOC(=O)CCC(=O)c1ccc(Cl)cc1</smiles>

${ }^{1} \mathrm{H} \mathrm{NMR} \mathrm{CDCl}{ }_{3}, 400 \mathrm{MHz}$

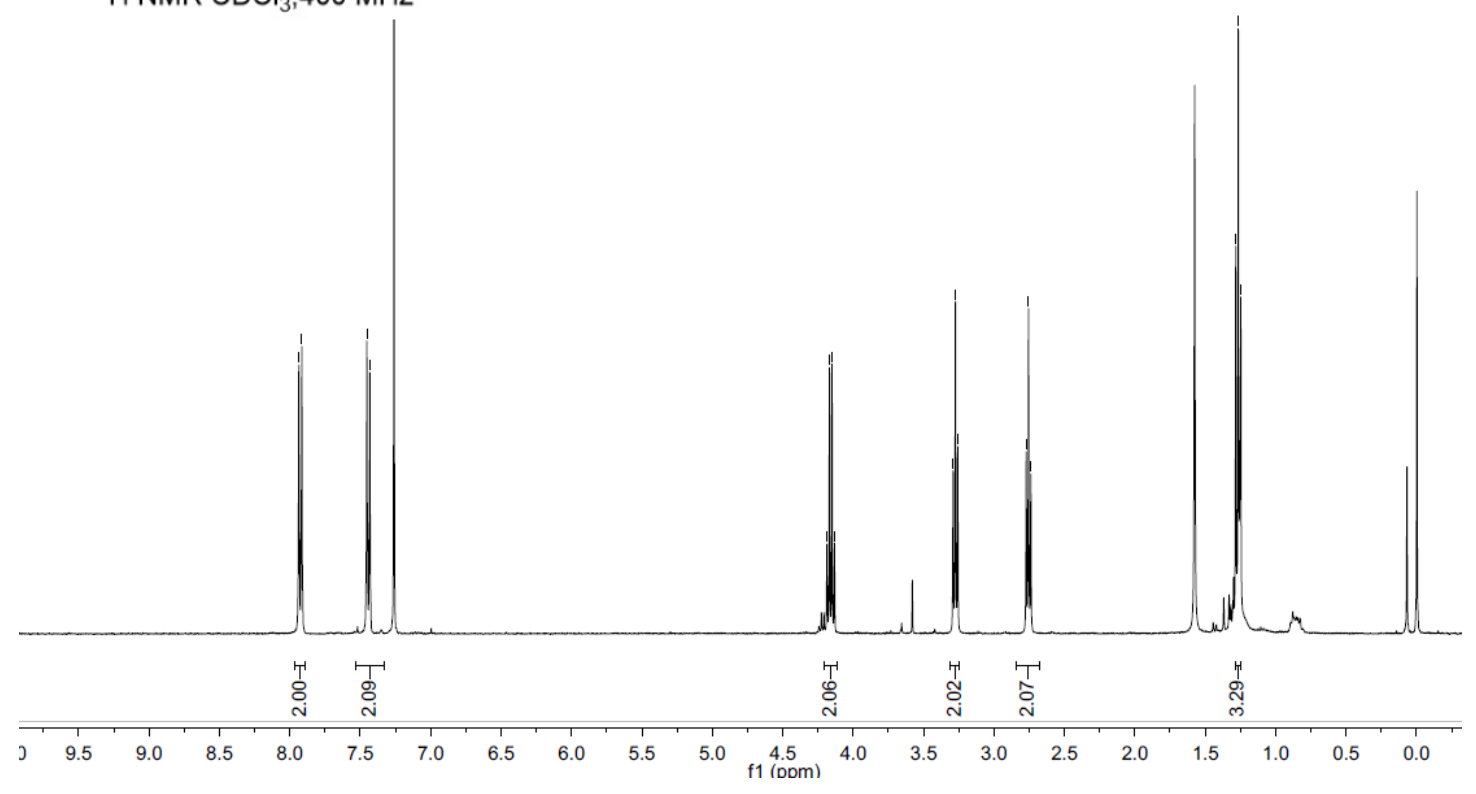

$\stackrel{\substack{i \\ i}}{\stackrel{\infty}{i}}$

กำ

商 $\quad 0$

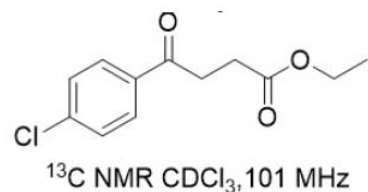

${ }^{13} \mathrm{C} \mathrm{NMR} \mathrm{CDCl}_{3}, 101 \mathrm{MHz}$

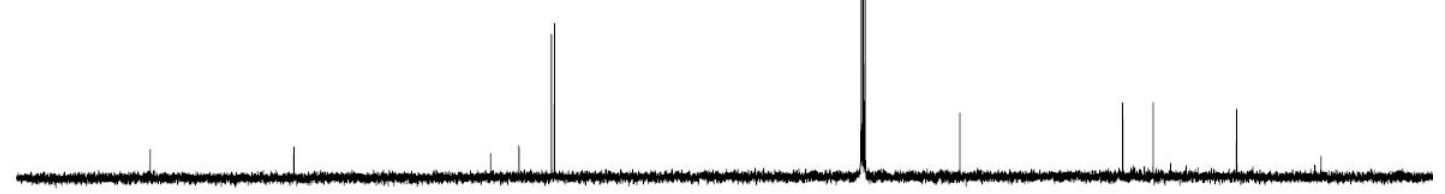

$\begin{array}{lllllllllllllllllllllllllllllll}210 & 200 & 190 & 180 & 170 & 160 & 150 & 140 & 130 & 120 & 110 & 100 & 10 & 10 & 70 & 60 & 50 & 40 & 30 & 20 & 10 & 0 & -10\end{array}$ 
3e:

-.

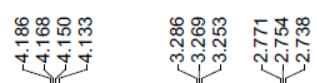

荧
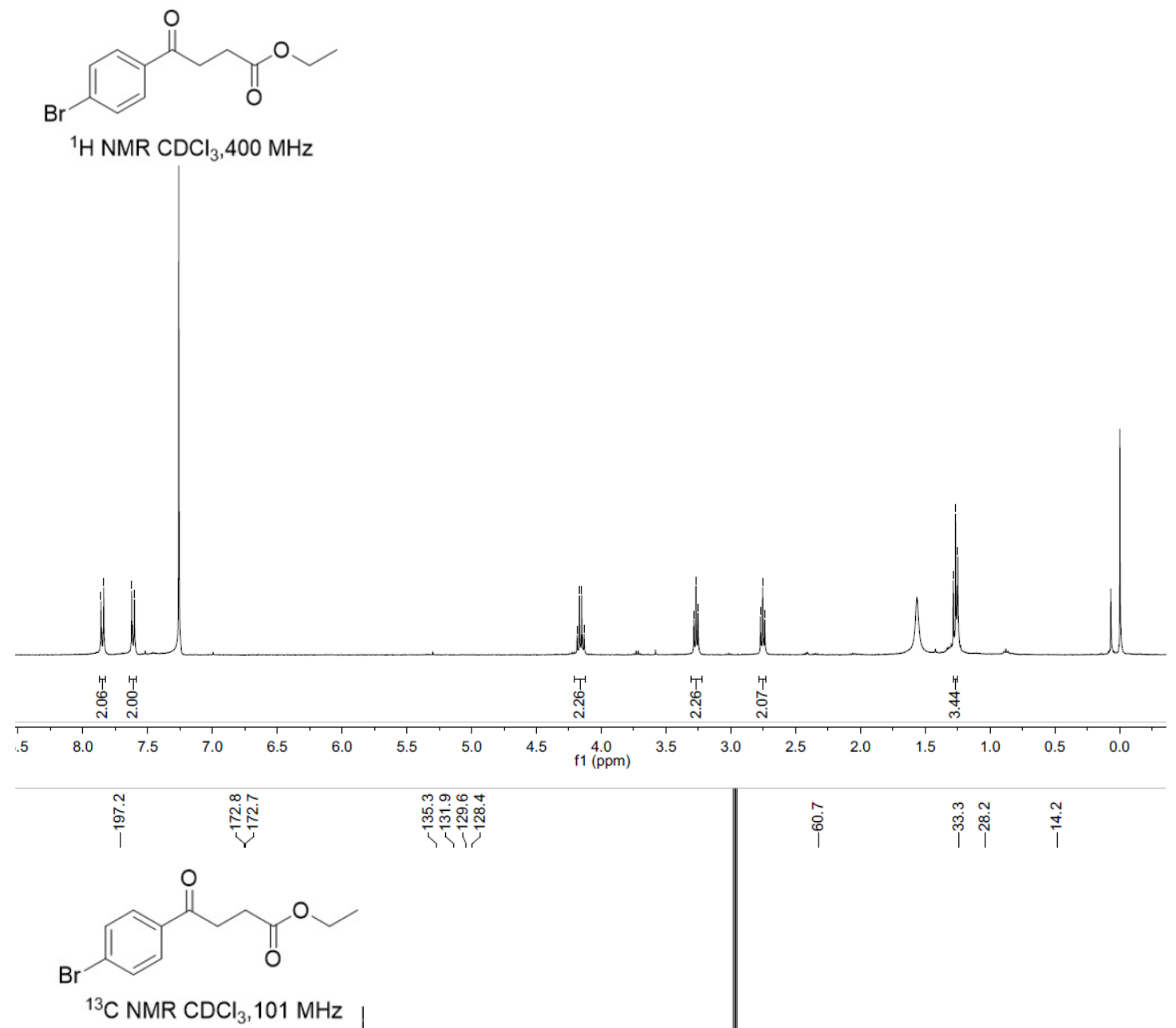

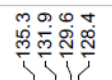

î̀

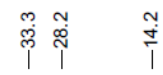

${ }^{13} \mathrm{C} \mathrm{NMR} \mathrm{CDCl} 3,101 \mathrm{MHz}$

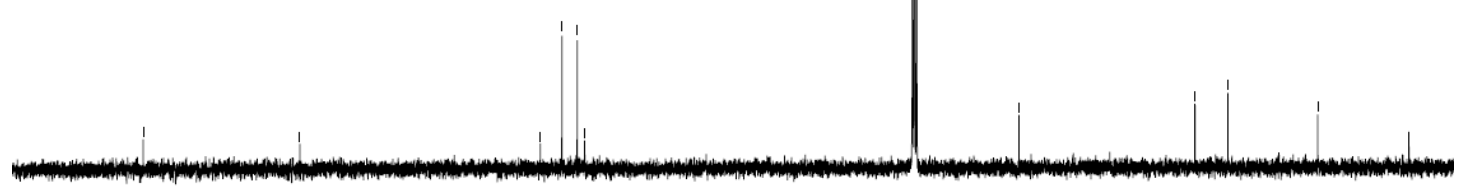

$\begin{array}{llllllllllllllllllllllllllllllll}210 & 200 & 190 & 180 & 170 & 160 & 150 & 140 & 130 & 120 & 110 & 100 & 90 & 80 & 70 & 60 & 50 & 40 & 30 & 20 & 10 & 0\end{array}$ 
3f:

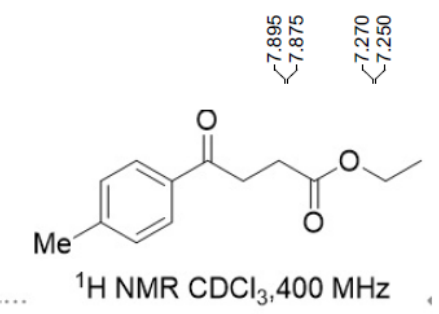

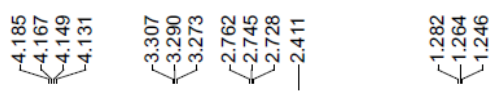

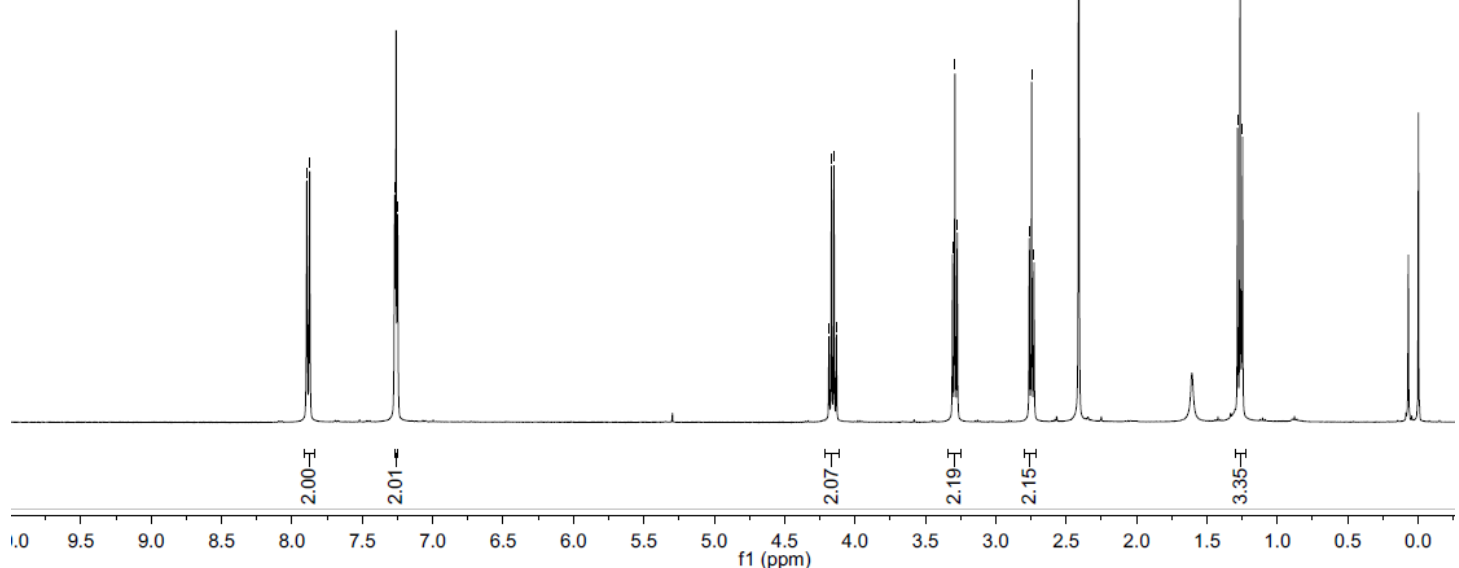

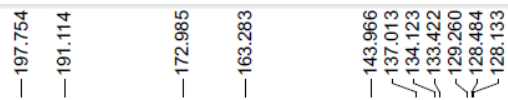

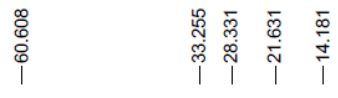

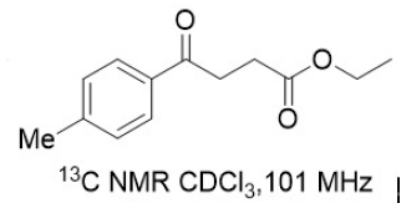

${ }^{13} \mathrm{C} \mathrm{NMR} \mathrm{CDCl}_{3}, 101 \mathrm{MHz}$

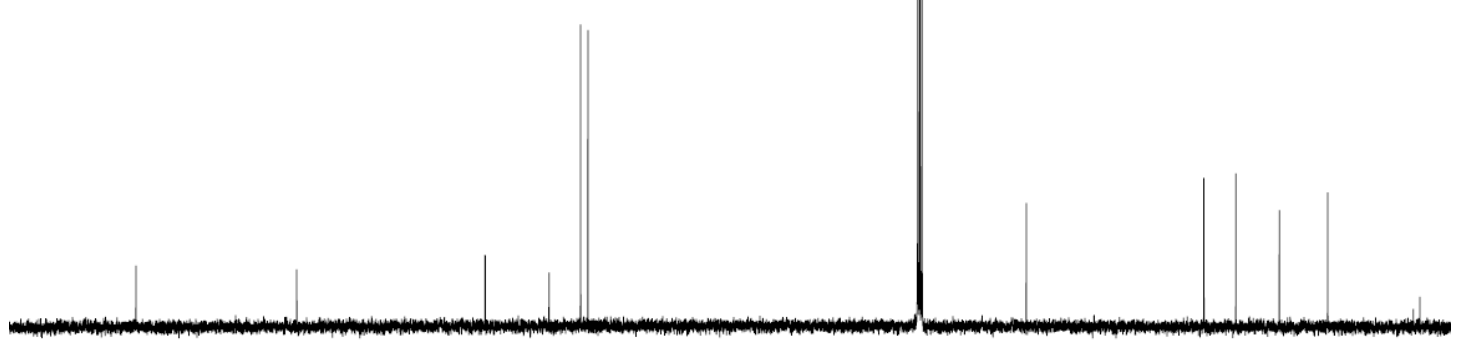

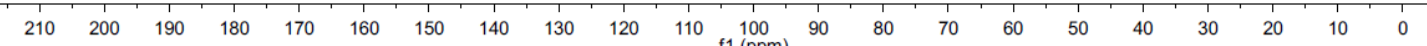


3g:

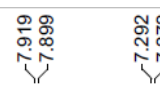

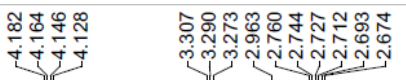<smiles>CCOC(=O)CCC(=O)c1ccc(CC)cc1</smiles>

${ }^{1} \mathrm{H} \mathrm{NMR} \mathrm{CDCl}{ }_{3}, 400 \mathrm{MHz}$

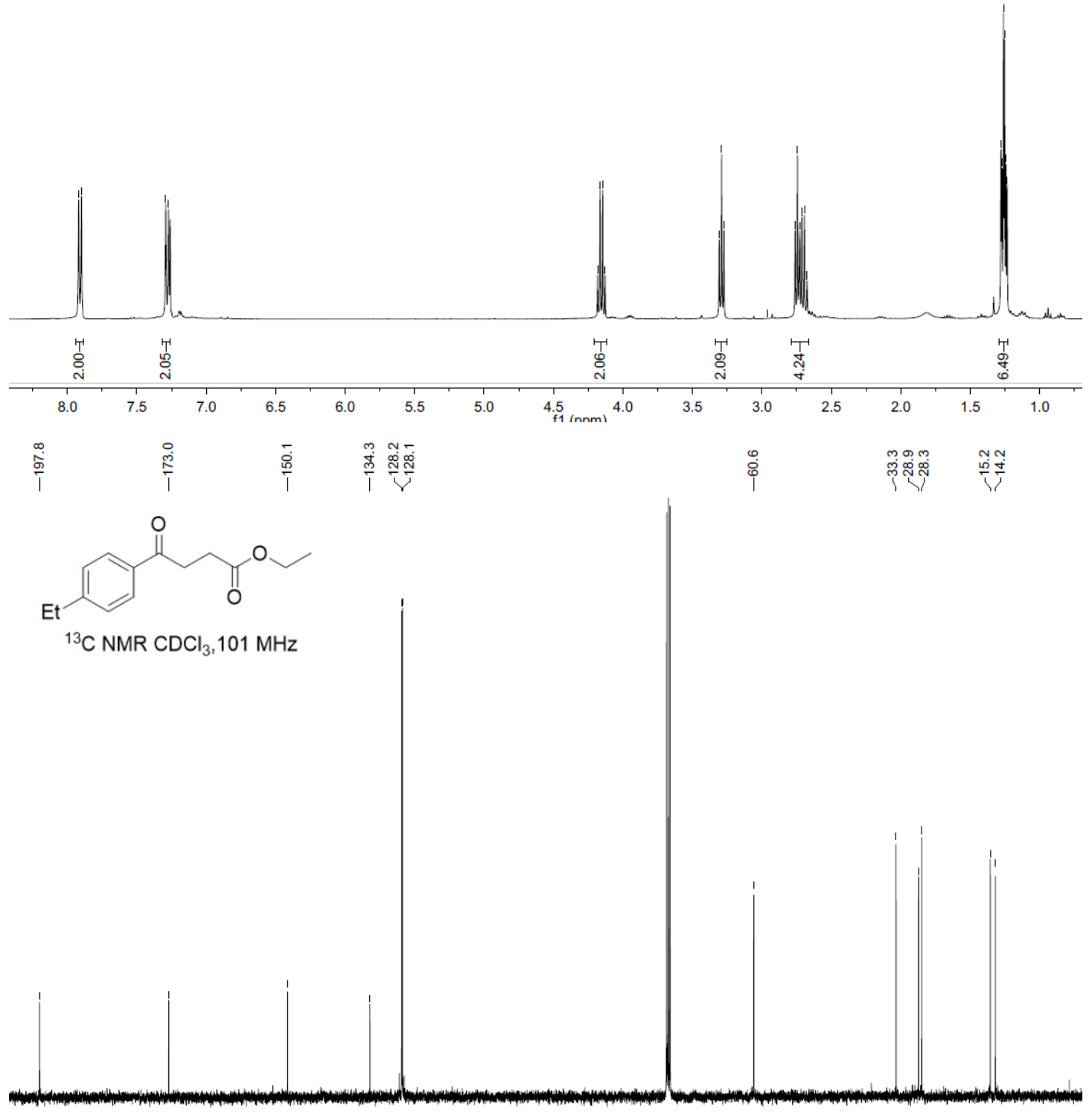

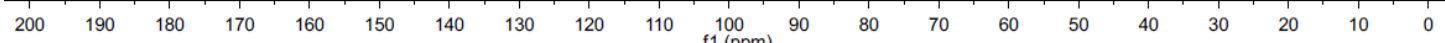


3h:

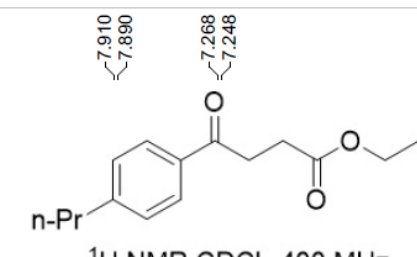

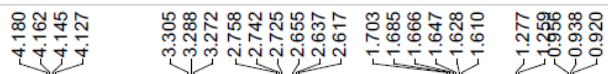

${ }^{1} \mathrm{H} \mathrm{NMR} \mathrm{CDCl}_{3}, 400 \mathrm{MHz}$

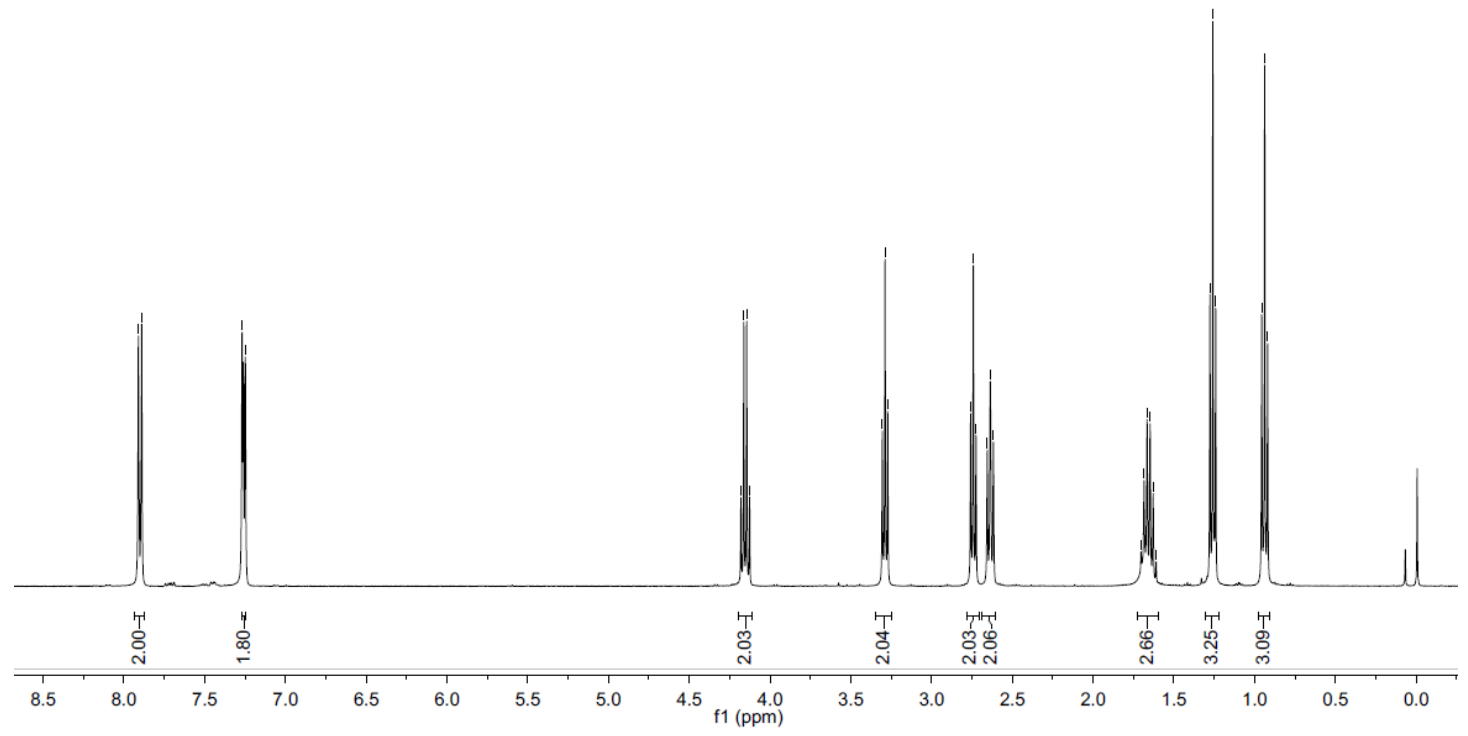<smiles></smiles>

${ }^{13} \mathrm{C} \mathrm{NMR} \mathrm{CDCl}_{3}, 101 \mathrm{MHz}$

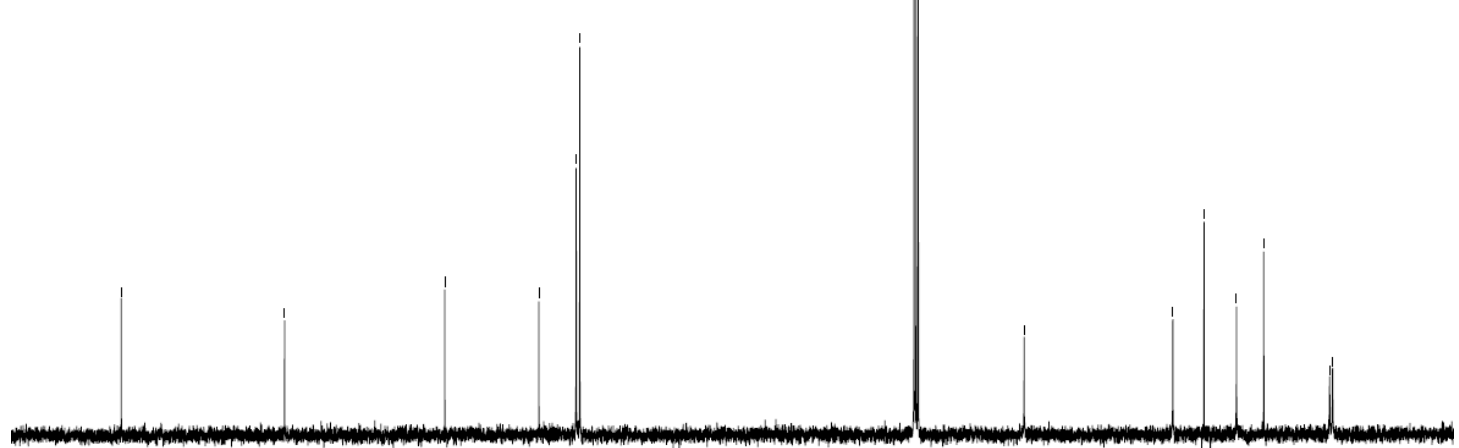

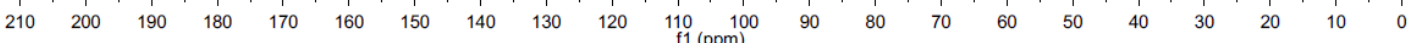


3i:

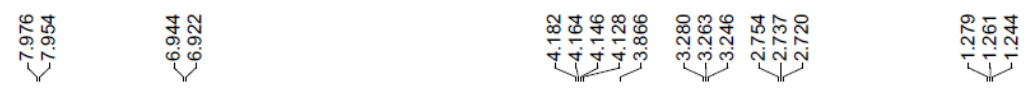<smiles>CCOC(=O)CCC(=O)c1ccc(OC)cc1</smiles>

${ }^{1} \mathrm{H} \mathrm{NMR} \mathrm{CDCl}{ }_{3}, 400 \mathrm{MHz}$

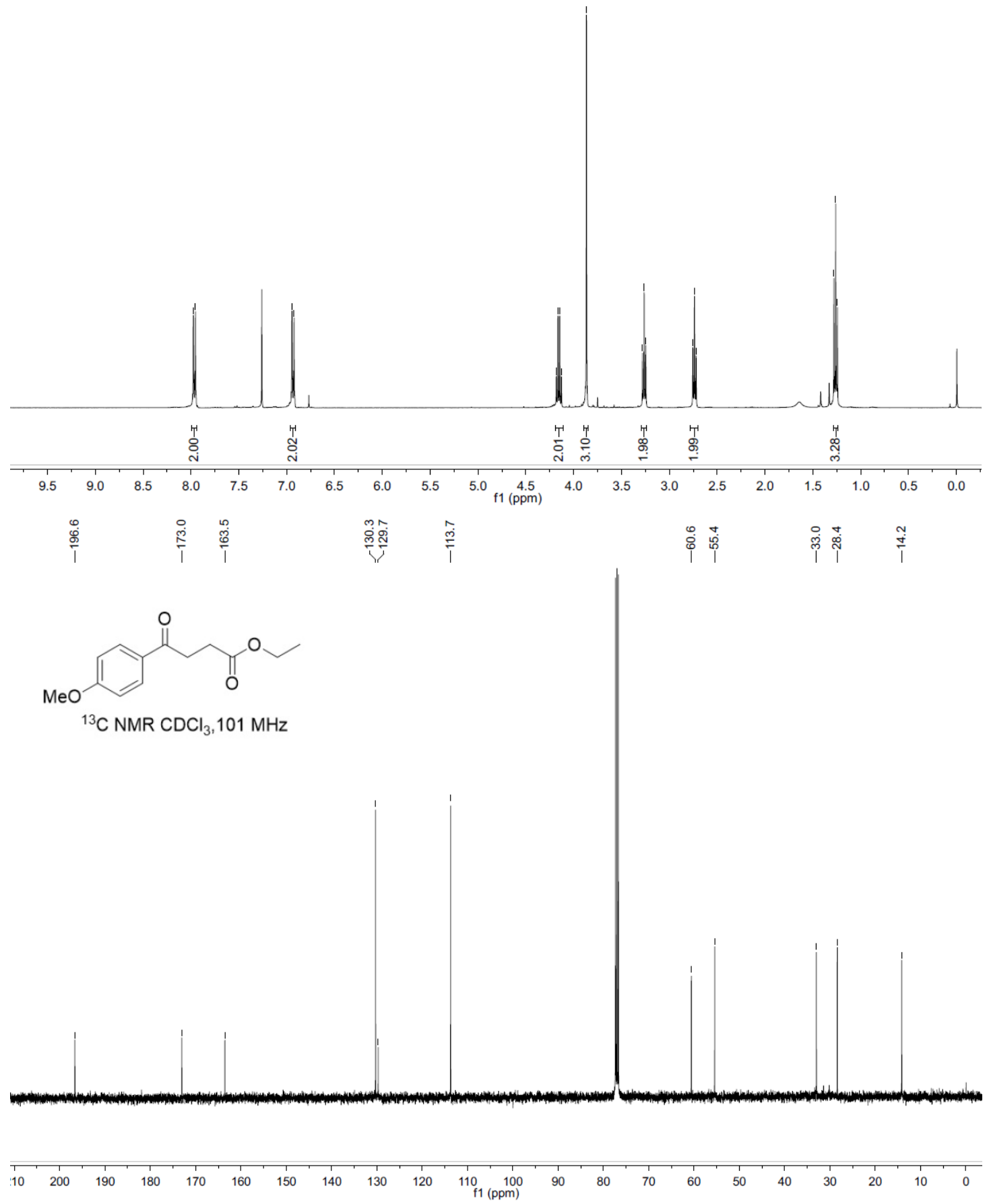


3j:

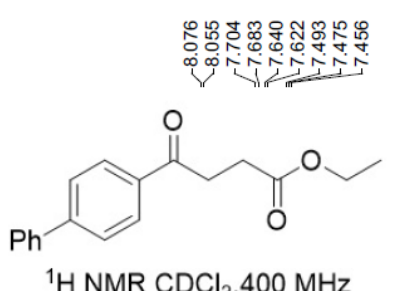

${ }^{1} \mathrm{H} \mathrm{NMR} \mathrm{CDCl}_{3}, 400 \mathrm{MHz}$

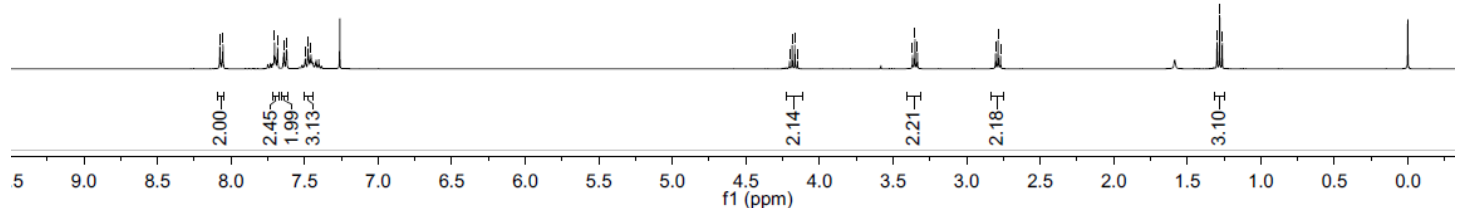

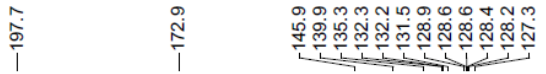

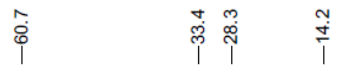<smiles>CCOC(=O)CCC(=O)c1ccc(-c2ccccc2)cc1</smiles>

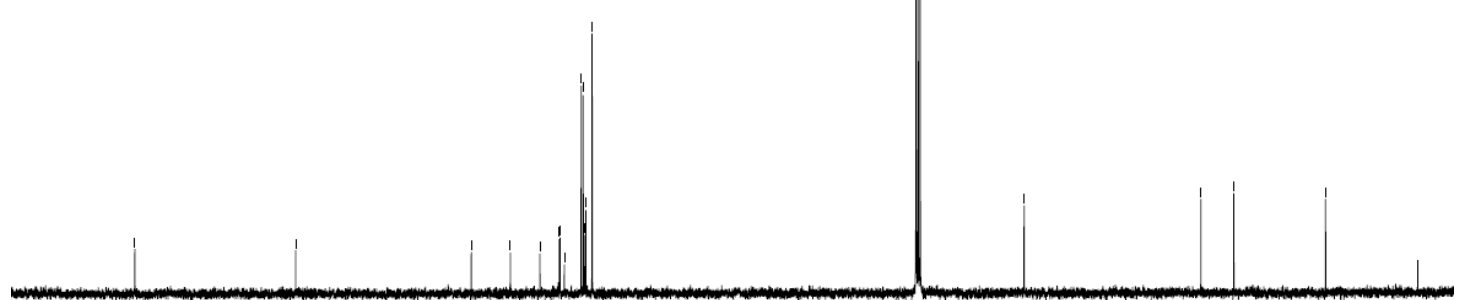

$\begin{array}{llllllllllllllllllllllllllllll}\mid & 1 \\ 210 & 200 & 190 & 180 & 170 & 160 & 150 & 140 & 130 & 120 & 110 & 100 & 90 & 80 & 70 & 60 & 50 & 40 & 30 & 20 & 10 & 0\end{array}$ 
3k:

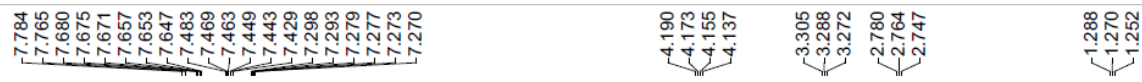

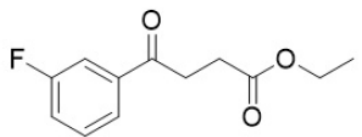

${ }^{1} \mathrm{H} \mathrm{NMR} \mathrm{CDCl}_{3}, 400 \mathrm{MHz}$

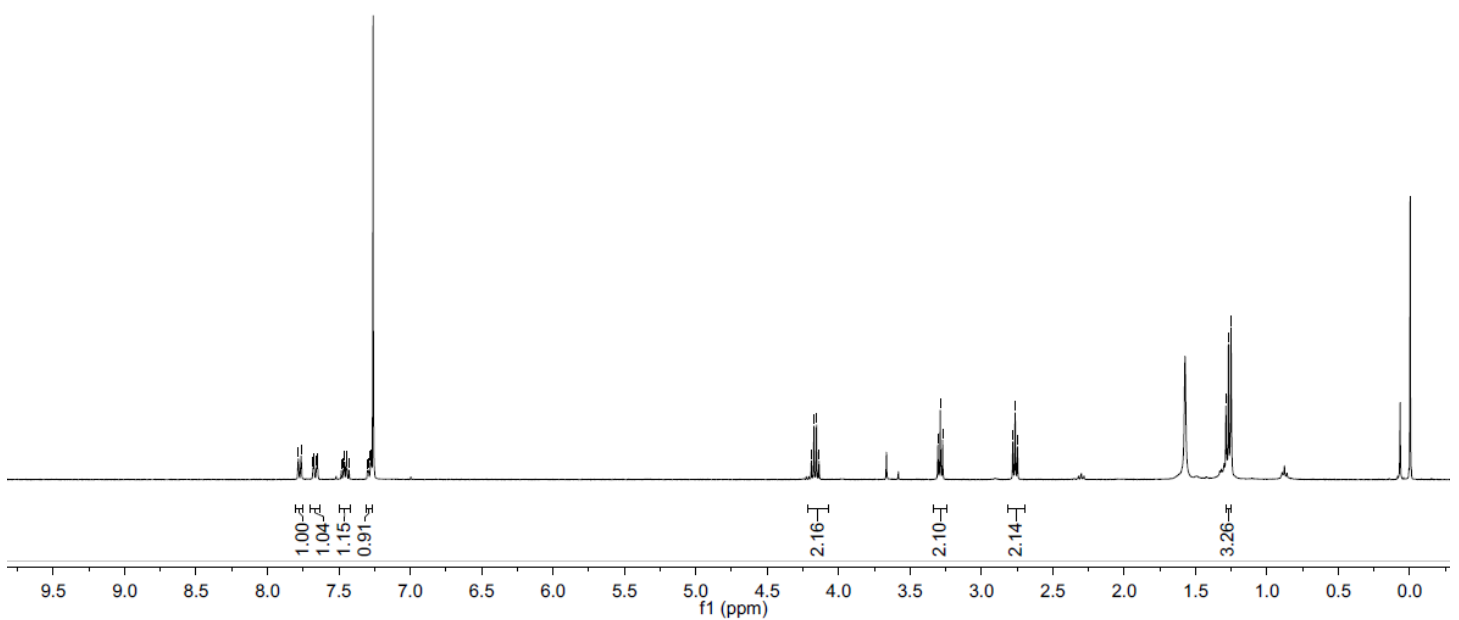

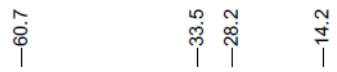

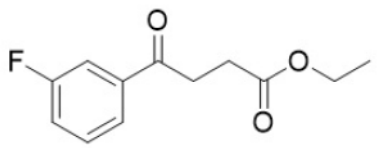

${ }^{13} \mathrm{C} \mathrm{NMR} \mathrm{CDCl}_{3}, 101 \mathrm{MHz}$

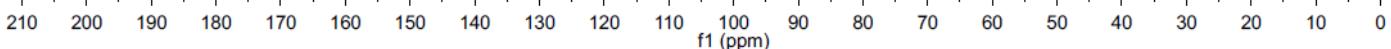


31:

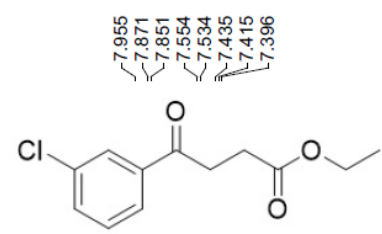

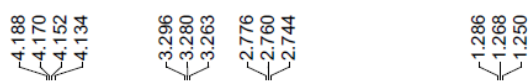

${ }^{1} \mathrm{H} \mathrm{NMR} \mathrm{CDCl}_{3}, 400 \mathrm{MHz}$
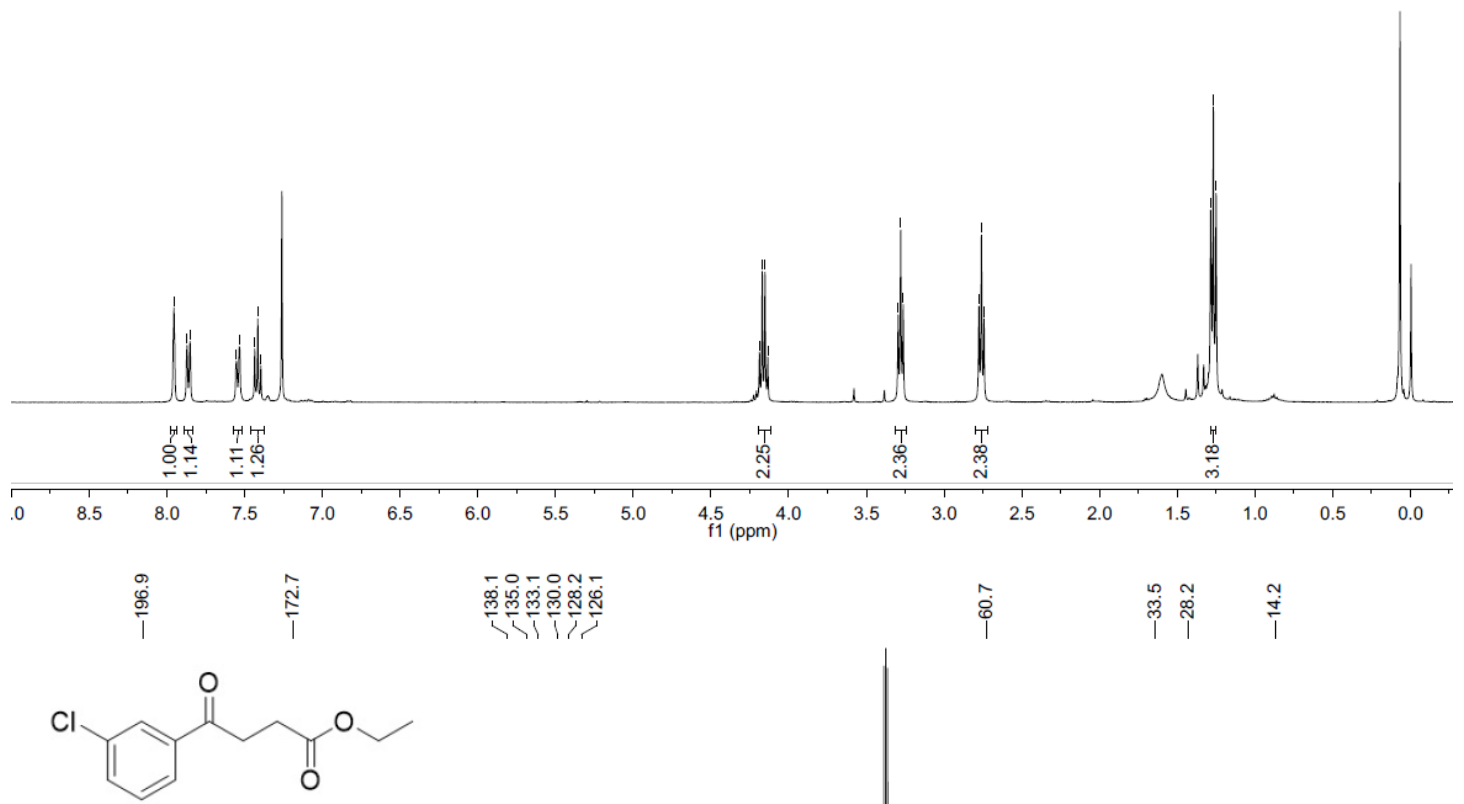

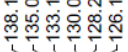

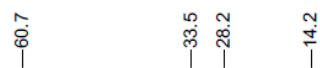

${ }^{13} \mathrm{C} \mathrm{NMR} \mathrm{CDCl}{ }_{3}, 101 \mathrm{MHz}$

$\begin{array}{llllllllllllllllllllllllllll}210 & 200 & 190 & 180 & 170 & 160 & 150 & 140 & 130 & 120 & 110 & 100 & 90 & 80 & 70 & 60 & 50 & 40 & 30 & 20 & 10 & 0 & -10\end{array}$ 
3m:

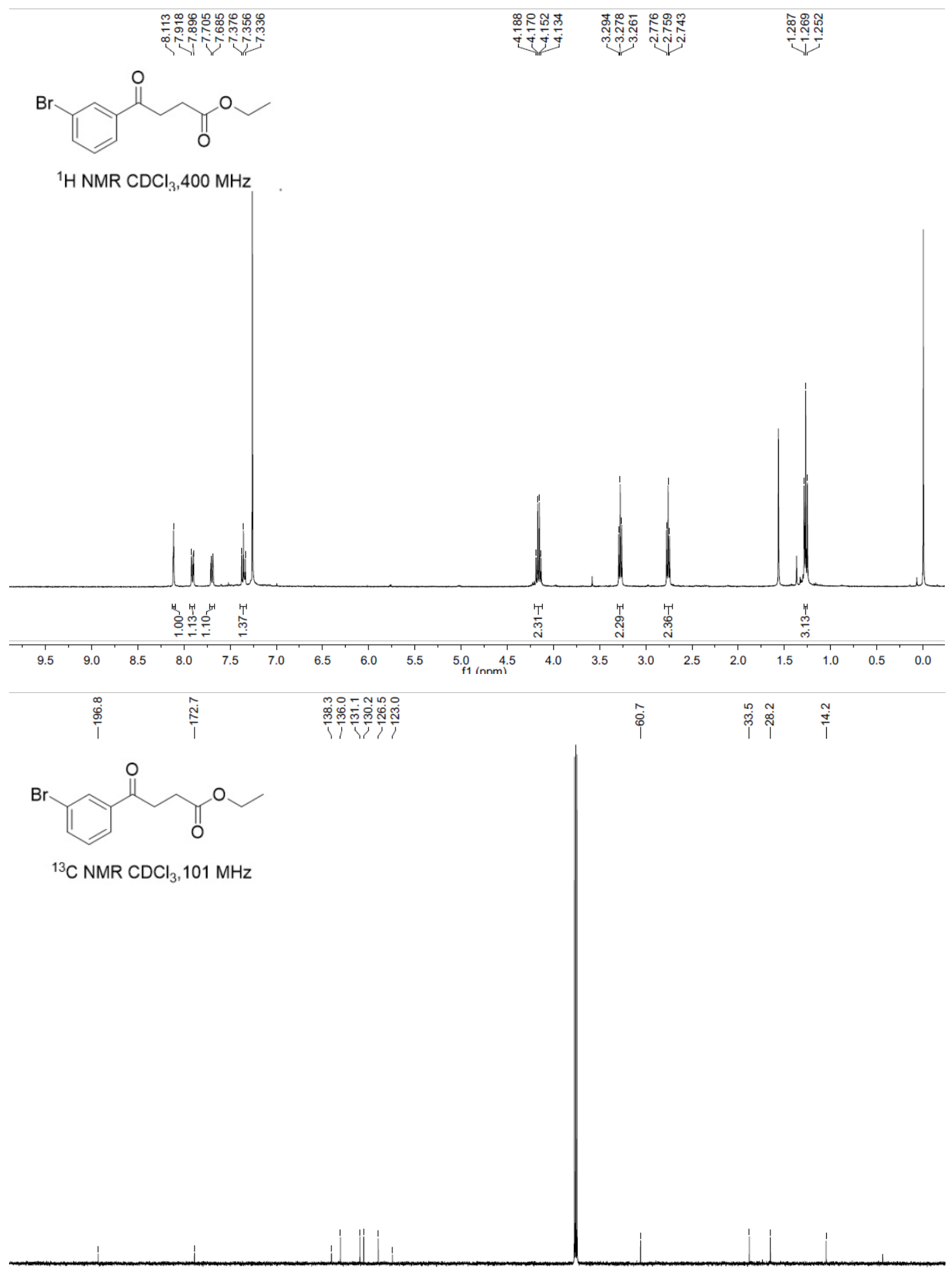

$\begin{array}{lllllllllllllllllllllllllllll}210 & 200 & 190 & 180 & 170 & 160 & 150 & 140 & 130 & 120 & 110 & 100 & 90 & 80 & 70 & 60 & 50 & 40 & 30 & 20 & 10 & 0 & -10\end{array}$ 
3n:
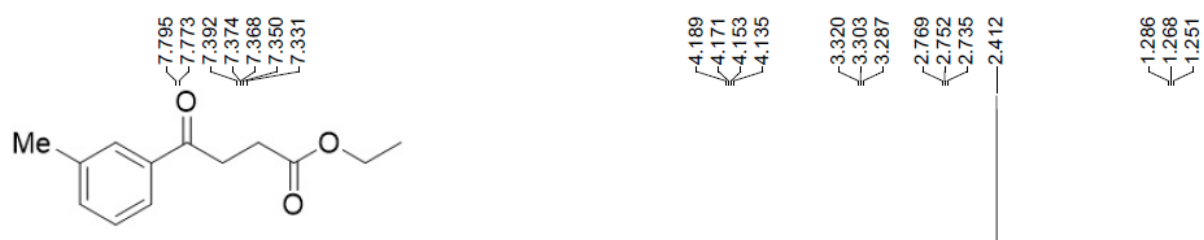

${ }^{1} \mathrm{H} \mathrm{NMR} \mathrm{CDCl}{ }_{3}, 400 \mathrm{MHz}$
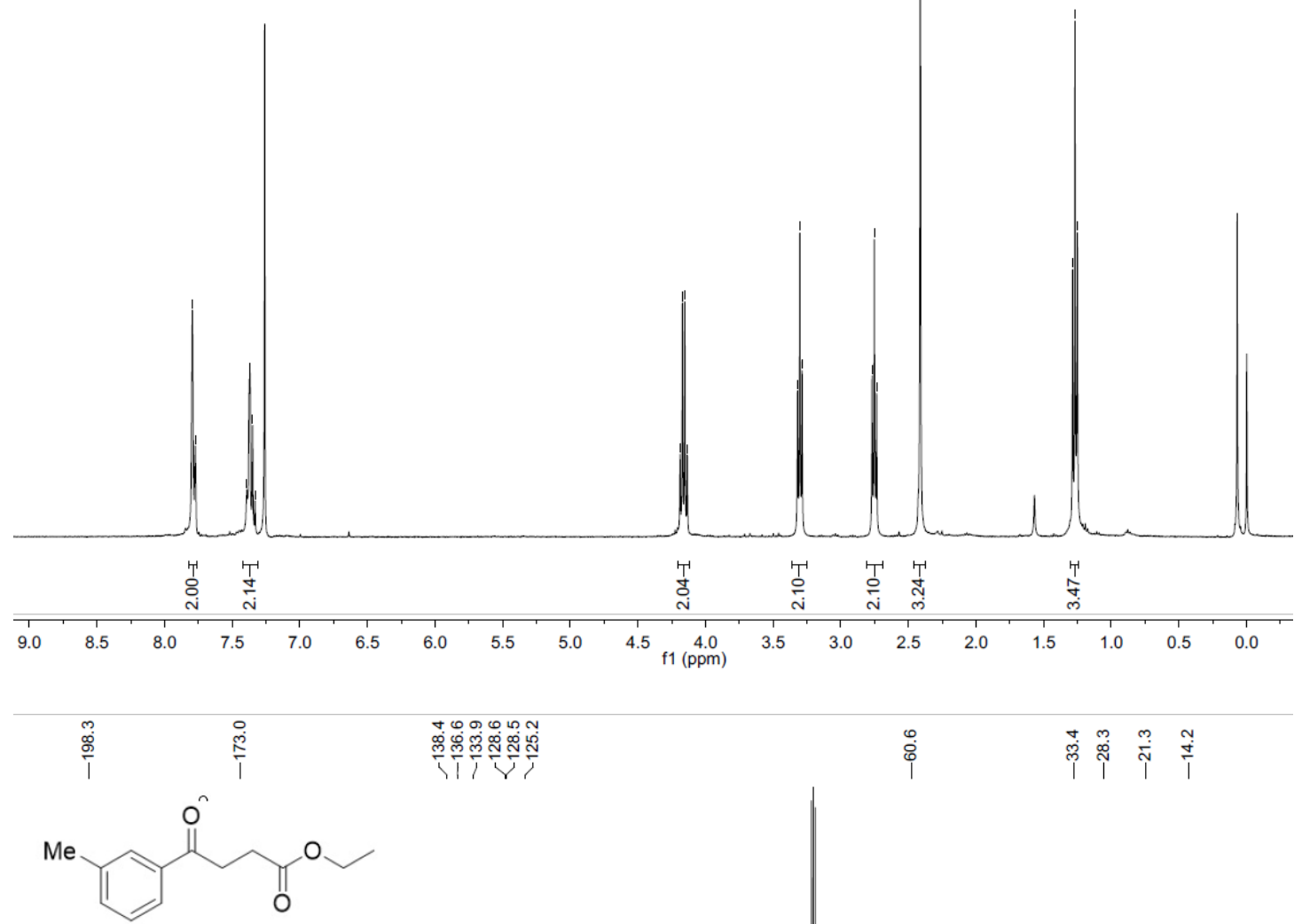

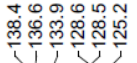

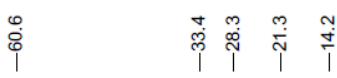

${ }^{13} \mathrm{C} \mathrm{NMR} \mathrm{CDCl}_{3}, 101 \mathrm{MHz}$

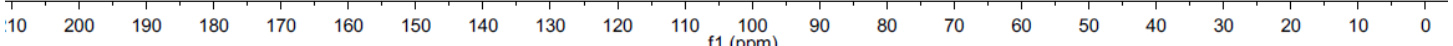


3o:

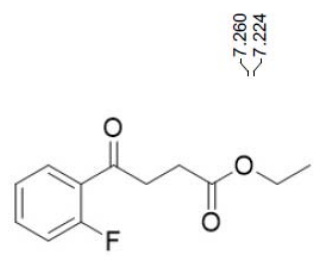

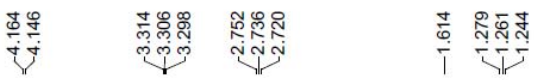

${ }^{1} \mathrm{H} \mathrm{NMR} \mathrm{CDCl}{ }_{3}, 400 \mathrm{MHz}$

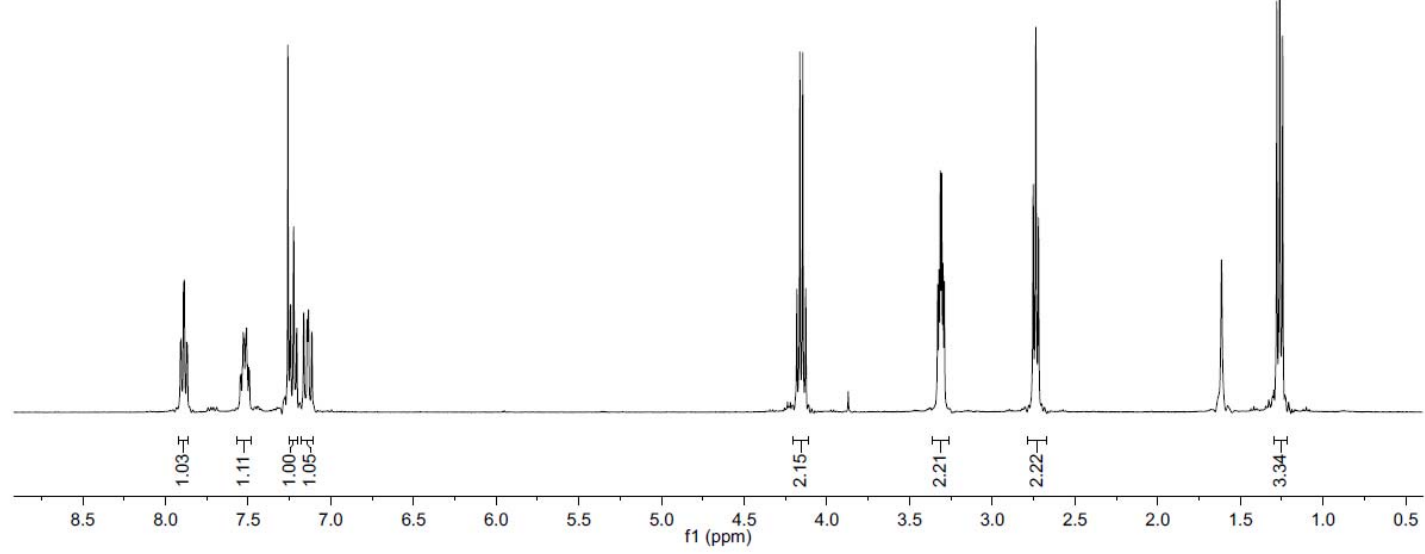

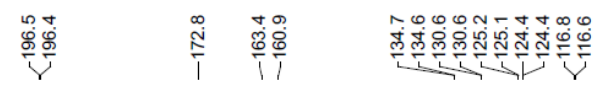

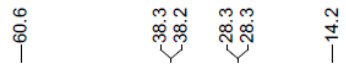

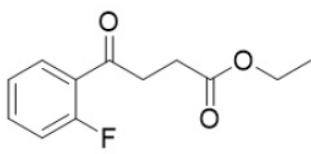

${ }^{13} \mathrm{CNMR} \mathrm{CDCl}_{3}, 101 \mathrm{MHz}$

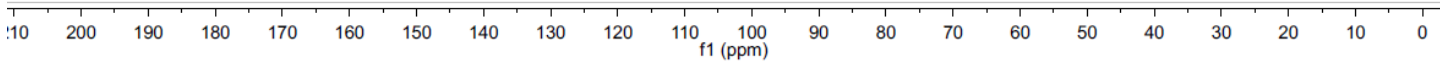


3p:

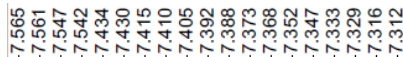

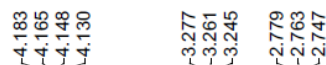

究

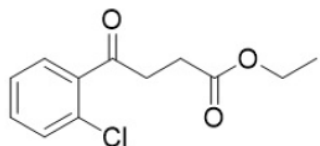

${ }^{1} \mathrm{H} \mathrm{NMR} \mathrm{CDCl} 3,400 \mathrm{MHz}$
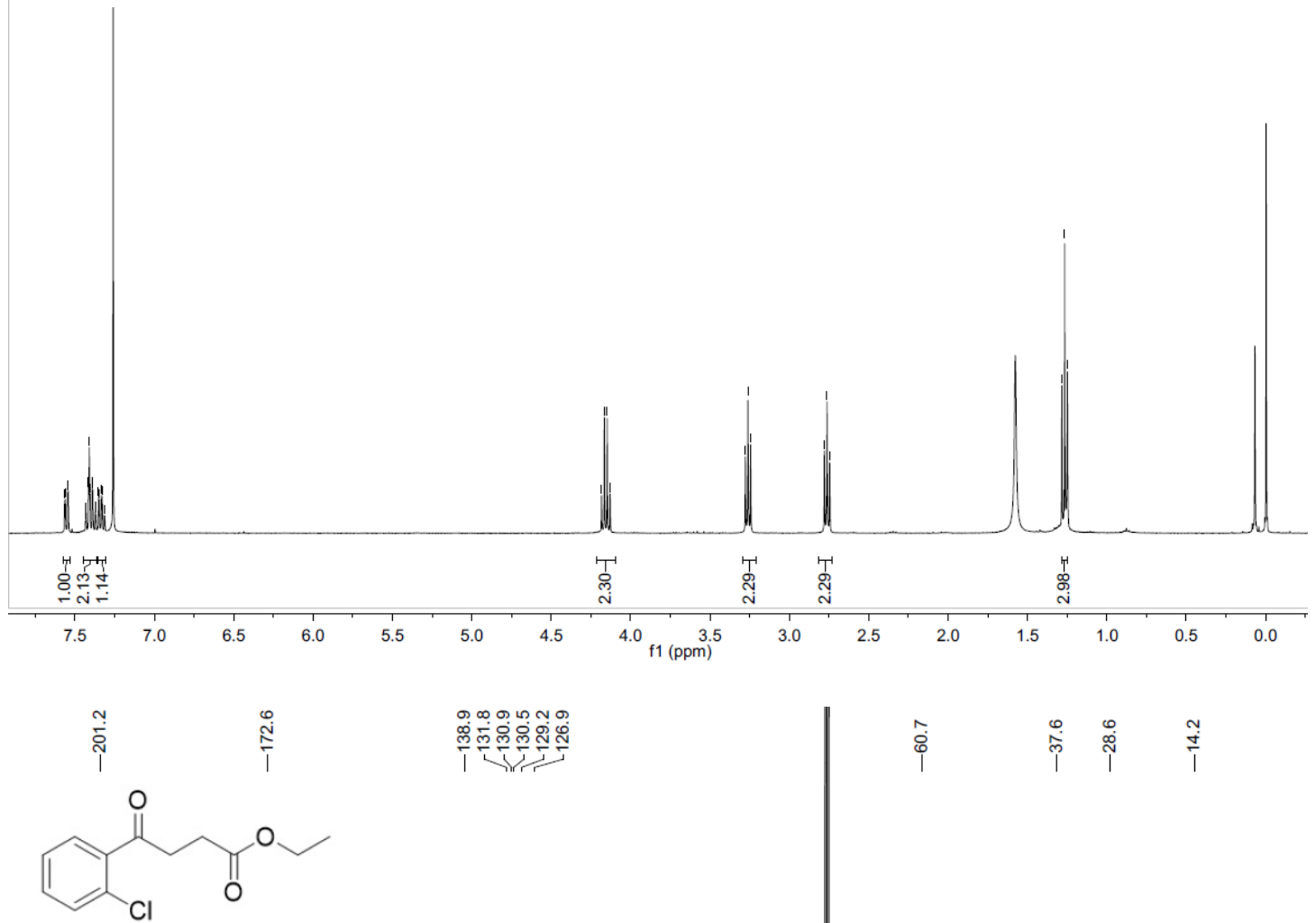

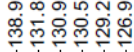

广ิ

${ }^{13} \mathrm{CNMR} \mathrm{CDCl}_{3}, 101 \mathrm{MHz}$

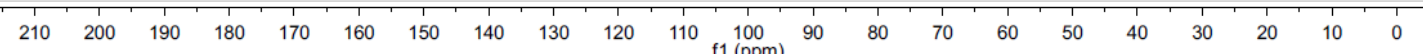


3q:

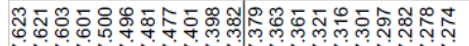

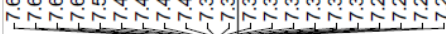

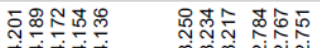

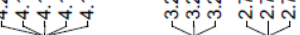

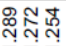

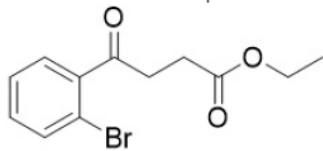

${ }^{1} \mathrm{H} \mathrm{NMR} \mathrm{CDCl}_{3}, 400 \mathrm{MHz}$
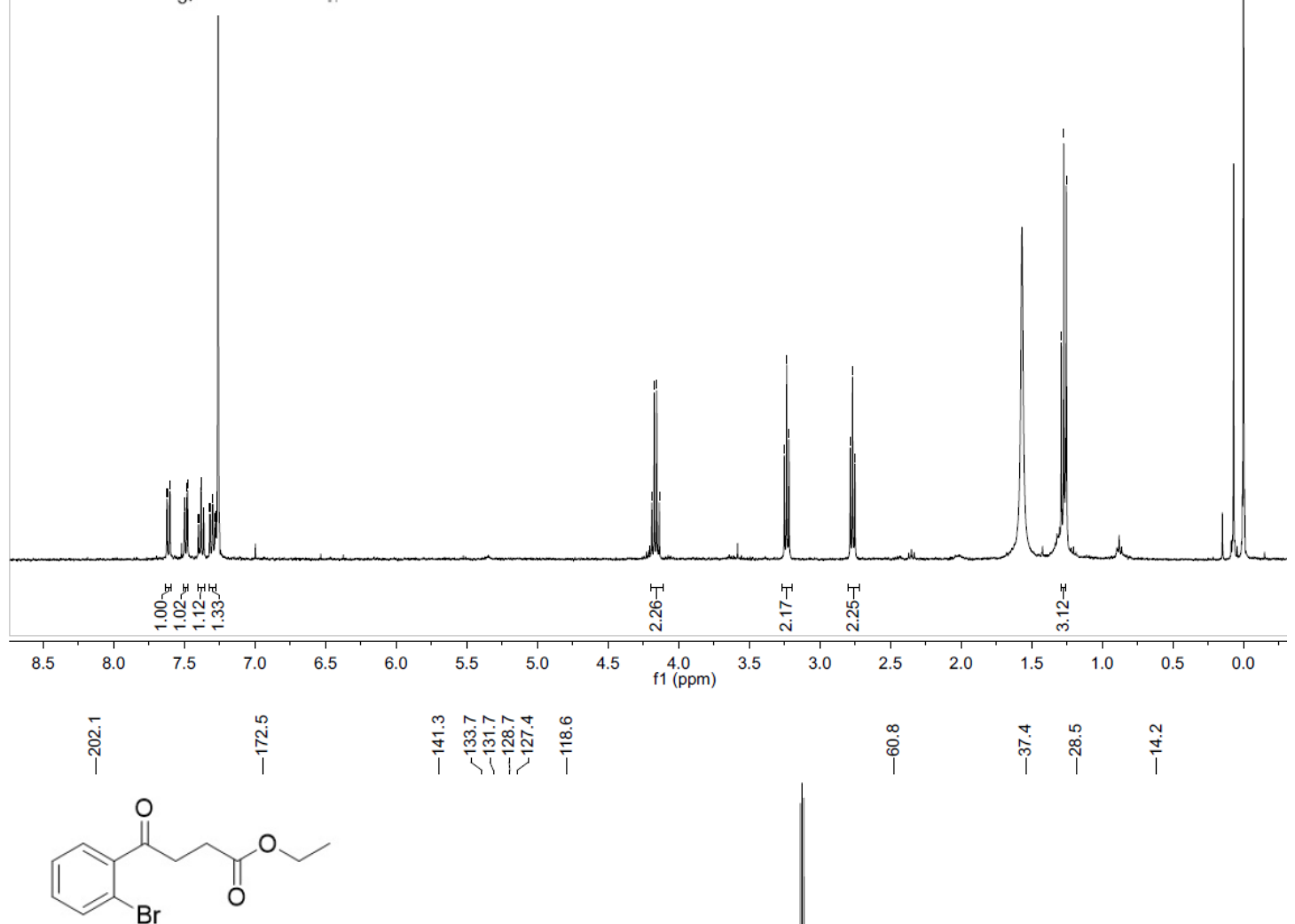

${ }^{13} \mathrm{C} \mathrm{NMR} \mathrm{CDCl}_{3}, 101 \mathrm{MHz}$

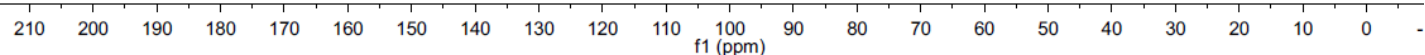


3r:

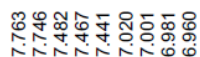

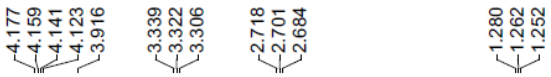

$\overbrace{\mathrm{OMe}}^{\mathrm{O}}$

${ }^{1} \mathrm{H} \mathrm{NMR} \mathrm{CDCl}{ }_{3}, 400 \mathrm{MHz}$

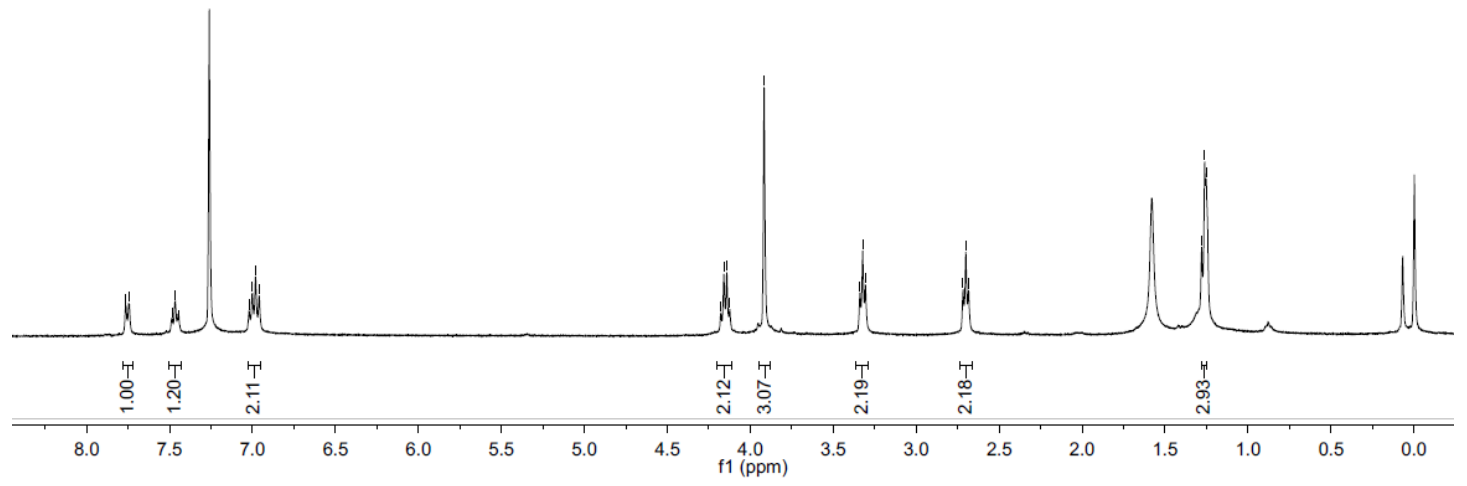

离

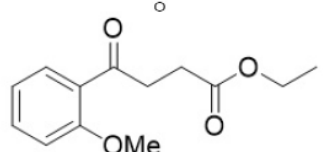

${ }^{13} \mathrm{C} \mathrm{NMR} \mathrm{CDCl} 3,101 \mathrm{MHz}$

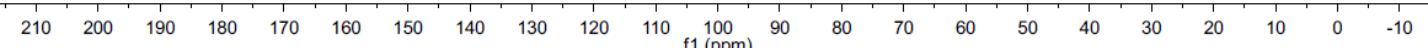


3s:

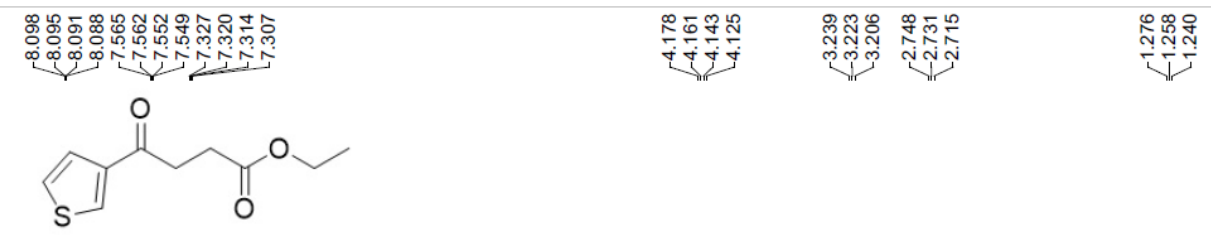

${ }^{1} \mathrm{H} \mathrm{NMR} \mathrm{CDCl}_{3}, 400 \mathrm{MHz}$
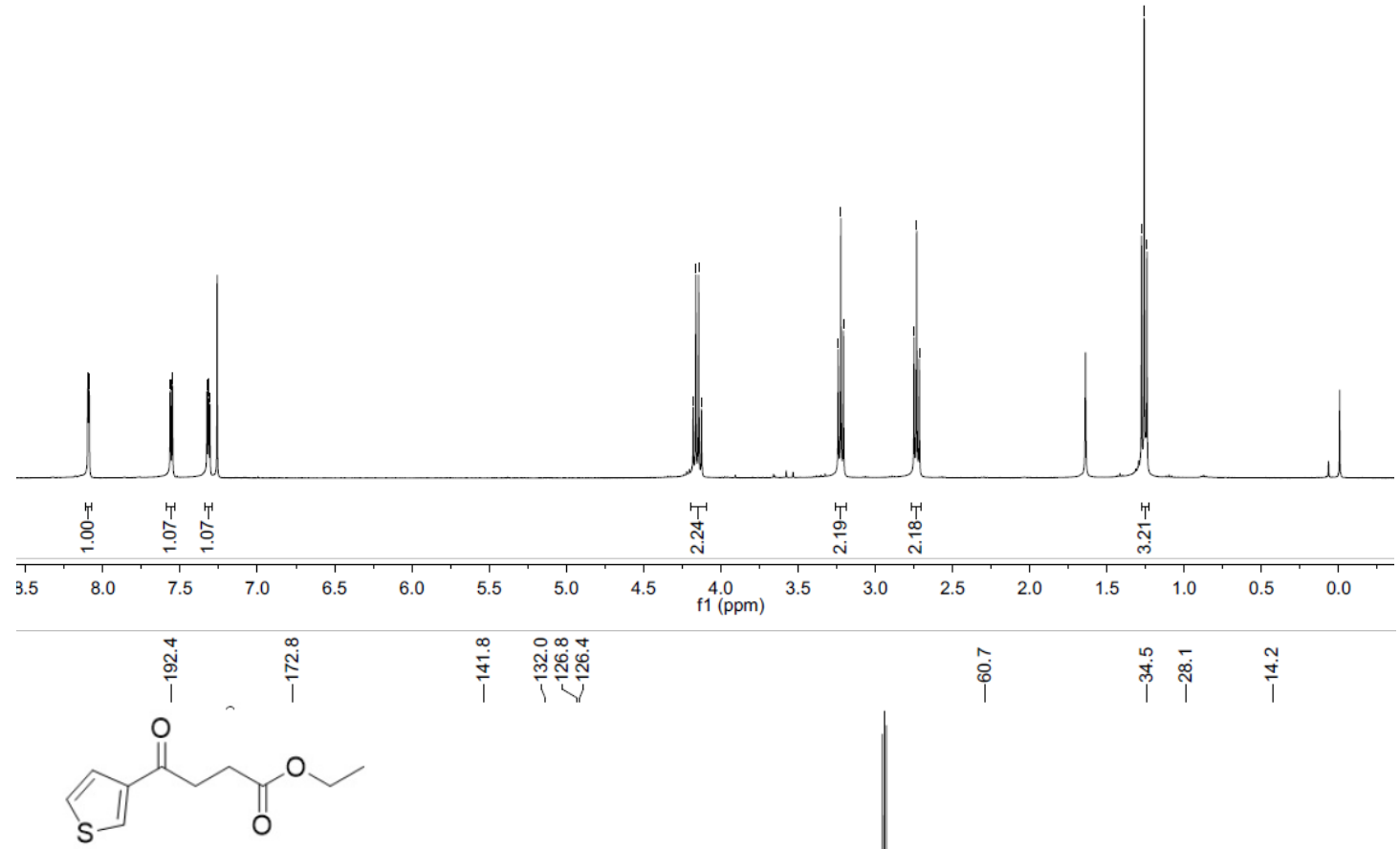

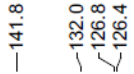

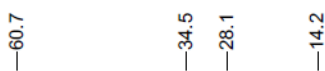

${ }^{13} \mathrm{C}$ NMR $\mathrm{CDCl}_{3}, 101 \mathrm{MHz}$

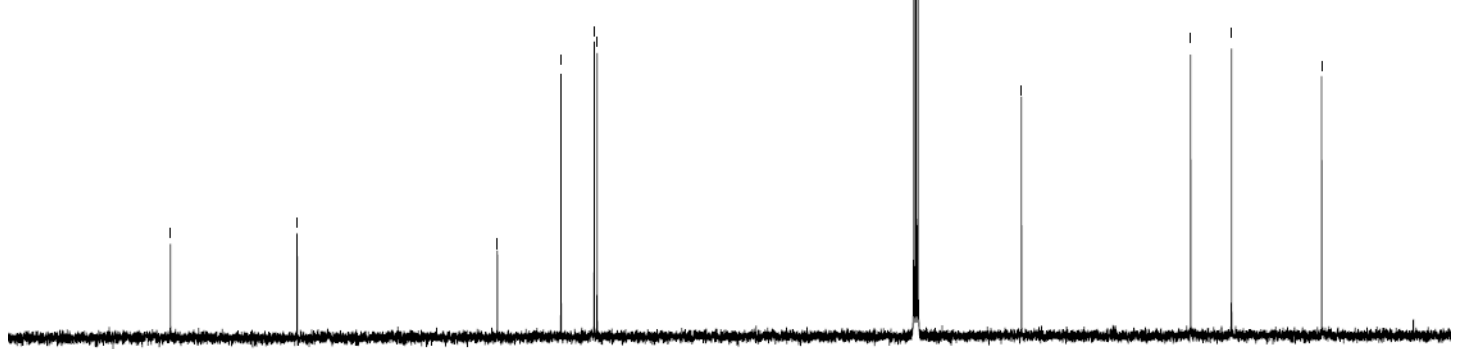

$\begin{array}{llllllllllllllllllllllllllllllll}210 & 200 & 190 & 180 & 170 & 160 & 150 & 140 & 130 & 120 & 110 & 100 & 90 & 80 & 70 & 60 & 50 & 40 & 30 & 20 & 10 & 0\end{array}$ 
3B:

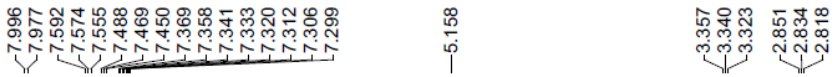<smiles>O=C(CCC(=O)c1ccccc1)OCc1ccccc1</smiles>

${ }^{1} \mathrm{H} \mathrm{NMR} \mathrm{CDCl} 3,400 \mathrm{MHz}$
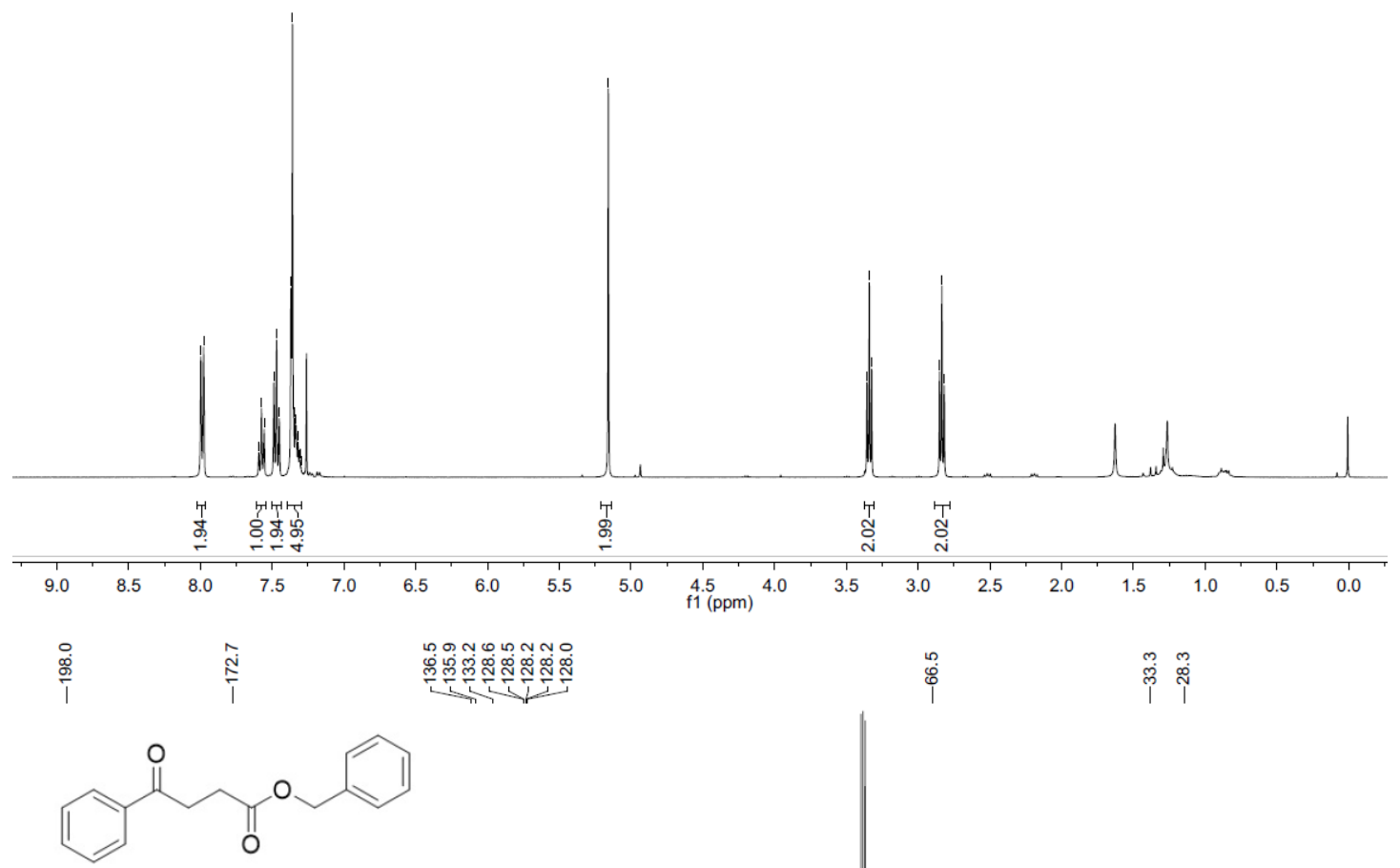

${ }^{13} \mathrm{C} \mathrm{NMR} \mathrm{CDCl}_{3}, 101 \mathrm{MHz}$

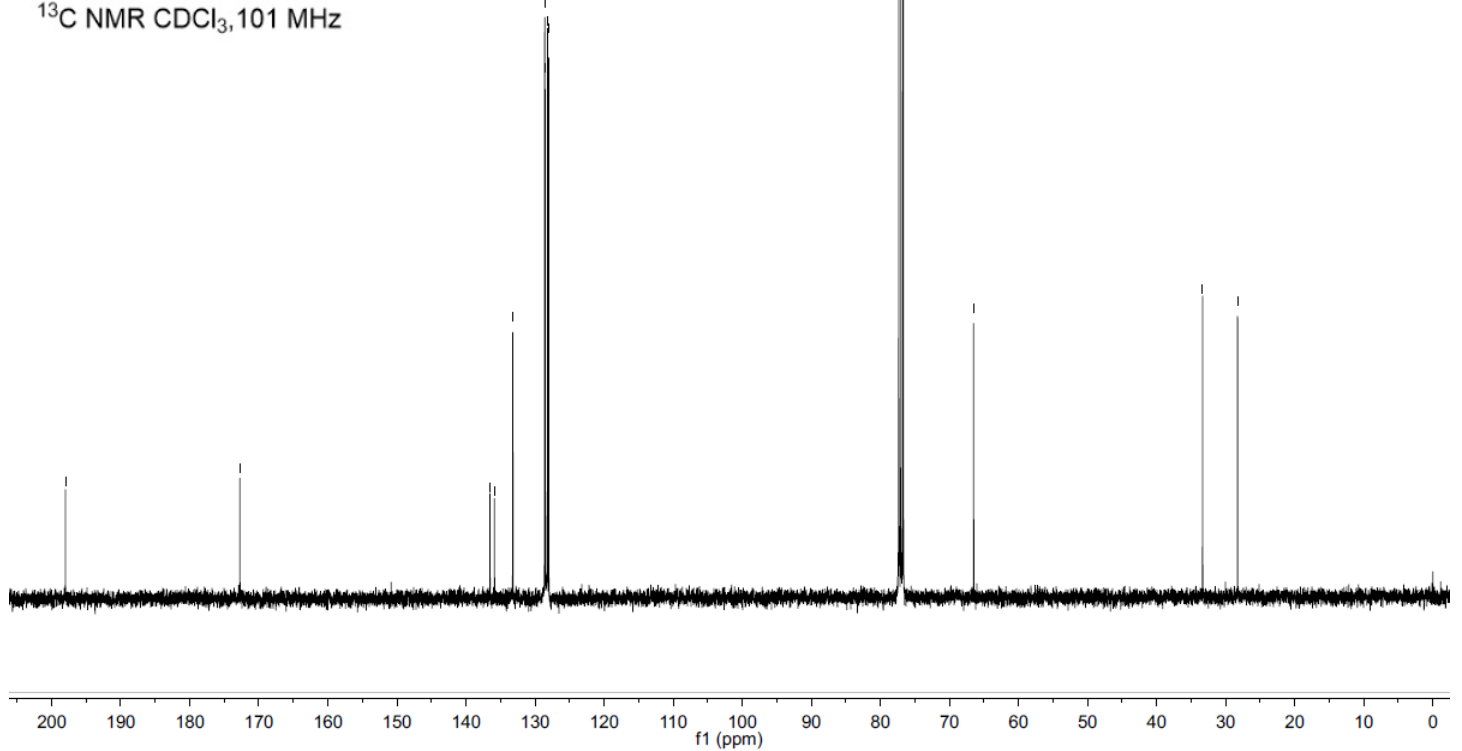


3C:

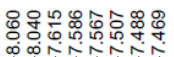<smiles>O=C(CCC(=O)c1ccccc1)c1ccccc1</smiles>

${ }^{1} \mathrm{H} \mathrm{NMR} \mathrm{CDCl}_{3}, 400 \mathrm{MHz}$

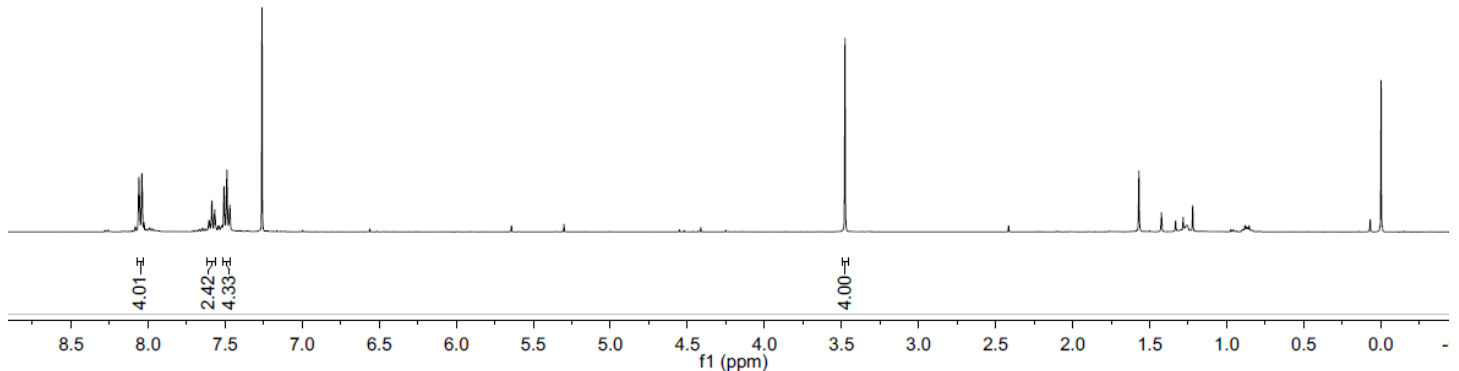

$\stackrel{\hat{\phi}}{\stackrel{\rho}{i}}$

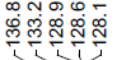<smiles>O=C(CCC(=O)c1ccccc1)c1ccccc1</smiles>

${ }^{13} \mathrm{C} \mathrm{NMR} \mathrm{CDCl}, 101 \mathrm{MHz}$

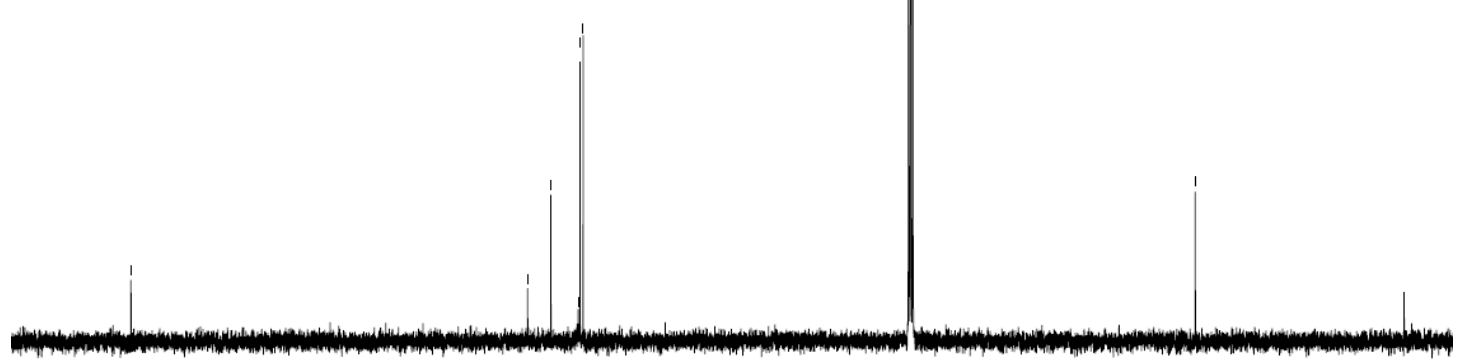

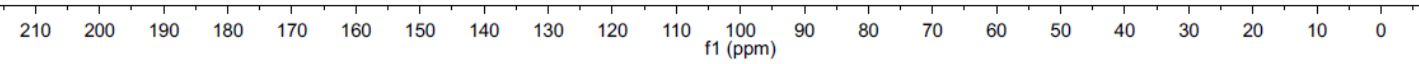


3D:

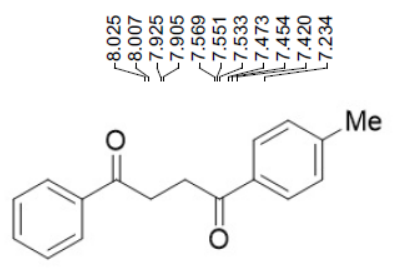

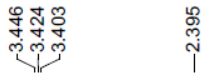

${ }^{1} \mathrm{H} \mathrm{NMR} \mathrm{CDCl}{ }_{3}, 400 \mathrm{MHz}$

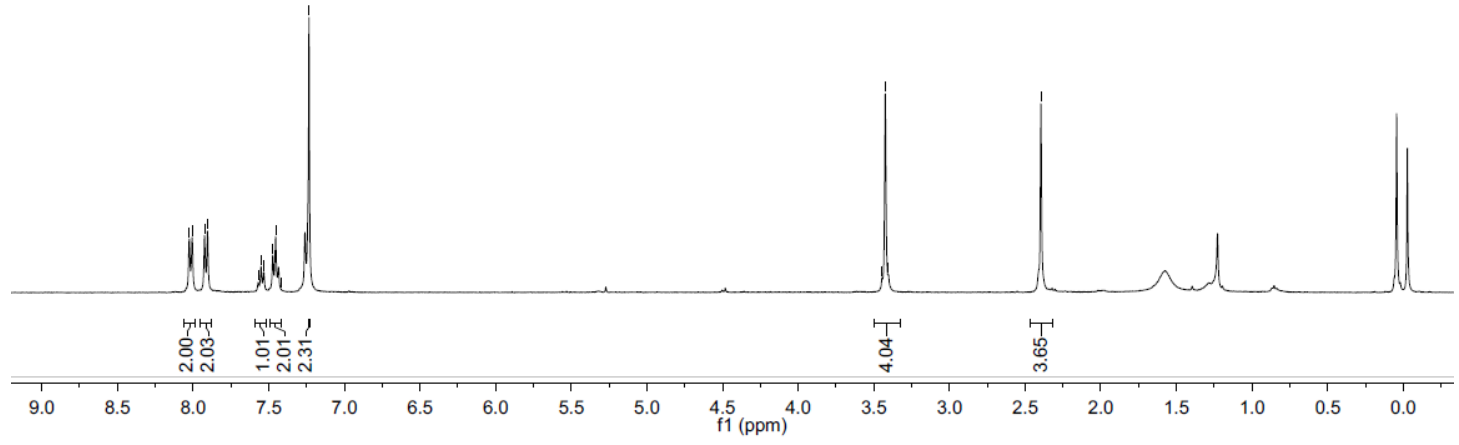

这

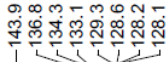

舟踏

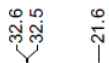

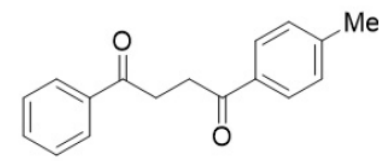

${ }^{13} \mathrm{C} \mathrm{NMR} \mathrm{CDCl}_{3}, 101 \mathrm{MHz}$

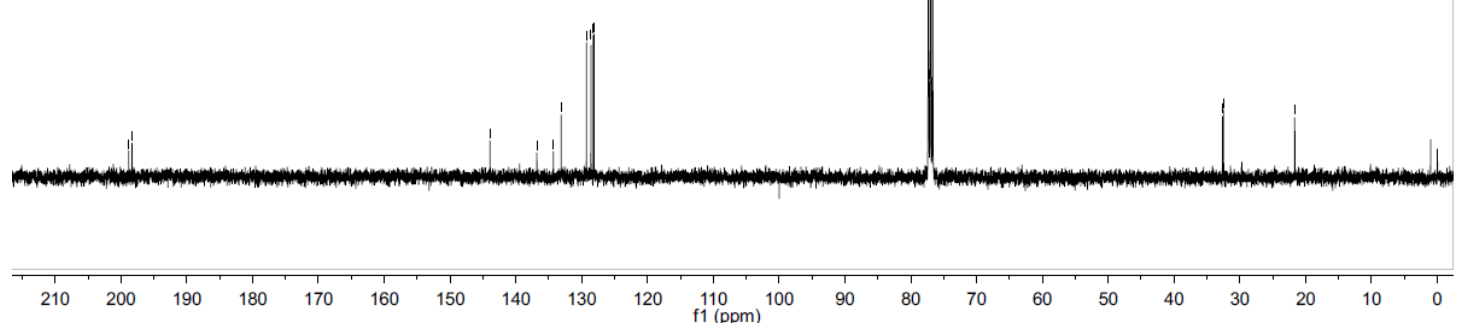


3E:

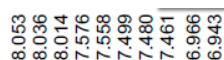

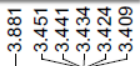<smiles>COc1ccc(C(=O)CCC(=O)c2ccccc2)cc1</smiles>

${ }^{1} \mathrm{H} \mathrm{NMR} \mathrm{CDCl} 3,400 \mathrm{MHz}$

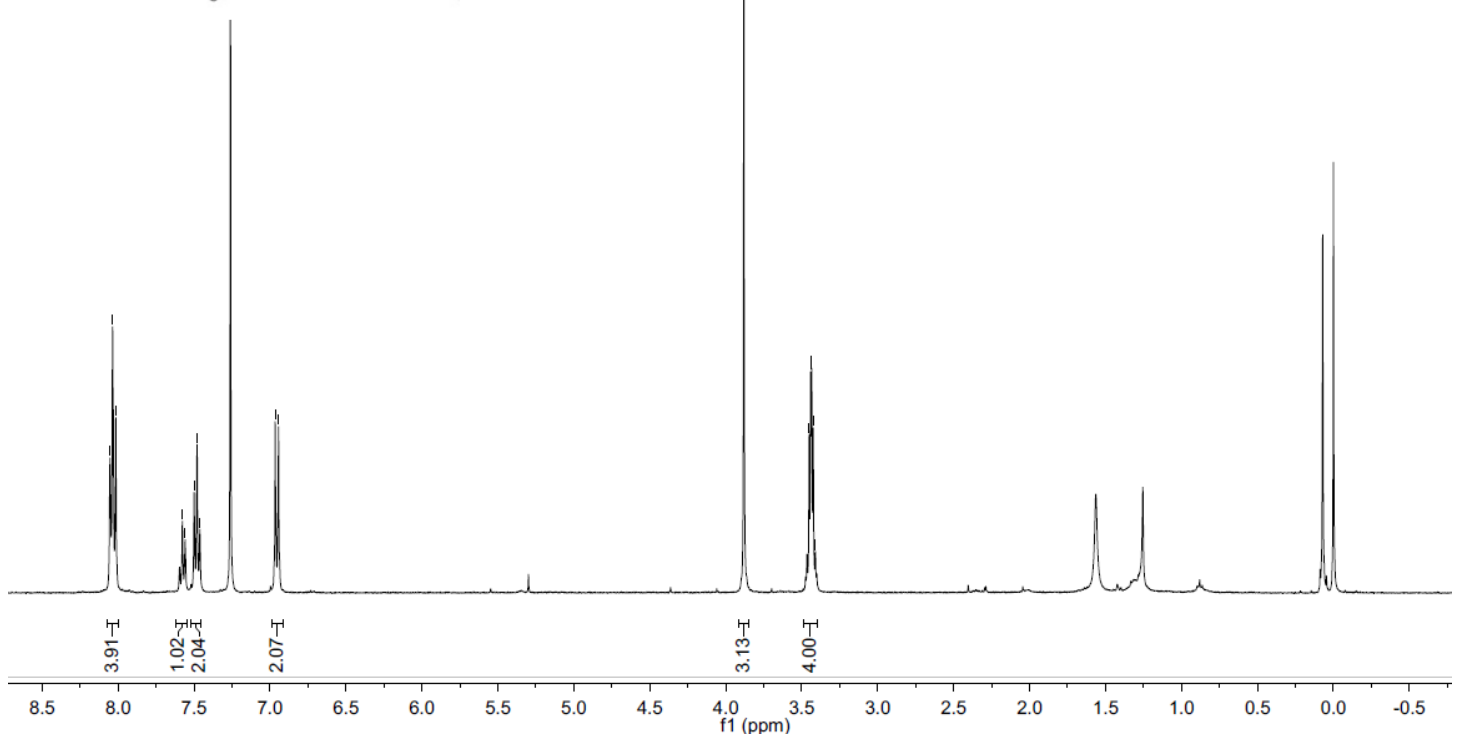

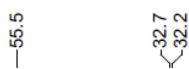<smiles>COc1ccc(C(=O)CCC(=O)c2ccccc2)cc1</smiles>

${ }^{13} \mathrm{C} \mathrm{NMR} \mathrm{CDCl} 3,101 \mathrm{MHz}$

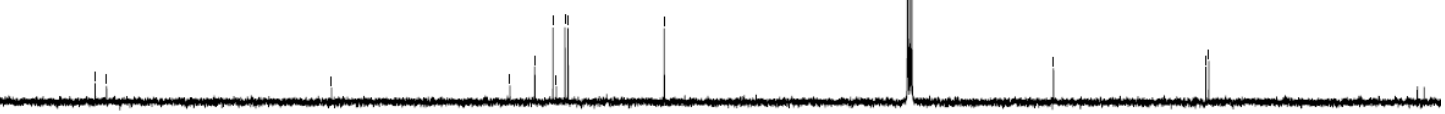

$\begin{array}{lllllllllll}210 & 200 & 190 & 180 & 170 & 160 & 150 & 140 & 130 & 120 & \begin{array}{c}110 \\ \mathrm{f} 1(\mathrm{ppm})\end{array}\end{array}$ 